EXPLORATION SGIENTIFIQUE DE LA TUNISIE.

\title{
PRODROME
}

\section{DE LA MALACOLOGIE}

TERRESTRE ET FLUVIATILE

DE

\section{LA TUNISIE,}

PAR

A. LETOURNEUX,

ทEMBRE DE LA MISSION DE L'EXPLORATION SCIENTIFIQUE DE LA TINISIE,

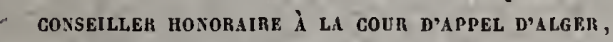

OFFIGIER DR LA LÉGION D'HONNEUR, ETG. ,

ET

\section{J.-R. BOURGUIGNAT,}

SECrétaloe géNéral de la société malagologrǫue de Fraxce,

- chevalier de la léglox d'hoxxeur, etc.

\section{PARIS.}

IMPRIMERIE NATIONALE.

M DGCG LXXXVII.

18.7 


Digitized by the Internet Archive in 2017 with funding from IMLS LG-70-15-0138-15 


\section{EXPLORATION SGIENTIFIQUE \\ DE LA TUNISIE, \\ PUBLIÉE}

SOUS LeS AUSPIGES dU Ministère DE L'INSTRUCTION PURLiQUe.

\section{ZOOLOGIE. - MALAGOLOGIE:}




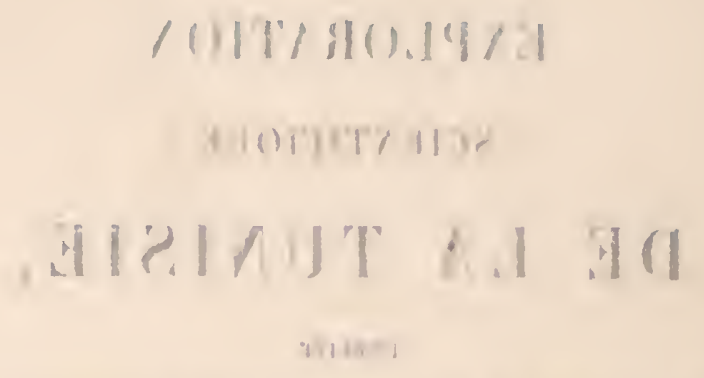

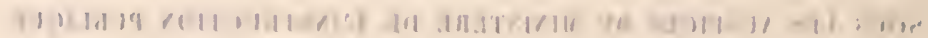

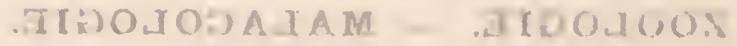


EXPLORATION SGIENTIFIQUE DE LA TUNISIE.

\section{PRODROME}

\section{DE LA MALACOLOGIE}

TERRESTRE ET FLUVIATILE

DE

\section{LA TUNISIE,}

PAR

A. LETOURNEUX,

MEMBRE DE LA MISSION DE L'EXPLORATION SGIENTIFIQUE DE LA TUNISIE, CONSEILLER gONORAIRE $\boldsymbol{\lambda}$ LA COUR D'APPEL D'ALGER, OFFICIER DE LA LÉGION D'HONNEUR, ETC.,

ET

\section{J.-R. BOURGUIGNAT,}

SECrétaire géxéral de la société MaLacologique de FRANCE, CHEVALIER DE LA LÉGION D'HONNEUR, ETC.

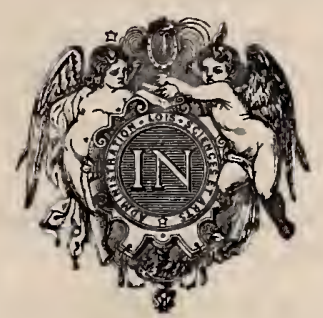

PARIS。

IMPRIMERIE NATIONALE.

M DCGC LXXXVII. 


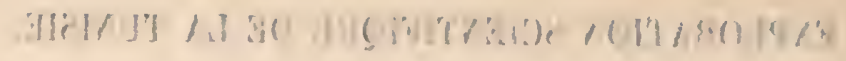

\section{ANOSOOHY}

\section{古员)}

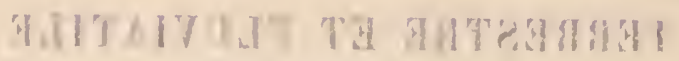

11
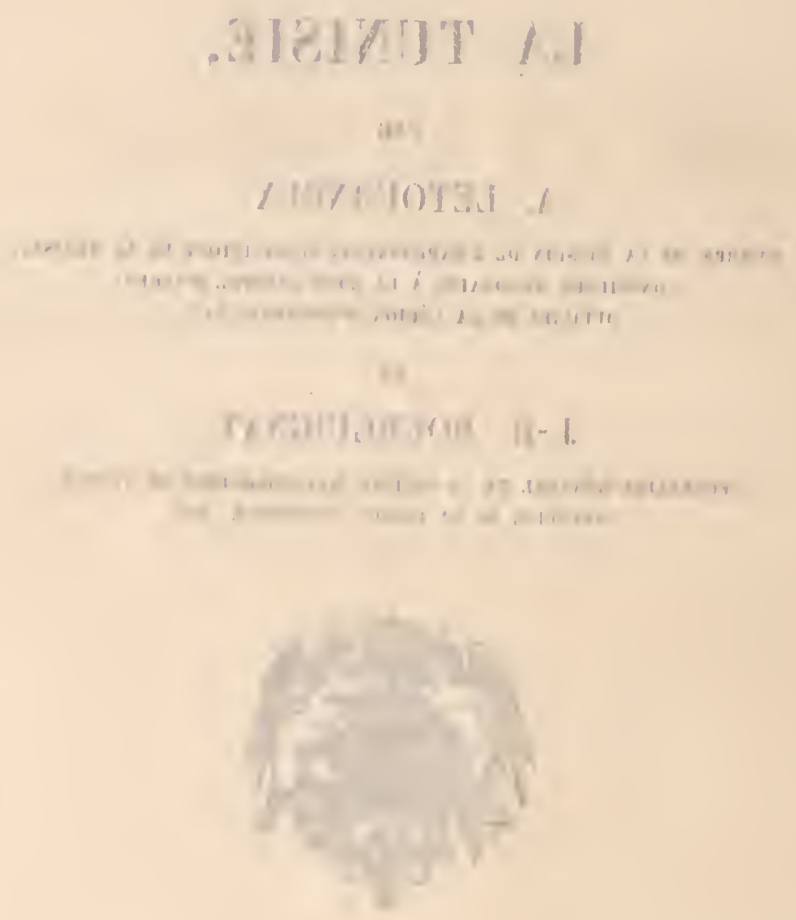

. 19199.

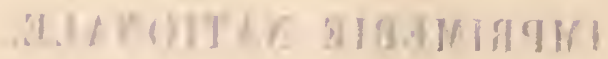

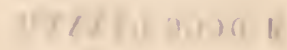




\title{
PRODROME
}

\section{DE LA MALACOLOGIE}

\author{
TERRESTRE ET FLUVIATILE
}

DE

\section{LA . TUNISIE.}

\section{GASTEROPODA INOPERCULATA.}

\author{
PULMONACEA.
}

LIIIA C ID $A$.

$\operatorname{MILAX}{ }^{(1)}$.

M. gagates Gray, Cat. of Puln. or air-breath. Moll. I, p. 174, 1855. (Limax gagates Draparnaud, Tabl. Moll. p. 100, 1801, et Hist. Moll. Fr. p. 122, pl. IX, f. 1, 1805.)

Sous les pierres, au pied du Djehel Abdellah près le Cap Roux; Ariana près Tunis (Bourg. 1867); sous les détritus au Djebel Reças (Lei.).

Ce Limacien, le seul connu jusqu“à présent dans la Régence, est une espèce cosmopolite, répandue dans toutes les régions circumméliterranéennes.

(1) Gray, Cat. of Pulmon. or air-breath. Moll. I, p. 162 et $17^{4}, 1855$, et Bourguignat, Malac. Alg., I, p. 45, 1864. - Limax s. g. Amalia Moquin-Tandon, Hist. Moll. Fr. II, p. 18, 1855. — Genie Amalia des auteurs allemands.

Une fois pour toutes, nous croyons utile d'établir la priorité de ce genre MıLıx, priorité contestée par les auteurs étrangers qui veulent l'accorder à l'appellation d'AmaLia.

L'Histoire naturelle des Mollusques de France, par M. Moquin-Tandon ( 2 vol. in- ${ }^{\circ}, 1855$ ), où se trouve inscrit pour la première fois le nom d'Amalı, a été publiée en six livraisons : la première date du 12 avril 1855 , la deuxième du 4 mai, la troisième du ¿ $^{\text {or }}$ aoùt, la quatrième du 10 septembre de la mème année; enfin la cinquième du a janvier, et la sixième et dernière du 9 avril 1856. La préface de cette Histoire, portant la date du 15 mars (date inexacte), n'a paru qu'au $1^{\mathrm{ex}}$ août avec la troisième livraison. L'ouvrage de Gray (Cat. of Pulmon. I, 1855, avec une préface en date du 29 mars) a été livré alı public savant le ${ }^{\text {er }}$ mai de la mème année; cet ouvrage est donc antérieur de quatre mois et demi à la quatrième livraison (10 septembre) de MoquinTandon, où se trouve établie la coupe s. g. Amsus. L'antériorité appartient, comme on le voit, incontestablement au genre Mrudx.

Malacologie. 


\section{HILICID $A$ E.}

\section{HYALINIA ${ }^{(1)}$.}

M. Pomeliana Issel, Moll. croc. Viol. in Mus. civ. Gen. 1880, p. 261. (Zonites PomeJianus Bourg. Moll. nouv. VIII, 1867, p. 229, pl. XXXVII, f. 17-20.)

Près d'une source sur la côte méridionale de l'île de la Galite (Issel).

\section{$\$ 1$.}

I1. psatura Locard, Prodr. malac. Fr. p. 39, 1882. (Zonites psaturus Bourg. Malac. Alg. I, p. $7^{4}$, pl. IV, f. $\left.30-33,1864.\right)$

Espèce rare. Un échantillon jeune sous les pierres au Cap Bon (Let.).

\section{\$2.}

I. cheliella Pechatd, Exc. malac. n. Afr. ( $1^{\text {er }}$ fasc. 1883$)$, p. 15 et 23 .

Ruines de Garthage (Bourg. 1867). Pen commune.

H. subplicatula Bourguignat, 1882, el in Pechaud, Exc. mal. n. Afr. p. 16. et a3, 1883. (Zonites subplicałulus Bourg. Mal. Alg. 1l, 1864, p. 3o4, et Mal. Tunis, p. 9, pl. I, f. 5-9, 1868.)

Sous les pierres à Carthage (Bourg. 1867), au Djebel Abd-er-Rhaman et dans les liantes vallées de la Medjerda (Let.).

\section{s 3 .}

II. Cossonii Letourneux et Bourguignat, sp. nov.

Testa pervie lateque umbilicata, depressa, supra vix convexa, fragili; subdiaphana, uniformiter pallide cornea et subtiliter striatula; anfractibus 6 conrexiusculis, arctatis, regulariter crescentibus, sutura impressa separalis; ultimo vix majore, compresso, obsolete in medio subangulato, supra convexiusculo, sublus ad initium leviter subplanulato, ad insertionem labri recto; aperlura parum obliqua, transverse lunato-oblonga, peristomate recto, acuto, fragili; alt. 4, diam. 9 millim.

Cette Hyalinie, à laquelle nous attribuons le nom du savant Président de la Mission de l'exploration scientifique de la Tunisie, M. le docteur Ernest Cosson, mernbre de l'Institut, a été recueillie morte, sous des détritus, dans le Djebel Zaghouan, où elle paraît fort rare. - L'H. Cossonii appartient à la série des $H$. Magonensis, Metria et Gymnesiaca (Bourg.) des illes Baléares. Elle est remarquable par sa forme déprimée, ses tours serrés, et notamment par son large ombilic en entonnoir, qui laisse voir en entier l'enroulement spiral interne.

(1) Agassiz, 1837 (non Hyalina Schunaker 1817 et Studer 1820). Lès eapèces de ce genre avaient toutes été comprises autrefois dans le genre Zosites, genre spécialement réservé actuellement pour les formes de la série de l'H. Algirus. 
$\S 4$.

I. perspectivala Bourg. in Pechaud, Exc. mal. 11. Afr. 1883 , p. 23 . (Zouites perspectivulus $H$. Blanc, ms. 1879.)

Espèce bien caractérisée. Ruines de Carthage, où elle a été importée du sud de l'Italie.

\section{\& 5 .}

Ir. eustilba Bourguignat, $\mathbf{1 8 8 2}$, in Pechaud, Exc. mal. n. Afr. 1883, p. 24 . (Zonites Eustilbus Bourg. Mal. Alg. I, 1864, p. 76 , pl. IV, f. 11-16, et Mal. Tunis, p. 9, 1868.)

Ruines d'Utique, sous les pierres (Bourg. 1867).

I. Subvitreola Boung. in Pechaud, Exc. mal. n. Afr. 1883 , p. 17 et 24 . (Zonites subvitreolus Bourg. in Servain, Moll. Esp. p. 28, 1880.)

Alluvions de la Medjerda, près de son embouchure dans la lagune de PortoFarina, et ruines d'Oudena au sud de Tunis (Bourg. 1867).

II. diauga Bourg. in Pechaud, Exc. mal. 11. Afr. p. 17 et 25,1883 . (Zonites Diaugrts Bourg. cité par Servain, Moll. Esp. p. $28,1880$.

Environs de la gare de Ghardimaou (Pechaud, 1882).

Espèce rare, ou plutôt difficile à trouver, ainsi que les deux précédentes, à cause de son extrême exiguité.

\section{LEUCOCHROA ${ }^{(1)}$.}

L. Otthiana Albers, Heliceen, p. 79, 1860. (Helix Otthiana Forbes, in Ann. of Nat. Hist. p. 282, 1838, et supplém. tab. XI, f. 2, 1839. - Helix Jeannotiana Terver, Cat. Moil. Alg. p. 20, pl. II, f. 11-12, 1839. - Zonites Otthianus Bourg. in Amén. Nalac. II, p. 153,1859 , et Malac. Alg. I, 1864, p. 79 , pl. VI, f. 6-1 o, et Malac. Tunis, p. 10, 1868.)

Sur les rochers au cap Roux.

I. cendidissima Beck, Ind. Moll. p. 17, 1837. (Helix candidissima Drapartaild, Tabl. Moll. p. 75,1801 , et Hist. Moll. Fr. p. 8 , pl. V, f. 18, 1805. - Zonites candidissimus Moquin-Tandon, Observ. mach. Hél. in Mém. Acad. Toulouse, $3^{\mathrm{c}}$ série, IV, p. 374,1848 .)

Excessivement abondante dans toute la Régence; cette espèce est même si répandue qu'il est inutile de citer les nombreuses localités où elle a été constatée.

Ses principales variétés sont :

VıR, maxima Bou'g. Mal. Alg. I, p. 87, 1864.

Ruines d'Oudena (Bourg. 1867); Haguet-Hammor près le Cap Bon (Bellucci, ${ }_{1875}$ ); collines entre Sousa et El-Djem (Gestro, $18_{7} 6$ ).

V.ı. minima Bourg. loc. cit. p. 87 .

Collines du Cap Bon (Gestro, 1876).

(1) Beck, 1837. Autrefois les espèces de ce genre étaient classées soit parmi les Helix, solt parmi les Zonites. 
VAr. conoidea Bourg. loc. cit. p. 87 .

Haguet-Hammor, au Cap Bon; mont Mida; mont Tebagua (Bell. 1875); Djebel Reças; monlagne des Malmata, Ghomrasen et Zarzis (Lel.).

VAr. subcarinata Bourg. loc. cit. p. 87. (Helix rimosa Jan, in Rossm. Iconogr. Vl, 1837 , f. $367 \cdot$ )

Djebel Reças; région des Hamada (Let.).

Van. depressa Bourg. loc. cil. p. 87. (Helix depressa Muhlferldt.)

Cap Bon; boı'ds de l'Oued Cherb el de rOued Taferma (Let.).

Yis. obtuso-producta.

Foum-el-Bouibet (Let.).

Vir. umbilicata Bourg. loc. cit. p. 87. (Helix candidissina var. umbilicata Menke, Syn. Noll. p. 16, 1831. - Leucochroa candidissima var. perforata Morlet, Coq. exp. Cholts, in . Irch. miss. sc. 3 " série, Vll, 1881, p. 393.$)$

Plaine d'Hanıëmet (André. 1878).

La L. candilissima est une espèce cosmopolite répandue dans toutes les régions núdilcranécnnes soumises aux influences marines.

1. Bactiea Albers, Ileliceen, p. 79, 1860. (Helix Bretica Rossmässler, in Zeilsch. f. Malak. p. 170,1853 , et lconogr. XIII et XIV, 1854 , p. 16 , f. 819-813. - Zonites Bieticus Bourgro. in Amén. malac. II, p. 15. 1859.)

Cette espèce espagnole a été constatée par M. Morlet (Arch. miss. sc. 1881, p. $\left.3 g^{3}\right)$ au sud de la 'Tunisie, dans la plaine d'Haneïmet.

Vin. Tunetana (L. Tumelana Letourneux et Bourguignat).

Sur les rochers de Guelaat-es-Snam (let.).

Cetle forme, qui mérite d'être distinguée sous un nom spécial, bien que nous lúsitions pour le moment, à cause de sa rarelé, à l'élever au rang spécifique, ressemble an type de Nurcie, tont en s'en dislinguant par son test finement strié en dessus, non chagriné, par son bord péristoual simple, très Iranchant, non obtus-épaissi, et par le filet carénaut de son dernier tour à peine accentué.

\section{HELIX ${ }^{(1)}$.}

Les espèces du genre Ilelix sont largement représentées dans la Régence, surtout cello's qui appartiemuent aux séries xérophiliennes. Nous ne connaissons pas dans le système emropéen, à l'exception de quelques régions algériennes, de pays oì elles soient anssi magnifiquement développées. Les contrées tunisiennes sont pour elles ce que sont les péniusules italique el turco-hellénique pour les séries campyléennes, ce que sout les monlagnes daluales pour les Clausilies. Nous nous attacherons d'une façon toute particulière à donner une connaissance parfaite des

(I) Liunaus, 1758 . 
Hélices xérophiliennes caracléristiques de cette faune, parce qu'elles méritent d'être mises en lumière.

Dans les différents mémoires publiés sur la Tunisie, on rencontre un certain nombre d'Hélices inscrites sous les noms d'H. lauta, lineata, variabilis, neglecta, subrostrata, etc., dont nous ue parlerons point, parce que, sous ces appellations, 'videmment fautives, se cachent des formes tout autres que celles dont elles portent les noms.

Nous passerons également sous silence les Helix soluta et chilembia signalées par M. Morlet (in Arch. miss. sc. 1881, p. 3,4) dans le sud de la Régence, parce que ces deux espèces sont des formes incontestablement mal déterminées.

\section{$\S 1$.}

I. aperta Born. Mus. Vindob. p. 387 , tab. XV, f. 19-20, 1780 .

Dans les champs, au pied des Olivier's, des Opuntia, etc. Environs de Tunis, à la Manouba, au marabont Sidi-Fellala, à l'Ariana, à Kannart près le palais en ruine de l'ancien ministre Ben-Hayet; rersants du Djebel Ahmar (Bouro. 1867); Hammam-el-Lif, oasis de Gabès (Bell. 1875); entre Tunis et Utique (Gestro, $187^{6}$ ); Tebourba (André, 1878 ).

II. Kalaritana Prunner, mss. teste Villa, Conch. isola di Sardegna, p. 1, 1836, Disp. system. Conch., p. 10, 1841. (Helix aperta (pars) Issel, Moll. croc. Vio. in Nus. civ. Gen. $188_{2}$, p. 266 .)

Le docteur Issel mentionne un individu de l'H. aperta de petite dimension, spire relativement élevée, à ouverture extérieurement peu arrondie et au dernier tour orné d'une ride longitudinale assez prononcée. Ces signes convienuent à l' $H$. Kularitanı, plus petite que l'H. aperta, qui a une spire élevée, une ouverture moins arrondie, plus allongée dans le sens vertical et qui souvent offre sur la partie supérieure du dernier tour une ride longitudinale plus ou moins accentuée.

L'H. Kalaritana vit en Sardaigne, en Sicile, en Grète et dans le sud de l'Italie. D’après le docteur Issel, cette forme existerait en Tunisie aux environs d'Utique.

II. aspersa Müller, Verm. Hist. II, p. $59,177^{4}$.

Espèce très abondante dans tous les environs de Tunis, aux ruines d'Utique, de Carthage, d'Oudena, etc.; dans la vallée de la Merjerda à un kilomètre du Fondouk, où l'on en trouve de magnifiques échantillons.

A Aïn-Draham et à Fedj-Saha en Kroumirie, on rencontre des individus de l'H. aspersa presqiie semblables comme coloration, comme striation, comme élévation spirale, à l'H. Mazzulii de Sicile. Dans une couche de travertin de l'ile de la Galite, le docteur Issel a trouvé également une $H$. aspersa très voisine de l'espèce sicilienne.

\section{$\$ 2$.}

I. pachya Bourg. in Amén. Malac. II, p. 180, pl. XXI, f. 6-9, 1860, et Malac. Alg. I , p. 100 , pl. VII. l'. $5-8,1864$.

Échantillons bien typiques à Houmt-Souk dans l'iłle de Djerba (Let.), et çà et là sur le littoral. Hélice importée de Syrie. 
I. melanostoma Draparnaud, Tabl. Moll. p. $7^{8,1801}$, et Hist. Moll. Fr. p. 91, pl. V, f. 24,1805 , et Bourg. Malac. Alg. I, 1864, p. 96, pl. VII, f. 11-13.

Environs de Tumis et vallée de la Medjerda, oǹ elle est très abondante (Bourg. 1867); Hamman-el-Lif et collines près du Cap Bon (Bell. 1575): Tebourba,

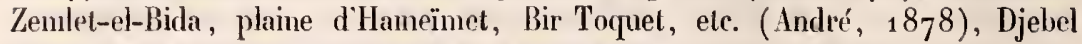
Reças, bois de Pins près de Haïdra, Aïn Bonderiès, Gafsa et Tozer (Let.).

V.n. maxima Bourg. Mal. Ag. I, p. $9^{8}, 1864$.

Échantillous de 5 o à 53 mill. de hauteur sur 42 à 44 de diamètre, près d'un vieux puits espagnol à un kilomètre au sud-est de Tunis (Bourg. 1867), Carthage (Bell. 1875); entre Sousa et El-Djem (Gestro, 1876).

H. melanonixia Bourg., Test. noviss. $n^{\circ} 71,1876$.

Cette Hélice algérieune se trouve bien caractérisée à Hount-Souk dans l’île de Djerba (Let.).

I. Ciecensis Bourg. in Pechaud, Exc. malac. n. Afr. 1883, p. 35. (Ifelix melanostoma var. conoidea Issel, Moll. croc. Viol. in Ann. mus. civ. Gen. 1880, p. 365.$)$

Ruines dUtique (Bourg. 1867); groltes d'El-Haouiria près le Cap Bon (Bell. ${ }_{1875}$ ); collines nutre Sousa et Bir El-Buita (Gestro, 1876 ).

II. nueula Purreyss in L. Pfeiffer, Mon. Hel. viv. IV, p. 161,1859 , et Bourg. Malac. Mg. I, 1864 , p. $9^{8}$, pl. VlI, f. 9-10.

Euvirons de Tunis, ̀̀ Kamart el à Porto-Farina (Bourg. 1867); Kriz (André, 1878); iles de Kerkemna (Douumet-Arlanson, 1884).

Espèce importée de l'Égypte. Les échantillons hmisiens sont identiquement semblables ì ceux des environs d'Alexandrie.

\section{$\$ 3$.}

H. vermiculata Miiller, Verm. Hist. II, p. 20, $177^{4}$.

Hélice des plus communes aux alentour's de Tunis, sur les versants du Djebel Alurur, dans la vallée de l'Oued Meliana, et aux environs de Porto-Farina (Bourg. ${ }_{1867}$ ); mont Haguet-Hammor, mont Mida, mont Tebagua, Djebel Reças, Monastir, oasis de Mluja (Bell. 1875); collines entre Sousa et El-Djem (Gestro, 1876); La Galite (Issel, 1877); Oued Akarit, plaine d'Hameïnel, Zcmlet-el-Bida, Bir Toquet, etc. (André, 1878); îles de Kerkenna (Doúmet-Idanson, 1884 ); ile Tabarka, Matnıta, Ketenna, Sebkha Zerguin, Gabès, Gafsa, Hount-Souk daus l’ìle de Djerba (Let.).

V.A. minuta.

Cartlage (Bell. 1875 ); iles de la Galite, Galitone, Eguglia et Gallina (Gestro, ${ }_{187} 6$ el lssel, ${ }_{1877}$ ); El-Bida (André, ${ }_{1878}$ ).

Vin. pelagosana (Helix Pelagosa, Stossich).

İles de Kerkenna (Doûmel-Arlanson, 1884). 
H. acouria Letourneux et Bourguignat, sp. nov.

Testa imperforata, depressa, nihilominus sat globosa, supra subtectiformi-convexa, subtus a sinistra ad dextram oblique convexa, opacula, striatula, in ultimo passim quasi crispulata, lutescente, zonulis 4 rufis (superioribus 2 latis, sæpe confluentibus, punctulis albidis varie dispositis ornatis; inferioribus 2 angustis, non interruptis) circumcincta; spira leviter convexa, in tholo subtectiformi-gibboso; apice lævigato; anfractibus 5 subconvexiusculis, celeriter crescentibus, sutura parum impressa separatis; ultimo maximo, amplo, ad initium subangulato, ad aperturam subrotundato, ad insertionem labri descendente; apertura obliqua, lunata, transverse oblique semiovata, peristomate albo, labiato, undique expanso ac reflexo; margine basali stricto, recte inclinato, in medio subconvexo; marginibus sat remolis, callo translucido junctis; alt. 17, diam. 27 millim.

Environs d'Houmt-Souk dans l'île de Djerba (Let.).

On rencontre près de Porto-Farina une variété un tant soit peu plus globuleuse, dont le dernier tour n'est pas subanguleux à l'origine.

Cette Hélice, remarquable par son test globudeux-déprimé, offrant en dessus une spire subtectiforme-arrondie, et, en dessous, une convexité sensiblement portée du côté de l'ouverture, par sa croissance spirale très rapide, par sa suture presque superficielle, par son dernier tour relativement très développé, subanguleux à l'origine et médiocrement descendant à linsertion du labre, parait voisine par son mode de coloration de l'H. vermiculata, avec laquelle, néanmoins, il n'est pas possible de la confondre.

H. Constantinse Forbes, Land and freshw. Moll. of Alg. in Ann. nat. Hist. or Mag. p. 251,1838 , et supplém. pl. XI, f. 1, ${ }_{1839}$. (Hetix Cirtæ Terver, Moll. n. Afr. p. 11, pl. I, f. 1, 1839).

Espèce très répandue aux environs de Tunis, notamment dans la direction de Mahamedia et des ruines d'Oudena (Bourg. ${ }^{1867}$ ). Elle a été encore recueillie à l'île de Tabarka (Let.), à Tebourba (André, 1878), à Ghardimaou (Pechaud), à Bizerte (Berthier), ainsi que dans le Djebel Reças et les contrées entre El-Djem, Kerouan et El-Kef, non moins quà Feriana, aux ruines d'Haïdra et"sur les pentes du Guelaat-es-Snam (Let.).

Ses principales variétés sont :

VAR, conoidea (Haut. 21, diam. 22 mill.).

Sur les collines de Sidi-ben-Hassen près de Tunis (Bourg. 1867) et à Ghardimaou (Let., Pechaud).

VAR. minima (Haut. 16, diam. 20 mill.).

Très abondante aux portes de Tunis, près du marabout Mahoubia; ruines d'Utique (Bourg. 1867); bords du golfe de Tunis (Gestro, 1876). 
H. Fleurati Bourg. Nalac. Tunis, p. 12, pl. I, f. 1-3, 1868.

Excessivement commune aux alentours de Tunis dans les terres cultivées et dans les friches. Cliamps au sud et au sud-est de Tunis, entre un vieux puits espagnol, les collines de Sidj-ben-Hassen et la forteresse Bordj-el-Rais; ruines d'Oudena, d'Utique et də Carthage (B urg. 1867); environs d'El-Djem (Gestro, 1876 ); Tebourba (André, 1878 ); Ghardimaou (Pechaud).

VAr. subcariaata Bourg. Mal. Tunis, p. 13 , pl. I, f. 4.

Euvirous de Tunis.

Var. obesa Bourg. loc. cit. p. 13.

Coqnille petite, aussi haute que large.

Envirous de Tumis.

H. Bonduelliana Bourg. Mal. Alg. I, 1864, p. 116, pl. IX, f. 15-18.

Environs de la gare de Ghardimaou (Pechand, 1883).

1. Toukriana Bourg. Test. noviss, $\mathrm{n}^{\circ} 101,1876$, et in Pechaud, Exc. mal. n. Afr. I, 1883 , p. 37 .

Environs de la gare de Ghardinaou (Pechaud, 1883 ).

\section{S 4 .}

I. Pnniea Morelet, in Journ. Concl. II, 1851 , p. 35. , pl. IX, C. 3-4, et IV, 1853 , p. 287 ; et Bourg. Valac. Alg. 1, 1864 , p. 110 , pl. IX, f. $10-14$.

Cette belle esjèce, spéciale, en Algérie, aux hautes régions montueuses de la province de Constantine, n’a été constatíe en Tunisie que dans les monlagnes voisiures de la frontière algúrienne, sur les pentes du rocher de Guelaat-es-Snan (Let).

1. nitefacta Bourg. spec. noviss. $n^{\circ} 121,1876$, et in Pechaud, Exc. mal. n. Af. I, 1883 , p. 99 .

Entre Aïn-Tamagra et Feriana (Let.).

\section{S 5 .}

11. Rusicadensis Letournenx, in Ann. malac. I, $187^{\circ}$, p. $29^{8}$, et Malac. Kabylie in La Kahylie el les coulumes kalyyles, I, 1872 , p. 122.

Hélice peu commune : Djebel Zaghonan et Djebel Abd-er-Rahman (Let.).

מ. Chefiana Burguignat, in sthed. 1875 .

Tesla perangruste perforala, globoso-subeonnidea, supra conoidąli, sinblus convexo-rotunda a, obscure subangulata, fragili, subprellucida, unilormiter cornea, subilissime striatula ac sub lente acriore argulissine subnal'eata; spira subconica, sat elala; apice exiguo; anfractibus 6 convexiu culis, resulariter ac s t lente crescen ibus, sulura impressula separatis; "ltimo prarum majore, ad initium subaugulato (angul, pallida), ad apertu"am subrotundato, suprene ad insertionem labri regulariter raldeque descendente ac deflexo; apertura obliqua, lunata, semiro undata, 
intus leviter pallide corneo-subrosacea; peristomate recto, acuto, profunde sublabialo; margine columellari superne in triangulam formam late reflexo ac leviter perforationem obtegente; alt. 10 , diaın. 12-13 millim.

Cette Hélice découverte d'abord dans la plaine de la Cheffia au sud-ouest de la Calle, à l'est du. Djebel Bou-Abed (province de Constantine), a été retrouvée sous les détritus dans les Djebel Zaghouan et Bou-Kourneïn (Let.).

L'H. Cheffiana se distingue de l'H. Rusicadensis, la seule qui, parmi les nombreuses formes de cette série, peut lui être assimilée, par sa croissance spirale moins accélérée, par son dernier tour moins développé, plus exactement rond, moins subanguleux, offrant, à l'iusertion, une direction descendante plus longue et plus accentuée, par sa perforation encore plus étroite, par son ouverture plus exactement semi-sphérique; enfin, par sa forme plus conique, puisqu'elle atteint 10 millim. de hauteur, tout en n'ayant que 12 à 13 millim. de diamètre, tandis que celle de l'H. Rusicadensis en a 11 sur 16 ; aussi cette Hélice, par suite de son plus grand diamètre, paraitt-elle plus déprimée.

H. acorta Letourneux et Bourguignat, sp. nov.

Testa anguste perforata, depressa, supra mediocriter convexa, subtus convexiore (convexitate ad dextram validius expressa), sat valide angulata (angulo pallide subalbido, ad aperturam obsoletissimo), subpellucida, non nitente, uniformiter cornea ac sat striatula; spira subconvexa, tectiformi-subrotundata; anfraclibus 5 supra vix convexiusculis, celeriler crescentibus, sutura impressula separatis; ullimo majore, angulato, supra mediocriter convexo, subtus perconvexo præserlim ad aperturam, ad inilium compresso-angulato, superne ad inserlionem breviler subdescendente; apertura parum obliqua, ampla, lunata, semirotundata, intus pallide rosacea; peristomate recto, acuto, intus leviter profunde incrassatulo, inferne subpatulo, ad marginem columellarem late dilatato ac circa perforalionem sat circumambiente; alt. 8, diam. 13 millim.

Cette nouvelle forme, recueillie dans le Djebel Reças et sur une montagne de grès dans la haute vallée de la Medjerda (Let.), diffère de la précédente par sa coquille d’́primée, très convexe en dessous au niveau de l'onverture et à peine convexe en dessus en forme de dòme; par son dernier tour fortement anguleuxcomprimé à son origine et allant ensuite en augmentant en taille et en grosseur jusqu’à l'ouverture, qui devient, par cela mème, très développée; par sa croissance spirale plus rapide; par sa très courte descente plus accentuée à l'insertion du bord supérieur externe, etc.

\section{s 6.}

14. rosen-tineta Forbes, Moll. of. Alg. in Ann. nat. Hist. or Mag. p. 252, 1838 , et Supplém. pl. XI, f. 3 (mala), 1839, et Bourg. Malac. Alg. I, 1864, p. 154, pl. XVII, f. 8-12.

Sous les pierres aux ruines d'Oudena (Bourg. 1867); environs d'Utique et île de la Galite (Gestro, 1876 ). 
H. lanuginosa Boissy in Guerin, Mag. zool. pl. LXIX, 1835, et Bourg. Mal. Alg. I, 1864, p. 152 , pl. XVII, f. $1 \div 7$.

Sous les détritus au Djebel Reças (Let.).

\section{ऽ 7 .}

II. mendicaria L. Pfeiffer, Descr. Land Shells in Proceed. zool. Soc. London, p. 136, 1860.

Montagnes du centre de la Tunisie (Tristam).

$\$ 8$.

H. sordnlentat Morelet, in Journ. Coneh، 1851, p. 356, pl. IX, f. $9-10$, et Bourg. Mal. Ilg. I, 1864 , p. 187 , pl. XVIll, f. 17-20 (Exel. fig. 21).

Meızel-lou-Zelfa, et pentes de Guelaat-es-Snam (Let.).

I. slivehra Letourneux et Bourguignat, sp. nov.

Testa profunde angusleque perforata, globoso-depressa, supra convexa, subtus comvexiore, parum pellucida, non nitente, pallide cornea, striatula, sub lente acriore passim pilis brevissimis rare sparsis hirsuta; spira lepressa, parum producla; anfraclibus 5 convexiusculis, celeriter crescentibus, sutura.impressa separatis; ultimo majore, amplo, ad initium obscure subangulato atque ad aperturam paulalim ralide lumescente, superne ad iusertionem recio; apertura leviler obliqua, ampla, parmm lunala, fere circulari; peristonate recto, acuto, modo ad marginem columellarem dilatato ac palulo; alt. 5 , diam. 7 millim.

Rochers de Guclaat-es-Snam (Let.). Espèce peu conmune.

Celle Hélice diflère de l'H. sordulenta par sa perforation plus étroite, par sa spire moins élevée, par sa croissance spirale plus accélérée, par son ouverture plus anple, mais surtout par son dernier tour fort peu volumineux à l'origine et prenant pen à peu en arrivant vers l'ouschur nu développement relativement considérable. Dans ce tour, lígèrenent subanguleux à son commencement, le maximum de la convexité se trouve plus supérieur que celui de l'H. sordulenta, ce qui donne à ce tout un aspect bien différent.

I. chuoodia Bourg. Malac. Alg. I, 1864, p. 188, pl. XVIII, f. 26-29.

Sous les pierres daus les montagnes du Kef El-Djerdja (Let.).

II. persordida Letourneux et Bourguignat, sp. nov.

Testa aperte perforata (perforatione profunda, leviler pervia), depressa, xque convexa supra ac subtus, castanea, striatula, pilis brevibus hirsuta ac semper undique squalore obsita ac persordida; spira leviter subrolundato-convexa; apice læevigato; anfractibus 5 convexis, regulariter crescentibus, sutura angusta, profunda quasi canaliculata, separatis; ultimo majore, ad initium compresso, ad aperturam lumidiore, superne sensim 
leviter descendente; apertura parum obliqua, vix lunata, fere sphærica; peristomate recto, acuto, modo ad marginem columellarem superne leviter subdilatato; marginibus valde approximatis, fere junctis; alt. 4, diam. 7 millim.

Sous les détritus au Djebel Reças (Let.).

Cette espèce se distingue de la précédente par sa perforation moins ouverte; par ses tours de forme plus obèse, plus gros, tout en étant moins exactement ronds, sauf le dernier vers l'ouverture; par sa suture étroite, comme canaliculée; par son dernier tour très volumineux et très ample vers l'ouverture, ce qui donne à celle-ci une taille relativement plus développée que celle de l'H. chnoodia; par son test d'une teinte foncée marron, toujours recourert d'une couche de saletés terreuses fortement agglutinée.

\section{H. rypa Letourneux et Bourguignat, sp. nov.}

Testa anguste perforata, supra vix convexa, subtus convexo-turgida, subpellucida, sordide castaneo-lutescente, striatula ac breviter hirsuta et fere semper inquinata; spira subconvexa; apice pallidiore, lævigato; anfractibus 4 1/2 convexiusculis, rapide crescentibus, sutura impressa separatis; uttimo maximo, ad initium sat compresso, ad aperturam amplo ac rotundato-turgido, superne recto; apertura subobliqua, magna, paulo alliore quam latiore, mediocriter lunata; peristomate recto, acuto, ad marginen columellarem valde retrocedentem mediocriter dilatato; marginibus sat remotis; alt. 4 1/2, diam. $61 / 2$ millim.

Sous les pierres à Guelaat-es-Snam (Let.).

Diffère de l'II. persordida par' sa perforation plus étroite, par sa croissance plus rapide, par son dernier tour très grand, volumineux, rectiligne à son insertion, et offrant une ouverture encore plus ample que celle de la précédente, ourerture plus hante que large et remarquable par son bord coluniellaire fortement rétrocédent. Chèz cette espèce, le maximum de la convexité du dernier tour, à son origine, est tout à fait supérieur, et les bords non convergents sont relativement très distants.

\section{Zaritosi $H$. Berthier, sp. nov.}

Testa punctiformi-perforata, supra sat convexa, subpellucida, uniformiter cornea, argute striatula ac pilis brevissimis, sat distantibus, induta; spira convexa, ad summum obtusa (apice.lævigato); anfractibus 4 1/2 regulariter crescentibus, convexis, inter priores circa suturam profundam tumido-inflatis; ultimo vix majore, sat mediocri, rotundato, superne perlente sensim subdescendente; apertura obliqua, parum lunata, fere circulari; peristomate recto, acuto, modo ad marginem columellarem subdilatato ac patulo; alt. 4, diam. 6 millim.

Cette jolie Hélice, bien distincte de toutes celles de ce groupe, a été découverte sur les bords du lac de Bizerte (Berthier). 
I. Iasia Bourg. Mal. Alg. I, 1864, p. 188 , pl. XVIII, f. $26-29$.

Djebel Reças, Kef El-Djerdja, rochers de Guelaat-es-Snam (Let.).

$\$ 9$.

I. rupestris Draparnaud, Tabl. Moll. Fr. p. 71,1801 , et Hist. Moll. p. 82, pl. Vll, f. $7-9,1805$.

Porto-Farina, dans les anfractuosités des rochers (Bourg. 1867); très abondante à Foun-el-Goubel (Let.).

s 10.

I. Ienticula Ferussac, Prodr. n 154, 1821, et Hist. génér. Moll. p. 361, pl. LXVI, f. 1.

Sous les pierres dans les fossés des remparts du Bardo (Bouro. 1867); Mahamedia, Sidi-Aldul-Vached, Tozer (Kerim , 1873); La Galite; entre Sousa el Bir ElBuita (Gestro, 1876); El-Kef', Kef El-Djerdja, El-Aouina, amphithéâtre d'El-Djem, Henchir Kouti près hsar El-Mletameur, alluvions de rOued Sidi-Aïch, Gabès, Zarzis (let.).

\section{S 11 .}

H. pulchella Müller, Verm. Hist. II, p. $30,177^{\text {h. }}$

Bords de l'Oued Melah an seuil de Gabès (André, ${ }_{18} 8$ ); alluvions de l'Oued Sidi-Aiclı (Let.).

\section{s 12.}

H. Tanchouiama Buurguignut, in sched. 1867 .

Testa aperte pervieque umbilicala, depressa, opaca, crelacea, non nitente, omnino candida, argule strialula; spira leviter convexa; apice atro, exiguo: anfractibus 6 convexiusculis, lente usque ad ultimum crescentibus, sutura impressa separatis; ultimo relative maximo, amplo, compresso-rolundalo, superne perlente vix subdescendente; apertura parum obliqua, sublunata, Iransierse oblonga; peristomate recto, aculo, intus nitide labiato el undique leviter palulescente, ad marginem columellarem mediocriler dilatato; alt. 8 , diam. 15 millim.

Ruines de Haïdra (Let.).

Celle espèce, dídiée à M. Tauchon, chef du bureau arabe de Guelma en $186_{7}$, a élé découverte en Algérie sur les bas côtés de la route nationale de Bône à la Calle; elle fait partie de la série de l'll. ericetorum. Parmi les nombreuses formes de cetle série, celles qui ont le plus de ressemblance avec elle sont les H. obvia (Hartmann) de Suisse et des Provinces danubiennes, el notamment l'H. subobvia (Galland) des environs de Constantinople, dont elle diffère par sa coloration, par la direction descendante de son dernier tıur, par son péristome, et surtout par son ouverture oblongue dans le sens transversal. 


\section{$\S 13$.}

I. cespitum Draparnaud, Tabl. Moll. Fr. p. 92, 1801, et Hist. Moll. p. 109, pl. VI, f. $14-15,1805$.

Environs de Tunis (Bourg. 1867) ${ }^{(1)}$.

II. arenarum Bourguignat, Malac. Alg. I, 1864, p. 238 , pl. XXVII, f. t-9.

Environs de Kamart du côté des dunes (Bourg. 1867) ${ }^{(1)}$.

\section{U. calestis Letourneux et Bourguignat, sp. nov.}

Testa profunde pervie ac mediocriter aperte umbilicata, supra convexorotundala, solida, opaca, crelacea, sat nitente, subtiliter strialula, uniformiter subcærulescenti-lacteo-candida; spira sat elata, in tholo convexa; apice atro, minuto; anfractibus $61 / 2$ convexis, lente usque ad ultimum crescentibus, sntura impressa separatis; ultimo maximo, cylindrico, fere ex initio usque ad insertionem labri per totam anfractus involutionem descendente; apertura obliqua, vix lunata, exacte sphærica, intus pallide ochracea; peristomate recio, aculo, intus profunde albo-incrassato, ad marginem columellarem mediocriter reflexo; marginibus approximatis; alt. 15-18, diam. 21-25 millim.

Sur les rochers aux ruines de Haïdra (Let.).

Cette forme de la série des $H$. cespitum et arenarum ne peut être rapprochée que de la belle $H$. meteora Bourg. des hauts plateaux de Setif. Elle s'en distingue néanmoins par sa taille moindre (l'H. meteora a 19 millim. de hauteur sur 30 de diamètre); par sa spire plus élevée, mieux arrondie (celle de l'H. meteora est presque écrasée); par son dernier tour cylindrique et son ouverture sphérique (chez l'H. metcora, le dernier tour, tout en étant arrondi, est sensiblement plan supérieurement, et par cela même, plus largement dilaté, et l'ouverture, moins oblique, est très oblongue dans le sens transversal); enfin, par son ombilic beaucoup plus étroit que celui de l'H. meteora.

\section{$\$ 14$.}

Les Hélices de ce groupe semblent former une série spéciale aux contrées montueuses de la Tunisie qui avoisinent les frontières de la province de Constantine; ses direrses espèces présentent depuis la forme la plus déprimée et la plus largement ombiliquée ( $H$. Barrattci) jusqu'à la forme la plns conique et la moins ombiliquée (H. Bouderiesa). Malgré ces signes différentiels si dissemblables, toutes ces Hélices ont un singulier air de parenté; toutes sont blanches, avec un derniet. tour nuancé d'une teinte ocracée-rougeâtre, entouré d'une bande unique, étroite, sensiblement supérieure, d'un roux marron. Celte bande se montre identiquement semblable chez les six espèces qui composent ce groupe tunisien.

(1) M. le commandant Morlet (in Arch. miss. sc., 1881, p. 394) signale dans la plaine d'Hameimet une variété de $H$. cespitum. Nous doutons fort de l'exactitude de cette détermination. 
H. Barrattei Letourneux et Bourguignat, sp. nov.

Testa profunde ac late umbilicata (umbilico exacte pervio), depressa, supra parum convexa, cretacea, opaca, nitida, striatula, candida, in ultimo plus minusve subochracea et zonula angusta, rufo-castanea, leviter superiore, cincta; spira depressa, mediocriter convexa; apice atro, minuto; anfractibus 6 convexiusculis, regulariter ac parum velociter usque ad ultimum crescentibus, sutura impressula, in ultimo impressiore, separatis; ultimo inaximo, rolundato, superne fere ex initio usque ad insertionem perlente descendente; apertura obliqua, vix lunata, exacte circulari, intus candide nitidissima; peristomate recto, acuto, profunde incrassato, ad marginem columellarem validiore et superne vix dilatato; alt. 14, diam. 26 millim.

Cette belle Hélice, dédiće à M. Barratte, secrétaire du Président de la Mission de l'exploration scientifique de la Tunisie, est abondante entre El-Kef et Souk-el-Arba. En Algérie, elle a été constatée à Souk-Alıras et près de Bône, où l'on rencontre parfois quelques individus qui n'ont que 8 millim. de hauteur sur 14 de diamètre.

\section{Slouguia Letourneux et Bourguignat, sp. nov.}

Testa profunde, exacte pervie ac late umbilicata sicut apud præcedenteni, globoso-conoidea, cretacea, opaca, nitida, striatula, lactescenti-candida, in ultimo prope aperturam ochracea et zonula rufo-castanea, sat decolarata, circumornata; spira conoidea, producta; apice atro, exiguo; anfractibus 7 convexis, regulariter crescentibus, sutura impressa separatis; ultimo mediocriter majore, exacte rotundato, ad insertionem sat breviter descendente; apertura obliqua, sat lunata, sphærica, intus candido-lutescente; peristomate recto, acuto, intus incrassato, ad marginem columellarem vix dilatato; marginibus sat remolis; alt. 17, diam. 23 millim.

Cette espèce, découverte anx environs de Klanget-Slougui, se distingue de la précédente par sa lorme globuleuse-conoïle, par sa croissance régulière, par ses tours bien cylindriques, par son dernier peu développé, bien rond et offrant, à linsertion supérieure, une descente assez courte el sensiblement accentuće, par son ouverture plus échancrée, exactement sphéricue dans le sens verlical, landis que chez l'II. Barrattei l'ouverture est circulaire dans un sens légèrement transıerse. Dans les deux espèces, J'ombilic, à peu près semblable, est néanmoins chez jH. Slouguia un peu moins érasé ì son orifice.

I. arrara Letourneux et Bourguignat, sp. nov.

Testa profunde angusteque umbilicata, globosa, supra conica, opacula, nitente, striatula, lutescente aut Jactescenti-candidula, ac zonula rulocastanea cincta; spira producto-conica; apice atro, exiguo; anfractibus 6 1/2 convexiusculis, lente usque ad ultimum crescentibus, sutura impressula separatis; ultimo maximo, turgido-cylindrico, ad insertionem vix 
subdescendente; apertura fere verticali, sat lunata, ampla, semisphærica, intus pallide ochracea; peristomate recto, acuto, sat fragili, intus non incrassato, ad marginem columellarem dilatato; marginibus valde remotis; alt. 17 , diam. 22 millim.

Environs d'Aïn Bouderiès, el plus rare aux ruines de Haidra (Let.).

Cette forme, remarquable par l'étroitesse de son ombilic, se distingue encore de la précédente, par son test d'un diamètre moins grand, bien que de même taille comme hauteur, par sa croissance spirale lente jusqu'à son dernier tour qui est plus volumineux, plus renflé et plus haul que celui de l'H. Slouguia, par sa descente supérieure à peine accusée, par son ouverture verticale plus ample, plus échancrée, par son bord péristomal simple, assez fragile, non épaissi, plus dilaté à la columelle; enfin, par ses bords marginaux plus distants.

H. burella Letourneux et Bourguignat, sp, nov.

Testa anguste umbilicata (umbilico in ultimo leviter patulo), turgidodepressa, supra parum convexa, opacula, nitente, striatula, pallide lutescente aut sordide albida, ac zonula rufo-castanea plus minusve diluta circumcincta; spira convexa ; apice atro, minuto; anfractibus 6 convexiusculis, regulariter usque ad ultimum ac deinde rapide crescentibus, sutura inter superiores vix impressula, in ultimo impressa, separalis; ultimo relative maxims, amplo, dilatato, cylindrico-turgido, perlente e dimidio anfractus subdescendente; apertura fere verticali, sat lunata, semicirculari, altiore quam latiore, intus pallide subochracea; peristomate recto, acuto, profunde incrassatulo, ad columellam leviter dilatato; narginibus remotis; alt. 16 , diam. 23 , ap. alt. 12 , lat. 11 mill.

Aïn Boudeiriès (Let).

Cette Hélice est, de toutes les espèces de ce groupe, celle qui possède, relativement à sa taille, un dernier tour aussi développé en volume et en grosseur, ainsi qu'une ouverture aussi ample.

II. Khangetina Letourneux et Bourguignat, sp. nov.

Testa profunde, pervie et sat aperte umbilicata, globoso-subdepressa, supra sat convexa, leviter tectiformi, cretacea, opaca, nitida, strialula ac passim omnino lævigata, candida aut albo-lutescente, ad aperturam subochracea ac zonula rufo-castanea, plus minusve evanida, cincta; spira convexa, ex ultimo usque ad summum subconica, quasi tectiformi; apice atro, minuto; anfractibus $61 / 2$, lente usque ad ultimum, deinde velociter crescentibus (prioribus subplanulatis), sutura inter supremos lineari, ad ultimum impressa, separatis; ultimo magno, amplo, globoso-rotundato, ad insertionem lente descendente; apertura sat obliqua, parum lunata, fere circulari, intus pallide subochracea; peristomate recto, acuto, intus incrassato, ad marginem columellarem leviter reflexo; alt. 17; dian. 25 millim. 
Cetle nouvelle espèce, remarquable par sa spire conique-tectiforme, par sa suture superficielle entre les tours supérieurs, etc., vit, en compagnie de l'H. Slouguia, aux environs de Khanget-Slougui (Let.).

I. Boudriesa Letourneux et Bourguignat, sp. nov.

Testa profunde perforata, globosa, supra conica, sublus convexo-turgida, cretacea, opaca, nitente, striatula, supra candida, infra candidosublutescente, ac zonula rufo-caslanea cincta; spira producto-conica; apice atro, minuto; anfractibus $61 / 2$ convexis, regulariter crescentibus, sutura impressula separatis; ultimo magno, ad aperturam anpliore, globoso-rotundato, ad inserlionem recto, subtus circa perforationem subangulato; apertura sıbobliqua, parunı lunata, subrotunda, altiore quam latiore, inferne subangulosa, intus candida; peristomate recto, acuto, intus leviter incrassatulo; margine columellari mediocriter rellexo, fere recte usque ad angulum descendente; marginibus sat approximatis; alt. 17 , diam. 20 , ap. alt. 12 , lat. 10 millim.

Aïn Bouderiès (Let.).

Celte fornie caractérisée par sa petite perforation, par son test ghobuleux à spire conique-élevée, par son dernier tour subanguleux en dessous," par son bord columellaire descendant presque en ligne droite, etc., ne peut être confondue avec ancune de ce groupe. C'est celle qui de toutes possède l'ouverture la moins circllaire.

\section{$\$ 15$.}

Les qualre llélices suivantes font partie d'une série d'espèces (1) Irès nontbreuses, répandues en Mlgérie, en Espagne el même jusque dans le midj de la France. C'est principalement en Algérie que les formes de cette série offrent leur plus beau déreloppement.

Les Hélices tunisiennes de ce groupe sont de grandes Hélices ombiliquées, globuleuses-coniques (sauf deux qui sont déprimées), à test solide, élégamment zonulé et fortement sillonné par des striations ressemblant à des côtes.

II. Zitanica Letourneux et Bourguignat, sp. nov.

Testa profunde umbilicatis (umbilico leviter pervio, mediocriter in ultimo aperto), subgloboso-conoidea, solidula, subopacnla, nitente, robuste striala (striis sive costulis regularibus, densatis), rufo-subalbida (in prioribus (lecolorato-albida) ac zonulis rufis numerosis eleganter cincta; spira conico-convexa, ad summum sal acuta (apice atro, minuto); anfraclibus 7 , lente usque ad ultimum, deinde rapidius crescentibus (supremis leviter convexiusculis, cæleris convexioribus), sutura inter priores subimpres-

(1) Telles que les Helix Birinica, Agouniniana, mrtoporina (Bourg.), Djelfana (Pechaud), naperrina, Cartennensis (Let.), Redoni (Berthier), d'Algérie; Adolfi (Pfeiffer), stiparum (Rossm.), Arigoi (Bourg.), etc., d'Espagne et de France. 
sula, in ultimo impressa, separatis; ultimo majore, rotundato, nihilominus superne leviter declivi, subtus exacte convexo, ad insertionem sat breviter descendente; apertura obliqua, mediocriter lınata, subrotundata (margine superiore leviter recto-declivi), intus rufula cum zonulis apparentibus; peristomate recto, acuto, rufo-incrassato, iuferne subpatulo, ad marginem columellarem reflexo; alt. 15 , diam. 22 inillim.

Mèdinet Ziam près Zarzis (Let.). Cette espèce a été également recueillie, en Kabylie, à Agouni-el-Haoua (Let.).

Les zonules chez cette Hélice sont ordinairement au nombre d'une douzaine, rarement plus, quelquefois moins lorsque quel yues-unes d'entre elles se réunissent, comme il arrive pour les bandes supérieures. Ces bandes, lorsquelles sont réunies, sont ornées de fascies ou de maculatures moins foncées. Les inférieures sont presque toujours continues. Il en existe une très large et très foncée dans le trou ombilical. Sur la spire, les zones disparaissent (sauf dans les échantillons d'Agouni-el-Haoua) et sont remplacées par des vestiges de bandes ou par quelques maculatures.

\section{Lotophagorum Letourneux et Bourguignat, sp. nov.}

Testa profunde umbilicata (umbilico mediocri, in ultimo sat aperto), globoso-conoidea, solidula, nitente, regulariter (in ultimo irregulariter) robuste striata, albidula cun zonulis 6-10 rufo-nigrescentibus continuis; spira conica, subtectiformi, ad summum acuta (ap.ce atro, minuto); anfractibus 7 regulariter ac sat celeriter crescentibus, sutura impressula separatis; ultimo magno, turgido, rotundato, superne valido descendente; apertura perobliqua parum lunata, subovato-rotundata, intus subrulula cum zonulis apparentibus; peristomate recto, acuto, non incrassalo, ad marginem columellarem crassiore ac parum reflexo; alt. 20, diam. 25 millim.

Environs de Zarzis et collines le long de l'Oued Ghomrassen dans l'extrême sud de la Tunisie (Let.).

Cette espèce, indépendamment de sa croissance spirale différente, de la descente plus longue et plus accentuée de son dernier tour, de son ouverture plus oblique, de son péristome ni épaissi ni patulescent inférieurement, etc., se distingue notablement de la précédente par ses tours plus volumineux, plus gonflés, plus exactement ronds, ce qui donne à cette coquille une apparence globuleuse très prononcée. Chez l'H. Zitanica, les tours, d'un calibre plus faible, paraissent, par cela même, plus délicats.

II. meninxica Letourneux el Bourguignat, sp. nov.

Testa anguste umbilicata (umbilico profundo, non pervio nec aperto), globosa, nihilominus depressula, subopacula, nitida, argute robusteque striata, candida cum zonulis $4-12$ rufis et continuis; spira subdepressa, parum contexa, tamen subconoideo-tectiformi, ad summum acula 
(apice atro, minuto); anfractibus 7 convexiusculis, sat rapide crescentibus, sutura impressa separatis; nltimo magno, amplo, turgido, rotundato, ad insertionem lente ac sat breviter descendente; apertura obliqua, sat lunata, circulari, intus rufulo-subrosacea cum zonulis apparentibus; peristomate recto, acuto, intus valde labiato, inferne patulo, ad marginem colunellarem robusto ac sat reflexo; alt. 15 , diam. 23 millim.

İle de Djerba à Houmt-Souk, Ras El-Djerf, Sebkha El-Zerguin, Foum-Hallouf. Zarzis et plateau des Наouaïa (Let.).

Cette Hélice se distingue des deux précédentes par sa forme plus surbaissée, moins conique, par létroitesse de son ombilic, par son dernier tour plus globulenx, par son bord péristomal plus robuste, plus fortement épaissi, par sa croissance spirale plus rapide, etc.

II. mesembriea Letourneux et Bourguignat, sp. nov.

Testa anguste umbilicata (umbilico perforationi similis), globoso-depressa, supra parum convexa, solidula, subopacula, nitente, valide striata, albidula cum zouulis angustis, numerosis, rufulis ac sæpe evanescentibus; spira regulariter ac mediocriter convexa, ad summum perobtusa (apice rufo, minimo); anfractibus 7 convexiusculis, sat rapide crescentibus, sutura impressula separatis; ultimo maximo, anplo, globoso-rolundato, superne ad insertionem breviler vix descendente; apertura mediocriter obliqua ac lunata, ampla, subcirculari, intus rufulo-rosacea cum zonulis dilutis vix apparentibus; peristomate recto, acuto, intus vix incrassatulo, ad marginem columellarem subreflexo; alt. 14, diam. 24 millim.

Euvirons de Zarzis et de Bir El-Ahmar (Let.).

Cette Hélice differe de l'H. meninxica, la seule du groupe qui puisse lui être assimilée, par son ombilic plus étroit, par son test plus déprimé, par sa spire peu convexe, par son dernier tour plus volumineux, par son ouverture plus ample, moins échancrée, par son péristome plus délicat, non patulescent à la base et à peine réfléchi à la columelle.

\section{$\S 16$.}

Les H. Ammederana, IIaidrana et Birta appartiennent encore à un groupe de nombreuses espèces répandues de la Tunisie et de l'Algérie, où elles atteignent leur maximum de développement, jusqu'en Espagne et dans le midi de la France. Parmi elles nous citerons les $H$. Mantinica (Mabille), Panescorsi (Bérenguier), Marioniana (Bourg.), etc., de France; Devauxi, Kabyliana (Debeaux), Subkabiliana (Let.), Bidaensis, calopsis (Bourg.), etc., d'Algérie.

H. Ammederana Letourneux et Bourguignat, sp. nov.

Testa anguste umbilicata, depressa, supra subtusque fere æqualiter convexa, opacula, nitida, subtiliter striatula, lutescente aut albida, ac zonulis numerosis $(5-10)$ rufo-atris, continuis, eleganter cincta; spira 
parum convexa, depressa; apice atro, minuto; anfractibus 6 convexiusculis, sat rapide crescentibus, sutura impressula separatis; ultimo magno, rotundato, nihilominus leviter compresso, ad insertionem recto aut vix brevissime subdeflexo; apertura obliqua, parum lunata, ovata aut ut melius dicam subrotundata, intus sordide albidula cum zonulis apparentibus; peristomate recto, acuto, intus profunde valde labiato (labio robusto, candido ac splendido), ad marginem columellarem vix reflexo; alt. 9, diam. 16 millim.

Bois de Pins près des ruines de Haïdra (Let.).

H. Haidrana Letourneux et Bourguignat, sp. nov.

Testa anguste umbilicata, depresso-subglobosa, supra convexo-tectiformi, solida, opacula, nitente, striatula, albida cum zonulis 5 atrorufis, angustis, valde inter se distantibus, continuis aut passim subinterruptis; spira convexo-tectiformi, ad summum acuta (apice atro, exiguo); anfractibus 6 convexiusculis, lente usque ad ultimum deinde rapide crescentibus, sutura impressula separatis; ultimo magno, dilatato, rotundato, ad insertionem lente subdescendente; apertura obliqua, lunata, transverse oblonga, intus albida cum zonulis apparentibus; peristomate recto, acuto, intus valide labio splendide candido circumincrassato, inferne subpatulo, ad columełlam leviter reflexo; alt. 9, diam. 16 millim.

Ruines de Haïdra (Let.).

Cette Helice se distingue de la précédente par sa forme moins déprimée, par sa spire convexe-tectiforme assez élevée, par son dernier tour moins renflé, plus déclive aux abords de l'ouverture et pourvu, à l'insertion, d'une direction descendante plus longue et plus accentuée, par son ouverture oblongue, légèrement déclive, moins haute el plus allongée que celle de l'H. Ammederana.

II. Rirta Letourneux et Bourguignat, sp. nov.

Testa peranguste umbilicata, depressa, supra parum convexa, subopacula, nitida, subtiliter striatula, candidula, cum zonulis 5 atro-rufis continuis quarum 2 ad peripheriam et 3 inferæ graciliores; spira depressa, vic convexa; apice minimo, rufulo; anfractibus 6 , regulariter usque ad uttimum deinde velociter crescentibus, sutura impressula separatis; ullimo maximo, dilatato, subcompresso-rotundato, ad insertionem recto; apertura vix obliqua, lunata, transverse oblonga, non declivi, intus albida cum zonulis apparentibus; peristomate recto, aculo, intus profunde labio candido circumornato, inferne subpalulo, ad columellam subreflexo; alt. 8 , diam. 15 millim.

Ruines de Haïdra (Let.).

L'H. Birta diffère: $1^{\circ}$ de l'H. Ammederana par l'étroitesse de son ombilic, par sa spire plus déprimée, moins convexe, par ses zonules moins nombreuses et plus 
distantes les unes des autres, par son dernier tour non exaclement arrondi mais subcomprimé; ce qui le fait paraitre transversalement plus dilaté, par son ouverture oblongue dans le sens loorizontal; $2^{\circ}$ de l'H. Haidrana également par son ombilic et par sa spire à peine convexe (celle de I'H. Haidrana est convexe-ćlevée et tectiforme), par son dernier tour rectiligne ì linsertion, offrant de plus un contour lécrèremeut sulvomprinıé, arroudi-dilaté dans le sens horizontal el non déclive; cnfin, par son ouverture transversalement oblongue, et non oblongue dans un sens incliné, comme celle de l'H. Haidrana.

Chez les trois Hélices de cette série, le príristome, très aigu, est cerclé en dedans par un encrassement d'un blanc éclatant. Ciet encrassement, profondément situé sur la paroi interne supérieure de l'ouverture, devient peu à peu unarginal en se rapprochant de li columelle.

\section{\$ 17.}

Les espèces de ce groupe sont toutes des formes salıariemes. Une, l'II. Molitana (Let.) n’a encorc élé constatée que daus le Sahara algérien aux environs de Metlili; les autres sont de l'extrême Sud tunisien.

IT. Frativiana Letourneux et Bourguignat, sp. nov.

Testa perforata : globosa, fere æejue convexa supra ac sublus, opacula, subcretacea, parum nitente, eleganter ac regrulariter (in ultimo grosse) striati, candida cum zonulis anguslis lere evanidis ac sæpe vix perspicuis; spira convexa sat elala; apice minuto; anliactibus $61 / 2$ convexiusculis, regrulariler nspue ad ullimum ac deinde celeriler crescentibus, sulura impressula separitis; ultimo relalive maximo, rotundato, ad inserlionem recto; apertura obliqua, sat lunila, semirotundala, iulus albida; peristomate recto, acuto, profunde vix incrassatulo, ad columellam modo superne dilatato; alt. 15 , dians. 22 millim.

Environs de Fratis (Let.).

\section{Tafermica Letourneux et Bourguignat, sp. nov.}

Testa perforata, subglobosa, sıperne convexiore quam subtus, subopacula, vix nilente, valde striala (striis sive costis validis ac regularibus), albida cum zonulis 5-6 evanidis; spira produclo-convexa, subconoidali, ad summum subacuta (apice atro, minulo); anfraclibus 7 convexiusculis, regulariter crescentibus (ultimo nilnilominus ad aperturam ampliore), sulura impressa separatis; ultimo majore, ad initium obscure subangulato, subrotundato, ad aperturam superne declivi, ad inserlionem lente descendente; apertura obliqua, lunata, semiorata, superne subrecte declivi; peristomate recto, acuto, intus subincrassatulo, ad columellam mediocriter rellexo; alı. 15 , diam. 20 millim.

Bords des Oued Taferma el Cherb (Let.). Dans les oasis au sud de Biskra (province de Constantine), on rencontre une forme de taille moindre (haut. 13 , diam. 18 millinı.) 
Cette Hélice se distingue de la précédente par sa forme moins globuleuse, par ses tours plus délicats, moins renflés, moins exactement cylindriques, puisque le dernier, à son origine, offre une légère apparence subanguleuse, par son test plus régulièrement et plus fortement strié, par son dernier tour moins gros, lentement descendant à l'insertion et sensiblement déclive à l'ouverture qui est moins ample et ovalaire dans un sens incliné.

1. Mezessaria Letourneux et Bourguignat, sp. nov.

Testa perforata, parvula, globoso-conoidea, subopacula, parum solida, nitente, striatula, sublutescente-albida cum zonulis 10-12 rufulis, angustis ac continuis; spira producto-convexa, conoidea, tectiformi; apice rufo, minuto; anfractibus 6 parum convexiusculis, regulariter crescentibus, sulura fere lineari, in ultimo impressula, separatis; ultimo majore, globoso-rolundato, superne perlente subdescendente; apertura parum obliqua, lunata, semisplırica; peristomate recto, peracuto, fragili, intus non incrassato, ad columellam superne leviter reflexo; alt. 10 1/2, diam. 14 millim.

Oued Mezessar (Let.).

Cetle espèce globuleuse, conoïde, de taille médiocre, à péristome mince, très tranchant, non épaissi, à ouverture semi-sphérique, etc., ne peut être confondue avec les deux précédentes.

\section{\$ 18.}

E. Rardoensis Boirguignat, Hist. Malac. Tunis, p. 18, pl. I, f. 19-21, 1868.

Le Bardo, sur les pelouses. Revers des fossés des jardins de la Manouba, près le Bardo (Bourg. 1867). Cette Hélice vit également en Algérie anx environs de Bougie.

\section{\$ 19 .}

II. axiotheata Bourguignat, in sched. 1872 .

Testa profunde angusteque umbilicata (umbilico perforationi simili), depressa, fere æque convexa supra ac sublus, solidula, nitida, valide striata, quasi costulata (costulis regularibus, validioribus supra quam sublus). lutescenti-albida, ac supra zonula unica castaneo-straminea fata eleganter albide picta, et inferne zonulis mumerosis dilutis, modo prope aperturam perspicuis, circumcincta; spira mediocriter convexa, in tholo rolundata; apice atro, exiguo; anfractibus 6 vix convexiusculis, regulariter crescentibus, sulura fere lineari separatis; ultimo mediocri, subangulato (angulo leviler supero, ad aperturam subevanescente), supra mediocriter convexo, infra convexiore, ad insertionem breviter deflexo; apertura leviter obliqua, tunata, semiorata; peristomate recto, acuto, intus valide albido-incrassato, inferne patulo et ad columellam crassiore el reflexo; alt. 6, diam. 10 millim. 
Le Bardo, près Tunis (Bourg. 1867).

Cette espèce appartient à la série de l'II. Oranensis, dont les principales formes sont les H. pelidna, Gilvana, abomera, choreta (Bourg.), Gouini (Deb.), Doueranensis (Pechand), sphcerita (Hartm.), etc., d'Algérie; Gregorioi (Bouro. .), derelicta (Villa), de Sicile; nautica (Locard), de France.

$\$ 20$.

h. eucalla Hagenmiiller, sp. nov.

Testa profunde ac sat aperte perforala, depressa, supra convexa, subtus convexiore, opacula, cretacea, striatula, candida; spira in tholo convexa, parum producta, ad summum perobtusa (apice atro, minutissimo); anliactibus 6 vir convexiusculis, leviter declivi-planulatis, lente usque ad ultimum, deinde rapidius crescentibus, sutura lineari, in ultimo impressula, separatis; ultimo magno, ad initium compresso-angulato, ad aperturam amplo, rotundato, ad insertionem recto; apertura parum obliqua ac lunata (marginibus approximatis), ampla, fere circulari; peristomate recto, valide albo incrassalo, leviter patulescente, ad columellam parum dilatato; alt. 7 1/2, diam. 12 millim.

Colline à Beja (Hagenmüller).

Celte espece est tout à fait semblable comme forme el comme aspect à l'Helix Langloisiana (Bourg.) des bords de la Mer Morte. Elle en diffère, néanmoins, par sa taille plus forte, par sa perforation hien plus ouverte, par ses tours plus nombrenr, dont les supérieurs sont plus plans, par sa suture superficielle, et notamment par sa surface finement striolée, et non sillonnée, comme chez l'H. Langloisiana, de stries crispulcées, fortes, saillantes, prenant sur l'angulosité du dernier tour l'apparence de petites éminences tuberculeuses.

I. Gery villenvis Bourg. Paléont. Alg. p. 65, pl. IlI, f. 13-1 7, 1862, et Malac. Mlg. I, p. 208 , pl. XXI, f. 1-6, 1864.

Fossés du Bardo et de la Manouba, près Tunis (Bourg. 1867); Tebourba (André, 1878 ).

L'II. Langloisima (Bourg.), de Syrie et de Perse, fait partie du groupe de cetle Hélice.

\section{\$ 21.}

II. Saharica Bourguignat in Ancey, in Bull. Soc. mal. Fr. I, 1884, p. 170.

Cette jolie espèce des oasis d'El-Kantara et de Mchounech près Biskra, a été recueillie dans le sud de la Régence, entre Bir el-Kelba et le Chotl El-Gharsa (Duveyrier).

E. ischurostoma Bourguignat in Ancey, in Bull. Soc. mal. Fr. I, 1884, p. 171.

Entre le Cap Roux et le Cap Negro ì l'ouest de Bizerle (Berthier).

Ces deur Hélices rentrent dans une série spéciale d'espèces, dont la plupart sont particulières au nord de l'Afrique, à la Sicile, à l'ttalie, etc., telles que les 
H. Nahrouasselina, mucini, cacodiella (Bourg.), d'Algérie; amaura, amauronella, Bivonce, Sanvitoensis, bertula, narellina (Bourg.), etc., de Sicile et d'Italie.

\section{$\$ 22$.}

A l'exception d'une espèce espagnole (l'H. Arnusi, Servain, Moll. Esp. p. 97, 1880 ), toutes les autres dont nous allons signaler les caractères sont de la région des Chott du Sahara.

H. bradybaena Letourneux et Bourguignat, sp. nov.

Testa perforata, subgloboso-conica, subopacula, nitente, subtiliter striata, candida cum zonulis 5-10 translucentibus, quasi oleatis; spira producta, convexo-conica, ad summum subacuta (apice rufo, lævigato ac sat robusto); anfractibus 6 convexis, regulariter crescentibus, sutura impressula separatis; ultimo majore, ad initium subcompresso, ad aperturam leviter ampliore ac rotundato, superne ad insertionem recto; apertura parum obliqua, lunata, semirotundata; peristomate acuto, intus crasso, valide labiato, undique patulo, ad columellam reflexo; alt. 9 , diam. 12 millim.

Environs de Tozer (Let.).

H. terricola Bourguignat, in sched. 1880 .

Testa perforata, subglobosa, sat translucida, nitente, argutissime striatula, albidula cum flammulis translucide oleatis; spira convexa, producla, subconoidea; apice minuto, rufulo; anfractibus 7 convexiusculis, regulariter usque ad ultimum, deinde rapidius crescentibus, sutura impressula separatis; ultimo magno, dilatato, rotundato, ad initium subcompressiusculo, ad insertionem recto; apertura subobliqua, ampla, lunata, semirotunda; peristomate subochraceo, crasso, valide labiato, patulo, ad columellam robusto late reflexo; alt. 9 , diam. $12211 / 2$ millim.

Tozer (Duveyrier).

Cette forme diffère de la précédente par son test plus délicat, moins conoïde, par son mode de coloration, par sa croissance spirale moins régulière, par son dernier tour plus gros, plus volumineux, par son ouverture plus ample, par son péristome subocracé plus épais, plus fortement labié, dont le bord columellaire, un peu moins descendant, est sensiblement plus cintré.

\section{II. galeomma Bourguignat, in sched. 1880 .}

Testa anguste perforata, globosa præsertim subtus, supra sat exigue conoidea, infra convexo-turgida, opacula, nitente, argute striatula, omnino candida; spira producto-conoidea; apice minimo, rufulo; anfraclibus 6 convexis, regulariter ac sat lente crescentibus, sutura impressa separatis; ultimo maximo, ad initium obscure subangulato, ad aperturam 
turgido-rotundato, ad insertionem recto; aperiura obliqua, sat valide lunata, semirotunda, leviter altiore quam latiore; peristomate albo-labiato, patulo, ad columellam non reflexo; alt. 8, diam. 10 millim.

Eutre Tozer et Nefta (Duveyrier).

Hélice remarquable par l'exiguité de sa spire conoïdale, comparée au volume de son dernier tnur, par son ouverture très échancrée, légèremenl. plus haute que large et ressemblant à un croissant, par son péristome ni rétléchi, ni dilaté à sou hord columellaire, mais simplement patulescent sur tout son contour, par son test d'un blanc uniforme sans bandes ni flammules Iransparentes, par sa perforation très étroite, offrant ì la columelle une légère direction rectiligne qui n'existe pas chez les deux précédentes par suite de la dilatation et de la réflexion péristomale.

II. aeglia Letourneux et Bou"guignat, sp. nov.

Testa anguste perforata, angulata, supra conoidea, subtus convexa, oprcula, nitente, subliliter striatula, omnino candida ant rarius zonulis 3 -5 rufo-dilulis circumornala; spira producta, conico-tectiformi, ad summum sat acula (apice minuta, ruliulo); anfractibus 6 supra vix convexiusculis, potius subplanulatis, anģulatis (angnlo suturam sequente), regulariter crescentiluss, sulura fere lineari separatis; ultimo majore, angalato (angulo ad aperluram evan scente), convexiore infra quam supra, arl insertionem reclo; apertura mediocriter obliqua, sat lunata, semirolundata; peristomate albo-iurrassato, und.que patulo; margine columellari robusto, rollexo ac sal subrecte descendeute; alt. 8, diam. 10 millim.

lintre Tozer et Kriz (Let.).

Cette espèce conoïde, à tours anguleux, est très distincle de celles qui prérèdent.

\section{II. argoterma Letourneur et Bourguignat, sp. nov.}

Testi profunde reiforata, glohosa, sat clata, subopacula, nitente, argute stritula, caudida cum flamulis ix perspicuis, dılulis ac translucidis; spi a producta, valde conexa, ad summum oblusula; apice rululo, exigyur; an.ractihus 6 convexiusculis, lente usque ad ultimum, dsinde rapide crescentibus, sutura impressula separatis; ultimo magno, rotundato, turgido, superne recto; apertura vix obliqua, lusata, semiroturidata; peri lomate albo-labiato, undique subpatulo, ad columellam teneram vix relleso; alt. 10 , dian. 11 millim.

Tozer (Let.).

Celie forme, remarquable par sa enfuille presque aussi hante que large, par son dernier tour globuleux, bien arıondi dès son origine, par son bord c.luntellaire relativement mince et à peine rélléchi, ne peut être confundue avec aucune des précédentes. 
H. briarzea Bourguignat, sp. nov., 1880.

Testa anguste perforata, subglobosa, crassa, cretacea, nitente, argute striatula, omnino candida; spira producto-convexa, subconica; apice rufo, minimo; anfractibus 6 convexiusculis, lente usque ad ultimum, deinde leviter rapidius crescentibus, sutura impressula separatis; ultimo mediocriler majore, obsolete subangulato, subcompresso-rolundato, ad insertionem leviter vix subdescendente; apertura obliqua, lunata, semiovata; peristomate candido, percrasso, robuste labiato, patulo ac subreflexo; margine columellari dilatato; alt. 8, diam. 11 millim.

Environs de Kriz (Duveyrier).

Cette Hélice est très caractérisée par l'épaissseur de son test, par son énorme bord columellaire snbréfléchi, par son ouverture semi-ovalaire, par son dernier tour sensiblement comprimé et légèrement descendant à son insertion.

\section{I. agrarica Bourguignat, sp. nov., 1880.}

Testa profunde ac sat anguste umbilicata, subgloboso-conoidea, obscure subangulata, supra conoidea, subtus convexa, opacula, subcretacea, nitente, striatula (in ultimo grosse striata), candida; spira producta, conoidea, ad summum subacuta; apice subprominente, sat valido; anfractibus 6 convexiusculis, lente crescentibus, sutura impressula separatis; ultimo mediocri, subangulato (angulo ad aperturam obsoleto), supra convexo, infra convexiore, ad marginem recto aut parum subascendente; apertura vix obliqıa, lunata, semirotunda; peristomate crasso, valde albo-labiato, undique patulo, ad columellam vix reflexo; alt. 7 , diam. 9 millim.

Environs de Nefta (Duveyrier).

Cette espèce se distingue de l'H. briarcea par sa taille moindre, par son test moins épais, par ses striations plus fortes, grossières sur le dernier tour, par sa spire conoïde plus étriquée, à sommet sensiblement proéminent, par son dernier tour moins volumineux, plus comprimé, par son péristome moins épais, non réfléchi. simplement patulescent, par son bord columellaire non dilaté, enfin par sa perforation ombilicale plus ouverte.

\section{II, careoraea Letourneux et Bourguignat, sp. nov.}

Testa profunde angusteque umbilicata, angulata, supra conico-producta, subtus convexa, solida, cretacea, nitente, argute striatula, candida cum zonulis 5-6 translucide oleatis, subdilutis, quarum superiores 2, cæieræ inferiores; spira producta, conico-tectiformi; apice rufulo, læıigalo, sat robusto; anfractibus 6 convexiusculis, lente crescentibus, sutura impressa separatis; ultimo mediocri, angulato (angulo ad aperturam subevanescente), supra convexiusculo, infra convexiore, ad aperturam subrotundato, ad insertionem recto; apertura vix obliqua, lunata, semiro- 
tundata, crassitudine peristomatis angustata ; peristomate percrasso, valide robusto ac valde incrassato, undique patulo, ad columellam subreflexo; alt. 7 , diam. 9 millim.

Cette espèce, des environs de Tozer, est, parni les Hélices de cette série, celle qui a le test le plus épais et le péristome le plus fortement épaissi. L'épaisseur péristomale dépasse un millimètre, ce qui est relativement énorme ponr une coquille de cette taille.

\section{II. amicula Bourguignat, sp. nov., 1884.}

Testa profunde ac sat aperle umbilicata, subolobosa, producto-subconica, subpellucida, sat tenera, nitente, subliliter striatula, candidula, ad peristoma ochracea, cum zonulis interruptis, quasi flammulatis, translucide oleatis et vix perspicuis; spira producta, conoidea, ad summum sat acula (apice rnlo, minuto); anfraclibus 6 convexiusculis, lente crescentibus, sutura impressa separatis; ultimo mediocriter majore, rotundato, ad aperturam declivi, ad insertionem breviter deflexo; apertura vix obliqua, lunata, semiovato-rolundata in directione declivi; peristomate reclo, aculo, ochraceo, intus prolunde luteo-labiato, non patulo, modo ad columellan leviter dilatalo; alt. 8 , diam. 9 millim.

Environs de Tozer, de Kriz (Duveyrier) el de Gabès (Let.).

Hélice renarquable par son test délicat, sensiblement transparent, par son dernier tour lurè̀vement délléchi ì l'insertion, prar son ouverture semi-ovale-arrondie ‘lans un sens obliqueneut incliné de lant en bas, par son péristome ocracé, droit, très tranchant et profondément labié.

II. amphibola Bourguignat, sp. nov., 1880 .

Testa punctiformi-perforata, angulata, lere aque conoidea (præter ad aperturam) supra ac subtus, solida, opacula, nitente, subtilissime strittula, candidula cum zonulis numerosis translucide oleatis vix perspicuis; spira breviter conica; apice rufulo, minuto; anfractibus 5 1/2 fere subplanulatis, carinatis (carina suturam fere linearem sequente), lente usque ad ulimum, deinde paulo rapidius, crescentibus; ultimo magno, valide anģulato (ingulo ad peristoma evanescente), ad aperturam dilatato-globoso, ad insertionem recto; apertura subobliqua, valde lunati, semirolunda, panlo altiore quam latiore; peristomate recto, crasso, valide albolabiato, subpatulo; margine columellari subrecte descendente ac leviler rellexiusculo; marginibus valde remotis; alt. 6, diam. 9 millim.

Cette forme, des alentours de Kriz (Duveyrier), caractérisée par sa forte angulation, par son test conoïde en dessus ct subconoide en dessons, sauf vers l'onverfure, où il devient bien rond, par son ouverture très échaucrée, par son bord columellaire sensiblement rectiligne, par sa perforation ponctiforme, etc., ne peut être confondue avec aucune de cette série. 
H. ambloxa Letourneux et Bourguignat, sp. nov.

Testa profunde aperteque perforata, angulata, conoidea, sat elata, subtranslucida, nitente, argutissime striata atque uniformiter candida; spira producta, conica; apice rufulo, minimo; anfractibus 6 vix convexiusculis, lente crescentibus, sutura fere lineari separatis; ultimo mediocriter majore, angulato (angulo ad peristoma obsoletissimo), convexiore infra quam supra, ad insertionem leviter lente subdescendente; apertura obliqua, lunata, semiovato-rotunda in directione leviter declivi; peristomate recto, acuto, vix incrassatulo, ad columellam valde dilatato; marginibus approximatis; alt. 7 , diam. 9 millim.

Son test délicat, son bord péristomal mince à peine bordé, très largement réfléchi seulement à la columelle, sa spire conique élevée, son dernier tour lentement descendant à l'insertion, ses bords marginaux rapprochés, son ouverture semi-ovalaire-arrondie dans une direction inclinée de haut en bas, etc., distinguent suffisamment des autres cette espèce que l'on rencontre sur les collines sablonneuses des alentours de Tozer (Let.).

\section{H. anasia Bourguignat, sp. nov., 188 o.}

Testa anguste perforata, depressa, valde angulata, fere æque convexoconica (præter ad aperturam) supra ac subtus, subopaca, nitente, argute striatula, candidula cum flammulis superne et zonulis numerosis inferne translucide oleatis vix perspicuis; spira depressa, obtuse conica; apice rufulo, minuto; anfractibus 5 vix convexiusculis, regulariter crescentibus, sulura impressula separatis; ultimo majore, angulato (angulo ad peristoma evanescente), ad aperturam rotundato, ad initium convexiore infra quam supra, ad insertionem recto ac circa perforationem turgido; apertura obliqua, lunata, semirotunda; peristomate recto, acuto, intus candido, labiato, subpatulo, ad columellam subdilatato; marginibus remotis; alt. 5, diam. 7 millim.

Cette Hélice, des environs de Kriz (Duveyrier) est une forme déprimée, très anguleuse, presque aussi convexe en dessus qu'en dessous. Son dernier tour est sensiblement renflé autour de la perforation. On remarque sur la paroi aperturopariétale du dernier tour un renflement qui a l'air de correspondre à un léger gonflement subtuberculeux de l'incrassement péristomal.

\section{II. goniogyra Bourguignat, sp. nov., 1880.}

Testa profunde angusteque perforata, depressa, conica, supra tectiformi, subtus subconico-convexa præter ad aperturam, opacula, nitente, subtilissime striatula, candidula cum zonulis vix perspicuis translucide oleatis; spira depressa, breviter conica, tectiformi; apice rufo, minuto; anfractibus declivi-planulatis, carinatis (carina suturam linearem sequente), lente crescentibus; ultimo mediocri, valde angulato, ad initium quasi ca- 
rinato, ad aperturam obscure subangulato ac subrotundato, ad insertionem lente descendente; apertura obliqua, parum lunata, subovata; peristomate recto, acuto, incrassatulo, ad columellam reflexo; alt. 5, diam. 7 millim.

Environs de Kriz (Dureyrier).

La direction descendante accentuée du dernier tour commence presque à moitié de la circonvolution. Lionverture, faiblement échancrée, est ovalaire dans une direction obliquement descendaute de gauche à droite. Les tours, fortement carénés, inclinés-tectiformes en dessus, s'accroissent arec lenteur.

H. Concholenca Letourneux et Bourguignat, sp. nov.

Tista perforata, depressa, carinata (carina candidissima), fere æque conoidea supra ac subtus, subpellucida, nitente, argutissime striatula (striis supra validioribus), candidula ac zonulis angustissinis translucide oleatis vix perspicuis infra ormata; spira depressa, breviter tectiformiconica; apice rufo, tuinuto; anfraclibus 5 carinatis (carina suturam linearem sequente), subplanulatis, sat celeriter crescentibus; ultimo carinato, compresso, contexiore infra quam supra, ad peristoma subrolundato, circa perforationem fumido, ad insertionem recto; apertura subobliqua, lunata, subrotundati, leviter extérne subangulosa; peristomate recto, acuto, intus incrassato, ad columellam subrellexo; columella brevi, subrecte descendente; marginibus renotis; alt. 5, diam. 7 mylim.

Environs de Tozer (Let.).

H. vivida Hagenmüller, sp. nov., 1881 .

Testa anguste perforata, ghobosa, opacula, nitente, valide striata, albida et superne circa suturan flammulis atris intermptis exacteque inter se distantibus ac \%onuli atra angusta mediana, arcte interrupta, eleganter ornata; spira producto-convexa, ad summum obtusa (apice lavigato, rufulo, sat magno); anfraclibus 6 convexiusculis, regulariter cresceutibus, sutura impressa separatis; ulli: o mediocri, subrotundato, ad insertionem lente descendente; apertura subobliqua, lunata, semioblongo-rotundata; peristomate recto, acuto, intus candido-labiato, undiyue vix patulescente et ad columellam subreflexo; alt. 7 1/2, diam. 9 1/2 millim.

Anfractuosités des rochers près le Cap Negrne entre le Cap Roux et Bizerte (Berthirr). Le type de cetle Hélice est abondant aux rocliers de Calla Vouta près le Cap Rosa entre Bòne et la Calle (Hagennuiller).

Lill. vicila fait partie d'un groupe d'espèces littorales, découvertes par le docteur Hagenmüller. Les formes connues, sans compter celle que nous venons de décrire, sont les H. vetara, venustula, vulpina et aviriosa (Hagenmüller). 
H. dexia Bourguignat, in sched. 1881 .

Testa punctiformi-perforata, subturrito-globosa, opacula, nitida, argute striatula quasi passim polita, uniformiter candida; spira valde producta, subconoideo-convexa, ad summum subacuta aut subobtusiuscula (apice atro, splendente, lævigato); anfractibus 6 vix convexiusculis, regulariter crescentibus, sutura impressula ad ultimum impressiore separatis; ultimo turgido-rotundato, superne declivi-convexo, ad insertionem breviter subdeflexo; apertura obliqua, lunata, semiovato-rotundata in directione oblique verticali, intus omnino aurantiaca; peristomate recto, acuto, sal lenui, inlus profunde incrassaiulo, ad columellam modo subreflexo; marginibus remotis; all. 9, diam. 10 millim.

Sur les collines sablonneuses entre le Ras El-Munchibar et le Ras El-Keroum à l'ouest de Bizerte (Berthier).

Cette Hélice a été également découverte en Sicile, ainsi qu'en Algérie entre Bône et.la Calle (Let.).

\section{IT. Neftana Bourguignat, sp. nov., 1880.}

Testa profunde perforata, subdepresso-globosa, opacula, nitente, argutissime striatula, uniformiter candida ; spira mediocriter convexa, nihilominus sat producta, ad sumnum obtusa (apice minuto, rufo); anfractibus 6 convexis, lente usque ad ultimum deinde rapidius crescentibus, sutura sat impressa separatis; ultimo magno, ad initium valide angulato (angulo ad aperturam evanescente), prope peristoma subrotundato, ad insertionem recto; apertura subobliqua, lunata, semirotunda, intus albida; peristomate crasso, valide labiato, subpatulescente, ad columellam sat reflexo; alt. 7 , diam. 9 millim.

Cette Hélice très distincte de la précédente vit aux environs de Nefta (Duveyrier).

- Ces deux formes tunisiennes appartiennent à la série de la vraie $H$. rufolabris (Benoit) de Sicile, série qui comprend non seulement l'H. mfolabris, mais encore les H. spernosa, Palizzolii et Luidgï (Bourg.) de Sicile, et l'H. biacta (Ancey) de Barcelone.

\section{ऽ25.}

Les espèces qui suivent ont toutes des coquilles globuleuses ressemblant à de petites boules; elles font partie d'un groupe d'Helix très nombreux, largement représenté dans le nord de l'Afrique. Les formes de ce groupe, sans compter les tunisienues, dont nous allons donner les caractères distinclif's, sont les H. Bertini (Bourg.) de France; soladonica, sparticola, rachgonica, adoulana; 
(estivalis ${ }^{(1)}$, Lacertarum, Candiotella, vacuensina, gitona, cyclestha, sebana, acromata (Bourg.), d'Algérie et du Maroc; Perregeauxi (Pechaud); Afirana, irvama, Berlieropsis, Onesserana (Letourneux); Berlieri (Morelet); Zahresina (Berthier), d'Algérie, etc.

I. Candiota Friwalshy et Mousson, Coq. Bellardi, p. 10, 1854 .

C'est cette espèce qui était généralement admise sous l'appellation d'Helix turbinata (Jan) (2). D’après Mousson, le nom de turbinata de Jan doit être appliqué à l'H. Aradasi (Mandralisca) de Sicile. Un grand nombre d'auteurs modernes ont adopté cette opinion, et comme, faute de preuves contraires, il nous est impossible de nous inscrire en faux contre celte manière de voir, nous appliquons le nom d' $H$. Candiota à l'ancienne 1 . turbinata des auteurs (non Jan).

Celte llélice est excessivement abondante dans toule la région méridionale de la Régence, où on la rencontre aux environs de Mehedia, d'Aïn-Cherichira près Kerowan, de Sfax, de Ras El-Djerf vis-à-vis de Djerba, et aux alentours de la principale ville de cette île, Hount-Souk (Let.).

Lill. Candiota, si répandue dans le sud de la Tunisie, est une espèce de Crète (de là son nom), quj a été constatée dans presque loutes les îles de l'Archipel et uême jusqu'en Syrie et en Asie Mineure.

C'est sous l'appellation d'Helix Durieni que le docteur Issel ${ }^{(3)}$ et le commandant Horlet ${ }^{(4)}$ ont signalé cette espèce dans l'île Djerba et à l'embouchure de l'Oued Helah (André, $\mathbf{1}^{8} 78$ ) près Gabès.

\section{H. psammathaea Letourneux el Bourguynat, sp. nov.}

Tesla punctiformi-perforata, globoso-sphreroidea, supra mediocriler convesa, solidula, subopacula, striatula (in ultimo grosse), candidula cum flammulis oleato-sublranslucidis, aul subochracea cum flammulis intentioribus; spira mediocriler convexa, ad summum oblusa (apice rufulo, lavigato); anfraclibus 5 sat convexis ac rapide crescentibus, sutura impressa separatis; ultimo maximo, inflato-globoso, exacle rolundato, cirea perforationem sape subturgidulo, ad insertionem recto; apertura subobliqua, lunata, ampla, semirotundata, paulo altiore quam latiore; peristomale recto, acuto, incolorato aut rufo-ochraceo, non aul vix incrassatulo, ad columelian modo subreflexo; alı. 8, dians. 9 1/2 millim.

Environs de Sfax, de Mehedia, de Zarzis et d'Houmt-Souk dans l'île de Djerba (1.et.).

Celte espèce, qui est à l'Hélice précédente ce qu'est l'H. Zahresina à l'H. Bertieri, se distingue nettement de l'H. Candiota, la seule espèce qui puisse lui être assimilée, par sa forme non globuleuse-turbinée, mais sphéroïde, par sa spire surbaissée,

(1) Helis graphica, Morelet, Moll. Maroc, 1880 , non H. graphica Hartmann, 1844.

(2) Mantissa, p. 2, 1832 , et Pfeiffer, Mon. Hel, viv. I, 1848, p. 155.

(3) Moll. croc. Viol. in Ann. mus. civ. Gen. 1880, p. 268.

(9) Coq. Chotts, in Arch. miss. sc. 1881, p. 394 . 
par son dernier tour relativement plus volumineux, plus renflé, plus haut, par son ouverture plus ample surtout en hauteur, par son bord péristomal mince, à peine épaissi, par ses bords marginaux sensiblement plus distants, etc. .

\section{$\$ 26$.}

H. Durieni Moquin-Tandon, mss. in L. Pfeiffer, G. Helix ( $2^{\mathrm{e} e ́ d i t . ~ C h e m n i t z) ~} \mathrm{n}^{\circ} 36 \mathrm{o}$, pl. CVIII, f. 9-10, et Mon. Hel. viv. I, p. 441, 1848 .

L'H. Durieui du docleur Issel et du commandani Morlet, signalée dans le sud de la Régence, doit être rapportée, ainsi que nous venons de le dire, à l'H. Candiota. La vraie $H$. Durieui est une forme essentiellement littorale qui n'a encore été réellement constatée que sur les dunes entre Bône et la Calle, et, en Tunisie, sur celles voisines de l'embouchure de la Guerah Tindja près de Bizerte (Berthier).

I. psammoica Morelet, in Journ. conch. II, 1851 , p. 356, pl. IX, f. 11.

Cette Hélice est distincte de l'H. contermina ${ }^{(1)}$ de Corșe, avec laquelle quelques auteurs étrangers ont voulu la confondre.

L'H. psammoica, très abondante en Algérie sur les plantes salines des environs de la Calle, a été rencontrée en Tunisie aux alentours de Bizerte (Berthier), d'Hamman-el-Lif, de Kelibia, de Mehedia, ainsi que sur les dunes de Zougag et sur celles entre Nebeul et Menzel-Temim (Let.).

I. psammrecella Letourneux et Bourguignat, sp. nov.

Testa punctiformi-perforata, obtuse producto-subconoidali, opacula, nitente, valide striata sive subcostulata, uniformiter candida aut flammulis atris irregulariter dispositis eleganter variegata; spira elevato-oblusa, subconoidali; apice obtuso, valido, atro et lævigato; anfiactibus 6 convexis, regulariter crescentibus, sutura mediocriter impressa separatis; ultimo mediocri, rotundato, ad insertionem breviter subdeflexo; apertura perobliqua, sat parvula, lunata, semisphærica; peristomate recto, aculo, intus leviter labiato, ad columellam dilatato-reflexo; marginibus sat approximatis; alt. $61 / 2$, diam. 6 millim.

Hammam-el-Lif, dunes de Zougag, de Nebeul et de Menzel-Temim (Let.).

Cette Hélice se distingue de l'H. psammoica par sa taille moindre, par sa spire moins élevée, plus obtuse, par ses tours moins convexes et, par cela même, par sa suture moins profonde, par son ouverture plus oblique, moins ouverte, plus exactement semi-splıérique (celle de l'H. psammoica est légèrement subcomprimée à la base), par la très courte déflexion de son delnier tour à l'insertion, par sa perforation réduite à un petit point.

\section{\$ 27.}

La série des conspurcatce, qui comprend les $H$. illuviosa (Nevill.), moricola

(1) Shuttleworth, in Pfeiffer, Mon. Hel. viv. 1, 1848, p. 160 . 
(Paladilhe), polytrichia (Ancey), psara, Tenietensis, Ismirina, Arrouxi, eustricta (Bourg.), etc., est représentée, en Tunisie, par les trois formes suivantes :

I. conspureata Draparnaud, Hist. Moll. Fr. p. 105, pl. VIII, f. $33-25$, 1805 .

Daus les jardins au pied des arbustes à Kamart et à l'Ariana (Bourg. 1867); la Galite, près d'une source (Gestro, $187^{6}$ ); consulat de France à Tunis (André, $1878)$; El-Aaouina (Let.).

H. Malaspina Bourguignat, Hist. mal. Tunis, p. 14, pl. It, f. 10-14, 1868.

Sous les débris d'Opuntia au Bardo, à la Manouba; ruines d'Utique (Bourg. 1867 ); Tebourba (André, 1878 ).

I. Honorati Bourguignat, in sched. 1880 , et in Locard, Prodr. malac. Fr. p. 104 et 329,1882 .

Cette espèce, découverte en France dans l'île Saint-Honorat près Cannes, a été retrouvée en 'Tunisie, aux alentours de l'Aíaua (Bourg.) et dans la vallée de la Medjerda (Let.).

\section{$\$ 28$.}

II. Duveyrieriana Bourguignat, Valac. Alg. I, 1864 , p. 265 , pl. XIX, f. 30-35, et Moll. Sahara (Suppl. aux Touareg du Nord), p. 10, pl. XXVI, f. 6-10, 1884.

Cette Hélice, très répandıe dans la région sud de la province de Constantine, se rencoutre également dans l'oasis de Nefta (Duveyrier).

1. agrioiea Bourguignat, Malac. Alg. I, 186/, p. 201, pl. XXII, f. 1-6.

Le Iype se trouve au Djebel Meflili, dans le sud de la province de Constantine; il a été observé aussi dans une oasis au sud du Chott Faraoum (Duveyrier), où existent des échantillons bien caractérisés.

II. erisian Lelourneux et Bourguignat, sp. nov.

Testa peranguste perforita (perloratione fere punctiformi), carinata, leviter scalariformi, supra depresso-conoidali, sublus convexa, opacula, sat nitente, eleganter costata (costis in carina validioribus), uniformiter candida; spira subscalariformi, depresso-conoidea, ad summum obtusiuscula (apice minuto, rufulo); anfractibus $51 / 2$ carinatis (carina suturam sequente), convexiusculis, regulariter ac sat leute crescentibus, sutura impressula separatis; ultimo mediocri, carinato (carina ad aperturam ohsoleta), supra convexiusculo, infra convexiore, ad insertionem recto; apertura parum obliqua, lunata, transverse semioblonga; peristomate valide labiato, leviter patulo, inferne subreflexo; ad columellam parum deflexo; alt. 5 , diam. 7 millim.

Cette Hélice, des environs de Fratis (Dnveyrier), se distingue facilement des autres du même groupe par sa perforation ponctiforme, par son test fortement caréné, subscalaire-conoïdle en dessus, et par son bord péristomal fortement labié. 
Les espèces voisines de ces trois Hélices sont les H. Stolismena ${ }^{(1)}$ et Penchinati (Bourg.) d'Espagne; les H. subcostulata et Aumaliana (Bourg.) d'Algérie.

$\$ 29$.

La série des formes apiciniennes est largement développée en Tunisie. Sur les dix espèces qui composent ce groupe, six ont été constatées dans ce pays.

H. sulhapicina Mousson, in Jahrb. Malak. Ges. 1, 1874, p. 15, pl. IV, f. 2.

Échantillons bien caractérisés de cette Hélice marocaine aux alentours d'ElAouina (Let.).

H. istera Letourneux et Bourguignat, sp. nov.

Testa profunde perforata, subglobosa, solidula, opacula, sat nitente, valide striata (striis sive costulis passim crispulatis), uniformiter candidula, in prioribus circa suturam maculata; spira perobtusa convexa, ad summum quasi compressa (apice rufo, lævigato subpræminente); anfractibus 4 1/2 convexis, regulariter crescentibus, sutura (præsertim in ultimo) impressa separatis; ultimo mediocri, cylindrico, fere ex inilio perlente descendente; apertura subobliqua exigua, parum lunata, exacte splærica, peristomate recto, acuto, ad columellam vix reflexiusculo; marginibus approximatis, conniventibus; alt. 4, diam. 6 millim.

Environs de Mehedia (Let.).

Espèce remarquable par la longue direction descendante de son dernier tour, direction commençant presque à l'origine et qui, bien que lente et régulière, ne finit pas moins par devenir si accentuée que ce lour parait en contre-bas et semble dominé par la convexité de l'avant-dernier.

II. Requieni Bourguignat, in coll. 1878. (Helix apicina var. Requieni, Moquin-Tandon, Hist. Moll. Fr. Il, p. 332,1855 , et Bourg., Malac. Alg. I, 1864, p. 199, pl. XX, f. 19-20.)

Hammam-el-Lif (Berthier); Mehedia (Let.).

H. apicina Lamarck, Anim. s. vert. VI ( $2^{e}$ partie $), 1822$, p. $9^{2}, \mathrm{n}^{\circ} 102$.

De Sousa à Bir El-Buita el bords de la Medjerda près d'Utique (Gestro, 1876); El-Aouina et environs de Mehedia (Let.).

H. Mehediana Letourneux et Bourguignat, sp. nov.

Testa aperte perforata (perforatione profunda, leviter subpervia); subdepresso-globosa, subpellucide opacula, sat nitente, grosse striata quasi subcostulata, sordide albidula et flammulis translucide oleatis, irregula-

(1) Bourguignat (olim Helix vestita Rambur, in Journ. Conch. 1868 p. 267 , et 1869 p. 259 , non Helix vestita, Ferussac, in L. Pfeiffer. Mon. Hel. viv. III, p. 275, 1853).

Malacologie. 
riter dispositis, eleganter variegata; spira depressa, rotundato-convexa, perobtusa, ad summum quasi subplanulata (apice minuto, rufo, lævigato); anfractibus $41 / 2$ convexis, regulariter usque ad ultimum, deinde rapide crescentibus, sutura profunda separatis; ultimo magno, rotundato, sublus circa perforationem subturgido, ad insertionem lente subdescendente; apertura subobliqua, parum lunata, altiore quam latiore, semirotundata; peristomate recto, acuto, intus vix incrassatulo, leviter subpatulescente; columella subrecte descendente, superne rellexo-dilatata; alt. 4 , diam. 6 millim.

Environs de Meliedia (Let.).

Cette Hélice se distingue de l'H. apicina par sa perforation ombilicale moins ouverte, par sa spire plus déprimée, presque plane en dessus, par son dernier tour plus dilaté, plus volumineux, d'une taille plus élevée, moins exactement cylindrique, par son ouverture moins oblique, plus ample, plus haute que large, non aussi régulièrement semi-sphérique, par son bord columellaire plus dilaté-rélléchi.

H. Marsiana Bourguignat, in coll. 1878 , in Servain, Moll. Esp. p. 79,1880 .

Aux alentours d'El-Aouina et de Mehedia (Let.).

\section{S 30 .}

U. Incolumis Letourneux et Bourguignat, sp. nov.

Testa profunde angusteque perforata, valide carinata, depresso-discoidea, fere æque convexa supra ac subtus, subopacula cum maculis translucide oleatis, nilente, robuste striata, sive subcostulata, albidula ac zonulis subrufulis, sæpe evanidis ac sat interruptis circumornata; spira depressa, parum producta, tectiformi-conica; apice minuto, rufulo, lævigato; anfractibus 5 subplanulatis aut subconvexiusculis, lente crescentibus, sulura fere lincari separatis; ullimo mediocri, valide carinato (carina candidiore, ad aperturam minus acuta), supra convexiusculo, infra convexiore, ad iusertioneu lente descendente; apertura obliqua, leviter lunata, transverse suboblonga; peristomate albo-labiato, undique patulo, ad columellam sat dilatito-reflexo, marginibus conniventibus șat approximatis; alt. 5 , diam. 8 millim.

Alluvions de la Medjerda près du pont de la route d'Utique (Let.).

Cette Hélice ne peut être rapprochée que de l'Helix Letourneuxiana ${ }^{(1)}$, dont elle diffère par sa taille plus forte, par son test plus épais, par ses stries plus accusées, par sa carène plus aiguë, par sa perforation moins ouverte, par son dernier tour descendant, par son ouverture transversalement oblongue, etc.

L'H. incolumis appartient au groupe de l'H. intersecia, groupe si répandu dans

(1) Bourguignat, Mal. Ajg. I, 1864 , p. 195 , pl. XX, f. 33-38. 
les contrées occidentales du système européen soumises aux influences marines. Les formes les plus importantes sont les $H$. subintersecta et Pictonum (Bourg.) de France, herbarum (Servain) de France et d'Espagne, Olisippensis (Servain) du Portugal, et Letourneuxiana (Bourg.) d'Algérie et d'Espagne.

\section{$\$ 31$.}

H. Leconffei Letourneux et Bourguignat, sp. nov.

Testa angustē perforata, subgloboso-conoidea, subtus convexa, solidula, subopacula, nitente, valide costata (costis robustis, regularibus, inter se distantibus), obscure subalbidula cum zonulis rufo-cinereis (quarum una mediana subcastanea), strigis candidis, eleganter passim interruptis; spira sat producta, conico-convexa; apice exiguo, pallidiore; anfractibus 6 convexiusculis, lente crescentibus, sutura subimpressula separatis; ultimo mediocriter majore, subcompresso-rotundato, ad insertionem vix subdescendente; apertura parum obliqua, lunata, semiovata; peristomate intus labiato, recto, ad columellam subdilatato; alt. 7 , diam. 9 millim.

Celte Helice, écralement des alluvions de la Medjerda, au pont d'Utique, fait partie d'une petite série d'espèces, dont les formes semblent particulières au nord de l'Afrique; parmi elles, nous citerons la belle $H$. Flognyana (Bourg.) de Kabylie.

\section{ऽ 32.}

II. specialis Bourg. in Servain, Moll. Esp. p. $9^{5}, 1880$.

Cette espèce espagnole, abondante en Algérie, notamment dans la province d'Oran, vit en Tunisie aux environs de Menzel-bou-Zelfa (Let.) et de Carthage (Hagenm.).

L'H. specialis appartient au groupe de l'H. Reboudiana (Bourg.), dont les formes les plus importantes sont : l'H. Tarifensis (Bourg.) d'Espagne et l' $H$. Djebbarica (Bourg.) d'Algérie.

\section{$\$ 33$.}

II. Warnieriana Bourguignat, Moll. Sahara, p. 4, pl. XXVI, f. 18-20 (in Duveyrier, les Touareg du Nord), 1864 .

Cette coquille, découverte aux environs de Kriz par le savant géographe Duveyrier, a été depuis retrouvée aux alentours de Nefta (André, 1878), ainsi que dans les provinces d'Oran et de Constantine.

IH. irrita Berthier, in litt. $\mathbf{1} 880$.

Testa aperte perforata, carinata, supra tectiformi, subtus convexa, opaca, solidula, nitidissima, sublævigata quasi polita, ac sub lente acriore obsolete striatula, uniformiter candida cum zonula supera rufa et interrupta, aut aliquando candida cum zonulis inferis plus minusve numerosis; spira parum producta, tectiformi-conica; apice rufo, lævigalo, mi- 
nuto; anfractibus 5-6 subconrexiusculis, regulariter crescentibus, sutura sublineari separatis; ultimo sat majore, carinato (carina ad aperturam obsolela), supra convexiusculo, infra convexo, sat turgido, ad insertionem breviter ac subito dellexo; apertura obliqua, lunata, semirotunda; peristomate recto, acuto, intus valide labiato, ad columellam dilatatoreflexo, marginibus subcomiventibus; alt. 6, diam. 9 millim.

Ruines de Carthage el environs de Tunis (Berthier, 1880); Beja (Hagenm.); Djebel Bou-Kourneïn et alluvions de la Medjerda au pont d'Utique (Let.). Cetle espèce a encore été constatée dans le sud saharien de la province de Constantine, entre Setil el Cedraia (Jus).

L'Il. irvita diffère de l'Il. Warmieriana par sa perforation plus ouverle, par sa coquille plus déprimée, par sa spire moins conique et de moitié moius élevée, par la détlexion subile de sou dernier tour, par sa carène plus prononcée, par son péristome plus vigonrensement bordé, enfin par la forme de son ouverture, semicirculaire dans un sens transverse, paraissant plus large que haute, tandis que chez l'Il. Warmieriana, arrondie dans le sens vertical, elle semble plus haute que large.

Les différentes espèces du groupe de l'H. irrita, sans compter l'II. Warmieriana, sont l'II. Warnieropsis (Bourg. ) des Baléares, l'H. Edetanorum (Servain) d'Espagne et l'll. vaga (Pechand) d'Algérie.

\section{\$ 3 亿.}

IH. Rozetopsis Letourneux et Bourguignal, sp. nov.

Testa anguste profundeque perforala, subcarinato-angulata, depressoconica, subtus mediocriter convexa; subopacula, sat fragili, subtiliter striata (striis arclis, regularibus, mimutis, nililominus costulis similibus), albida cum zonula mediana rufa interrupta ac carinam sequente; spira sat producta, tectiformi-conica; apice minuto, lxvigato, modo ad verticis extremitaten puncliformi-atro; anfractibus 6 fere planulatis aut obscure subconvexiusculis, lente crescentibus, sutura sublineari separatis; ultimo mediocriter inajore, compresso-angulato, infra convexiore quam supra, ad insertionem recto; ipertura subobliqua, lunala, transverse semioblonga; peristomate recto, aculo, intus valide labiato, inferne leviter subpatulo, ad columellan parum dilatato; alt. 7 , diam. 10 millin.

Cap Bon (Let.).

Cette espèce se distingue de l'II. Rozeti (Michand) par sa forme plus grêle, par sa spire conique, bien tectiforme (celle de l'H. Rozeti est conique tout en affectant un contour convexe), par ses tours presque plans et non aussi renflés que ceux de l'II. Rozeti, par sa carène moins aiguë, ressemblant plutôt à une angulosité, par son dernier tour moins convexe en dessous, el notamment par son ouverture trausversalement seni-oblongue, et non arrondie.

Les Hélices de cette série sont les H. Rozeti (Michaud), Hipponensis (Morelel), 
Hipponella (Pechaud), gonia et Rokniaca (Bourg.) d'Algérie, et l'H. Meda (Porro) de Sicile.

\section{§ 35 .}

H. Hola Bourguignat, sp. nov.

Testa anguste profundeque perforata, subglobosa, subpellucida, nitida, argute striatula, sublutescenti-albidula cum zonulis 4-5 subevanidis, quasi translucide oleatis; spira convexo-subconoidea ; apice rufulo, parvo, lævigato; anfractibus 6 convexiusculis, regulariter ac sat lente crescentibus, sulura impressula separatis; ullimo majore, subrotundato, ad initium obscure subangulato, ad insertionem breviter descendente; apertura obliqua, lunata, transverse in directione declivi semiovala; peristomate rosaceo, acuto, intus robuste labiato, superne recto, inferne patulo, ad marginem columellarem robusto ac dilatalo; alt. 8, diam. 10 millim.

Environs de Nefta (Duveyrier).

Cetle espèce fait partie du groupe de l'Helix Aradasi (Mandralisca), ou autrement dit, si l'on adopte l'opinion du professeur Mousson, de celui de l'Helix turbinata de Jan (non H. turbinata des auteurs). La plupart des formes de ce groupe, telles que les H. Aradasiella, nostra (Bourg.), sont siciliennes.

\section{$\S 36$.}

II. mieromphalus Letourneux, Exc. malac. Kab. in Ann. malac. I, 1870 , p. 304.

Cetle jolie coquille algérienne a été retrouvée au Kef El-Djerdja et dans la vallée supérieure de la Iledjerda (Let.).

Cette Hélice appartient à une série assez nombreuse dont les formes sont réparties en Aloérie (H. Gerardi, Lirouxiana, Bourg., H. Russidana, Pechaud), aux Baléares (H. Arbetana, Berthier), en Sicile (H. Ophelima, Callareta, Bourg.), etc., et même jusquà Corfou (H. emasculata, Let.).

\section{$\$ 37$.}

II. misara Bourguignat, 1875, in Servain, Moll. Esp. p. 103, 188o, et Locard, Prodr. mal. Fr. p. 115 et 341,188 .

Celte forme est une espèce cosmopolite. Constatée d'abord en Sicile, puis en France, ensuite sur les bords de la Mer Noire à Sinope, elle a été retrouvée en Tunisie aux alentours de Tebourba (Pechaud).

Nous ne connaissons que deux Hélices de ce groupe, l'H. rathymia (Bourg.) de Dalmatie et l'H. mastorella (Pechaud) d'Algérie.

\section{$\S 38$.}

H. diloricata Bourguignat, sp. nov.

Testa punctiformi-perforata, angulata, supra tectiformi-convexa, subtus convexiore, subopacula, nitente, subtiliter striatula, quasi polita, uniformiter lutescente; spira convexa, tectiformi-subconoidea; apice minuto, 
obtuso, rufulo, lævigato; anfractibus 5 1/2 vix convexiusculis, fere decliviplanulatis, regulariter crescentibus, sutura lineari separatis; ultimo majore, angulato (angulo pallidiore, ad aperturam subevanescente), ad initium compresso, ad aperturam turgido-rotundato, ad insertionem recto; apertura parum obliqua, lunata, semirotundata, æque alta ac lata, intus luteola; peristomate recto, acuto, intus labiato, ad marginem columellarem in formam triangulam superne dilatato; alt. 8, diam. 11 millim.

Cetle espèce, dont le lype a été découvert en Algérie sur les remparts de Bougie, vit également en Tunisie aux environs d'El-Aouina (Let.).

On rencontre à Menzel-bou-Zelfa une variété à test plus anguleux, plus épais, moins jauıâtre, orné d'une petite bande supérieure noirâtre çà et là interrompue.

\section{u. Vafella Letourneux et Bourguignat, sp. nov.}

Testa perforata (perforatione sat aperta), angulata, supra depresso-convexa, subtus turgida ac convexiore, opacula, nitente, striatula, lutescente aut sxpius luteola cum zonulis rufulis plus minusve numerosis; spira depressa, perobtusa, mediocriter convexa; apice rufo, uitido, lævigato; anfractibus 6 vix convexiusculis, regulariter crescentibus, sutura lineari separatis; uttimo magno, ad initium compresso ac valde angulato, ad aperturam exacte rotundato, ad insertionem recto; apertura subobliqua, lunata, semispharica; peristomate recto, peracuto, intus profunde labiato, ad columellam dilatalo-reflexo; alt. 7, diam. 10 millim.

Euvirons d'Et-douina (Lel.).

Celte coguille se distingue de la précédente par sa perforation plus ouverte, par son test très dépriné, offrant en dessus une surface légèrement convexe et non subtectiforme-conoïde, par son angulosité plus accentuée et disparaissant presque subitement, tandis que celle de l'll. diloricata se prolonge jusqu'à l'ouverlure, par' son péristome plus profondément borlé, enfin par son bord columellaire très robuste, dilaté-réfléchi dans toute son étendue, non mince el seulement réfléclii triangulairement à la parlie supérieure.

Ces deux Hélices font parlie du groupe de l'II. Castroiana ${ }^{(1)}$ d'Espagne, dont les formes les plus intéressantes sont l'I. propria (Galland) de Constantinople, et les II. ademata el Avenionensis (Bourg.) du midi de la France.

\section{§ 39.}

I. IIalia Berthier, sp. nov.

Testa aperte perforata, subgloboso-depressa, fere æeque convexa supra ac infra, solida, opaca, parum nilente, sat valide striata (striis in ultimo validioribus, sæpe interruptis ac passim quasi crispulatis), subalbido-

(1) Servain, Moll. Esp. p. 74, 1880. 
cinerescente cum zonulis 6-7 atro-rufis interruptis aut sæpe inter se confluentibus; spira depressa, parum convexa; apice minuto, atro, nitidissimo ac lævigato; anfractibus 5 vix convexiusculis, lente usque ad ultimum crescentibus, sutura impressa separatis; ullimo magno, relative amplo, ad initium subcompresso, ad aperturam rotundato, ad insertionem breviter descendente; apertura obliqua, parum lunata, fere circulari, intus luteo-aurantiaca cum zonulis atris, non interruptis, apparentibus; peristomate recto, acuto, intus valide labiato, inferne patulescente, ad marginem columellarem mediocriter reflexo; alt. 10 , diam. 15 millim.

Var. $\beta$. E typo testa uniformiter cinerea cum zonula supera subevanida et spira subconoidea differt.

Le type de cette Hélice se rencontre aux environs d'Oran sur les bords de la mer. En Tunisie, la variété a été recueillie dans la partie nord des ruines de Carthage (Berthier). On trouve aussi assez fréquemment dans cetle localité des échantillons d'une taille presque de moitié plus petite que le type.

\section{I. salivosa Letourneux et Bourguignat, sp. nov.}

Testa umbilicata (umbilico profundo, parum aperto, nihilominus pervio), angulata, supra convexo-rotundata, subopacula, sat tenera, eleganter costulata (costulis strictis, productis, regularibus, in angulo validioribus), lutescente cum zonulis 5-6 atris interruptis, quarum una latior, superior circa peripheriam, et cæteræ inleriores, angustissimæ, sæpe evanidæ; spira convexa, sat producta; apice minuto, rufo, lævigato; anfractibus 6 vix convexiusculis, lente crescentibus, sutura sublineari separatis; ultimo parum majore, angulato, ad aperturam convexodeclivi, subtus convexo, ad insertionem recto; apertura obliqua, lunata, externe leviter subangulata, semirotundata, intus albido-margaritacea cum zonula atra apparente; peristomate recto, acuto, intus subrosaceolabiato, ad marginem columellarem dilatato-expanso; alt. 8, diam. 12 millim.

Sous les rochers au Djebel Reças (Let.).

II. Lallemantiana Bourguignat, Mal. Alg. 1, 1864, p. 211, pl, XXI, f. 31-35.

Le type n'a pas été rencontré en Tunisie, mais on y trouve une variété d'une taille plus petite, à dernier tour assez anguleux : Kef El-Djerdja (Let.).

Ces trois Hélices, avec l'H. Tripieri (Pechaud) d'Algérie et l'H. Chalepia (Bourg.) de Sicile, composent le groupe de l'H. Lallemantiana, groupe très reconnaissable à ses espèces caractérisées par une surface supérieure convexe en forme de dôme, par un test sillonné de fortes striations serrées, saillantes et toujours régulières.

II. Cretica Ferussac, Prodr, $n^{\circ} 288$, et L. Pfeiffer, Symb. Hel. I, 1841, p. 40, et ( $2^{\circ}$ édit. 
Chemnitz) Helix, p. 253 , pl. XXXVII, f. 21 -a a (médiocres), et Mon. Hel. viv. I, 1847 , p. 159 , Bourg. Mal. Alg. I, 1864 , p. 232 , pl. XXV, f. $16-18$ (exactes).

Cette Hélice, abondante dans les îles de l'Archipel, a été importée dans le nord de l'Afrique où elle se trouve çà et là bien caractérisée : environs de Tunis (Bourgr. 1867), Ä̈n-ben-Khelil (sud de la province d'Oran), territoire des Aït Ziki (Djurdjura).

Kobelt et quelques auteurs ont signalé sous ce nom des formes algériennes qui nont pas le moindre rapport avec elle.

\section{$\$ 41$.}

1. astata Bourg. in Servain, Moll. Esp. p. $110,1880$.

Cette magnifique espèce, découverte anx alentours de Tunis (Bourg. 1867), a été retrouvée en Alyórie, à Boufarik, à Tablat entre Alger et Aumale, ainsi qu'aux environs d'Alger; depuis, elle a été constatée près de Valence en Espagne, et à Vence dans les Alpes-Maritimes.

\section{s 42.}

r. Menzelensis Letourneux et Bourguignat, sp. nov.

Testa perforata (perforalione superficiali, modo in ultimo aperta), subgloboso-depressa, opaca, crelacea, strialuła, nitida, uniformiler candida; spira subconoidali, sicut in ullimo transverse amplissimo apposita; apice atro, minuto, obtuso ac lævigato; anfraclibus 6 parum convexiusculis, perlente usque ad ullimum crescentibus, sutura impressa separatis; ultimo inaximo, transverse amplissimo, subrotundato, subtus leviler quasi compresso-subplanulato, ad insertionem recto; apertura parum obliqua, lullala, trausverse semiovata, inlus luleola; peristomale recto, aculo, intus profunde bimarginato, ad marginem columellarem dilatato-reflexo; alt. 12 , dian. 19 millim.

Environs de Menzel-Temim (Let.).

Cette coquille est remarquable par un dernier tour si amplement développé que la spire semble toule exiguë et comme juxtaposée.

H. talepora Bourguignat in Locard, Prodr. mal. Fr. p. $9^{8}$ et 325, 1882.

Bords de l'Oued Zergua (Hagenmüller).

\section{S 43.}

H. membroniea Berthier, sp. nov., 1880 .

Testa profunde abrupteque umbilicata, angustata, supra depressa, vix convexa, perobtusa, infra convexiore, opacula, nitente, valide striata aut aliquando leviter striatula, uniformiter candida aut albida cum zonulis subrufulis, subevanidis, interruptis et variegatis; spira depressa, parum convexa; apice obtuso, rufo, lævigato; anfractibus $51 / 2$, supra vix con- 
vexiusculis, lente crescentibus, sutura subimpressula aut sublineari separatis; ultimo mediocriter majore, angulato (angulo superiore, ad aperturam evanescente), supra convexiusculo, infra convexo, circa umbilicum turgido et obscure subangulato, superne ad insertionem recto; apertura obliqua, lunata, semirotundata; peristomate recto, acuto, intus uni- aut bimarginato, ad colunetlam crassiore ac leviter dilatato; alt. 5 1/2, diam. $81 / 2$ millim.

El-Aouja près Porto-Farina (Berthier).

H. artonilla Hagenmüller, sp. nov., 1880, citée par Locard, Prodr. mał. Fr. p. 340 , 188з.

Testa profunde, pervie lateque umbilicata (umbilico aperto, in ultimo sat dilatato), angulata, supra depresso-tectiformi, subtus convexa, opacula, nitente, striatula, sæpe quasi polita, albida cum zonulis subrufis vix perspicuis; spira parum convexa, tectiformi, perobtusa; apice minuto, rufo ac lævigato; anfractibus 6 declivi-subplanulatis, lente usque ad ultimum crescentibus, sutura lineari, in ultimo impressula, separatis; ultimo majore, angulato (angulo obsoleto, superiore, ad aperturam evanescente), supra convexiusculo, infra rotundato-convexo et circa umbilicum turgido, superne ad insertionem recto; apertura obliqua, lunata, semiovato-rolundata; peristomate recto, acuto, intus robuste labiato, inferne leviter patulescente ac ad columellam crasso et sat dilatato; alt. 7 , diam. 13 millim.

Cette espèce, découverte par le docteur Hagenmüller, le long de la ronte de Bône à la Calle, a été constatée en Tunisie au Cap Negro et aux environs de Bizerte (Berthier).

Elle se distingue de la précédente par sa taille plus grande, par sa spire moins déprimée, par son angulosité moins pronoucée, par son dernier tour plus développé, par son péristome plus fortement bordé et légèrement patulescent à la base, par son ouverture semi-arrondie-ovalaire dans une direction légèrement transverse, enfin notamment par son ombilic en forme d'entonnoir largement ouvert et laissant voir l'enroulement spiral interne. Chez l'H. membronica, l'ombilic, non évasé, ressemblant à un trou profond à pic, ne laisse apercevoir aucun des tours.

H. astonara Hagenmüller, sp. nov., 1880, citée par Locard, Prodr. mal. Fr. 188a, p. 340 .

Testa profunde umbilicata (umbilico parum aperto ac pervio), angulata, depressa, supra convexiuscula, infra convexa, solida, opacula, cretacea, nitente, striatula, candida cum zonulis rix perspicuis; spira leviter convexa, subtectiformi; apice exiguo, rufulo, nitidissimo; anfractibus 6 vix convexiusculis lente usque ad ultimum crescentibus, sutura impressula separatis; ultimo majore, angulato (angulo superiore, plus minusve acuto, ad aperturam evanido), supra convexiusculo, infra de- 
clivi-convexo usque ad angulum circumumbilicalem, superne ad insertionem recto; apertura parum obliqua, lunata, transverse semiovata; peristomate recto, aculo, intus profunde robusteque labiato, ad columellam crasso et parum dilatato; alt. 7, diam. 13 millim.

Euvirons de Bizrtte (Berthier). Le lypr se trouve entre Bòne et la Calle (Hagenmüller).

Cette coquille diffère de la précédente par son test plus crétacé, par son angulosilé plus prononcée. par sa spire moius régulièrement tectiforme, par son dornier tour moins déclive à sa partie supérieure et plus renflé-anguleux en dessous nutour de l'ombilic, par son ouverture plus ovalaire dans le sens transversal, par son bord péristomal plus épais, plus fortement bordé, et par son ombilic non évasé, plus étroit cl laissant à peine voir l'enroulement du dernier tour.

Ces trois espèces font partie d'un groupe auquel appartienncnt encore l'II. Dolmenica (Bourg. d'Algérie et l'H. Patrasi (Letourneux) de Grèce.

\section{$\$ 44$.}

II. noctuella Bourguignat, sp. nov.

Testa profunde ac modice pervie umbilicata, depressa, supra vix convexa, infra convexiore, angulata, solida, cretacea, opaca, nitente, costulata (costulis inferioribus tenuioribus), albidula cum zonulis rufulis perinterruptis, vix perspicuis; spira parum compressa ; apice minuto, rufo, levigato; anfractibus 5 convexiusculis, regulariler crescentibus, sulura impressula separatis; ultimo majore, angulato (angulo superiore, sat compresso, ad aperturan subevanescente), supra convexiusculo, infra convexo ac circa unbilicum turgido, superne ad insertionem breviter descendente; apertura parum obliqua ac lunata, semirotundata, bimarginata; peristomale crasso, nihilominus recto, acuto, intus valide albo-labiato, ad columellam perrobustam dilatato; alt. 6, diam. 10 millim.

Coteaux arides d'Oudena entre Mahamedia el le Djebel Reças (Bourg. 1867); colline à Beja (Hagenınüller).

Celtc forme appartient à la séric de l'II. Romulina (Servain) d'Espagne, dont les principales espèces sont l'H. Tarovana (Calcara) de Sicilc, l'H. Egeana (Bourg.) de lî̀le d'Eubée, lH. Corcyrana (Let.) de Corfou, etc. Parmi ces Hélices, la plus voisine est l'H. Romulina, dont elle diffère notamment par son ombilic plus ouvert du double, par son ouverture moins grande, par la direction descendante de son dernier tour, ctc.

\section{$\$ 45$.}

H. pleurabdota Bourguignat, sp. nov., 1880 .

Testa profunde angusteque umbilicata, depresso-subglobosa, solida, cretacea, nitente, valide costulata, candida ac zonulis rufo-castaneis, albointerruptis (quarum superior lata, ad suturam pallescens, inferiores 
2 vel 3 ) circumcincta; spira sat producta, subconoidali-convexa, ad summum obtusa; apice minuto, lævigato, rufulo; anfractibus 6 convexiusculis, sat lente ac regulariter crescentibus, sutura impressula separatis; ultimo majore, rotundato, ad insertionem descendente; apertura parum obliqua, mediocrifer lunata, subsphærica, intus aurantiaca; peristomate recto, acuto, intus valde labiato, ad columellam robustam superne leviter dilatato; marginibus sat approximatis; alt. 9, diam. 13 millim.

Revers des fossés du Bardo près Tunis (Bourg. 1867); environs de Beja (Hagenm.).

\section{II. cacista Bourguignat, sp. nov., 1880 .}

Testa profunde angusteque umbilicata, subgloboso-conoidali, semper deformata, quasi streptaxiformi, cretacea, solida, opaca, costulata, albidula aut albido-lutescente cum zonulis rufulis, angustis, interruptis ac plus minusve pallidioribus; spira sat elata, subconica, leviter streptaxidea; apice minuto, nitido, lævigato; anfractibus 6-7 convexiusculis, regulariter usque ad ultimum crescentibus, sutura impressula separatis; ultimo majore, ad aperturam sat amplo, rotundato, ad initium aliquando leviter angulato, superne ad insertionem descendente; apertura obliqua, parum lunata, semioblonga in directione declivi-transversa, intus sæpe rubella; peristomate recto, acuto, valide labiato, ad columellam dilatato, marginibus approximatis; alt. 10 , diam. 15 millim.

Cette espèce, remarquable par sa forme streptaxoïde se rencontre dans les lieux arides sur le bord des chemins aux environs de Tunis (Bourg. 1867).

\section{I. vaganensis Hagenmüller, sp. nov., 1882 .}

Testa profunde et anguste umbilicata, depresso-globosa, supra modice convexa, infra tumidiore, opacula, subcretacea, nitente, valide costulata, sordide albidula cum zonulis rufulis perinterruptis ac sæpe vix perspicuis aut maculas irregulariter sparsas simulantibus; spira parum convexa, in tholum rotundata; apice minuto, rufulo, nitido ac lævigato; anfractibus 6 convexiusculis, regulariter crescentibus, sutura impressula separatis; ultimo magno, turgido-rotundato, superne ad insertionem recto aut vix descendente; apertura mediocriter obliqua, lunata, semirotundata, intus rubro-aurantiaca; peristomate recto, acuto, intus valide labiato, ad columellam robustam leviter dilatato, marginibus sat remotis; alt. 10, diam. 15 millim.

Var. minor. Alt. 7 1/2, diam. 12 millim.

Alentours de Beja (Hagenm.) et de Tunis dans les endroits brûlés par le soleil (Bourg. 1867). 
II. syntela Bourguignat, sp. nov,, 1880 .

Testa profunde ac leviler pervie unibilicata, depressa, utrinque fere æqualiter convexa, opacula, nitente, subtiliter costulata, lutea ac zonulis stramineis numerosis et flammulis ejusdem coloris circa sutnram inter se aqualiter sparsis eleganter ornata; spira modice convexa, ad summum obtusa; apice rufulo, exiguo; an fractibus 6 convexiusculis, regulariter crescentibus, sutura impressula separatis; ultimo majore, ad initium leviter compresso, ad aperturam subturgido, superne ad insertionem recto; apertura subobliqua, sat ampla, parum lunata, subrotundata, intus alboluteola; peristomate recto, acuto, intus profunde labiato, ad columellam dilatato; alt. 8 , diam. 12 millim.

Resers des fossés de ta Manouba el du Bardo (Bourg. 1867); colline à Beja (Hagenmüller).

\section{H. perlutosa Hagemmüller, sp. nov., 1881 .}

Testa profunde angusleque umbilicata, depressa, leviter subangulata, parum opacula potius subtenera, nitente, sat valide costulata, griseo-luteola cum zonulis vix perspicuis, valde interruptis; spira leviter convexa; apice minuto, rufo, nitido ac levigato; infractibus 6 subconvexiusculis, regulariter crescentibus, sutura subimpressula, in ultimo magis impressa, separatis; ultimo mediocriter majore, subrotundato-compresso, angulato (angulo ad aperturam parum impresso), superne e dimidio evolutionis lente descendente; apertura perobliqua, parum lunata, semioblonga in directione declivi, intus luteo-margaritacea; peristomate recto, aculo, intus labiato, ad columellanı crassiore el mediocriler dilatato, marginibus silt approximatis; alı. 7, diam. $11 \mathrm{millim}$.

Cette espèce, remarquable par sa forme déprimée et par la longue descente de son dernier tour, a été trouvée aux alenlours de Tebourba (Hagenmüller).

Ces cinq Hélices sont pour la Tunisie ce que sont pour le Maroc les H. Jaylei (Paladilhe) rusticella, mona el doryphora (Pechaud).

\section{\& 16.}

H. Parthenia Hagenmüller, sp. nov.

Testa profunde subpervieque umbilicata, depresso-subglobosa, fere æqualiter utrinque convexa, sat tenera, subpellucida, nitente, valide striata quasi costulata, albido-luteola cum maculis rufis in zomulas interruplas dispositis; spira mediocriter convexa, ad summum obtusa; apice minutissimo, rufulo, lævigato; anfractibus 6 convexiusculis, regulariter crescentibus, sulura impressula separatis; ultimo magno, ad initium compresso ac subangulato, ad aperturam amplo, rotundato, ad insertionem recto; apertura parum obliqua ac lunata, ampla, semisplıærica, intus 
luteo-aurantiaca; peristomate recto, acuto, intus labiato, inferne leviter patulo, ad columellam parum dilatato; alt. 7, diam. 11 millim.

Colline à Beja (Hagenmüller).

Cette espèce appartient à ce groupe des H. Cisalpina (P. Fagot) dont les formes nombreuses sont si répandues dans les régions circumméditerranéennes. Celles qui ont le plus de rapport avec l'H. Parthenia sont l'H. arcuata (Ziegler) de Corfou, 1'H. Ionica (Mousson) des îles Ioniennes, l'H. Lepantica (Let.) de Grèce, etc.

\section{$\$ 47$.}

H. submeridionalis Bourguignat, Mal. Alg. I, 1864 , p. 214 , pl. XXII, f. $26-29$, et pl. XXIV, f. 1-11.

Environs de Beja (Hagenmüller).

Celte Hélice, assez commune en Algérie (Alger, Djelfa, Ouled Djebara, LallaMaghnia, etc.), est une forme très caractérisée qui a été confondue, par la plupart des auteurs, avec un grand nombre d'espèces qui ont peu de rapport avec elle.

\section{Issea Hagenmüller, sp. nov.}

Testa profunde ac sat aperte perforata (perforatione abrupta, nihilominus teviter pervia), subgloboso-conoidali, subopacula, nitente, subtiliter striatuia (striis retusis, regularibus), luteolo-albidula cum zonulis 6-7 castaneis; spira sat elata, subconoidali, ad summum obtusa; apice minutissimo, nitido, lævigato ac rufulo; anfractibus 6 convexiusculis, lente usque ad ultimum, deinde rapidius crescentibus, sutura impressula separatis; ultimo majore, fere exacte cylindrico, ad insertionem descendente; apertura parum obliqua, lunata, semicirculari, intus luteola; peristomate recto, intus valide crasso-labiato, leviter patulescente, ad columellam superne breviter dilatato; alt. 9 , diam. 14 millim.

Colline à Beja (Hagenmüller).

Cette espèce se distingue de la précédente par son test très finement strié presque poli et assez brillant, par sa perforation plus ouverte, par son dernier tour plus cylindrique, moins descendant à l'ouverture, par ses tours plus convexes, par sa suture moins superficielle, enfin par son ouverture plus exactement semicirculaire.

\section{I. irana Hagenmüller, sp. nov.}

Testa profunde perforata (perforatione non pervia, sed in ultimo solum leviter dilatata), depresso-globosa, opacula, nitente, striatula (striis ut in H. Iscea), albida cum zonulis numerosis, plus minusve subrufulis; spira convexa, mediocriter producta, perobtusa; apice minuto, lævigato, nitido, anfractibus 6 subconvexiusculis, lente usque ad ultimum, deinde rapide crescentibus, sutura fere lineari, in ultimo impressula, separatis; ultimo maximo, ad initium subcompresso-rotundato, ad aperturam turgido-cylindrico, ad insertionem breviter descendente; apertura subobliqua, ampla, 
lunata, semicirculari, intus albido-margaritacea; peristomate recto, intus labiato, ad columellam sat valide dilatato; alt. 10 , diam. 14 millim.

Environs de Beja (Hagenmüller).

Cette coquille, bien que d'une taille plus haute que la précédente, paraît néanuoins fort déprimée, en raison du grand développement du dernier tour, qui par sa grosseur occupe une partie de la hauleur.

L'II. Irana se distingue encore de l'H. Iscea par son ombilic dilaté senlement au dernier tour, par sa spire moins conique, par sa suture moins accentuée, par ses tours moins cylindriques, par son ouverture bien plus ample et à bord péristomal moins fortement bordé, etc.

De plus, la direction descendante du dernier tour est de moitié plus courte que celle de l'H. Isara.

\section{$\$ 48$.}

1. Mauritaniea Bourguignat in Servain, Moll. Esp. p. $107,1880$.

Cette belle Hélice, si commune dans certaines localités de l'Algérie (Tlemcen, Nansourah, Perrégaux, Mascara, etc.) a été retrouvée bien typique aux environs de Tunis (Bourg. 1867), près de l'amphithéâtre d'El-Djem (Gestro, 1876), ainsi qu’entre Sousa el Mlonastir et dans la région des Hamada (Let.).

\section{II. taria Bourguignat, sp. nov.}

Testa pervie profundeque umbilicata, conica, subopacula, nitida, striatula, in ultimo malleata, albidula et eleganter rufo-variegata cum zonulis rufis numerosis perinterruptis, ad aperturam rubella; spira elata, conica, ad summum obtusiuscula; apice minuto, rufo, tævigato; anfractibus 6 vix convexiusculis, regulariter crescentibus, sutura impressula, in ultimo magis impressa, separatis; ultimo majore, rotundato, ad initium obscure subangulato, superne ad insertionem leviter descendente; aperiura obliqua, parum lunata, semisphærica, intus mbello-iurantiaca; peristomate recto, acuto, intus aurantiaco-labiato, inferne patulo, ad columellam dilatato; alt. 10 , diam. 12 millim.

Le long des haies el sous les Opuntia au village de Sidi-Bou-Saïd (Bourg. 1867); citernes de Carthage (Hagenmüller).

Le type de celte espèce se trouve en Algérie au Hammam des Ouled Ali près Guelma (Let.).

\section{S 49.}

II. variabilis Draparnaud, Tabl. Moll. p. 73,1801 , et Hist. Moll. p. 84, pl. V, f. 11-12, 1805 .

Cetle Hélice, sous le nom de laquelle les auteurs onl fait de si grandes confusions, se rencontre bien caractérisée aux alentours de Tunis (Bourg. ${ }^{1867}$ ) el à Menzel-Temim, ainsi que sur les dunes de Nebeul (Let.). 
II. privata Galland, sp. nov., 1879 .

Testa perforata, globosa, opacula, parum nitente, argute striatula, quasi polita, uniformiter albido-subcærulea aut candidula, ad peristoma subrubello-luteola, ac sæpe in ultimo zonula unica rufa aut zonulis fere evanidis ornata; spira perobtusa, rotundato-convexa; apice rufo, exiguo, nitido ac lævigato; anfractibus 6 convexiusculis, lente usque ad ultimum crescentibus, sutura impressula separatis; ultimo magno, amplo, rotundato, superne lente descendente; apertura leviter obliqua, lunata, semiovata, intus luteo-vinosa; peristomate recto, acuto, intus labiato, ad columellam superne mediocriter dilatato; alt. 15 , diam. 21 millim.

Cette forme, qui parait abondante en Turquie, notamment aux environs de Constantinople, existe en Tunisie près de Bizerte (Berthier), ainsi qu'en Algérie aux alentours de Bône et de Souk-Ahras.

H. salentina $H$. Blanc, sp. nov., 1879 .

Cette Hélice, qui doit être décrite prochainement par le malacologiste Locard, est une espèce assez cosmopolite puisqu'elle a été constatée en Italie, en Grèce, en France, en Espagne et en Algérie. Dans la Régence, elle a été trouvée dans le territoire des Houchteta, sur la frontière entre la Calle et le Cap Roux, ainsi que dans la vallée de l'Oued Zergua (Hagenmülter).

\section{H. Zerguana Hagenmüller, sp. nov.}

Testa perforata (perforatione parum aperta), depressa, supra parum convexa, opaca, nitente, subtiliter striatula, uniformiter albida ; spira depressa, mediocriter convexa; apice sat præminente, minuto, rufo, nitido et lærigato; anfractibus 6 convexiusculis, regulariter usque ad ultimum, ac deinde celeriter crescentibus, sutura impressula separatis; ultimo magno, globoso-rotundato, turgido, ad insertionem lente ac breviter descendente; apertura paululum obliqua, lunata, subovato-semirotundata, intus luteola; peristomate recto, acuto, vix intus incrassatulo, ad columellam dilatato; alt. 13 , diam. 20 millim.

\section{Vallée de l'Oued Zergua (Hagenmüller).}

Cette espèce se distingue de la précédente par sa spire écrasée, peu convexe, et non conoïde, par sa croissance spirale plus rapide, par son dernier tour plus gros, plus ventru, plus développé dans le sens transversal et pourvu d'une direction descendante plus courte et moins prononcée, par son ouverture plus ample, presque ovale-arrondie dans le sens horizontal (celle de l'H. Salentina, moins développée, est arrondie dans une direction plus ou moins déclive, ce qui lui donne une plysionomie toute différente). 
1. messapia $H$. Blane, sp. nov., 1879 .

Testa pervie late aperieque umbilicata, subconoidali (anfractibus cylindricis, non tumidis sed tantum rotundatis), opacula, nitente, striatula, uniformiler albida, ad peristoma fusco-rubella; spira sat convexo-producta, subconoidali; apice acutiusculo, nigro, nitido et lævissimo; anfractibus 7 convexiusculis, lente crescentibus, sutura impressa separatis; ultimo mediocri, cylindrico, superne lenie descendente; apertura obliqua, parum lunala (marginibus sat approximatis), semisplærica in directione declivi, intus luteo-rubella; peristomate recto, acuto, intus vix incrassalulo, ad colun ellam dilatato; alt. 11 1/2, diam. 17 millim.

Le type se trouve en Ilalie, à Otrante. En Tunisie, cetle espèce est représentée, aux environs de 'T'unis (Berthier), par une variété que caractérise un ombilic moins ouvert.

Cette Iélice apparlient à un groupe d'espèces siciliennes, dont les principales sont l'H. pliila (Bomrg.) de Sicile et l'H. camira (Bourg.) de Sicile et d'Algérie.

\section{S 52.}

I. herbatiea Fagot, Diagn. esp. nouv. Fr. p. 13, 1884.

Sidi-Bou-Saïd près Carthage (Bourg. 1867); colline à Beja (Hagenm.).

Le type de cetle espèce se trowve en France dans la Haute-Garonne.

II. Krizensis Bourguignat, sp. nov., 1881, cilée par Locard, Prodr. mal. Fr. p. 340, 188 s.

Testa profunde perforata, conoidea, subopacula, fere semper inquinata, striatula, albidula cum zonulis subrufulis interiuptis vel fere omnino evanidis; spira elata, conoidea; apice subacutiusculo, nitido, rufo, lævissimo; anfractibus $61 / 2$ convexiusculis, lente usque ad ultimum, deinde rapidius crescentibus, sulura impressula separatis; ultimo majore, ad initium quasi obscure subangulato, subrotundato, ad insertionem recto; apertura obliqua, lunata, semiovata in directione leviter declivi; peristomate vinoso, recto, acuto, intus mediocriter labiato, ad columellam dilatato; alt. 11 , diam. 15 millim.

Kriz (Duveyrier); Tozer (Let.).

Cette forme existe aussi en Algérie, en France, en Italie et en Grèce.

H. mendranoi Servain, Moll. Esp. p. 105, 1880 .

Ruines de Carthage (Bourg. 1867); vallée de l'Oued Meliana au sud-est de Tunis (Hagenmüller). 
Cette Hélice espagnole a encore été constatée en France, en Sicile et en Algérie (le long de la route de Bône à la Galle et dans la vallée de l'Oued Kermich).

H. Canovasiana Servain, Moll. Esp. p. 104, 1880.

Le type n'a pas encore été trouvé dans la Régence, mais simplement une variété minor aux alentours de Tebourba (Pechaud).

L'H. Canovasiana est une forme hispanique qui a été, ces années dernières, constatée en France, en Sicile et en Algérie.

II. Solanoi Servain, Moll. Esp. p. 96, 1880 .

Environs de Tebourba (Pechaud).

Cette Hélice espagnole a été signalée dernièrement sur les bords de la route d'Alger à Mustapha.

H. alluvionum Servain, Moll. Esp. p. $102,1880$.

Variété à ombilic plus ouvert, sur les ruines de Carthage (Berthier).

Le type de cette coquille se trouve à Pampelune (Espagne). Cette Helice a encore été constatée en France et en Algérie dans les alluvions de la Seybouse près Guelma et de l'Oued Sebaou près Dellys.

H. tremata Letourneux et Bourguignat, sp. nov.

Testa profunde perforala, subglobosa, supra subconica, infra conrexa, subopacula, nitente, striatula, uniformiter al bida; spira sat productoconica; apice rufulo, nitido, lævigato; anfractibus 6 convexiusculis, lente usque ad ultimum, deinde rapidius crescentibus, sutura impressula separatis; ultimo magno, ad initium subangulato, ad aperturam exacte cylindrico, ad insertionem recto; apertura vix obliqua, sal lunata (margines remoli), semicirculari, æque alta ac lata; peristomate vinoso, acuto, nihilominus crasso-labiato, leviter patulescente; columelta parum dilatata, sat recte descendente; alt. 8 , diam. 11 millim.

Environs de Tozer (Duveyrier et Let.).

Ces sept Hélices font partie d'une série d'espèces très nombreuses répandues dans presque toutes les contrées du pourtour de la Méditerranée. Toutes ont le péristome rouge ou vineux.

\section{S53.}

H. Tritonidis Jus, sp. nov. 1883 , et Coq. terr. vivant dans le Sahara du département de Constantine, 1883.

Testa punctiforme profundeque perforata, parvula, obscure angulata, supra conoidea, subtus convexa, opaca, nitente, subtiliter striatula, candida; spira sat convexo-conica, ad summum obtusa; apice rufo, nitido, lævigato; anfractibus $51 / 2$ vix convexiusculis, lente nihilominus in ultimo magis crescentibus, sutura fere lineari separatis; ultimo mediocriter majore, angulato fere usque ad peristoma, supra convexo, infra convexiore, 
ad aperturam rotundato, ad insertionem recto; apertura subobliqua, lunata, semirotundata, intus candida; peristomate lúteo-stramineo, recto, intus valide labiato, leviter subpatulescente, ad columellam parum dilatato; alt. $51 / 2$, diam. 8-10 millim.

Le type se trouve entre Cedraiat et Setil au sud de Biskra, dans le Sahara de la province de Constantine.

Dans la Régence, cette espèce a été trouvée anx environs de Gabès (Let.) et de Tebourba (Pechaud).

I. fera Bourguignat, sp. nov.

Testa punctiformi-perforata, parvula, depressa, angulata, fere sque convexa supra ac infra, subopacula, parum nitente, striatula, albidula; spira convexo-subtectiformi, parum producta, ad summum perobIusa; apice atro, nitidissimo; anfractibus 5 vix convexiusculis, regulariter ac sat lente crescentibus, sutura parum impressula separatis; ultimo sat majore, angulato (angulus ad aperturam evanescens), ad initium compresso, ad peristona subrotundato, ad insertionem recto; apertura subobliqui, lunata, semirotundato-ovala in directionem declivem, intus luteola; peristomate rubello, recto, plus minusve labiato, ad columetlam ditatato; alt. 6 , diam. 9 millim.

Cette forme, plus déprimée et à ouverture subovalaire-arrondie dans une direction déclive, a été déconverte aux environs de Tebourba (Berthier), ainsi que dans les antiques citernes de Carthage (Hagenmüller). Elle a été constatée dernièrement en France, aux Issards, dans les Bouches-du-Rhône (Locard).

Ces deux Hélices font partie du groupe de l'H. pseudenhalia (Bourg.) du château d'If, près Marseille ${ }^{(1)}$, dont les plus imporlantes espèces sontl'H. subneglecta (Bourg. 1860) du château d'If, de Bòne et d'Alger; l'H. Megarridensis (Bourgog. 1877 ) de Mégarride en Grèce et de Sinope en Anatolie; l'H. nova (Bourg. in Servain, Moll. Esp. p. 103, 1880. Helix Hellenica [non Bourg. Sp. noviss. $\mathrm{n}^{\circ}$ 62, $187^{\circ}$ ] Westerlund et Blanc, Mal. Grèce, p. 70, 1879) d'Athènes et de l'ile d'Eubée; l'H. Blasi (Servain, 1880) de Badajoz en Espagne, de Bône en Algérie, de la Barre-de-Mont en Vendée et des environs de Toulon; l'H. Octavia (Let. 1884) de Tenez en Algérie; l'Il. Marsillionensis (Coutagne) des Bouches-du-Rhıne; l'H. Hellenica [non Westerlund et Blanc] (Bourg. 1876) de Grèce, etc.

\section{S 54.}

I. Casertana Bourguignat, sp. nov. 1875 .

Testa punctiformi-perforata, parvula, subconica, sat tenera, subpellucida, argutissime striatula, albido-cinerea, ad peristoma rubella, ac zonulis

(1) Cette coyuille a été également constatée à Menton dans les Alpes-Marilimes et à Tlemcen en Algérie. 
numerosis, fere evanidis aut subtranslucidis, vel maculis rufulis in series dispositis, ornata; spira sat producta, subconica, ad summum obtusa (apex exiguus, lævigatus); anfractibus 5 convexiusculis, regulariter crescentibus, sutura vix impressula separatis; ultimo majore, rotundato, ad insertionem plus minusve descendente; apertura obliqua, parum lunata, fere semicirculari; peristomate recto, luteo-rubello, intus labiato, inferne subpatulescente, ad columellam dilatato et reflexo; alt. 6, diam. 9 millim.

Var. major. Testa major (alt. 8, diam. 10 millim.) et crassior.

Le type, ainsi que son nom l'indique, a été trouvé à Caserte, près de Naples, dans les jardins du palais.

En Tunisie, la variété major, seule, a été constatée à Tebourba (Berthier) et à l'île de Tabarka (Let).

II. Meindozat Seroain, sp. nor. 1880 , et Locard, Prodr. Malac. Fr. p. 115 et 343 , 1888.

Environs de Tebourba (Berthier).

C'est à la Barre-de-Mont, en Vendée, que cette Hélice a été signalée pour la première fois par le docteur G. Servain. Depuis elle a été découverte dans la presqu'ile de Rhuiz (Morbihan) près du château de Sucinio.

I. Ogiaca Servain, sp. nov. 1880 , in Locard, Prodr. Mal. Fr. p. 115 et $343,1882$.

Cette forme de l'île d'Yeu, sur les côtes de la Vendée, a été retrouvée bien caractérisée aux alentours de Gabès (Let.).

\section{H. Tacapica Letourneux et Bourguignat, sp. nov.}

Testa punctiformi-perforata, globoso-conoidali, subopacula, nitente, subtilissime striatula, albidula, ad peristoma lutea aut rubella, ac zonulis subtranslucide rufulis, passim flammulatis, eleganter cincta; spira producta, conico-convexa, ad summum obtusa; apice minutissimo; anfractibus 6 convexiusculis, regulariter crescentibus, sutura impressula separatis; ultimo majore, ventroso-rotundato, ad insertionem recto; apertura subobliqua, lunata, hemisphærica, intus luteo-albida, cum zonulis apparentibus; peristomate recto, valide labiato ac subpatulescente, ad columellam robustam valde dilatato; alt. 9 , diam. 12 millim.

Environs de Gabès (Let.).

Aux alentours de l'Oued Cherchara (Let.), on rencontre une forme minor (H. 7 , D. 9 mill.), à bandes plus larges et au dernier tour légèrement descendant à linnsertion.

n. Tabarkana Letourneux et Bourguignat, sp. nov.

Testa punctiformi-perforata, parvula, globosa, conoidea fere æque alta ac lata, sat tenera, subpellucida, argute siriatula, albido-luteola, ad 
peristoma rubra et zonulis rufis passim variegatis eleganter ornata; spira producta, conica, ad summum obtusa (apex minutus, nitidissimus, rufulus ac lævigatus); anfractibus 6 vix convexiusculis, regulariter crescentibus, sutura fere lineari, in ultimo solum impressula, separatis; ultimo majore, turgido, cylindrico, ad insertionem descendente; apertura parum obliqua ac lunata, semicirculari, intus luteo-rubella cum zonulis apparentibus; peristomate rubro, recto, acuto, intus profunde leviter labiato, ad columellam relative valde dilatato ac supra perforationem leviter reflexo; alt. $81 / 2$, diam. 9 millim.

lie de Tabarka (Let.).

\section{W. ana Letourneux et Bourguignat, sp. nov.}

Testa profunde ac sat aperte perforata, conoidea, opacula, 'sólida, parum nitente, argute striatula, sordide albidula cum zonulis vix perspicuis translucide oleatis; spira producta, conica, ad summum mediocriter obtusa; anfractibus 6 convexiusculis, lente usque ad ultimum, deinde velociter, crescentibus, sutura impressula, in ultimo impressiore, separatis; ultimo maximo, ad initium sicut subcompresso, ad aperturam rotundato, superne recto, sicut subascendente ac in penultimo ventrem leviter faciente; apertura obliqua, ampla, parum lunata (margines approximati), fere circulari, intus albo-margaritacea; peristomate recto, robusto, valide labiato, inferne patulo, ad columellam dilatato; alt." $\mathbf{9}$, "diam. 12 millin.

Environs de Tozer et de Gabès (Let.).

\section{Tebourbana Letourneux et Bourguignat, sp. nov.}

Testa anguste perforata, conica, subopacula, nitente, striatula, pallide luteola; spira producto-conica, ad summum acutiuscula (apex ater, nitidissimus); anfractibus 6 subconvexinsculis, lente usque ad ultimum, deinde rapidius crescentibus, sutura inpressula, in ultimo impressiore, separatis; ultimo majore, ad initium angulato (angulus fere subito evanescens), ad aperturam anplo, turgido-cotundato, ad insertionem recto; apertura obliqua, lunata, semirotundata, intus albida; peristomate recto, aculo, intus profunde mediocriter labiato, ad columellam sat ditatato; alt. 9, diam. 11 miltim.

Environs de Tebourba et de Gabès (Let.).

Celte espèce se distingue de la précédente par son test plus mince, par sa perforation plus étroite et moins profonde, par son dernier tour anguleux à son origine et ne faisant pas saillie, à sa partie supérieure, sur l'avant-dernier, par són bord péristomal mince, profondément labié, non patulescent à la base et infiniment moins épais, etc. 
H. therella Berthier, sp. nov.

Testa profunde aperteque perforata, conica, opacula, striatula, candida; spira producta, conica, ad summum obtusiuscula (apex rufulus, minutissimus); anfractibus 6 subconvexiusculis , lente usque ad ultimum, deinde velociter crescentibus, sutura vix impressula, in ultimo impressiore, separatis; ultimo magno, rotundate, ad aperturam rotundato-subcompresso in directionem leviter declivem, ad insertionem recto ; apertura subobliqua, lunata, declivi-subrotundato-ovata, intus candida; peristomate reclo, acuto, sat valide labiato, leviter subpatulescente, ad columellam mediocriter dilatato; alt. 9, diam. 12 millim.

Dunes auprès de Gabès (Berthier).

L'H. therella diffère de l'H. Tebourbana par son diamètre plus grand et sa forme par cela même moins conoide, la hauteur des deux espèces étant égale, par sa perforation profonde el ouverte, par" son dernier tour non anguleux à son origine, subarrondi à l'ouverture dans une direction déclive (celui de' l'éspèce précédente est exactement cylindrique), par son péristome marginalement bordé, non patulescent, etc.

14. Granmonensis Bourguignat, in Servain, Moll. Esp. p. 104, 1880 .

Celte Coquille, découverte sur les côtes océaniennes de France, retrouvée depuis en Espagne et en Algérie, a été recueillie, en Tunisie, sur le littoral prè̀s de Gabès (Let.).

H. thera Letourneux et Bourguignat, sp. nov.

Testa angustissime perforata (perforatio fere obtecta), subgloboso-conoidea, subpellucida, nitida, argutissime striatula, omnino candida, ad peristoma aurantiaca; spira producta, conico-convexa, ad summum obtusa (apex rufulus, minutus); anfractibus 6 convexiusculis, lente usque ad ultimum, deinde velociter crescentibus, sutura impressa, in ultimo impressiore, separatis; ultimo magno, ad aperturam- amplo, subcompressorotundato, súperne sat descendente; apertura obliqua, parurn lunata (margines approximati), declivi-semiovata, intus candida; peristomate aurantiaco, recto, valide crasseque labiato, patulo, ad columellan dilatato ac supra perforationem reflexo; alt. 11 , diam. 10 millim.

Golline aux environs de Gabès (Let.).

1. foedata Hagenmüller, sp. nov. 1881, et Locard, Prodr. Mal. Fr. p. 116 et 344,188 s.

Cetle Hélice, très répandue en Algérie (route de Bône à la Calle, Bougie, etc.), constatée également en France (plage d'Argelès-sur-Mer, dans les pyrénées-Orientales, fle de Sainte-Lucie, près de Narbonne, etc.), paraît abondante en Tunisie aux environs de Feriana et entre Haïdra et Tebessa dans les montagnes limitrophes de la frontière (Let.). 
H. agna Hagenmüller, sp. nov. 1881, et Locard, Prodr. Mal. Fr. p. 116 et 344,188.

Gette espèce, dont le type a été également découvert en Algérie (environs de Bône) et qui a été, de plus, constatée en France (île de Sainte-Lucie), ainsi qu'en Sicile (Trapani, Vizzini), semble commune aux environs de Tozer (Let.).

Cette Hélice offre dẹs variations de taille que nous devons mentionner; à, Béja (Hagenm.) et au Djebel Reças (Let.), on rencontre une variété major, tandis qu'à Tebourba (Let.) on trouve fréquemment une forme minor d'un tiers plus petite que le type.

Les douze Hélices que nous venons de signaler appartiennent au'groupe de l' $H$. maritima de Draparnaud (non des auteurs), dont les espéces sont répandues dans presque toutes les contrées du pourtour de la Méditerranée.

S 55.

I. Ferianica Letourneux et Bourguignat, sp. nov.

Testa perforata (perforatio parum profinda, in ultimo solum dilatata ac sat aperta), turrito-conica, opacula, striatula, omnino luteola; spira producta, conica, ad summum mamillata (apex ater, minutus, obtusus); anfractibus 6 convexis, regulariter usque ad ultimum, deinde rapide crescentibus, sutura impressa separatis; ultimo majore, cylindrìco, superne perlente descendente; apertura subobliqua, vix lunata (margines approximati), fere circulari; peristomate recto, acuto, non labiato, ad columellam leviter dilatato; alt. 14 , diam. 16 millim.

Environs de Feriana (Let.).

Aux alentours de Bône, l'on rencontre une variété plus petite, à péristome rougeảtre légèrement encrassé.

11. Sitifiensis Bourguignat, sp. nov. 1867 , et Locard, Prodr. Mal. Fr. p. 118 et 345 , 1882.

Bois de Pins au sud de Haïra et montagnes tunisiennes de la frontière entre Haidra et Lambessa (Let.).

Cette Hélice est très répandue en Algérie sur les hauts plateaux de Setif êt de presque toute la province de Constantine.

II. oreta Bourguignat, sp. nov. 1877 .

Testa perforata (perforatio in ultimo dilatato-aperta), globosa, opaca, nitida, striatula, in ultimo malleata, omnino candida, ad peristoma leviter rubella; spira convexo-rotundata, sat producta; apice rufo, minuto, , teviter prominente; anfractibus 6 convexiusculis, regulariter usque ad ultimum, deinde velociter crescentibus, sutura profunda separatis; ultimo maximo, ventroso, ad aperturam leviter declivi-rotundato, superne lente descendente; apertura vix obliqua, parum lunata (margines sat approximati), declivi-subrotundata, intus margaritacea; peristomate reclo, acuto, 
intus vix incrassatulo, ad columellam superne in triangulam formam dilatato; alt. 16 , diam. 23 millim.

Montagnes d'El-Kef à Souk-el-Arba (Let.).

Cette Hélice est abondante en Algérie (Setif, Aïnser-Aoumen entre Takitount-et Setif, plaine de Mélila près Batna, Djebel Sahari près Djelfa, Aïn-Oussera près Bou-Ghezoul, etc.). Elle existe également en Dalmatie aux environs de Zara.

\section{II. anephela Bourguignat, sp. nov.}

Testa late umbilicata (umbilicus in ultimo valde dilatatus), depressoglobosa cum spira subconica, solida, opaca, nitente, grosse striata ac passim malleata, uniformiter candida cum zonulis vix apparentibus; spira conico-depressa, ad summum acutiuscula (apex ater, minutissimus); anfractibus 7 convexiusculis, lente usque ad ultimum, deinde pervelociter crescentibus, sutura profunda separatis; ultimo maximo, amplo, globosorotundato, superne lente sat descendente; apertura obliqua, mediocriter lunata, semiovata in directionem subdeclivi-transversalem, intus candida; peristomate recto, acuto, intus valide labiato, leviter undique patulescente, ad columellam dilatato; alt. 16 , diam. 25 millim.

Environs de Tunis (Bourg. 1867).

Cette espèce est commune en Algérie dans la province de Constantine (Setif, Djebel Maadid entre la Medjana et le Hodna, sud de Biskra entre Setif et Cedraïat, etc.).

\section{II. pediana Bourguignal, sp. nov. 1883 .}

Testa profunde ac sat aperte umbilicata, globosa, solidula, opaca, nitente, grosse striata ac passim in ultimo malleata, candida; spira globoso-rotundata, relative valde producta, ad summum perobtusa (apex minutus, rufulus); anfractibus 7 convexis, regulariter crescentibus, sutura impressula separatis; ultimo rolundato, ab initio usque ad aperturam descendente; apertura parum obliqua ac lunata, subovato-rotundata in directionem leviter declivem, intus candido-margaritacea; peristomate recto, aculo, intus incrassatulo, in margine columellari validiore ac reflexo; alt. 23 , dian. 29 millim.

Environs de Bizerte (Berthier).

Chez cette espèce, le dernier tour, dont la direction descendante commence dès l'origine, finit par se trouver tellement en contre-bas, que l'avant-dernier forme ventre au-dessus de lui. Cette descente du dernier tour est un caraclère constant et non un signe accidentel particulier à quelques échantillons, puisque l'on rencontre très abondamment cette forme sur les hauts plateaux des environs de Setif et dans la plaine de Melila, au nord du chott Mzouri, toujours avec la même direction descendante.

Nous connaissons encore I' $H$. pediana aux alentours de Zara, en Dalmatie, et 
une variété minor (H. 15, D. 20 mill.) de cette Hélice sur les bords du Safsaf près de Philippeville, dans la province de Constantine.

I. pedianopsis Hagenmüller, sp. nov.

Testa profunde angusteque perforata, globosa, solida, opaca, nitente, eleganter striatula, candida; spira sát producta, perobtuso-rotundata, tholum simulante; apice millutissimo, rufulo; anfractibus 7 (superiores usque ad penultimum subtectiformes ac carinati [carina suturam sequens]; cæeteri [scilicet penultimus et ultimus] convexo-rolundati, celeriter crescentes, ac sutura profunda separati); ultimo magno, amplo, subovatorotundato ac superne e medio evolutionis usque ad aperturam regulariter et sat rapide descendente; apertura obliqua, parum lunata, oblonga in directionem declivem, intus albo-margaritacea; peristomate recto, intus crasso, labiato-subpatulescente, in columella valde dilatato; alt. i3, diam. 16 millim.

Collines près de Medjez-el-Bab (Hagenmüller).

Cette nouvelle forme se distingue de la précédente, non seulement par sa petite taille, son test plus épais, plus crétacé, plus régulièrement strié, son ouverture plus oblique, oblongue (non subovale-arrondie), entourée par un péristome épais labié-patulescent, mais encore par sa spire en forme de dôme, ses tours supérieurs carénés, enfin par son dernier tonr moins descendant que celui de l'H. pediana, puisque la direction descendante, chez cette espèce, ne commence qu'à moitié de sa circonvolution.

Ces six Hélices appartiennent au groupe de l'II. Melilana, dont les principales espèces, sans compter celles que nous venons de faire connaître, sont les $H{ }^{\circ} \mathrm{Cal}$ ventiana, Koleensis, 'Jusiana, Lucanti, Melilana, euetha ${ }^{\text {(1) }}$ (Bourg.) d'Algérie; nuporana, Toutana, Bourlieri, Tablatica (Let.) également d'Algérie.

\section{S 56.}

W. certa Bourguignat, sp. nov.

Testa profunde umbilicata (umbilicus pervius ac nihilominus in ultimo relative dilatatus), subglobosa ac leviter conoidali, candida, cretacea, opaca, nitida et striatula ; spira obtuse conica, mediocriter producta (apex ater, minutus); anfractibus 7 convexiusculis, lente usque ad ulimum, deinde rapide crescentibus, sutura impressa separatis; ultimo magno, dilatato, subcompresso-rotundato, ad insertionem leviter descendente; apertura obliqua, parum lunata, subsphærica in directionem leviter declivem, intus pallide ochracea; peristomate recto, acuto, intus incrassato ac profunde labiato, in columella superne dilatato; alt. 15 , diam. 22 millim.

(1) Cette espèce vient d'ètre décrite et signalée en Sieile par M. Je baron Corrado Cafici (Bull. Soc. malac. Fr. 1884). 
Cette espèce, découverte sur les hauts plateaux de Setif (province de Constantine), vit également aux environs de Haidra, où elle paraît rare.

H. caudefaeta Letourneux et Bourguignat, sp. nov.

Testa profunde umbilicata (umbilicus angustus, non pervius, sed modo in ultimo leviter dilatatus), globoso-conica, solida, opaca, nitida, sat valide striatula, candida, ad aperturam pallide subcarneola; spira obtuse conica, relative producta (apex rufus, exiguus); anfractibus 7 vix convexiusculis, lente usque ad ultimum, deinde rapidius crescentibus, sutura fere lineari, in ultimo impressa, separatis; ultimo majore, relative dilatato, subrotundato, ad insertionem bene descendente; apertura obliqua, parum lunata, subsphærica in directionem leviter declivem, intus subluteolo-candida ad marginem peristomalem carneola; peristomate recto, acuto, intus valide labiato, ad marginem columellarem crasso et reflexo; alt. $14 / / 2$, diam. 19 millim.

Environs de Haïdra (Let.).

Cette forme se distingue de la précédente par sa taille moindre en diamètre, ce qui la fait paraître plus haute et plus conique, par son ombilic moins ouvert, non en entonnoir, mais seulement faiblement dilaté au dernier tour, par ses tours supérieurs presque plans, comme tectiformes, et séparés par une suture pour ainsi dire superficielle, par son dernier tour moins dilaté, mieux arrondi et plus fortement descendant à l'insertion du bord supérieur, enfin par son péristome plus vigoureusement bordé, plus épais sur le bord columellaire, qui est réfléchi sur toute sa longueur, tandis que chez l'H. certa, il est dilaté seulement à la partie supérieure.

\section{I. leucophora Bourguignat, sp. nov.}

Testa profunde angusteque perforala, subcompresso-conoidali, solidula, leviter opacula, subtiliter striatula, nitida, candido-Jactescente aut lacteosubcœrulescente; spira parum producta, conoidali-tectiformi (apex"ater, minutus); anfractibus 7 vix convexiusculis, regulariter ac sat celeriter crescentibus, sutura vix impressula separatis; ultimo majore, ad initium leviter subangulato, ad aperturam rotundato, ad insertionem breviter descendente; apertura mediocriter obliqua ac lunata, hemisphærica, intus pallide ochracea; peristomate recto, acuto, intus labiato, ad columellam dilatato; alt. 11 , diam. 16 millim.

Environs de Tunis (Bourg. 1867); vallée de l'Oued Zergua (Hagenmüller); collines d'Hammam-el-Lif (Let.).

Aux alentours de Haïdra (Let.), on rencontre une variété un peu plus grande et à perforation quelquefois un tant soit peu plus ouverte.

Sa taille plus petite, son test moins calcaire légèrement transparent, sa surface finement striée, sa coloration, sa perforation étroite, sa spire tectiforme, son 
dernier tour très brièvement descendant à l'insertion, etc., sont autant de signes différentiels qui distingueront cette Hélice des précédentes.

\section{Ingenun Bourguignat, sp. nov.}

Testa relative exigua, pervie ac aperte umbilicata, subconico-globosa, solidula, opaca, nitida, striatula, candida; spira subtectiformi-conica, ad summum obtusa (apex minutus, ater); anfractibus 6 convexiusculis, regulariter crescentibus, sutura inpressula separatis; ultimo mediocriter majore, obscure subangulato-rotundáto, ad insertionem vix descendente; apertura parum obliqua et lunata, hemisphærica, intus candida; peristomate recto, acuto, intus valide labiato, ad columellam dilatato; alt. 9 , diam. 12 millim.

Sous les pierres entre la Mahmedia et les ruines d'Oudena (Bourg: 1967).

Cette Hélice diffère de l'II. leucophora, la seule de ce groupe qui puisse lui être assimilée, par sa taille noondre, par sa forme plus globuleuse, par son test plus épais d'une coloration différente, par sa croissance spirale plus régulière, par sou dernier tour à peine descendant, etc., et notamment par son ombilic largeinent ouvert en entonnoir.

\section{1. aceln Bourguignat, sp. nov.}

Testa aperte umbilicala (umbilicus in ultimo dilatatus), depressa, supra parum convexa, solida, opaca, nitida, striatula, candida; spira vix convexa, omnino depressa (apex ater, minutus); anfractibus 6 convexiusculis, lente usque ad ultimum, deinde rapide crescentibus; sutura profunda separatis; ultimo magno, dilatato, subcompresso-rotundato, ad insertionem brevissime leviter deflexo; apertura mediocriter obliqua ac lunata, transverse ovata, intus candida; peristomate recto, acuto, intus incrassato, ad columellam modo superne dilatato; alt. 10 , diam. 19 millim.

Cette Hélice, qui ne peut être confonduc avec aucune de ce groupe, à cause de sa forme déprimée, de sa spire presque plane, de son large ombilic, de son ouverture ovalaire dans le sens transversal, etc., vit aux environs de Tebessa (province de Constantine) et, en Tunisie, dans les alentours de Haidra, où elle est rare.

Ces cinq Hélices (H. certa, caudefecta, leucophora, ingenua, acela) auxquelles il faut joindre encore l'H. culaba (Bourg.) des environs deBou-Saada, forment ungroupe d'espèces intermédiaires entre celui de l'H. Melilana et la série des $H$. monerea.

\section{s 57.}

Les huit Hélices qui suivent font partie d'un groupe essentiellement tunisien, rèmarquable par ses espèces blanches, à test crétacé, sans caractères bien tranchés, mais, malgré tout, possédant un cachet particulier qui ne permet pas de les classer soit parmi les Hélices du groupe précédent, soit parmi celles de la série de l'H. subrostrala, dont aucun représentant n'a été constaté jusqu'à présent d'une façon cettaine dans la Tunisie. 
H. monerea Bourguignal, sp. nov.

Testa mediocriter umbilicata (umbilicus in centro profundus ac punctiformis, in ultimo leviter dilatatus), subglobosa, cretacea, opaca, striatula, nitida, uniformiter candida;-spira convexa, subdepresso-conoidali; ad summum leviter acutiuscula' (apex minutissimus, rufulus); anfractibus 6 t/2 convexiusoulis, regulariter usque ad ultimum crescentibus, sutura fere lineari, in ultimo impressula; separatis; ultimo magno, tumidorotundato, ad insertionem breviter'subdeflexo; apertura parum obliqua, mediocriter lunata, hemisphærica; peristomate recto, acuto, intus profunde valde labiato, inferne subpatulescente ac in columella validiore et mediocriter dilatato; alt. 15; diam. 21 millim.

Environs de Tunis dans les champs (Bourg. 1867), colline de Béja et vallée de roued Zergua (Hàgeum.); alentours de Ghardimaou (Pechaud).

Variété minor à Hammam-el-Lif (Berthier).

Cette espèce existe également en Algérie aux environs de Bou-Saada.

H. ehioldea Bourguignat, sp: nov.

Testa anguste profundéque umbilicata (úmbilicus in ultimo non dilatatus), globosa, sat conoidea, cretacea, opaca, nitida quasi polita, subtilissime striatula, uniformiter eburneo-candida; spira convexo-conoidea, sat elata, ad summum subacutiuscula (apex minutus, rufulus); anfractibus 6 convexiusculis, regulariter (in ultimo rapidius) crescentibus, sutura impressula separatis; ultimo majore, rotundato nihilominus ad initium subcompresso ac obscure subangulato, ad insertionem breviter deflexo aut aliquando subrecto; apertura subobliqua, lunata, hemisphærica in directionem leviter subdeclivem; peristomate recto, acuto, valide labiato ac leviter subpatulescente, ad columellam mediocriter relative dilatato; alt." 13 , diam. 17 millim.

Endroits aridés aux environs de Tunis (Bourg. 1867); Medjez-el-Bab dans la vallée de la Medjerda et vallon de l'Oued Meliana (Hag.); alentours de Haïdra (Let.).

Variété conoidea sur la colline de Béja (Hag.).

Cette forme se distingue de la précédente par sa taille moindre, par son test plus crétacé, plus brillant, plus poli, par son ombilic non évasé au dernier toưr, mais ressemblant à un trou profond et étroit, par son dernier tour moins développé, non aussi renflé-globuleux, mais légèrement comprimé et subanguleux à son origine, par son ouverture hémisphérique dans une direction légèrement déclive et par son bord péristomal plus robuste, dont l'encrassement intérieur est presque marginal.

4. phobela Letourneux et Bourguignat, sp. nov.

. In

Testa profunde sat anguste ac subpervie umbilicata, subglobosa, cre- 
tacea, opaca, nitidissima, subtiliter striatula, candida ac duabus zonulis atro-rufis (quarum una fere supera continua et altera inferior interrupta) eleganter cincta; spira convexa, ad summum obtusiuscula (apex minutus, rufulus); anfractibus 6 convexiusculis, regulariter crescentibus, sutura impressula separatis; ultimo mediocriter majore, exacte cylindrico, ad insertionem recto; apertura parum obliqua ac lunata, hemisphærica; peristomate recto, acuto, intus robuste incrassalo, inferne subpatulo, ad columellam mediocriter dilatato; alt. 10, diam. $13 \mathrm{millim}$.

Sur les pelouses arides du Djebel Reças (Let.). Espèce peu commune.

Cette Hélice diffère des deux précédentes par sa petite taille, par son test excessivement brillant sur lequel se détachent deux bandes très foncées, dont l'inférieure interrompue, par une spire convexe sensiblement obtuse au sommet, par une croissance spirale régulière, par un dernier tour bien cylindrique et rectiligne à l'insertion.

\section{H. Spllmenti Bourguignat, sp. nov.}

Testa profunde angusteque umbilicata, globosa-conoidea, subopacula, mediocriter cretacea, nitida, vix striatula, quasi polita, uniformiter candida; spira tectiformi-conoidali, ad summum acutiuscula (apex mamillatus, leviter prominens, rufulus); anfractibus 8 tectiformi-subconvexiusculis, lente usque ad ultimum, deinde mediocriter rapidius crescentibus, sutura sublineari separatis; ultimo majore, rolundato, ad aperturam declivi, ad insertionem subrecto; apertura obliqua, parum lunata, semiovata in directionem declivem; peristomate recto, acuto, intus incrassatulo, ad columell am modo superne subtriangulatim dilatato; alt. 9 , diam. 12 millim.

1. Cette petite espèce, découverte par. M. le sous-lieutenant Eugène Spilment sur la cỏte nord tunisienne entre le cap Roux et Tabarka, se distingue des H. monerea, chioidea et phabeia par son test moins crétacé, subopaque, presque lisse, par sa spire tectiforme-conoìde, surmontée d'un sommet mamelonné légèrement saillant, par son ouverture plus oblique semi-ovalaire dans une direction nettement déclive, par sá croissance spirale plus lente, par sa suture superficielle, etc.; enfin, par son dernier tour relativement moins développé que celui des trois Hélices précédentes.

\section{H. catarota Bourguignat, sp. nov.}

Testa profunde angusteque umbilicata, subventroso-depressa, crelacea, opaca, nitida, sat valide striatula, candida; spira parum convexa, perobtusa (apex exiguus, rufulus); anfractibus 6 convexiusculis, regulariter crescentibus, sutura sublineari separatis; ultimo majore, subrotundato, aliquando ad initium obscure subangulato, ad aperturam ampliore, ad insertionem breviter deflexo; apertura mediocriter obliqua ac lunata, subcirculari; peristomate recto, acuto, intus incrassato, inferne patulescente, 
ad marginem columellarem parum dilatato; marginibus conniventibus; alt. 11 , diam. 16 millim.

Cette Hélice, distincte des autres de ce groupe par sa forme déprimée, par sa spire peu convexe, très obtuse, par son test plus fortement strié, par sa croissance spirale régulière, par ses bords marginaux rapprochés et convergents, etc., se rencontre sur les pelouses arides des environs de Tunis (Bourg. ${ }^{186} 7$ ) et de Medjez-el-Bab (Hag.); ainsi que sur le versant du Djebel Bou-Kourneïn près Hammam-el-Lif (Let.).

I. catarotella Bourguignat, sp. nov.

Testa profunde ac sat aperte umbilicata, depressa, æque convexa supra ac infra, cretacea, opaca, nitida, polita, subtilissime striatula, omnino candida; spira depressa, convexo-rotundata, perobtusa (apex minutus, rufulus, leviter prominens); anfractibus 6 convexiusculis, regulariter crescentibus, sutura sublineari separatis; ultimo majore, subrotundato-compresso, superne lente descendente; apertura subobliqua et lunata, subrotundata; peristomate recto, acuto, intus robuste incrassato-labiato, inferne patulescente, ad columellam mediocriter dilatato; alt. 9, diam. 14 millim.

Ruines d'Oudena et environs de Tunis (Bourg. 1867).

Variété major, sur la colline de Béja (Hag.).

Gette espèce se distingue de l'H. catarota par sa taille moindre, par sa forme un peu plus déprimée, par sa spire moins convexe, à sommet légèrement saillant, par son ombilic plus ouvert, par son dernier tour lentement descendant et non pourvu d'une déflexion courte comme celle de l'H. catarota, par son ouverture un peu moins oblique et moins exactement circulaire.

H. cana Bourguignat, sp. nov.

Testa anguste umbilicata (umbilicus in centro punctiformis, in ultimo dilatatus), depressa, supra convexa, infra convexiore, cretacea, opaca, nitida, polita, subtiliter substriatula, candida; spira parum convexa, perobtusa (apex minutus, ater); anfractibus 6 vix subconvexiusculis, lente usque ad ultimum, deinde rapide crescentibus, sutura sublineari, in ultimo impressula, separatis; ultimo magno, rotundato, ad initium subcompresso ac obscure subangulato, ad aperturam ventroso-rotundato, ad insertionem leviter descendente; apertura mediocriter obliqua ac lunata, ampla, semicirculari, æque alta ac lata; peristomate recto, acuto, intus valide incrassato, externe inferneque (superne excepto) patulescente ac ad columellam dilatato; alt. 10 , diam. 16 millim.

Ruines d'Oudena (Bourg. 1867); environs de Haïdra (Let.).

Cette Helice diffère de l'H. catarotella par sa taille plns forte, par sa forme plus déprimée, par son ombilic plus étroit, par son dernier tour plus dilaté, plus ren- 
flé ver's l'ouverture et sensiblement plus haut; enfin, notamment, par son ouverture ample, aussi liaute que large, bien circulaire, à bord supérieur exactement convexe et non incliné comme celui de l'espèce précédente.

H. Ieucestha Bourguignat,'sp. nov.

Tesla profunde angusteque umbilicata, depressa, nihilominus ventrosa, cretacea, opaca, nitida, polita, subtiliter striatula, uniformiter candida; spira depressa, parum convexa (apex minutus, rufulus); anfractibus 6 subconvexiusculis, regulariter ac sat celeriter (præsertim in ultimo) crescentibus, sutura fere lineari separatis; ultimo magno, alto, ad initium subcompresso obscureque subangulato, deinde rotundato ac ad aperturam amplo et ventroso, inferne circa umbilicum subturgido, superne ad insertionem recto; apertura vix obliqua ac lunata, ampla, semicirculari, æque alta ac lata; peristomate recto, aculo, labiato, leviter patulescente ac ad columellau robustam dilatato; alt. 9 , diam. 15 millim.

Cette espèce, quil se distingue de l'H. cana par sa spire encore plus déprimée, par son ombilic plus étroit par suite de la turgescence circumombilicale du dernier tour, par sa croissance spirale plus régulière, par son dernier tour rectiligne supérieuremeıt, plıs ample et plus ventru sur l'ouverture, qui est plus grande el un peu moins oblique, etc., a été également recueillie, en 1867 , dans les ruines d'Oudena au sud de Tunis (Bourg.).

\section{8.}

Ce groupe des H. Latastei est, pour la Tunisie et le sud des provinces de Constantine et d'Alger, ce qu'est celui des II. subrostrata pour la province d'Oran et la partic occidentale de celle d'AJger. Les Hélices de ce groupe abondent dans le sud de la Régence, ainsi que dans la région de Djelfa (sud de la province d'Alger). Elles paraissent rares entre ces deux stations; néanmoins, fait singulier, une espèce Latastéenue, I'L. Mahdarina, existe près d'Oran.

Les fornies de ce groupe sont caractérisées par un test globuleux, bien strié, souvent rugueux, surmonté d'un sonmet saillant, formant pointe. Cinq espèces nous sont connues, deux de l'Algérie, les $I$. Malidarina ${ }^{(1)}$ et $n y a^{(2)}$ et trois de la Tunisie.

(1) Helx Mambirima (Bourg.). Testa aperte profunde ac leviter pervie pcrforata, subglobosodepressa, solidula, opacula, nitida, arcte striata, candida cum zonulis 4 rufo-continuis, quarum medianæ 2 , infcriores a angustiores; spira convexa, ad summum acuta (apcx rufo-opalinus, valde prominens); anfractibus 6 convexis, sat celcriter, præsertim ad ultimum, crescentibus, sutura impressa separatis; ułtimo magno, rotundato-globoso, superne longo lentcque descendente ac ad insertionem rapidius deflexo; apertura sat obliqua, lunata, subrotundata, inferne leviter subrectiuscula, intus margaritacea cum zonulis apparentibus; peristomate recto, acuto, intris valde labiato, inferne patulo, ad columeltam robustam medjocriter dilatato; alt. 11, diam, 16 millim. - Bords de l'Oued Mahdar près Oran.

(2) Heux vy (Let.). Testa punctiformi-perforata, parvula, globosa, solida, opacula, nitida, subtiliter striata, candida cum zonnlis 3 ant 4 vix apparentibns; spira parum etata, nihilominus 
H. Latastei Letourneux, sp. nov.'

Testa anguste perforata (perforatio sæe fere subnulla), globosa, opacula, sæpe sat fragili, nitidiuscula, valide striata quasi rugosa, albida aut subalbido-obscure-violacea ac zonulis rubro-rufis (quarum superiores duæ, una circa suturam interrupta, altera continua; inferiores 1-4 interruptæ quarum 3 plerumque evanidæ) cincta; spira convexa, plus minusve subconoidea, ad summum acuta (apex ater, semper prominens); anfractibus 6 convexis, regulariter crescentibus, sutura impressa separatis; ultimo majore, globoso-rotundato, ad insertionem leviter descendente; apertura vix obliqua, lunata, hemisphærica, intus lulescente aut vinosa cum zonulis apparentibus; peristomate recto, acuto, intus mediocriter labiato, leviter patulescente, ad columellam subdilatato; àlt. 11-13, diam. 1416 millim.

Cette belle Hélice, dédiée à M. Lataste, membre de la mission, est excessivement répandue dans tout le sud de la Tunisie, où elle se trouve sur les plantes et les arbustes.

Ketenna et dans le vallon de l'Oued El-Ftour, ainsi qu'à l'oasis du Hammam de Gabès. Plaine entre Ras-el-Aïn et Sidi-Salem-Bouguerara. Bir-el-Ahmar. Bords de l'Oued Medzesar et de l'Oued Taferma entre Ain-Magroun et Fratis. Ras-ed-Djerf, vis-à-vis de Djerba; Zarzis, etc. (Let.).

Aux environs de Gabès, à Ketenna et dans Ja plaine entre Ras-el-Ain et SidiSalem-Bouguerara, on rencontre deux variétés, l'une à spire plus conique, l'autre à spire déprimée.

En Algérie, l'H. Latastei a été constatée aux Ouled Naïl près de Biskra où elle est fort rare, à Aïn-Oussera, à Bou-Ghezoul sur les hauts plateaux, entre Boghar et Laghouat et entre cette ville et Djelfa, où elle est abondante. Les échantillons de ces dernières localités sont ordinairement sans bandes.

\section{W. Latasteopsis Letourneux et Bourguignat, sp. nov.}

Testa anguste perforata, tumido-globosa, subopacula, nitidiuscula, valide striata, in ultimo qausi corrugata, albidula ac zonulis fere evanidis circumcincta; spira convexo-rotundata, producta; ad summum acuta (apex minutus, rufus, valde prominens); anfractibus 6 convexis, regulariter usque ad ullimum, deinde velociter crescentibus, sutura sat profunda separatis; penultimo pertumido-ventroso; ultimo relative mediocri, nihilo-

subconica ${ }_{2}$ ad summum acutiuscula (apex exiguus, rufulus, mediocriter prominens); anfractibus 5 convexiusculis, lente usque ad ultimum, deinde rapide crescentibus, sutura impressa separatis; ultimo maximo, ad initium subcompresso, ad aperturam amplo, turgido-rotundato, ad insertionem leviter subdescendente; apertnra subobliqua, ampla, circulari, leviter magis alta quam lata, intus margaritacea; peristomate recto, acuto, pervalide labiato ac undique patulescente, ad columellam superne triangulatim dilatato; alt. 8, diam. 10 1/9 millim. - Colliues entre Djelfa et Mesran (sud de la province d'Algrr). 
minus majore, rotundato, regulariter valdeque e dimidie evolutionis descendente; apertura subobliqua ac lunata, subcirculari, intus lutescente; peristomate recto, acuto, subincrassatulo, ad columellam mediocriter dilatato; alt. 16 , diam. 20 millim.

Gette Hélice, remarquable par la forte descente de son dernier tour; descente qui commence à s'accentuer à partir de la moitié de la circonvolution du tour, a été trouvée à Foum-Hallouf et à Ras-ed-Djerf, vis-à-vis de Djerba (Let.).

H. aestuosa Berthier, sp. nov.

Testa anguste profundeque perforala, parvula, globoso-conica, opacula, nitida, sat subtiliter striatula, circa suturam rugosiuscula, albidula cum zonulis numerosis, angustis, parum perspicuis; spira conica, sat producta, ad sunmum acutiuscula (apex minutus, rufulus, mediocriter prominens); anfractibus 6 convexiusculis, regulariter crescentibus, sutura impressula separatis; ultimo majore, rotundato, superne vix leviter lenteque descendente; apertura obliqua, lunata, semicirculari, intus margaritacea; peristomate recto, acuto, intus rubello-labiato, ad columellam vix dilatato; alt. 8 , diam. 10 millim.

"Cette forme, qui se distingue des deux précédentes par son test plus finement striolé, par son sommet moins saillant, par son mode de coloration, par sa petite taille, par sa suture plus superficielle, ainsi que par ses tours moins convexes, etc., a été recueillie sur la plage près de Gabès (Berthier).

\section{S 59.}

La série de l'H. acompsia, qui comprend, à notre connaissance, sept espèces, parmi lesquelles nous citerons les H. aspila (Bourg.) et acompsella (Ancey) d'Algérie, l'I. cornarina (Berthier) de l'ille de Rhodes, l'H. Heracleana (Bourg.) de l'ile de Crète, est représentée en Tunisie par les trois formes suivantes.

1. acompsia Bourguignat, Mal. Alg. I, 1864 , p. ${ }_{21} 7$, pl. XXIV, fig. ${ }^{1-1} 9$ (excl. fig. 20-31).

Environs de Feriana (Let.).

En Álgérie, cette belle espèce a été constatée aux alentours de Bône, à l'embouchure du Safsaf près Philippeville (localité type), aux alentours d'Oran, à Saint-Denis-du-Sig et dans lá vallée du Rio-Salado, enfin dans les clamps près de Tlemcen.

H. etsema Letourneux et Bourguignat, sp. nov.

Testa punctiformi-perforata (perforatio semitecta), globoso-conoidea, opacula, nitida, candida, subtiliter striata (striæ in ultimo sæpe malleato validiores) ac lineis spiralibus minutissimis pernumerosisque circumcincta; spira producta, conoidea, ad summum obtusiuscula (apex rufulus, splendeus, mediocris); anfractibus 6 convexiusculis (superiores carinati 
[carina suturam sequens]), regulariter erescentibus, sutura impressula separatis; ultimo magno, ventroso, rotundato, nihilominus ad inilium obscure subangulato, ad insertionem recto; apertura parum obliqua, lunata, semirotundata; peristomate recto, acuto, intus labiato, ad columellam præsertim superne dilatato; marginibus renotis; att. 14, diam. 15 millim.

Région des Hamada (Let.). Espèce rare.

Cette Hélice se distingue de l'H. acompsia par sa forme plus conique, par sa perforation ponctiforme, par ses tours moins convexes, par sa suture moins prononcée, par son ouverture plus échancrée, par ses bords plus distants, par sa dilatation columellaire plus accusée, par son mode différent de striation, etc.

\section{H. amoma Bourguignat, sp. nov.}

Testa aperte pervieque umbilicata, subgloboso-conoidea, solidá, opacula, non nitente, candida, arcte valideque striata ac leviter quasi rugosa; spira convexo-subconoidea, sat elata, ad summum obtusiuscula (apex ater, minutus); anfractibus 6 convexiusculis, regulariter crescentibus, sutura impressa separatis; ultimo majore, exacte cylindrico, ad insertionem breviter parum descendente; apertura subobliqua ac mediocriter lunata, subcirculari, intus splendide margaritacea; peristomate recto, acuto, intus uni- aut bilabiato,"inferne patulo, ad columellam mediocriter dilatato; alt. 12 , diam. 17 millim.

Ruines d'Oudena près Tunis (Bourg. 1867).

En Algérie, cette espèce a été recueillie sur les dunes de la Macta (localité type), à Saint-Denis-du-Sig près Oran, dans la forêt d'Afir au sud de Tlemcent et à Oumel-Adham à quarante kilomètres au sud de Ksar-el-Bokhari.

Son large ombilic en entonnoir (sans compter ses autres signes différentiels que nous croyons inutile d'énumérer) suffit pour distinguer cette Hélice des deux précédentes.

\section{$\$ 60$.}

Les formes de ce groupe, au nombre de huit, sont toutes spéciales aux régions nord-occidentales de l'Afrique. Sur ces huit formes, toutes ont été retrouvées en Tunisie, sauf l'H. akranica (Pechaud) de la province d'Oran et l'H. chloramaura (Let.) de Teniet-el-Háad' (province d'Alger).'

Ces Hélices giobuleuses, à spire plus on moins subconoïde, sont remarquables par leur test calcaire, épais, d'un blanc sale, par leurs tours tectiformes (séparés par une suture superficielle), à l'exception du dernier relativement très ventru, et par leur ouverture semi-ronde entourée d'un bord péristomal fortement encrassé et patulescent.

\section{H. panurga Bourguignat, sp. nov.}

Testa plus minusve anguste perforata, globoso-conoidea, solida, opaca, Malacologie. 
cretacea, parum nitente, uniformiter candida (aliquando, in speciminibus algeriensibus ${ }^{(1)}$, in ordines interruptos obscure castaneo-maculata), valide striata quasi costulata (striæ regulares, plus minusve distantes, aliquando in ultimis leviter crispulatæ); spira obtuse conoidea, producta, ad summum rolundata (apex minutus, ater, splendens); anfractibus 7 tectiformi-declivibus, carinatis (carina non perspicua, suturam sequens), regulariter usque ad ultimum, deinde rapidius, præsertim ad aperturam, crescentibus, sutura lineari, in ultimo leviter impressula, separatis; ultimo magno, tumido, plus minusve angulato (in speciminibus tingitanis subrotundato), superne subconvexo-tectiformi, inferne convexo, ad aperturam ampliore, ad insertionem labri breviter deflexo; apertura parum obliqua; lunata, semirotundata; peristomate recto, intus robuste incrassato, fere undique patulescente, ad columellam superne dilatato; alt. 18 , diarm. 22 millim.

Environs de Tunis (Bourg. 1867).

Cette Hélice a encore été constatée à Saint-Denis-du-Sig et à Nemours, dans la province d'Oran, ainsi qu'aux environs de Tanger (Pechaud).

Cette forme se distingue de la suivante, l'I. euphorca, par son test plus épais, plus calcaire, par sa forme plus conoide, par son dernier tour subanguleux, surtout par sa spire élevée et ses tours tectiformes du sommet à l'ouverture, caractère qui imprime au bord supéro-apertural un aspect subrectiligne et donne aux contours de l'ouverture une forme différente.

H. cuphorea Bourguignat, Malac. Alg. I, 1864, p. 233 , pl. XXV, fig. 2 i, 24.

Cette espèce est très exactement représentée à la planche XXV de la Malacologie de rAlgérie. Elle a été primitivement trouvée dans des détritus de graines abandounés sur le port d'Alger. Depuis elle a été constatée dans la province d'Oran à Perrégaux, Tlemcen et Lalla-Maghnia, et, dans la province d'Alger, aux Attaf entre Affreville et Orléansville:

En Tunisie, elle a été trouvée (Bourg. 1867) dans les campagnes arides des alentours de Tunis, où, quelquefois, elle se présente sous une coloration uniforme plus ou moins roussâtre ou sous une apparence zonulée.

\section{n. euphoreella Pechaud, sp. nov. 1882.}

Testa anguste perforata, tumido-globosa, supra in tholum producto-rotundata, cretacea, leviter opacula, non nitente, subtiliter valideque striata, sordide lutescente cum zonulis rufis angustis plus minusve interruptis ac saturatis (rare candida cum zonulis); spira in tholum producta, convexorotundala, ad summum obtusa (apex minutus, ater, leviter prominens); anfractibus 6 tectiformi-subconvexis, carinatis (carina suturam sequens),

(1) De Nemours et de Saint-Denis-du-Sig (province d'Oran). 
regulariter usque ad ultimum, deinde rapidius crescentibus, sutura lineari in ultimo subimpressula separatis; ultimo magno, inflato, exacte cylindrico, superne breviter descendente; apertura parum obliqua, lunata, hemisphærica, intus subgriseo-lutescente aut subochracea cum zonulis violaceis apparentibus; peristomate recto, intus robuste incrassato ac patulescente, ad columellam dilatato; alt. 17 , diam. 20 millim.

Collines entre Mahmedia et Hammarn-el-Lif, et région des Hamada (Let.).

En Algérie, cette Hélice a été constatée notamment dans la province d'Oran aux environs de Tenez, Perrégaux ${ }^{(1)}$, Saint-Denis-du-Sig, Lalla-Maghnia et Tenira au sud de Sidi-bel-Abbès (Let. et Pechaud).

Cette espèce est plus globuleuse et plus renflée dans toutes ses parties que j' $H$. euphorca, dont elle diffère, en outre, par son dernier tour plus gros, exactement cylindrique, par son ouverture plus ample, plus régulièrement sphérique, par son test moins épais, par sa coloration, etc.

\section{H. esnorca Letourneux, sp. nov. 1876 .}

Testa aperte perforata (perforatio in ultimo sat dilatata), subconicoventrosa, nihilominus leviter depressa, cretacea, opaca, sat nitida, striata, in ultimo malleata, omnino candida (in unico specimine obscure subzonulata); spira perobtuse conoidea (apex minutus, rufulus); anfractibus 7 tectiformi-subconvexis usque ad ultimum, carinatis (carina suturam sequens), primo lente deinde rapide crescentibus, sutura lineari, in ultimo sat impressa, separatis; ultimo amplo, rotundato, superne perlente longeque descendente; apertura obliqua, lunata, semirotundata in directionem leviter declivem; peristomate recto, intus profunde incrassato, ad basim modo leviter patulescente, ad columellam dilatato; alt. 16, diam. 21 millim.

Le type de cette Hélice se trouve à Tebessa (province de Constantine). Seule, une variété minor a été rencontrée en Tunisie aux environs de Haïdra (Let.).

Cette espèce se distingue nettement des précédentes par sa perforation ouverte, dilatée au dernier tour, par son ouverture plus oblique et semi-ronde dans un șens incliné assez prononcé, par son bord péristomal enfoncé et seulement patulescent à la base aperturale, par son dernier tour moins renflé-globuleux ayant une apparence légèrement comprimée, bien que régulièrement arrondi et offrant supérieurement une longue direction descendante.

\section{II. meticulosa Letourveux et Bourguignat, sp. nov.}

Testa aperte perforata (perforatio in ultimo dilatata), conoideo-subdepressa, solida, opaca, cretacea, striata (striæ in ultimo validiores), omnino

(1) Les échantillons de cette localité nous sont souvent parvenus sous l'appellation erronée d'Hclix Cretica, imposée par M. Kobelt. 
candida; spira subfusiformi-conoidea, mediocriter producta (apex minutus, iufulus, nitidus); anfractibus 6 vix convexiusculis, regulariter crescenlibus, sutura impressula separatis; ultimo majore, ad initium subangulato ac sat depresso, ad aperturam rolundato, ad insertionem recto; apertura obliqua, lunala, lıemisphærica; perislomate reclo, inlus robuste incrassato, palulescente, ad columellam bene dilatato; alt. 13, diam. 18 millim.

Environs de Haïdra (Let.).

¿.. Sa taille moindre, sa forme plus déprimée, tout en restant aussi conoïle, son test plus épais, plus crétacé, sa suture non linéaire, sa croissance spirale régulière, sòi dernier tou compirimé-subanguleux à son origine et offrant en dessus unè direction parfaitement rectiligne, etc., sont antant de signes particuliers qui distingueront cette Hélice de la précédente.

II. euphorcopsis Letouneux in Ancey, Esp. nouv. syst. curop. in Bull, Soc. Malac. Fr. p. $158,1884$.

1)'Et-Kef is Souk-el-Arba (Let.).

En Algérie, cette jolic forme, distinguée avec raison par M. C.-F. Ancey, a été constatre en Kalyglie, au Fort-Napoléon, et dans la province d'Oran, à Nemours et à Henniäa iu nord-ouest de Tlemcen.

\section{$\$ 61$.}

Les Hélices de ce groupe sont des coquilles globuleuses, crétacées, opaques, spéciales à l'Algérie et à la Tunisie (1), ressemblant comme aspect aux espècés de la séric de l'II. Sruticuim. Elles sont dans l'ensemble des Hélices xérophiliennies, ê que sont les $I$. fruticum dans celui des H. fruticola de Held.

Sur les neuf espèces conmues, les II. Boughezoulensis, mutclla et Savariun (Let.), crissana et nipora (Bourg.), Lepinci (Berthier) sont algériennes; deux autres (II. Hadrumetomun et Urbarana) sont communes à I'Algéric et ì la Tunisie; une seule (l'll. euçana) paraît jusqu'à présent spéciale à la Régence.

11. Iadrunetorum Letourneux et Bourguignat, sp. nov.

Tesla anguste perforala, lumido-globosa, crelacea, opaca, solida, striata (stria plus minusve validiores), candida aut sordide albido-sublutescente (in specimine unico algeriensi zonuli angusta atraque circuncincla); spira producta, rolundato-convexa, oblusa (apex rufulus, minutus); anfractibus 6 conıexis, sal regulariter crescentibus, sutura impressula separalis; ultimo tumido-cylindrico, perlente e dimidio cvolutionis descendente; apertura obliqua, lunata, semirolundata; peristomate recto, aculo, intus robuste labialo-incrassalo, palulesceute, ad columellam mediocriler dilatato; alt. 14-15, diam. 17-19 millim.

II) L'une d'elles (l'Uibaruna) a èté mème constatie dans l'ìle de Crète, 
De Sousa à Monastir et à Mahdia (Let.).

En Algérie, cette Hélice existe aux Attaf (province d'Alger) entre Affreville et Orléansville, et à Saint-Lieu près d'Arzew (province d'Oran).

I. Urbarana Pechaud, sp. nov. 1882 .

Testa aperle perforala (perforatio in ultimo dilalata), globulosa, cretacea, opaca, solida, nitida, immaculata, candida ac striatula (striæ in ultimo crispulalæ); spira producta, subconoidali-convexa, ad summum minus obtusa (apex minutus, rufulus); anfractibus 6 convexiusculis, regulariter usque ad ultimum, deinde celeriler crescentibus, sulura impressula separatis; ullimo magno, subrotundato, leviler vix subcompressiusculo, superne ad insertionem sat valide descendente; apertura parum obliqua ac lunata, semiovata in directionem transversalem; margine supero leviter declivi; margine infero subrectiusculo; peristomate reclo, acuto, intus perrobuste incrassalo, patulescente, ad columellam parum dilatato; alt. 16 , diam. 21 millim.

Le type se trouve à Nemours (Pechaud) dans la province d'Oran. En Tinnisie, cette jolie espèce a été recueillie sur les collines près de Tebourba (Berthier).

L'H. Urbarana se distingue de l'H. Iladrumetorum par sa taille plus forte, par son test brillant d'une blancleur éclatante et immaculée, par ses striations plus délicates, par sa perforation dilatée au dernier tour, par sa croissance spirale moins régulière, par ses tours moins renflés, moins exactement globuleux-cylindriques, notamment le dernier, qui paraít un tant soit peu comprimé, par la direction descendante de son dernier tour beaucoup moins longue, par son ouverture non hémisphérique mais subovale dans une direction transversale, elc.

\section{II. eucana Hagenmüller, sp. nov.}

Testa profunde angusteque perforata, globoso-tumida quanivis spira depressa sit, cretacea, opacula, nitida, subtilissime striatula quasi polita, in ultimo malleata, uniformiter candida; spira depressa, parum elata, nihilominus conoidali-convexa (apex minutus, rufulus); anfractibus 7 convexiusculis, lente usque ad ultimum, deinde rapide crescentibus, sutura impressula separatis; ultimo maximo, amplo, inflato-rolundalo, superne ad, insertionem descendente; apertura obliqua, lunata, relative ampla, hemisphærica, æque alta ac lata; peristomate recto, acuto, intus profunde mediocriter incrassato, vix patulescente, ad columellam valde dilatato; alt: 14 , diam. 20 millim.

Colline de Béja (Hagenmüller).

Variété minor (haut. 11, dianı 15 mill.) dans la vallée de l'Oued Zergta (Hag.).

Sa spire déprimée, bien que conoïde, le développement de son dernier tour et son fort renflement. non moins que l'amplitude de son ouverture exactement hémisphérique, aussi haute que large, sont autant de signes caractéristiques qui distingueront cette espèce des deux précédentes." 
\$ 62.

Les sept Hélices de ce groupe, H. nemorta (Berthier), euleuca et Nemoursensis (Pechaud), microspila, Usarina et megistostoma (Bourg.), Maxulana (Let. et Bourg.), sont des coquilles également crétacées, opaques, remarquables par la dépression de leur spire, par le développement, relativement énorme, de leur dernier tour, et notamment par l'amplitude de leur ouverture, dont ta hauteur dépasse presque toujours la largeur. Le type de ce groupe, l'H. nemorta, habite en Grète et en Algérie, où il a été trouvé à Hennaīa près de Tlemcen. Quant aux autres, elles n'ont été constatées jusqu'à présent qu'en Algérie et en Tunisie.

\section{H. mierosplla Bourguignat, sp. nov.}

Testa anguste perforata, supra depressa, nihilominus in ultimo globosa, cretacea, solida, opaca, nitida, striatula, quasi polita, uniformiter candida (in specimine unico algeriensi lutescente cum zonulis rufulis numerosis ac superne circa suturam flammulis variegata); spira depressa, tectiformi, parum convexa (apex rufulus, minutissimus); anfractibus 6 tectiformi-declivibus, vix convexiusculis, carinatis (carina inconspicua suturam sequens), lente usque ad ultimum, deinde rapide crescentibus, sutura lineari separatis; ultimo maximo, ad initium angulato, ad aperturam inflato-rotundato, ad insertionem breviter subdeflexo; apertura obliqua, lunata, hemisphærica, leviter magis alta quam lata; peristomate recto, acuto, intus robuste incrassato, patulescente, ad columellam dilatato; alt. 12 , diam. 17 millim.

Lieux arides aux environs de Tunis (Bourg. 1867).

En Algérie, cette forme a été rencontrée au sud de Boghar sur les platẹaux entre Ain-Ouessera et Bou-Ghezoul (Let.) et près des Attaf entre Affreville et Orléausville (Pechaud).

H. Maxulana Letourneux et Bourguignat, sp. nov.

Testa profunde umbilicata (umbilicus subpervius), convexo-globosa, subcretacea, opacula, nitida, sat valide striata (striæ circa suturam validiores, infra tenuiores), uniformiter candida; spira depressa, in tholum rotundata (apex minutus, rufulus); anfractibus 6 convexiusculis, fere regulariter crescentibus, sutura sublineari, in ultimo impressula, separatis; ultimo majore, cylindrico, ad insertionem recto; apertura obliqua, lunata, hemisphærica, leviter magis alta quam lata, intus lutescente; peristomate recto, acuto, intus incrassatulo, ad columellam sat tenuem dilatato; alt. 11 , diam. 17 millim.

Collines près d'Hammam-el-Lif (Let.)

Cetle Hélice est très distincte de la précédente, grâce à son ombilic biẹn ouvert, à sa spire arrondie en dessus en forne de dòne, à son test moins épais, plus for- 
tement strié, à sa croissance spirale plus régulière, à son dernier tour moins gros , non anguleux à l'origine, à ses bords péristomaux faiblement bordés et non patulescents.

\section{$\$ 63$.}

Chez les espèces de ce groupe, qui toutes sont d'assez grandes Hélices ayant une certaine ressemblance de forme avec l'H. astata (mentionnée page 40), le bord péristomal est épaissi par un bourrelet blanc toujours profondément situé dans l'intérieur. Entre ce bourrelet et la tranche marginale, la paroi du péristome se développe mince, tranchante et teintée d'une coloration vineuse-rosacée ou marronrougeâtre. Le test blanchâtre (rarement zonulé), assez résistant, est peu épais et subopaque; la spire est élevée-conoïde ${ }^{(1)}$; le dernier tour est très développé; enfin l'ombilic, toujours médiocre, ne peut être considéré que comme une perforation.

A l'exception d'une forme italienne, l'H. Basilicatana.(Berthier, 1880), toules les autres paraissent spéciales aux hautes vallées du bassin de la Medjerda.

\section{Mayeti Letourneux et Bourguignal, sp. nov.}

Testa anguste perforata (perforatio parum profunda, in ultimo modo leviter aperta), globoso-convexa, solidula, opacula, sat nitente, subtiliter striatula (quasi polita), in ultimo sæpius plus minusve malleata, unilormiter albidula, ad peristoma obscure rufo-grisea, rare zonulis subrufulis interruptis circumcincta; spira convexa, obtuse conoidea (apex minutus, rufulus); anfractibus 7 parum convexiusculis, carinatis (carina vix perspicua, suturam sequens), regulariter usque ad ultimum, deinde velociter crescentibus, sutura sublineari, in ultimo impressula, separatis; ultimo maximo, tumido-rotundato, superne lente subdescendente; apertura parum obliqua, mediocriter lunata, hemisphærica, æque alta ac lata; peristomate recto, acuto, sat tenui, intus rosaceo-vinoso ac profunde albolabiato, ad columellam leviter dilatato; alt. 17, diam. 23 millim.

Cette espèce, dédiée à M. Valery-Mayet, professeúr à l'École d'agriculture de Montpellier et membre de la mission scientifique, a été primitivement découverte aux environs de Souk-Harras et de Tebessa, dans la province de Constantine (Let.), puis a été recueillie dernièrement, en Tunisie; aux alentours de Ghardimaou (Pechaud).

Chez l'H. Mayeti, le bourrelet péristomat est ordinairement enfoncé de 5 à 6 millim. dans l'intérieur.

11. Valeryana Letourneux et Bourguignat, sp. nov.

Testa anguste perforata (perforatio profundior), minus globosa, solidula, opacula, nitente, subtiliter striatula (striæ ad aperturam validiores), albidula et aliquando zonulis rufulis plus minusve evanidis ac numerosis

(I) Sauf chez les $H$. pachestha et Charmesiana oú elle est arrondie. 
cincta; spira convexa, obtuse conoidea (apex ater, minutus); anfractibus 7 convexiusculis, carinatis (carina non perspicua, suturam sequens), l'egulariter usque ad ultimum, deinde pervelociter crescentibus, sutura lineari, in ultimo impressula, separatis; ultimo maximo, non tumido, sed ovato-rotundato, ad aperturam ampliore, superne longe perlenteque descendente; apertura parum obliqua ac lunata, ovata in directionem leviter declivem, intus pallide rufulo-vinosa; peristomate recto, intus castaneo, profunde bilabiato, inferne leviter subpatulescente, ad columellam bene dilatato; alt. 18 , diam. 25 millim.

Environs de Ghardimaou (Pechaud), de Tunis (Berthier).

Variété minor à Fernana (Let.).

Cette Hélice, dédiée au même membre de la mission, M. Valery-Mayet, se distingue de la prédéde par son dernier tour moins renflé en liauteur, plus dilaté dans le sens transversal et présentant supérieurement une direction descéndante beaucoup plus longue, par son ouverture non hémisphériquẹ ni aussi haute que large, mais au contraire ovalaire dans un sens légèrement incliné et plus large que haute, par'ses deux bourrelets dont le plus externe est peu éloigné de la tranche marginale, par sa perforation plus profonde, par son bord columellaire plus robuste et plus dilaté.

\section{II. eumona Letourneux et Bourguignat, sp. nov.}

Testa sat profunde perforata, conoideo-globosa, solidula, opacula, nitente, striatula, uniformiter albidula aut rarius albido-zonata (zonæ rufæ 6-7 continuæ); spira sat elata, subtectiformi-conoidea (apex ater, minutus); anfractibus 7 vix subconvexiusculis, carinatis (carina inconspicua, suturam sequens), regulariter usque ad ultimum, deinde rapide crescentibus, sutura lineari ad aperturam impressula separatis; ultimo maximo, rotundato, ad aperturam ampliore, supra non convexo sed tectiformi-declivi, ad insertionem valide descendente; apertura obliqua, parum lunata, oblonga in directionem decliven; margiuibus (supero et columellari) subrectis ac subparallelis; peristomate recto, acuto, intus vinoso ac sat profunde albo-labiato, ad columellam superne valde dilatato; marginibus sat approximatis; alt. 17 , diam. 23 millin.

Collines près Ghardimaou (Pechaud).

Environs de Tebessa (Let.) dans la province de Constantine.

Cette forme est très remarquable par l'amplitude de son dernier tour, dont la paroi supérieure est plane-inclinée, et par son ouverture oblongue, dont les bords (supérieur et columellaire) déclives-rectilignes sont presque parallèles.

I. pachestha Letourneux et Bourguignat, sp. nov.

Testa sat aperte ac profunde perforata, globoso-depressa, solidula, opa- . cula, subnitente, striatula, in ultimo malleata, albidula ant rare subal- 
bido-zonata (zonæ subrufulæ, numerosæ, sæpe interruptæ); spira parum elata, in tholum rotundata (apex rufus, exiguus); anfractibus $61 / 2$ vix convexiusculis, carinatis (carina suturam sequens), regulariter ac sat celeriter usque ad ultimum, deinde velociter, crescentibus, sutura subimpressula, in ultimo impressiore, separatis; ultimo magno, tumido-rotundato, ad aperturam perinflato ac ampliore, superne ad insertionem breviter descendente; apertura subobliqua ac parum lunata, subsphærica ac fere'æque alta ac lata; peristomate recto, acuto, tenui, intus vinoso, profunde albo-labiato, ad columellam dilatato; alt. 16 , diam. 23 millim.

Collines entre Ghardimaou et Ben-Bechir (Pechaud).

Environs de Tebessa (Let.), dans la province de Constantine.

Son dernier tour bien renflé-arrondi, relativement énorme, surmonté d'une spire en forme de dôme, fera distinguer facilement cette Hélice des précédentes, qui toutes ont une spire élancée-conoïde.

II. Charmesiana Letourneux et Bourguignat, sp. nov.

- Testa profunde angusteque perforata (perforatio semitecta), globosa, solidula, opacula, vix striatula quasi polita, albidula, ad peristoma rufolutescente; spira elato-rotundata, in tholum gibbosá, ad summum perobtusa (apex minutissimus, rufús, 'leviter prominens); anfractibús ' 7 convexiusculis, carinatis (carina suturam sequens), lente usque ad ultimum, deinde rapide crescentibus, sutura lineari, in ultimo valide impressa, separatis; ultimo magno, ventroso-rotundato, superne e dimidio evolutionis paulatim descendente; apertura obliqua, mediocriter lunata, subovato-rotundata; peristomate recto, acuto, tenui, intus vinoso-aurantiaco, profunde albo-labiato, ad columellam valide dilatato; alt. 18 , diam. 23 millim.

Sur les plantes des collines au-dessus de Ghardimaou (Pechaud).

Chez cette espèce, la direction descendante, qui commence à se faire sentir à moitié de la circonvolution du dernier tour, finit pár devenir si accentuée que la convexité de l'avant-dernier fait ventre au-dessus de sa paroi supéro-aperturale. Ce caractère, non moins que celui du bombement très prononcé de la spire, suffit pour la distinction de cette belle Hélice, à laquelle nous avons attribué le nom de M. Xavier Charmes, Directeur au Ministère de l'Instruction publique.

\section{Billottiana Letourneux et Bourguignat, sp. nov.}

Testa late aperteque perforata aut potius umbilicata (umbilicus in ultimo dilatatus), subdepresso-conoidali, nihilominus bene globosa, opacula, nitente, argutissime striatula, quasi polita, in ultimo passim malleata, uniformiter albida; spira depressula, parum elata; convexo-conoidali (apex rufus, exiguus); anfractibus 7 convexis, regulariter ac sat celeriter crescentibus, sutura subprofunda separatis; ultimo magno, ; rotundato, superne ad insertionem fere recto; apertura obliqua, Junata, semiovato- 
rotundata; peristomate recto, tenui, acuto, intus vinoso, profunde albolabiato, ad columellam mediocriter dilatato; alt. 16 , diam. 25 millim.

Collines au-dessus de Tebourba (Berthier).

Cette Hélice, dédiée à M. René Billotte, chef de bureau au Ministère de l'Instruction publique, est, parmi les formes de ce groupe, celle qui a la plus large perforation, la croissance la plus régulière, la suture la plus profonde et le dernier tour rectiligne à l'insertion, etc.

\section{S 64.}

La série de l'H. entara se compose de sept espèces [H. piratarum (Kobelt), limonorea, eucesta et cucestella (Bourg.), Derbana (Let.), entara et phana (Let. et Bourg. )], toutes des contrées nord-occidentales de l'Afrique. Ce sont de belles Hélices globuleuses-conoïdes ou subconoïlcs, médiocrement épaisses, blanchâtres plus ou moins zonulées, à ouverture semi-ronde bien daus l'axe de la coquillc, à péristome droit, aigu, faiblement bordé et à perforation ombilicale profonde plus ou moins ouverte suivant les espèces.

Ces Hélices, bieı qu’il soit difficile (saus entrer dans de minutieux détails, qui ne peuvent prendre place que dans une étude monographique) de les spécifier au point de vue de leurs caractères de groupement, possèdent entre elles un air de parenté qui ne permet pas de les répartir dans aucunes des séries xérophiliennes.

\section{I. entara Letourneux et Bourguignat, sp. nov.}

Testa aperte perforata (perforatio in centro profunda, ad ultimum leviter dilatata), globosa, opaca, nitida, valide striata (striæ in superioribus quasi costulatæ, regulares, sat producta, in ultimo rudiores ac prope aperturam teuuiores), albidulo-lutescente aut albido-zonata (zonæ rulæ, quarum una circa suturam interrupta, altera lata conlinua supera et cæteræ inferiores plus minusve numerosæ); spira subconoidali-rotundata, mediocriter producta (apex rufus, exiguus, splendens); anfractibus $61 / 2$ convexiusculis, primo regulariter deinde in ultimo rapide crescentibus, sutura lineari, ad aperturam impressula, separatis; ultimo magno, amplo, rolundato, superne sat valde descendente; apertura parum obliqua ac lunata, semirotundata; peristomate recto, acuto, intus profunde incrassatulo, leviter patulescente, ad columellam dilatato; alt. 13 , diam. 20 millim.

Environs de Feriana (Let.).

H. eucestella Bourguignat, sp. nov.

Testa profunde perforata, globosa, opaca, nitida, subtilissime striatula, fere polita, candida ac zonulis saturate rufis continuis (quarum una latior supra prope peripheriam, alteræ 4 inferiores et angustiores) cincta; spira convexo-subconoidea, ad summum obtusa (apex ater, exiguus); an- 
fractibus 6 convexiusculis, regulariter ac nihilominus rapide crescentibus, sutura impressula separatis; ultimo majore, rotundato, superne valde descendente ac ad insertionem deflexo; apertura obliqua, parum lunata, subovato-rotundata, nihilominus æque alta ac lata, intus 'splendide margaritacea cum zonulis tribus atro colore apparentibus; peristomate recto, acuto, intus profunde labiato, ad columellam dilatato; marginibus sat approximatis; alt. 14 ; diam. 21 millim.

Collines près Ghardimaou (Pechaud).

Cette Hélice se distingue de l'H. entara par son test plus épais, presque poli et d'une coloration différente, par ses tours plus convexes, à croissance spirale rapide moins régulière et séparés par une suture non linéaire, par son dernier tour un peu moins gros, excessivement descendant et défléchi à l'ouverture, ce qui rend celle-ci plus oblique avec une apparence légèrement subovale, par son encrassement intérieur du péristome plus enfoncé, etc.

Pour l'H. eucesta (Bourg.), espèce encore inédite des environs de Teniet-elHaad (province d'Alger), cette Hélice diffère de celle-ci par sa large perforation, par son dernier tour globuleux bien ventru, offrant, à l'insertion, une faible et courte direction descendante, par son ouverture moins oblique, semi-ronde, non ovalaire, bien dans l'axe de la coquille, par son péristome dont l'enerassement plus robuste est plus rapproché du bord.

\section{$\$ 65$.}

La série des H. Seetzeni (H. Seetzeni (Koch), Jabusceana, Jordanica, Judceana, Benjaminica (Bourg.) etc.), très développée en Syrie, offre dans nos possessions du nord de l'Afrique d'assez nombreux représentants spécifiques, tels que les $H$. ectata, stereolena, Dryitorum, Adisana (Bourg.), Thevestensis (Let.), occonella (Let. et Bourg.), auxquels il convient encore d'ajouter l'H. camerata (Mousson) du Maroc (1).

Sur ces sept espèces africaines, les trois suivantes ont été constatées en Tunisie.

H. occonella Letourneux et Bourguignat, sp. nov.

Testa anguste perforata, sat depressa nihilominus inflato-tumida, opacula, nitida, argute striatula quasi polita, albidula cum zonulis rufulis 3-5 angustis, sæpe subevanidis; spira convexo-rotundata, tholiformi, parum producta (apex minutus, rufus, leviter prominulus); anfractibus 6 convexiusculis, regulariter ac sat celeriter crescentibus, sutura lineari separatis; ultimo magno, rotundato-inflato, ad insertionem leviter descendente; apertura subobliqua, sat lunata, exacte hemisphærica, æque alta ac lata, intus rosacea; peristomate recto, acuto, intus valide roseolabiato et leviter patulescente, ad columellam robustam mediocriter dilatato; alt. 14 , diam. 19 millim.

(1) L'Helix Nandieri (Bourg. in Locard, 1882) de la Rivière de Gènes doit ètre également rapportée à cette série. 
Aïn-Gherichira; de Sousa à Monastir et à Mehedia; dunes entre Nebeul ef Menzel-Temim; Feriana (Let).

1. stereolena Bourguignat, sp. nov.

Testa vix puncliformi-perforata ac deviatione ultimi anfractus rimata sicut in Helix obstructa (Fer.), ventroso-pertumida, quamvis spira depressa sit, cretacea, opaca, nitida, eleganter striatula, candida cum zonulis castaneis, angustis, continuis (quarum una supera fere ad peripheriam, alteræ 5 inferiores); spira parum elata, quasï compressa, convexa (apex minutissimus, prominulus, castaneus); anfractibus 6 convexiusculis, lente usque ad ultimum, deinde rapide crescentibus, sutura impressa separatis; ultimo nagno, pertumido, maxime in altitudiuem inflato, rotundato, ad insertionem breviter subdeflexo; apertura vix obliqua, perlunata, hemisplrarica, magis alta quam lata, intus albo-margaritacea cum zonulis atro colore apparentibus; peristomate recto, obtuso, albo-incrassato, id columellam subelilatato; alt. 14 , diam. 19 millin.

Endroits arides aux envirous de Tunis (Bourg. 1867).

Espèce remarquable par l'excessif renflement de son dernier tour, par son ouverture très échancrée et surtout par sa perforation à peine sensible, se prolongeant en ligne droite par suite de la direction rectiligne du bord coluntellaire.

\section{H. Adlisana Bourguignat, sp. nov.}

Testa anguste profundeque perforata, globosa, fere sphæroidea, opacula, nitente, striata, candida cum zonulis 5-6 rufis, continuis, angustis, quarum una circa sulurim, alteræ tres medianæ et cæleræ circa perforntionem; spira sat elata valde convexil, exacte sphæroidea (apex perexiguus, rufulus, subprominulus); anfractibus 6 subconvexiusculis, regulariter crescentibus, sulura lineari, ad aperturam impressula, separatis; ullimo magno, tumido-cylindrico, ad insertionem recto; apertura vix obliqua, mediocriter lunata, hemispherica, intus albida cum zonulis apparentibus;'peristomate recto, acuto; intus labiato, ad columellam superne vix expanso; alt. 13 , diam. 16 millim.

Cetie Hélice globuleuse, ressemblant à ume petite boule, a été recueillic sur les rochérs au sud du lac El-Bahira non loin de Radès (Bourg. 1867).

\section{$\$ 66$.}

Les Hélices de cette série forment un groupe remarquable par leur coloration violette, parfois très intense lor'squ'elles sont vivantes. $\Lambda$ la longue, lorsqu'elles sont mortes, cette belle coloration finit malheureusement par disparaitre en grande partie. Toutes ces espèces violacées, de forme gobuleuse, ì lest brillant et poli, semblent spéciales aux régions chaudes du centre de la Tunisię. Nue (I'H. clithia) a été constatée en Sicile. 
1. blossura Letourneux et Bourguignat, sp. nov:

Testa profunde ac sat aperte perforata, globoso-conoidea, opacula, nitida, polita, subtiliter striatula, albido-subviolacea, aliquando in supcrioribus rufo-maculata; spira elato-conoidea, ad summum obtusiuscula (apex minutus, rufus, subprominulus); anfractibus 7 vix convexiusculis, sat celeriter. crescentibus, sutura lineari, in ultimo impressula, separatis; ultimo majore, rotundato, supra leviter subdeclivi, ad insertionem plus minusve descendente; apertura obliqua; lunata, semirotundata in directionem leviter dectivem, æque alta ac lata, intus riolaceo-lutescente; peristomate recto, acuto, intus sat profunde labiato, inferne subpatulescente, ad columellam mediocriter dilatato; alt. 16 ; diam. 19 millim.

Ain-Cherichira; environs de l'amphithéâtre d'El-Djem; dunes entre Nebeul et Menzel-Temim; de Sousa à Monastir el Mehedia (Let.).

II. elithia Leiourneux et Bourguignat, sp. nov.

Teșta anguste profundeque perforata, globosa, opacula, nițdissima', polita et argutissime striatula, uniformiter subviolaceo-lactescente, rare in prioribus rufo-flammulata, in specimine unico zonulis 8 rufis (superior'es 2 interruptæ [quarum una suturalis] et alteræ 6 [una continua, mediana ad peripheriam] plus minusve interruptæ) anguste circumcincta; spira elato-convexa, ad summum obtusa (apex minutus, rufus, prominulus); anfractibus 7 vix convexiusculis, lente usque ad ultimum, deinde rapide crescentibus, sutura lineari, in ultimo impressa, separatis; ultimo magno, rotundato, ad initium obscure subangulato, ad insertionem recto aut vix subdescendente; apertura parum obliqua ac lunata, exacte hemisplıærica, intus leviter rosaceo-violacea; peristomate recto, acuto, intus labiato, ad columellam vix dilatato; alt. 13 , diam. 18 millim.

Aïn-Cherichira; de Sousa à Monastir et à Mehedia; amphithéêtre d'El-Djem; d'El-Kef à Souk-el-Arba (Let.).

Variété minor dans les dunes entre Nebeul et Menzel-Temim (Let.).

Cette Hélice diffère de l'H. blossura par sà forme non conique, par sa spìre moins élevée, simplement convexe, par sa croissance spirale plus accélérée au dernier tour, qui est plus globulenx et presques rectiligne à l'insertion, par son ouverture plus ample, par sa perforation moins ouverte, etc.

\section{A rbana Letourneux et Bourguignat, sp. nov.}

Testa profunde angusteque perforata. (perforatio leviter angustior), globoso-subconoidea, leviter translucida, tenuiore, nitida, subpolita, argute striatula, albido-sublutescente aut pallide subcæruleo-violacea, ad superiores rufo-flammulata; spira elata, obtuse conoidea, ad summum obtusa (apex minutus, rufus, non prominulus); anfractibus 7 convexiusculis, lente usque ad ultimum, deinde rapidius crescentibus, sutura im- 
pressula separatis; ultimo magno, exacte cylindrico, ad insertionem recto; apertura parum obliqua et lunata, ampla, hemisphærica, magis alta quam lata, intus obscure subviolaceo-lutescente; peristomate recto, acute cultrato, intus mediocriter incrassato, ad columellam dilatato; alt. 14 , diam. 18 millim.

Entre El-Kef et Souk-el-Arba et sur la frontière tunisienne entre Haïdra et Tebessa (Let.).

Cette espèce se distingue de la précédente par son test plus délicat, assez transparent, par sa spire plus élevée, sensiblement conoïde; dont le sommet n'est pas proéminent, par sa perforation un peu plus étroite, par son dernier tour plus régulièrement cylindrique, par son ouverture plus ample, dont la hauteur dépasse la largeur (chez l'H. elithia, l'ouverture n'est pas plus haute que large), par son péristome mince, tranchant et offrant un bourrelet médiocre, non marginal comme celui de l'H. elithia, mais relativement assez enfoncé dans l'intérieur.

\section{Bonstoma Letourneux et Bourguignal, sp. nov.}

Testa anguste perforata (perforatio parum profunda in ultimo leviter dilatata), globosa, obtuse conoidea, leviter subtranslucida, nitida, striatula, in ultimo submalleata, uniformiter albido-violacea; spira elatoobtusa, subconoidea (apex minutus, rufus, non prominulus); anfractibus 7 vix convexiusculis, regulariter ac celeriter crescentibus, sutura lineari, in ultimo impressa separatis; ultimo magno, rotundato, superne perlente subdescendente; apertura parum obliqua ac lunata, subsemicircnlari, magis alta quam lata, intus saturate castaneo-violacea; peristomate recto, acuto, intus plus minusve labiato, ad columellam dilatato; alt. 17 , diam. 21 millim.

Eutre El-Djem et Kerouan (Let.).

La forme de sa perforation, son test d'une belle coloration blanche-violacée, son intérieur apertural d'un marron violet foncé, non ınoins que sa tailte plus forte, etc., feront distinguer cette espèce des précédentes.

B. ianthinostoma Letourneux et Bourguignat, sp. nov.

Testa perforata (perforatio profunda, in ultimo dilatata), globoso-depressa, solida, opaca, nitida, polita, subtilissime striatula, uniformiter pallide violacea, in specimine unico subviolaceo-lutescente, cum zonulis castaneis fere evanescentibus; spira sat depressa, convexa (apex minutus, rufo-violaceus, non prominulus); anfractibus 7 vix subconvexiusculis seu potius subplanulato-tectiformibus, celeriter crescentibus, sutura lineari, in ultimo impressula, separatis; ultimo magno, rotundato-inflato, ad insertionem breviter subdescendente; apertura obliqua, lunata, semicirculari, aæque alta ac lata, intus sálurate violacea; peristomate recto, leviter obtuso, incrassato, inferne patulo, ad colunellam dilatato; alt. 17 , dianı. 23 millim. 
Entre El-Djem et Kerouan (Let.).

Cette Hélice diffère de l'II. ionstoma par son test épais, opaque, d'une teinte plus violacée, par sa forme non conoïde, mais déprimée, par ses tours plus plans, par son ouverture plus oblique, pas plus haute que large, d'un beau violet foncé dans l'intérieur, entourée d'un bord péristomal obtus.

\section{H. amethysta Letourneux et Bourguignat, sp. nov.}

Testa anguste profundeque perforata, pertumido-globosa, fere sphæroidea, opacula, nitida, striatula, albo-violacea cum zonulis castaneis, sæpe evanidis ac confluentibus et plus minusve ( 2 ad 12 ) numerosis; spira in tholum rotundata (apex exiguus, rufus, subprominulus); anfractibus 7 convexiusculis, celeriter (in ultimo rapidius) crescentibus, sutura lineari, in ultimo impressa, separatis; ultimo maximo, perinflato-tumido, rotundato-globoso, superne descendente; apertura parum obliqua ac lunata, exacte semicirculari, æque alta ac lata, intus saturate vinoso-violacea cum zonulis apparentibus; peristomate recto, cultrato, intus mediocriter incrassato, ad columellam vix dilatato; alt. 17, diam. 21 millim.

Entre El-Djem et Kerouan (Let.).

Cette espèce, la plus globuleuse du groupe, est très remarquable par sa belle coloration violette, sur laquelle se détachent des bandes marron du plus bel effet et par sa gorge aperturale d'un violet-vineux très foncé.

$\$ 67$.

Le docteur Servain a, le premier, distingué scientifiquement, chez l'H. pisanu, des formes spécifiques. M. Henri Berthier a suivi cet exemple. Depuis, plusieurs auteurs ont cru, avec raison, qu'il était nécessaire d'éliminer de cet amalgame d'Hélices, sous l'appellation d'H. pisana, les formes nettement caractérisées par trois signes distinctifs nets et constants; c'est également notre avis, et, à cet effet, après une étude approfondie des pisaniennes, nous sommes arrivé à admettre, pour le système européen, vingt-quatre formes ${ }^{(1)}$, qui peuvent être ainsi réparties :

$1^{\circ}$ Spire élevée, conoide, à croissance spirale régulière peu rapide. Dernier tour arrondi, de taille médiocre. Ouverture relativement petite, à péristome mince et fort peu encrassé.

Helix Couturieri, Bourg. - Algérie.

Helix Byrsæ, Marès. - Tunisie.

Helix Chambardi, Let. - Égypte, Tunisie, Italie, Istrie.

Helix thinophila, Bourg. - Egypte.

Cette dernière espèce a autant de tendance à se rapprocher de l'H. etama, Hélice du groupe de l'H. acompsia (voir ci-dessus, page 64) que de l'H. Josaphatica (Bourg.), forme du groupe syrien de l'H. Cedronica.

(i) Si on y comprend celles du pays Çomalis (voir Moll. terr. et fluv. du pays Comalis, in Mission Revoil, Paris, in-8 ${ }^{\circ}, 188_{2}$ ). 
$2^{\circ}$ Dernier tour toujours caréné ou subanguleux. Cette série doit être divisée :

A. En espèces à spire tectiforme ou convexe-arrondie, à croissance spiralo régutlière; faiblement rapide et à :ouverture d'une ampleur médiocre.

Helix Donatii, Berthier. - Italie, Espagne, Tunisie, Syrie.

Helix Cuttati, Bourg. - Espagne, Provence, Istrie:

Helix Monroi, Servain. - Espagne, Provence.

B. En espc̀ces à spire très déprimée, à croissance spirale rapide, au dernier toup trest of à ouverture bien dévcloppée.

Helix pisanella, Servain. - Espagne, Baléares, Provence, Algérie et Tunisie.

Helix Levesquei, Berthier. - Algérie et Tunisie.

Helix Salemensis, Let. et Bourg. - Tunisie.

Helix Gergisensis, Let. et Bourg. — Tunisie.

$3^{\circ}$ Dernier tour arrondi très volumineux. Spire déprimée à croissance spirale rapide. Ouverture très ample à péristome très bordé et fortement patulescent ${ }^{(1)}$.

Ilelix subpisana, Bourg. - Tunisic, Espagrne, Baléares, Istrie.

Helix Agaroi, Servain. - Espagne.

Helix Olivaresi, Servain. - Espagne.

$4^{\circ}$ Coquille globuleuse ou sphéroïde, ì test relalivement épais. Dernier tour rond. Ouvertmre plus ou unoins circulaire, à péristome fortement bordé.

Helix pisanopsis, Servain. - Portugal.

Le docteur Kobelt, sous le nom d'Helix pisanopsis, var. Eguse, a décrit (Natur. Sicil. II, 1883, p. 260) une forme sicilienne de l'ile Marctimo, qui u'a pas de rapport avec cette Hélice. C'est une espèce globuleuse du groupe des II. Grannonensis, maritima, fadata, etc.

Helix Dermoi, Servain. - Espagone, Maroc, Tunisie.

Helix Radesiama, Marès. - Tunisic, Maroc.

Helix Carpiensis, Let. et Bourg. - Tunisic, Syrie.

Helix Hamadanica, Let. et Bourg. - Tunisie, Chypre.

Helix lenoleuca, Bourg. - Égypte, Syrie.

$5^{\circ}$ Coquille pesante, épaisse, de forme globuleuse-turbinée, à dernier tour très grand.

Ilelix Djerbanica, Let. et Bourg. - Tunisie.

Helix Zitanensis, Let. et Bourg. - Tunisie.

Ces deux dernières espèces forment le passage entre les pisaniennes et les espèces égyptiennes du groupe des II. Forskali, Eltrenbergoi, etc.

En résumé, pour faire comprendre en quelques mots cette classification, on pourrait encore distinguer les formes pisaniennes en :

Ver.e. - H. pisana. (Type et variétés.)

Turbivate. - H. Couturieri, Byr's $x$, Chambardi et thinophila.

(1) C'est à cette série qu'apparticunent les II. Comaliana et Tohenica (Bourfr.) du pays Çomalis. 
Carinate. - H. Donatii, Cuttati, Monroi, pisanella, Levesquei, Salemensis et Gergisensis.

Depress.e. - H. subpisana, Agaroi, Olivaresi.

Grobosæ. - H. pisanopsis, Dermoi, Radesiana, Carpiensis, Hamadanica et lenoleuca.

Ponderose. - H. Djerbanica et Zitanensis.

I. pisana Müller, Verm. Hist. 1I, p. 60, 1774. (H. rhodostoma Draparnaud, 1801 et 1805).

Cette espèce a été exactement représentée dans la Malacologie de l'Algérie (I, 1884, pl. XXVI, f. 1-10). Ces figures suffisent à la connaissance de celte Hélice.

L'H. pisana est une forme essentiellement circumméditerranéenne; elle se trouve partout en excessive abondance. Dans le nord de l'Afrique, notamment en Tunisie, elle pullule, sur les plantes et les arbrisseaux, dans les vallées chaudes et abritées; elle devient moins commune dans les contrées montueuses.

La coloration de cette Hélice varie à l'infini. Les auteur's ont attribué à ces variations des désignations multiples, telles que sardoa, ferruginea, punctella, subsonata, taniola, bifrons, luteo-zonata, diaplana, concolor, roseo-albida, ulbinos, egregia, festiva, decora, multifaria, nebulosa, pustulosa, agreabilis, mcesta, clegrans, disjuncta, maculosa, etc., qui n'ont aucune importance.

Cette espèce offre encore des variations dans sa taille, dans sa croissance spirale, ainsi que dans la forme de l'ouverture; mais ces dernières variations moins nombreuses, restreintes du reste dans certaines limites, ne sont pas accentuées au point de dénaturer ses signes distinctifs. Parmi ces variations nous n'en voyons qu'une en Tunisie qui soit digne d'être signalée; nous l'avions primitivement désignée sous l'appellation d'Helix Thusurosi.

G'est une variété de petite taille ( $H .9, D .13$ millim.), à perforation étroite peu profonde, à test mince, transparent, finement striolé, blanchâtre, à spire déprimée peu convexe, à cinq tours de croissance rapide, dont le dernier très grand, comprimé-subanguleux à l'origine, rectiligne à l'insertion, ample-arrondi ver's les bords marginaux, offre une ouverture verticale, échancrée, ovalaire-arrondie, entourée par un péristome droit, aigu et peu bordé.

Cette variété, recueillie aux environs de Tozer (Let.), a encore été constatée à Cascaẽs en Portugal et à Beyrouth en Syrie.

\section{Byrsae Marès, sp. nov.}

Testa sat parvula, anguste perforata, globulosa, subconoidea, teuri, subtranslucida, parum nitente, subtiliter striatula cum lineis spiralibus minutissimis vix sub lente perspicuis, candida aut luteola vel subcarneola cum zonulis duabus inferis, atris continuisque, rarissime cum zonulis 5 quarum superiores 3 eleganter interruptæ; spira elato-conoidea, ad summum oblusa (apex nigrescens); anfractibus 6 convexiusculis, regulariter crescentibus, sutura impressula separatis; ultimo majore, nihilominus relative mediocri, rotundato, superne recto vel lente descendente; aper-

Malacologie. 
tura parum obliqua, lunata, semirolundata aut subrolundo-ovata, intus albida, rosacea aut luteola; peristomate rare rosaceo, recto, aculo, sat tenui, intus leviter incrassatulo, inferne patulo ac ad columellam dilatato et supra perforationem panlulum reflexo; alt. 13 , diam. 15 millim.

Environs de Radès, près de l'embouehure de l'Oued Meliana entre Tunis et Hammam-el-Lif (Narès); ruines de Carthage (Peehaud).

H. Clnambardi Letourneux, sp. nov. 1877 .

Testa minuta, profunde ac plus minusve aperte perforala, conoidea, subopacula, mediocriter nitente, striatula cum lineis spiralibus vix perspicuis, uniformiter albida aut lutescente cum zomulis 7-8 continuis, subcastaneis, fere evanido-oleatis; spira elato-conoidea, ad summum obtusinscula (apex nilidus, sæpe rufulus aul ater); anfractibus 6-7 convexiusculis, regulariter crescentibus, sutura impressa separatis; uliimo inediocri, subcompresso-rolundato, supra leviter lectiformi-declivi, superne ad insertionem perlente descendente; apertura parum obliqua, lunata, transverse rotundato-subovata, intus albido-margaritacea (in speeimine pedemontano rosacea, in aliis istrianis pallide castanea cum zonnlis apparentibus); peristomate recto, inlus profunde incrassalulo, inlerne patulescente et ad colımellan mediocriter dilatato; alt. 10 , diam. 11 millim.

La forme typique de eette espèce, découverte en Kigyple dans les dunes de Ramleh, près Alexaudrie, n'a pas été retrouvée en Tunisie, mais seulement une variété maxima (haut. 16-19, diam. 20-22 millim.) aux environs de Radès (Marès).

Cette 11. Chambardi, bien que variant beaueoup eomme taille et eomme eoloration, reste néanmoins constante dans les signes cardetéristiques que nous venons d'indiquer.

\section{Donatii Berthier, sp. nov.}

Testa angustissime perforatil (perloratio omnino tecta), depresso-tectiformi, supra conoidea, sublus convexa, angulala aul plus minusve eatrinala, opacula, nitente, argute striatula (in ultimo malleala) cum lineis spiralibus bene perspicuis, uniformiler candida; spira eonoidea, sat elata, tectiformi, ad summum obtusiuscula (apex splendens, sat robustus); anfractibus 6 vix convexiuseulis, regulariter crescentibus, sutura lineari (ad aperturam impressula) separatis; ultimo majore, mediane carinato (carina plus minusve acuta, ad aperturam evanescens), supra subconvexo-declivi, subtus convexiore, ad insertionem leviter descendente; apertura vix obliqua, mediocriter lunata, semiovata, in directionem leviter transversalem declivi, aliquando in margine externo subangulata, intus pallide rosacea; peristomate reclo, acuto, intus incrassato, inferne vix patulescente et ad columellam superne late dilatato-reflexo; alt. 14 , diam. 18 millim. 
Environs de Tozer (Let.).

Le type de cette Hélice se trouve en Syrie aux euvirons de Jaffa. Les échantillons que nous connaissons d'Italie (le Lido près Venise) et d'Espagne (Cadix), sont mieux caractérisés que ceux de Tunisie.

\section{I. pisanella Servain, Moll. Esp. p. 113 (sine desc.) 1880.}

Testa rix punctiformi-perforata, carinata, supra depressa, parum convexa; subtus convexiore, opacula, nitida, subtiliter striatula cum lineis spiralibus bene notatis, albida aut lutescente et rare cum zonulis rufis plus minusie numerosis; spira depressa, parum elata, convexa aut tectiformi (in speciminibus massiliensibus fere subplanulata), ad summum perobtusa (apex exiguus, lucidus, lævigatus); anfractibus 5 vix convexiusculis, velociter crescentibus, sutura lineari separatis; ultimo magno, carinato (carina ad aperturam evanescens), supra declivi-subconvexo, subtus convexiore, ad aperturam subrotundato, ad insertionem recto aut breviter deflexo; apertura fere verlicali, lunata, semirotundata, intus albidula aut subrosacea; peristomate recto, acuto, breviter labiato, ad columellam superne modo dilatato-reflexo; alt. 10-11, diam. 15-18 millim.

L'H. pisanella ${ }^{(1)}$ parait assez abondante en Espagne aux environs de Valence, de Séville, etc., ainsi qu'aux Baléares; en Algérie, elle a été constatée bien typique dans la vallée de la Seybouse près Bône; enfin, en Tunisie, elle a été recueillie sur les dunes littorales entre Sfax et Gabès (Berthier).

\section{H. Levesquei Berthier, sp. nov.}

Testa vix punctiformi-perforata (perforatio tecta, rimæ minutissimæ similis), carinata, supra fere planulata, subtus tumido-convexa, sat cretacea, opaca, non nitente, striata (in ultimo passim malleata) cum lineis spiralibus bene adnotatis, uniformiter candida; spira omnino compressa, vix convexiuscula (apex albus, minutus); anfractibus $4-5$ sat convexis, rapide crescentibus, sutura impressa, fere profunda, separalis; ultimo maximo, fere totam altitudinem æquante, carinato (carina ad aperturam subevanida), supra prope aperturam turgido ac suturam superante, subtus inflato-convexo, ad insertionem recto; apertura subolliqua, transverse angusta (diam. 5 millim.), in altitudinem (alt. 10 millim.) valde ampla, intus albo-margaritacea; peristomate recto, acuto, incrassatulo, ad columellan superne modo in triangulam formam dilatalo ac supra perforationem adspresso; callo subrosaceo; alt. 10 1/2, diam. 16 millim.

Cette belle et singulière espèce a été découverte sur les dunes au sud de Gabès (Berthier).

(I) En France, cette Hélice se trouve à la Sainte-Baume (Var) et près de Marseille. 
I. Salemensis Letouneux et Bourguignat, sp. nov.

Testa anguste ac semitecte perforata, angulata, depressa, supra mediocriter convexa, subtus convexiore, solida, sat translucida, nitida, bene striata et lineis spiralibus pernumerosis valde impressis et notatis eleganter decussata, griseo-rosacea ac in paucis speciminibus zonulis rufis interruptis perangustisque cincta; spira depressa, parum convexa (apex aterriuus); anfractibus 5 convexis, rapide crescentibus, sutura impressa separatis; ultimo maximo, angulato (angulus ad aperturam evanescens), supra convexo, subtus inflato-convexiore, ad perforationem leviter turgescente, superne breviter deflexo; apertura subverticali, lunata, irregulariter transrerse subhemispherica, ad basin marginis columellaris subangulata, intus luteola aut pallide carneola; margine columellari obligue recte descendente; peristomate recto, acuto, incrassatulo, ad columellam dilatato-reflexo; alt. 11 , diam. 17 millim.

Sidi-Salem près Hount-Souk dans lîle de Djerba; environs de Zarzis (Let.).

\section{Gergisensis Letoumeux ef Bourguignat, sp. nov.}

Testa vix perforata, obscure angulafa globoso-pertumida ac nihiloininus supra depressa, solida, subopacula, nitida, bene striata ac lineis spiralibus pernumerosis, impressis sicut in præcedente, eleganter decussata, unilormiter albidula aut griseo-subrosacea, in specimine unico flammulis rufis in zonulas dispositis maculata; spira depressa, parum convexa (apex albidulus aut griseus, nilidissinus); anfractibus 5 convexiusculis, rapide crescentibus, sutura impressa separatis; ultimo maximo, inflatopertumido, cylindrico, ad initium angulato (angulus sat obsoletus, ad aperturam evanescens), superne ad insertionem recto; apertura fere verticali, lunata, ampla, semicylindrica, magis alta quam lata, intus luteola aut carneola; peristomate recto, acuto, labiato, inferne patulo, ad columellam dilatato-reflexo; alt. 13 , diam. 18 millim.

Environs de Zarzis et dans lî̀le de Djerba aux alentours d'Houmt-Souk (Let.).

Cette espèce se distingue de la précédente, la seule avec laquelle elle puisse être confondue, par sa forme excessivement ventrue-globuleuse au dernier tour, par son ouverture d'une graude amplem, plus haute que large et exactement semicirculaire; celle de l'H. Salcmensis, de forme subhémisphérique dans le sens transversal, est, au contraire, plus large que haute. Chez cette espèce, en outre, le dernier tour, déprimé, plus anguleux, est bien moins ventru.

\section{H. subpisana Bourguignat, sp. nov. 1880 .}

Testa anguste perforata, pertumido-ventrosa, supra parum convexa, cretacea, solida, opacula, robuste striata (stria irregulares, sulcis inlerruptæ), ac quasi rugulosa, uniformiter albidula, in speciminibus balea- 
ricis ochracea; spira vix convexa, in tholum rotundata (apex minutissimus, ater); anfractibus 6 subconvexiusculis rapide crescentibus, sutura lineari separatis; ultimo maximo, rotundato, amplo, ad aperturam tumido-inflato, subtus circa perforationem turgidulo, superne ad insertionen recto; apertura subverticali, lunata, semicirculari, intus pallide subrosacea; peristomate recto, acuto, valide labiato, undique patulo, ad columellam dilatato; alt. 14 , diam. 20 millim.

Environs de Tunis (Bourg. 1867).

Gette Hélice a encore été constalée en Espagne près de Séville et aux Baléares dans l'ile Minorque.

II. Dermoi Servain, sp. nov. 1884.

Comme forme, cette nouvelle espèce ressemble beaucoup à cette variélé marocaine figurée par M. Morelet (Moll. Maroc, pl. I, f. 2 seulement [la figure $2 a$ se rapporte à l'Hélice suivante], 1880 ).

L'H. Dermoi est une corguille globuleuse à spire élevée, obtuse au sommet, à test assez épais, bien brillant, très fortement décussé par des striations el des lignes spirales, qui en s'entre-croisant, forment un treillis très accusé. Chez cette espèce, la croissance spirale est régulière, les cinq à six tours sont convexes, le dernier, bien qu'un tant soit peu déprimé près de la base aperturale, est arrondi; l'ouverture, transversalement subovale-arrondie, est entourée d'un péristome robuste bien bordé et franchement patulescent. (Haut. 12, diam. 15 millim.)

Cette Hélice varie beaucoup comme coloration. An Maroc, daus la vallée de l'Oued Tydsi, à 24 kilomètres au sud de Mogador, elle est blanchâtre ou d'un fanve pâle avec deux larges loandes hrunes recouvrant presque toute la surface; en Espagne, près de Séville, où se trouve le type, elle est jaunâtre avec huit zonules très étroites, dont la supérieure senle est flammulée le long de la suture; en Tunisie, où elle a été découverte, près de Radès, par le docteur Marès, elle est d'nu jaune-rosacé pâle avec dix bandes étroites, loutes continues, de deux couleurs: sept sont d'un marron rougeâtre et les trois autres (celle de la périphérie et les deux plus rapprochées de la perforation) sont d'un bean noir foncé.

H. Radesiana Marès, sp. nov. 1884.

Cette belle espèce, également recueillie aux alentours de Radès, entre Tunis et Hammam-el-Lif (Marès), est cette autre Hélice considérée encore par M. Morele t comme une variété de l'H. pisana et représentée comme telle dáns ses Mollusques du Maroc (p. 23 , pl. I, f. $2 a$ [seulement], 1880).

Cette $H$. Radesiana, espèce assez répandue au sud du Maroc, est une coquille (haut. 12-16, diam. 13-18 millim.) lout à fait globuleuse-sphéroïde, à ouverture semi-circulaire entourée d'un bord péristomal robuste, bien bordé et nettement patulescent; sa spire est élevée; sa croissance spirale est rapide bien que régulière; ses tours, au nombre de six, sont médiocrement convexes, sauf le dernier, qui, en outre, est brièvement défléclii à l'insertion. 
11. Carpiensis Letourneux et Bourguignal, sp. nov.

Testa anguste perforata, globosa, subsphæroidea, solida, opaca, nitida, decussata (striæ et lineæ spirales argutæ), quasi polita, nihilominus in ultimo malleata, uniformiter sordide albidula aut albo-subcærulescente cum zonulis duabus inferis, nigris ac continuis; spira producta, obtuse convexo-rolundata (apex minutus, aterrimus); anfractibus 6 convexiusculis, regulariter crescentibus, sutura aut lineari aut impressula separatis; ultimo magno, cyliudrico, superne subrecto; apertura vix obliqua, lunata semirotundata in directionem leviter transversalem, intus lutescente; peristomate recto, acuto, intus robuste labiato, patulescente, ad columellam late dilatato-reflexo; ilt. 12 , diam. 15 millim.

Collines près d'Hammam-el-Lif (Let.). Cette espèce a encore été constatée en Syrie près de Beyrouth.

L'II. Carpiensis diffère de l'H. Radesiana par sa forme moins renflée-sphéroüde, par son test presque poli sur lequel les striations et les lignes spirales sont peu perceptibles, par sa spire moins élevée, par son dernier tour moins ventru, recliligne supérieurement et non brièvement délléchi à l'insertion, par son ouverture moius exactement circulaire, etc.

\section{Hamadaniea Letourneux el Bourguignat, sp. nov.}

Testa plus minusve anguste perforata, globosa nihilominus supra relalive medioeriter producta, solidula, teviter opacula, nitida, argute striatula et lineis spiralibus minulissime decussata, uniformiter sordide albidula, aut rosea, aut luteola cum zonulis duabus medianis ad peripheriam quarum una suecinea, continua, et altera aterrime punclulata; spira convexa, mediocriter elata, perobtusa (apex minulus, rufus, nitidissimus); anfractibus 5 convexiuscnlis, regulariter crescentibus, sutura impressula separatis; ultimo maximo, ad initium obscure subangulato ac supra convexo el sublus in directionem subplanulato-declivem attenuato, ad aperturam lumido-inflato, rotundato, inferne circa perforationem turgidulo, superne inl insertionem recto ac breviter deflexo; apertura subverticali, lunata, hemisphærica, leviter magis alta quam lata, ad basin obscure subangulata, inlus pallide luteola; peristomate recto, acuto, intus incrassatulo, ad columellam triangulatim superne dilatato; alt. 12, diam. 14 millim.

Région des Hamada; environs d'Hammam-el-Lif et de Gabès (Let.).

Cette Hélice existe également dans l'île de Chypre à Famagousle.

\section{Djerbanica Letourneux et Bourguignat, sp. nov.}

Testa minute vix perforala, obscure subangulata nihilominus globosa, supra convexo-tectiformi, subtus inflato-convexiore, solida, opaca, ponderosa, nitente, subtilissime decussata ac passim malleata, uniformiter sor- 
dide luteola aut albido-subrosacea; spira tectiformi-convexa (apex griseus); anfractibus 5-6 convexiusculis, valde carinatis (carina suturam sequens et aliquando leviter superans), regulariter celeriterque crescentibus, sutura impressula separatis, ultimo maximo, subangulato (angulus usque ad peristoma perspicuus), ad initium leviter depresso, ad aperturam inflatorotundato; apertura subobliqua, valde lunata, semirotundata leviter in transversalem directionem, magis lata quam alta, intus in callo rosacea et ad partem columellæ superam luteola; peristomate recto, acuto, intus incrassatulo, ad columellam dilatato; alt. 13-1 7, diam. 17-21 millim.

Houmt-Souk dans l'île de Djerba; environs de Zarzis et région des Hamada (Let.).

I. Zitanensis Letourneux et Bourguignat, sp. nov.

Testa punctiformi-perforata, globoso-tumida, solida, ponderosa, nitente, subtilissime decussata, passim malleata et prope aperturam hur: illuc leviter gibberosa, uniformiter albido-subcarneola; spira convexa, rotundata (apex pallide subrufulus); anfractibus 5 convexiusculis, regulariter crescentibus, sutura impressula separatis; ultimo rotundato, prope peristoma peramplo ac in buccinam dilatato, superne ad insertionem recto aut potius leviter ascendente; apertura subobliqua, lunata, semirotundata, magis alta quam lata, ad margines effusa, intus margaritacea, in pariete penultimi convexitatis modo rosacea; peristomate recto, cultrato, intus incrassato; margine columellari recto, triangulatim dilatato ac ad basin acute attenuato; alt. 15 , diam. 17 millim.

Celte singulière Hélice, remarquable par sa grande ouverture évasée, par sa coquille épaisse, solide et pesante, vit sur la colline de Medinet-Zian, près de Zarzis (Let.).

$\$ 68$.

On pourrait s'étonner de nous voir placer immédiatement après le grroupe des $H$. pisana celui si différent de l'H. Doumeti, si nous n'avertissions pas qu'entre ces deux groupes plusieurs séries de formes intermédiaires manquent en Tunisie, séries qui nous auraient conduit insensiblement, si elles eussent existé, par la filière des II. Dehnei, subdentata, erythrostoma, planata, Laurenti, Gualtieriana, etc., aux espèces que nous allons signaler.

Les Hélices dı groupe de l'H. Doumeti, toutes du nord occidental de l'Afı'ique , sont des coquilles écrasées, lentiformes, à carène aiguë et à large ombilic en entonnoir. Sans compter les espèces tunisiennes (II. Doumeti, enica, idia), ce groupe comprend encore l'H. depressula (Parreyss) d'Algérie (1), les H. Murustagensis et calladiensis (Pechaud) de la province d'Oran, l'H. alrana (Let.) de Kabylie, l'H.

(1) Le type vit près de Mostaghanem, où il a été découvert par le docteur Wagner qui l'a communiqué à Parreyss. 
subscabriuscula (Bonrg.) (1), du Maroc; enfin, l'H. explanata (Müller) qui s'étend depuis Algger, en snivant les côtes du littoral hispanique, jusqu’à Marseille.

1. Doumeti Bourguignat, Spec. nov. Moll. I, $1876, \mathrm{n}^{\circ} 48$. (Helix Lacosteana Morlel, in Arch. miss. scient., 1881 , p. 394 , pl. VI, fig. 1-2.)

Il convient de rapporter encore à cette Hélice celle que M. le professeur Isse] (Moll. croc. Viol. in Mus. civ. Genova, 1880, p. 271) a désiơnée sous l'appellation d'H. depressula (non Parreyss) et quil signale comme trouvée par Belucci, en 1875 , sur le versant sud de l'Aïd Udi, mieux Djebel Aïdoudi (au nord dn choll el-Fedjedj). Or, cette localité est l'endroit où M. André, en 1878 , a recueilli l'Lelix Lacosteana, qui n'est autre que l'H. Doumeti.

Le type de l'H. Doumeti a été trouvé, en 1874 , par M. Doumet-Adanson dans lc Djebel Bou-Hedma ${ }^{(2)}$, entrc Gafsa et Sfax. Depuis ce savant voyageur, cette Hélice a étć recucillic en très grande abondance dans presque tout le sud de la région montueuse de la Régence, à Feriana, Guclaat-es-Snan, Khanget-el-Slougui, Tamesmida, Foum-el-Goubel et Foum Bouibet, Aïn-Cherichira, Djebel Seftimi, daus les vallées de l'Oued Cherb et de l'Oued Taferma, etc. (Let.).

\section{H. enica Letourneux et Bourguignat, sp. nov.}

Testa late pervieque umbilicata (unbilicus infundibuliformis), complanato-leuticulari, acute bicarinata (carina una ad peripheriam compressa cultrata, altera circa umbilicum), supra convexiuscula, solidula, opacula, sordide albidnla aut griseo-cinerea cum zonulis rufo-violaceis 3-4 inferis, contimnis, parum saturatis, valide costulala sive rugosa (costæ robustæ, producla, obliqux, inæquales, tum validiores tum tenuiores ac sæpe præsertim sublus non continuæ); spira valde depressa, vix convexiuscula (apex nilidus, levigalus, minutus); anfraclibus 5 percarinatis (carina suturam sequens, rare superans), supra subconvexiusculis (convexitas prope carinam compressa, planulata ac sulcum simulans), regulariter crescentibus et sutura lincari separatis; ultimo majore nihilominus mediocri, acute bicarinato (carina peripherialis peracute cultrata, compressa sicut alæformis, fimbriata, intus in fauce aperturæ canaliculum simulans : carina umbilicalis acuta, xqualiter fimbriata), supra convexiusculo, ad insertionem recto, subtus e carina supera ad inferam recte declivi; apertura obliqua, irregulariter subtetragona, in margine externo profunde canaliculata, intıs albida cum zonulis apparentibus; peristomate recto, acuto, vix incrassatulo; alt. 5, diam. 17 millim.

Oued-el-Hammam au sud du Djebel Zaghouan (Let.).

(i) Helix subscabriuscula Bourg. Nalac. Alg. II, p. 320, 1864 (Helix scabriuscula [non Des. hayes] Morelet, in Journ. Conch. 1853, p. 40, et IIelix sultana Morelet, Moll. Maroc, p. 31, pl. Il, 1880).

(2) C'est par suite d'unc audition inexactc du nom dc celle localité, que l'on a indiqué Djebut! Illmar au liel de Bou-Hedma dans la description de cette llélice. 
II. Idia Letourneux et Bourguignat, sp. nov.

Testa late infundibuliformi-umbilicata, supra omnino complanata, imo sæpe concaviuscula, acute bicarinata (carina una peripherialis compressa, fimbriata, altera circa umbilicum producta, subcrenulata, sulco contracto sive funiculo similis), solidula, opacula, sordide grisea, costulata (costulæ albidulæ sicut in præcedente, sed tenuiores ac subtiliores); spira omnino planata (apex minutissimus, nitidus ac lævigatus); anfractibus 5 percarinatis (carina semper prominens ac leviter suturam linearem superans), supra planatis, ad carinam sulco impressis, celeriter crescentibus; ultimo magno, acute bicarinato (carina peripherialis sicut in præcedente, sed carina circa umbilicum producta, subcrenulata ac lyratiformis), subtus e carina supera ad inferam oblique declivi; apertura obliqua, tetragona, bicanaliculata (canaliculus unus, inferus; alter in margine externo), intus grisea; peristomate recto, acuto, simplici; alt. 4, diam. 13 millinn.

Sous les pierres au Djebel Reças (Let.) et sur les collines près d'Hammam-el-Lif (Berthier).

Cette singulière Hélice se distingue de la précédente par sa taille moindre, par son test plus finement costulé, par sa spire complètement plane en dessus, par sa croissance spirale plus rapide, par sa carène circumombilicale plus forte du double, formant saillie et ressemblant à un bourrelet crénelé, par son ouverture exactement tétragone, plus étroite dans le sens transversal et bicanaliculée.

Celte Hélice, de même que l'Il. enica, ne peut être confondue avec l'H. tetragona, attendu que celle-ci est une forme de la série de l'H. mograbina, caractérisée, comme toutes celles de cette série, par un test non costulé, mais tuberculeux.

\section{§ 69.}

Les Hélices de ce groupe (groupe de l'II. Gargottce) sont presque toutes (H. Gargottee Philippi, praclara Cafici, chonomphala Bourg.) de Sicile, ou (H. Spratti Pfeiffer, Schembrii Schwerzenbach) de lî̀le de Malte. Deux seulement semblent spéciales au nord de l'Afrique: l'une, des environs de Nemours, de Beni-Saf et de l'île Rachgoun dans la province d'Oran, est cette Hélice que M. J. Pechaud arait primitivement dédiée à M. Joly d'Alger ( $H$. Jolyana) et qu'un auteur allemand a désignée sous la nouvelle appellation de Sigensis, qui ne lui convient nullement, puisque cette espèce ne se trouve pas dans la localité qu'indique son nom; l'autre est l'espèce tunisienne suivante :

\section{H. Sageti Bourguignat, sp. nov.}

Testa profunde aperteque umbilicata, parvula, depressa, subangulata, supra convexiuscula, subtus convexiore, cretacea, opaca, sordide rufula cum costis albidis ac inferne cum zonulis 2 magis saturatis et parum perspicuis, valde costata (costæ robustæ, crassæ, productæ, in ultimo modo irregulares); spira mediocriter convexa (apex nitidus, lævigatus, 
atro-ruber); anfractibus 5 convexinsculis, regulariter crescentibus, sutura impressula separatis; ultimo majore, subangulato (angutus ad aperturam leviter subevanescens), supra convexiusculo et ad insertionem lente descendente, subtus bene convexo, ad aperturam rofundato; apertura obliqua, parum lunata, semirotunda, intus grisea; peristomate recto, acuto, intus mediocriter labiato, ad columellam vix dilatato; alt. $51 / 2$, dian. 9 millin.

Revers des fossés au Bardo (Bourg. 1867).

$\$ 70$.

Toutes les espèces de ce joli gronpe appartiennent au nord de l'Afrique. Ce sont les $H$. cantinica ${ }^{(1)}$ et omalestha Bourg., illibata Parreyss, illibatiformis Ancey, Audubaliana, Arsenurica, Gilbana, Tipazensis, encera et chola Pechand. Cette dernière seule a ćté constatée en Tunisie.

\section{H. ehola Pechaud, sp. nov,}

Tesla parvula, profunde umbilicata (umbilicus mediocris subpervius), depressa, carinata, supra convexo-tectiformi, subtus convexa, solidula, oplacula, non nitente, sordide cinerea cum zonulis inferis vix perspicuis, subtiliter striata; spira parum convexa, tectiformi (apex obtusus, maguus, lavigatus, nilidus); anfractibus $4 \frac{1}{2}$ leviter convexiusculis, carinatis (carina impressulam suturam sequens), lente usque ad ultinum, deinde rapide crescentibus; ultimo sat magno, carinato (carina acuta pallidior et lavigata), supra declivi-subconvexiusculo et ad insertionem breviter descendente, subtus ad initium recte declivi ac deinde paulation ad aperturam convexiore el circa umbilicum obscure subangulato; apertura obliqua, vix lunata, superne recte declivi, inferne exacte convexo-rotundata; peristomate recto, acuto, intus labiato, ad columellam subdilatato; alt. 5 , diam. 9 millim.

Collines d'El-Kef (Let.).

Le type a été trouvé à Tipaza (Pechaud), dans la province d'Alger.

\section{\$ 71 .}

II. Uineiformis Letourneux et Bourguignal, sp. nov.

Testa profunde umbilicata (umbilicus sat angustus, nihilominus pervius), depressa, angulata (angulus ad aperturam leviter obsoletus), supra

(1) Bourg. 1883. (Helix finitima Morelet, in Journ. Conch. $187^{6}$, p. 374, et Moll. Maroc, p. 35, pl. I, fig. 2, 1880. - Non Helix finitima de Ferussac 1821, qui est cette espèce que le mème antenr a dibaptisée en 1854 pour lui imposer le nom d'H. Calpeana.) 
tholiformiter convexiuscula, subtus convexiore, parum nitida, subtranslucida, fragili, regulariter striala (striæ in supremis sat tenues, in ultimo validiores), albescente cum zonulis 6 fuscis quarum superior latior ac flammulis albis interrupta; spira convexiuscula, tholiformi (apex corneus, nitidus, lævissimus); anfractibus 6 carinatis (carina suturam sequens), lente crescentibus, superne convexiusculis; ultimo vix majore, angulato, supra parum convexo et ad insertionem recto, subtus bene convexo; apertura obliqua, sat lunata, externe subangulata, superne leviter arcuata, ad basin exacte rotundata; peristomate recto acuto, intus vix subincrassatulo; alt. 5 , diam. 9 millim.

Sous les pierres, au Kef el-Djerdja (Let.).

Cette espèce se distingue de l'H. Tineana (Benoît, Ill. Test. Sicil. [IV, 1862], p. 185 , pl. IV, f. 24 ), par sa taille moindre, par son test fragile, subtransparent, plus délicatement strié et moins fortement caréné, par son ombilic moins ouvert, par son péristome simple, tranchant et sans ce gros bourrelet orangé caractéristique de l'ouverture de l'espèce sicilienne.

\section{\$ 72.}

I. Tristami L. Pfeiffer, Desc. land shells, in Proc. zool. Soc. Lond. 186o, p. 136, et Bourguignat, Mal. Tunis, p. 23 , pl. I, f. 28-30, 1868.

On ne connait pas l'habitat exact de cette Hélice; on présume qu'elle vit dans le Djebel Zaghouan. En Algérie, cette espèce a été constatée sur les sommités de Lalla-Khedidja, dans la chaîne du Djurjura.

\section{Tissotiana Bourguignat, sp. nov.}

Testa profunde umbilicata (umbilicus pervius, nihilominus mediocriler apertus), depressa, acute carinata (carina alba, fimbriata), superne subtusque relative parum convexa, non nitente, opacula, subcretacea, uniformiter cinerascente, crebre valideque striata; spira convexiuscula (apex nitidus, corneus, lævigatus); anfractibus 6 arcte spiratis, leviter subscalariformibus, carinatis (carina in prioribus suturam sequens, in ullimis supereminens), superne vix convexiusculis; ultimo vix majore, relative exiguo, acute carinato, supra convexiusculo ac ad insertionem regulariter valideque descendente, subtus convexo, ad initium subtectiformi, circa umbilicum turgidulo; apertura vix obliqua, non lunata, transverse oblonga, externe angulata; peristomate fere continuo, recto, acuto, inferne leviter subpatulo; alt. 5, diam. 12 millim.

Sous les pierres, entre les ruines d'Oudena et les premiers contreforts du Djebel Reças (Bourg. 1867).

Cette Hélice, si remarquable par la forme de son ouverture et par son péristome presque continu, est dédiée à M. Ch. Tissot, ancien consul à Tunis. 
H. Zeugitana Letoumenx et Bourguignat, sp. nov.

Testa aperte lateque umbilicata (umbilicus amplus, exacte pervius), acute carinala (carina stricla, sæpe fimbriala), supra omnino plana, subtus plus minusve tectiformi-convexa et ad umbilicum angulala, non nitente, subopacula, uniformiter albescente aut cinerea, valide striala (striæ regulares, crebræ, super carinam validiores); spira complanata (apex minutus, corneus, lavigatus); anfractibus 6 arcte crescentibus, carinatis, supra planulatis ac circa carinam aliquando prominentem subconcaviusculis, sulura lineari separatis; ultimo vix majore, valide carinato, supra planulato, ad carinam sulculo concaviusculo cinclo ac ad insertionem regulariter lenteque descendente, subtus ad initium e carina usque ad umbilicum lecliformi et prope aperturam convexiusculo, landem circa umbilicum turgido ac angulato; apertura leviter obliqua, irregulariter subquidrala; peristomate recto et acuto; alt. 3, diam. 12 millim.

Sous les détritus, dans le Djebel Zaghouan (Lel.).

Dans le Djebel Reças, on rencontre une variété à ombilic un peu moins ouvert.

$$
\text { \$ } 73 .
$$

Les sept Ilélices qui suivent fout partie d'un groupe fort nombreux, le gronpe de l'I. amandu, dont les différentes espèces sont réparties dans presque toutes les régions méditerranéennes. Parni elles, nous citerons : les II. Hyperconica, tellica, Brondeli, ablennia, copora, hodnica, Henoniana (Bourg.) d'Algéric; les 11 . tervira et amandella (Let.) également d'Algérie; Ies II. dormiens (Benoit) et pleurischura (Bourg.) de Sicile, dont la première a été retronvéc dans nos possessions du nord de l'Afrique; les II. Syrensis (Pfeiffer), syrosina et syronesiaca (Bourg.) des îles de l'Archipel el de Syrie; l'H. monservalica (Bourg. ${ }^{(1)}$ d'Espagne; l'H. Ledereri (I'friffer) de Syric el d'Égypte, elc.

I. I rianensis Bourguignat, Malac. Tunis, p. 21, pl. I, f. 22-35, 1868.

Très abondante dans les jardiıs de l'Ariana, près de Tunis (Bourg. 1867). Le $\mathrm{D}^{r}$ Gestro $(1876)$ a également recueilli cette espèce aux environs d'Uticque, et le Dr P. Marès an pied du Djebel lieças.

I. Calistoderma Letournenx et Bourguignat, sp. nov.

Testa profunde unbilicata (umbilicus perangustus, nihilominus pervius), angulata, subconico-depressa, non nitente, subopacula cum milculis subtranslucidis, eleganter costulata (costx validæ, regulares, sat inter se distantes, producte, in ingulo validiores et circa umbilicum evanescentes), albescente cum zonulis 3 (una superior, alteræ inferiores) interruptis, corneis quasi oleatis; spira subconoidea, parum producta

(1) Helix Monserratensis d'Hidalgo. 
(apex minutus, corneus, nitidus ac lævigatus); anfractibus $5 \frac{1}{2}$ regulariter lenteque crescentibus, superne convexis, sutura impressa separatis; ultimo vix majore, angulato (angulus fimbriatus, ad aperturam obsoletus), supra convexo, ad insertionem vix descendente, subtus subtectiformi-convexiusculo et circa umbilicum turgidulo; apertura parum obliqua, vix lunata, subovata; peristomate recto, acuto, intus valide albolabiato; alt. 5 , diam. 9 millim.

Sous les pierres, à Guelaat-es-Snan (Let.).

\section{H. Enfidana Letourneux et Bourguignat, sp. nov.}

Testa angustissime ac perprofunde umbilicata (umbilicus punctiformis, nihilominus pervius), acute angulata, depressa, supra tectiformi-conoidea, subtus convexiuscula, sat nitida, opacula, valide striata (striæ regulares, super angulum validiores), albescente, supra maculis atris ornata et subtus zonulis ( 2 vel 3 ) atris, sæpe conjunctis et semper interruptis, cincta; spira conoidea, tectiformi (apex minutus, corneus, nitidus ac lævis); anfractibus 6 regulariter crescentibus, tectiformibus, fere planulatis, sutura lineari separatis; ultimo relative majore, angulato, supra convexiore; apertura parum obliqua, sat lunata, obscure subquadrangulari, externe angulata; peristomate acuto, intus leviter incrassato; • alt. $4 \frac{1}{2}$, diam. 7 millim.

Dans les lieux arides, au pied des buissons, à l'Enfida (Let.).

Au Djebel Zaghouan, on rencontre une variété d'une taille légèrement plus forte (haut. 5 , diam. $8 \frac{1}{2}{ }^{\mathrm{mm}}$ ) avec une teinte fauve-cornée uniforme.

\section{H. Morini Bourguignnat, sp. nov.}

Tesla profunde umbilicata (umbilicus mediocriter apertus, pervius), depressa, angulata, supra parum convexa, subtus convexiore, non nitente, opaca, subcretacea, sat sordide striato-costulata (costæ super angulum validiores), unifurmiter albido-lutescente; spira depresso-tectiformi, parum convexa (apex exiguus, corneus, nitidissimus ac lævis); anfractibus $5 \frac{1}{2}$ angulatis, lente usque ad ultimum, dein rapidius crescentibus, supra vix convexiusculis, sutura impressula separatis; ultimo majore, angulato, supra convexiusculo-subtectiformi, ad insertionem recto, sulstus convexo, ad umbilicum leviter turgidulo; apertura parum obliqua, mediocriter lunata, subrotundata, superne rectiuscula, externe angulata, ad basin sphærica, intus splendide candida; peristomate recto, acuto, intus profunde albolabiato; margine columellari sat robusto, patulescente; alt. 5, diam. 9 millim.

Cette Hélice, dédiée au sous-lieutenant Eugène Morin, vit au Bardo, sur le talus des fossés. 
H. ehthamatolena Bourguignat, Spec. noviss. Moll. $n^{\circ} 51,1876$.

Cette espèce algérienne, découverte aux environs d'Oran et sur le territoire des Ouled Djebara, a été constatée, en Tunisie, dans le Djebel Reças el dans le Djebel Bou-Kourneïn (Let.).

II. amanda Rossmässler, Iconogr. VII et VIII, 1838, f. 449 et Bourguignat, Malac. Alg. I, 1864 , p. 296 , pl. XXXI, f. $34-33$.

Cette Hélice sicilienne, constatée en Algérie, aux environs de Mostagihanem, notamment à Pelissier, ainsi qu'aux alentours d'Alger (Muslapla, Guyotville) ef de Laghouat, a été recueillie en Tunisie, près de Porto-Farina (Let.), où elle paraîl assez rare.

M. le prolesseur A. Issel (Groc. del Violante, Moll. in Ann. mus. civ. Genova, 1880, p. $27^{0}$ ) a siğnalé dans l'ile de la Galite et sur les îlots Galitone et Gallina une variété insularis qui paraît être assez différente du type.

H. tunetana $L$. Ifeiffer, in Zeitschr. f. Malak. p. $7^{0}, 1850$, et Monogr. Hel. viv. III, 1853 , p. $160 \mathrm{ct}$ in Chennitz ( $3^{\circ}$ édit.), Helix, $n^{\circ} 864$, tab. CXXXIV, f. $3-4$ et Bourguignal, Mal. Tumis, p. 22, pl. I, 26-27, 1868.

On ne connait pas l'habitat de cette Hélice. L. Pfeiffer l'indique des environs de Tunis, où elle n’a pas été retrouvée.

\section{S 74.}

1. Lyperplataea Servain, Moll. Esp. p. 11 h, 1880.

Celle singulière espèce espagnole, dı groupe de l'II. catocyphia (x), a été r'ecupillie dans le sud de la Tunisie, aux environs de Fralis et de Zarzis (Let.), où elle vit dans les endroits arides et brûlés par les rayons du soleil.

\section{$\$ 75$.}

11. Conicula Letourneux el Bourguignat, sp. nov.

Testa minuta, profunde el peranguste perforata, angulala, conoidea, parum nitida, opaca, crelacea nihilominus vix crassa, uniformiter ablbescente, bene striata (stria in angulo subtuberculosæ); spira conoidea, sat producta, ad summum obtusa (apex relative amplus, nitidus, lavis ac corneus); anfractibus $5 \frac{1}{2}$ angulalis, perlente crescentibus, superne convexis, sutura profunda separatis; ultimo vix majore, angulato (angulus ad aperturam obsoletus), supra sublusque convexo, ad insertionem regulariter lenteque descendente; apertura vix obliqua, sal lunata, transverse ovato-hemisphrrica; peristomate recto, acuto, intus incrassatulo; all. 4, diam. 5 millim.

Pelouses arides près de Zarzis (Lel.).

Variété un peu plus forte et moins élancée aux environs de Fratis (Duv.).

12) Bourg. Malac. Chàt. d'If, p. 13 , pl. I, f. $1-3,1860$. 


\section{\$ 76 .}

Le groupe de l'Helix pyramidata, qui n'a jamais été le sujet d'une étude sérieuse, comprend, à notre connaissance, plus d'une trentaine d'espèces, sur lesquelles seize ont été constatées en Tunisie.

Les Hélices de ce groüpe peuvent se répartir en quatre séries : $1^{\circ}$ en espèces à spire très turriculée; $2^{\circ}$ en espèces à ouverture ronde ou subarrondie; $3^{\circ}$ en espèces bien ombiliquées; enfin, $4^{\circ}$ en vraies pyramidelles, qui elles-mêmes se subdivisent en espèces ả coquille presque lisse ou à test costulé.

Les Hélices tunisiennes de la première série sont les $H$. newskopsis, eupyramis et Tisemsinica; a celte série se rattachent les H. Idalice (Bourğ.), Liebetruti (Albers) de Chypre, l'H. Newska (Dohrn) des îles Baléares et les H. pyramis (Philippi) et Licodiensis (Cafici) de Sicile.

Celles de la seconde série sont les $H$. Zitoumica, Madana, Capuana et Tarentina, auxquelles il faut joindre les $H$. Setiliana (Bourg.), Zahresina (Bourg.) el Ouesserana (Pechaud) d'Algérie, ainsi que l'H. euopsis (Bourg.) d'Italie.

Celles de la troisième sont les $H$. galactina, Dyracchiensis, Veneriana, mumidica et anombra, auxquelles il convient d'ajouter l'II. subnumidica (Bourg.) de Sicile, l'H. Sittianomum (Bourg.) d'Algérie et l'H. Cornaroi (Berthier) de Dalmatie, d'Albanie et d'Algérie.

Enfin, celles de la quatrième série sont : $1^{\circ}$ chez les lisses ou peu striées, l' $H$. pyramidata, de laquelle tendent à se rapprocher les $H$. Lycabetica (Let.), tremesia et Vardeorum (Bourg.) d'Anatolie, de Grèce, de Dalmatie, d'Italie et même des côtes de la Provence; $2^{\circ}$ chez les costulées, les $H$. spaella, spaellina et mactanica, auxquelles il est juste d'adjoindre les $H$. apiculus (Rossm.) et apiculiformis (Ancey) de l'île Lampedouse.

Comme on le voit, le groupe de l'H. pyramidata atteint son maximum de développement en Tunisie. C'est, en effet, dans ce pays que l'on rencontre les plus beaux échantillons et la plus grande diversité de formes.

\section{II. newskopsis Letourneux et Bourguignat, sp. nov.}

Testa punctiformi-perforata, alte pyramidata, carinata cum simulatione secundæ carinæ superæ in ultimo præsertim perspicuæ, non nitente, opaca, subcretacea, uniformiter candida, subtilissime striata (striæ sæpo in carina validiores ac quasi subtuberculosæ); spira alta, subtectiformiconica, ad summum obtusiuscula (apex minutus, corneus ac lævis); anfractibus 8-9 arcte spiratis (priores tectiformes ac planulati, inferiores convexiusculi), sutura inter supremos lineari, inter ultimos sat profunda separatis; ultimo vix majore, ad peripheriam carinato ac insuper obscure subangulato (carina et angulus sulculo parum distincto separati), superne convexiusculo, ad insertionem recto, subtus parum convexo, quasi compresso; apertura obliqua, lunata, transverse semioblonga, externe angulata; peristomate recto, acuto, inferne leviter patulo, intus valide albolabiato; alt. et diam. æque $8^{\mathrm{mm}} \frac{1}{2}$. 
Dans le sable, près Hammam-el-Lif et dans les dunes, entre Nebeul et Mlenzel Temim (Let.).

H. eupyramis Letourneux et Bourguignat, sp. nov.

Testa angustissime perforata, alte pyramidata, nunquam carinata, sat nitente, opaca, subcretacea, uniformiter candida, argutissime striatula quasi sublævigata; spira alta, conoidea, ad summum obtusa (apex corneus ac lævis); anfractibus 7 arcte spiratis, convexiusculis, sulura impressa, ad ultimum profunda, separatis; ultimo mediocri, compressorotundato, supra convexo, ad insertionem recto, subtus compresso; apertura obliqua, sat lunata, transverse in directionem leviter declivem semioblonga; peristomate recto, acuto, inferne subpatulescente, intus valide albo-labiato; alt. et diam. æque 10 millim.

Le type de l'H. eupyramis se trouve à Aïn-Tonkria, hameau à 32 kilomìtres au sud de Teniet-el-Haad, dans nos possessions algériennes, où cette Hélice a encore été constatée à Mostaghanem, ainsi que dans la dépression sablonneuse du Zahrè̀s, au sud de Guelt-el-Settel, et entre Aïn-Ouessera et Bon-Ghezoul, au sud de Boghar (l).

Eu Tunisie, l'H. enpyramis a été recueillie aux environs de Tunis, sur les ruines d'Utique (Bourg. 1867), à Hammam-el-Lif, à Menzel-bou-Zelfa, à Foum Bouibet (Let.).

Dans les dunes entre Nebeul et Menzel-Temim, on rencontre une variété obtusalis et au Kef une forme minor de cette espèce.

Les différences entre cette Hélice non carénée et la précédente sont si prononcées que nous croyons superflu de les énoncer.

\section{Tisemsiniea Bourguignat, sp. nov. 1880.}

Testa vix perforata (perforatio punctulo minutissimo similis), alta, pyramidato-globosa, valde nitida, opaca, persubtiliter striatula vel polius lavigata, candida cum maculis luteolis perdilutis, superne regulariter distantibus, subtus irregulariter sparsis, et zonula atra albo-interrupta cleganter cincta; spira producta, inflata, conoideo-globosa, ad summum relative peroblusa (apex exiguus, castaneus, perlævis); anfractibus 6-7, usque ad ultimum arcte spiratis, dein leviter rapidius crescentibus, convexis, sulura profunda separatis; ultimo leviter majore, convexo, ad aperturam rotundato, superne ad insertionem leviter subdescendente; apertura parum obliqua, lunata, liemisphærica; peristomate recto, acuto, intus albo-albiato; alt. 7 , diam. $7^{\mathrm{mm}} \frac{1}{2}$.

C'est aux environs d'Aüı-Tisemsin, entre Aïn-Toukria et le plateau du Sersou

(i) L'H. eupyramis vit aussi, à notre connaissance, aux environs d'Athènes (Grèce), de Ragnuse (Dalmatie) et mème dans l'Esterel, entre Fréjus et Cannes et à Saiut-Nazaire, près Toulon (Var). 
(province d'Alger), que se trouve le type. En Tunisie, on n'a rencontré jusquà présent qu'une forme un tant soit peu plus petite aux alentour's de Zarzis (Duveyrier).

Cette Hélice peut servir de trait de transition entre les espèces de la première série et celles de la seconde.

Par sa forme élancée-conoïde, elle appartient, en effet, à la série des turriculées, tandis que, par sa bouche hémisphérique, elle se rapproche de celles à ouverture arrondie, que nous allons faire connaître.

\section{H. Ritoumica Letourneux et Bourguignat, sp. nov.}

Testa puncliformi-perforata, conoidali, nitidissima, opaca, bene striata, subfulvo-albescente cum zonulis 2 angustis (una ad peripheriam, altera circa perforationem) aut cum zonula atra supera (unica rel bipartita), sæpe interrupta et aliquando prope suturam cum flammulis castaneis inter se æque distantibus; spira conoidali, sal producta, nihilominus leviter depressa, ad summum acutiuscula (apex prominulus, caslaneus, lævissimus ac minutissimus); anfractibus 7 regulariter ac sat lente crescentibus, convexis, sutura relative profunda separalis; ultimo sat amplo, ad initium subcompressiusculo, ad aperturam subrotundato, superne ad insertionem recto; apertura vix obliqua, lunata, hemisphærica ; peristomate recto, acuto, ad basin subpatulo, inlus profunde incrassato; alt. 9, diam. 12 millim.

Le long de l'Oued Zitoum (Let.).

Variété minor dans les plaines, au-dessous d'El-Kef (Let.).

H. Madana Letourneux et Bourguignat, sp. nov.

Testa puncliformi-perforata, depressa, ventroso-subconoidali, parum nitente, cretacea, opaca, argute striata, uniformiter albescente, rare cum zonula castanea ad peripheriam; spira mediocriter producta, obluse subconoidali (apex corneus, lævis); anfractibus 6-7 regulariter lenteque crescentibus, convexis, sutura profunda separalis; ultimo sat majore ac ventroso, rotundato, ad insertionem recto; apertura vix obliqua, lunata, hemisphærica; peristomate recto, acuto, inferne patulescente, intus incrassato; alt. 7 , diam. 11 millim.

Plaines au-dessous d'El-Kef, Oued Zitoum et Foum Bouibet (Let.).

En Algérie, l'H. Madana vit aux environs de Tebessa et entre Bou-Ghezoul et Boghari.

Nous la connaissons également de la Sainte-Baume, dans le Var.

I1. Capuana Bourguignat, in coll. 1875 .

Testa profunde ac relative sat aperte umbilicata, depressa, ventrosa, superne subconoidali-convexa, nilente, cretacea, opaca, argute striatula, albescente, rare zonulis castaneis angustisque (una ad peripheriam, altera 
inferior) circumcincta; spira depressa, convexa, perobtusa (apex minutus, corneus ac lævis); anfractibus 6-7, regulariter ac subvelociter crescentibus, convexis, ad suturam perprofundam subturgidulis; ultimo majore, rotundato, ad insertionem leviter descendente; apertura parum obliqua ac lunata, hemisphærica; peristomate recto, aculo, parum incrassato; margine columellari robusto, crassulo ac patulo; alt. 7 , diam. 11 millim.

Le type se trouve dans le parc royal de Capone (Italic), où vraisemblablemeni il a été importé d'Afrique.

Eil Tunisie, on rencontre l'II. Capuana aux environs de Tunis (Bourg. 1867) et "l'El-Kef (Let.).

Nous comnaissons encore celte espèce d'Ainl-Ouessera, près de Bou-Ghezoul, dalls la province d'Alger', et de Ramleh, près d'Alexandrie, en Égypte.

II. Tarrentina $L$. Pfeiffer, Monogr. Hel. viv. 1, 1848, p. 442 (Helix pyramidata, var. Philippi, Moll. Sicilix II, 1844, p. 110).

Cette llélice italieune et sicilienne a été relrouvéc aux environs d'El-Kef (Let.)

Ou a également constaté, en Algérie, la présence de l'H. Tarentina, daus le 1)jebel Sahıri, près Djelfa.

\section{H. galactina Letourneux et Bourguignat, sp. nov.}

Testa profunde angusteque umbilicata, depressa, angulata (angulus fimbriatus), supra subtusque fere equaliter convexa, subnitente, cretacea, opaca, candida, valide striata (strix in angulo validiores); spira subconoidali-depressa, mediocriter producto-convexa, ad summum acutiuscula (apex aler, nitidissimus ac perlævis); anfractibus 6 regulariter ac sat lente crescentilous, convexis, sutura profunda separatis; ultimo vix majore, compresso, angulato (angulus id aperturam leviter obsoletus), supra subtusque convexo, ad insertionen recto; apertura vix obliqua, lunata, transverse seni-oblonga, intus lutea; peristomate recto, acuto, intus albo-incrassato; alt. 5 1/2, diam. $10 \mathrm{millim}$.

Environs de Zarzis (Let.).

Forme d'une taille un peu moindre el entourée d'une bande marron, aux alentours de Ras El-Djerf, dans l'ile de Djerba (Lel.).

H. Dyrrachiensis Bourguignat, sp. nov. 1880 .

Testit profunde umbilicata (umbilicus pervius, sal apertus), depressia, angulala, supra tectiformi-convexa, subnitida, sat fragili, subopaculi, striatula, uniformiter albescente aut alba cum zounlis 2 fuscis filiformibus quarum una ad peripherian late interrupta et altera inferior continua ac diluta; spira parum producta, mediocriter convexa, obtusa (apex corncus ac lævis); anfractibus 6 regulariter ac sat lente crescen- 
tibus, convexis, sutura impressa separatis; ultimo mediocri, compressorotundato, angulato, ad insertionem recto; apertura vix obliqua, parum lunata, transverse oblonga; peristomate recto, acuto, intus vix incrassatulo; alt. 4, diam. 7 millim.

Le type a été recueilli en Albanie, aux environs de Durazzo.

En Tunisie, cette espèce se trouve représentée par une forme un peu plus grande, à test un peu moins délicat, trouvée à Foum Bouibet (Let).

\section{H. Veneriana Letourneux et Bourguignat, sp. nov.}

Testa profunde lateque umbilicata (umbilicus apertus, pervius, in ultimo dilatatus), depressa, angulata, supra subtusque fere æque convexa, subnitente, opaca, cretacea, bene striata, uniformiter albescente aut lutescente, rare zonula fusca cincta; spira mediocriter convexa, perobtusa (apex minutus, corneus ac lævis); anfractibus 6 convexis, regulariter crescentibus, sutura sat profunda separatis; ultimo sat majore, compresso, angulato (angulus ad aperturam obsoletus), convexo, ad insertionem reclo; apertura vix obliqua, lunata, transverse subovata; peristomate recto, acuto, intus incrassato; alt. 6 , diam. 10 millim.

Plaine d'El-Kef, et à Foum Bouibet, où l'on trouve également, mais assez l'alement, une variété sans angulosité (Let.).

En Algérie, cette Hélice a été constatée aux environs de Tebessa.

If. numidiea Moquin-Tandon in L. Pfeiffer, Helix (a édit. Chemnitz), $\mathrm{n}^{\circ} 712$, pl.CXIX.

f. 3-1 et Monogr. Hel. viv. I, p. 444, 1848.

Le type de cette espèce se rencontre aux environs de Constantiue, ainsi quä Setif ${ }^{(1)}$.

En Tunisie, l'H. numidica a été constatée près de Foum Bouibet (Let.) ${ }^{(2)}$.

II. anombra Bourguignat, sp. nov.

Testa profunde pervie aperteque umbilicata, conoidali, acute carinata (carina stricta, prominens, nitidissima), nitente, subopacula, persubliliter striatula, uniformiter fulvo-albescente, raro fusco-zonata; spira sat alta, obtuse conica (apex fere ater, perlævis ac pernitidus); anfraclibus 6-7 lente crescentibus, supra convexis, ad peripheriam acute carinatis (carina stricta, suturam linearem sequens ac super illam projecta); ultimo vix majore, carinato, ad initium subcompresso, ad aperturam regulariter convexiore, ad insertionem recto; apertura vix obliqua, lunata,

(1) Cette Hélice a été également signalée au Chàteau d'If, près Marseille (Bourg. Mal. Chât. d'If, p. 16, pl. I, f. 4-6, 1860).

(2) Cette Hélice se trouve encore assez abondamnent dans des sacs de céréales provenant du centre du pays. 
transverse semioblonga, intus luteola; perislomale recto, acuto, inferne patulescente, inlus profunde incrassato; alt. $61 / 2$, diam. 10 millim.

Cette jolie Hélice, si remarquable par sa carène, a été découverte cu Algérie, près de Mesran, puis au col de Guelt-es-Settel, entre Djelfa et Boghar, enfin à Tebessa.

En Tunisie, elle a été constatée dans la plaine d'El-Kef, ainsi qu'à Khanguet Slougui (Let.).

H. pyramidata Draparnaud, Hist. Moll. Fr. p. 8o, pl. V, f. 5-6, 1805 .

Gette espèce est une des Hélices les plus comnumes de la Tunisie; on la rencontre partout, du nord au midi, de l'est à l'ouest; aussi croyons-nous superflu de citer les nombrenses stations où elle a été recneillie.

I. spaella Letourneux et Bourguighat, sp. nov.

Testa profunde angusteque perforata, obluse conica, angulata, subnilida, cretacea, opaca, uniformiter candida et rarius fusco-zonata aut flammulata, valide costulata (cosł crassæ, productæ ac regulares); spira sat producta, obtuse pyramidata (apex minutissimus, corneus ac lavis); anfractibus 7 convexis, regulariter lenteque crescentibus, sulura profunda separatis; ultimo sat majore, angulato (angulus plus minusve obsoletus), supra convexo, ad insertionem recto, subtus ad initium colirpressiusculo, ad aperturan convexiore; apertura obliqua, lunata, sat hemisphærica; peristomate recto, acuto, intus incrassato; alt. 8, diam. 11 millim.

Amphithéâtre d'El-Djell, où se trouve le type (Let.).

Environs de Tunis (Bourg. 1867), Djebel Mezemzen au Cap Bon, Mehedia, Henchir Goutin près Metaneur, Bir el-Ahmar, montagne de Matmata, enfin, Houmt-Souk dans l'île de Djerloa (Let.).

II. spaellina Letourneux el Bourguignat, sp. nov.

Testa profunde perforata, pyramidali, angulata, subnitida, opaca, plus minusve cretacea, uniformiter candida, raro fusco-zonata aut flammulata, valide costata (costæ super angulum aliquando tuberculos $x$ ); spira plus minusve producta, pyramidali (apex castaneus, lee is, nitidus); anfractibus 6 convexis (priores usque ad ultimum graciliores), lente usque ad ultimum, dein rapidius, crescentibus, sutura profunda separatis; ultimo majore, relative sat amplo, angulato, compresso, ad insertionem reclo; apertura obliqua bene lunata, transverse semioblonga; peristomate reclo, acuto, intus valide incrassato; alt. 5 , diam. 8 millim.

Le type provient de Meliedia.

Cette espèce, qui semble plus méridionale que la précédente, a été trourée à Gabès, Fralis, Zarzis, Ras El-Djerf vis-à-vis de Djerba; enfin, dans le lit de l'Oued 
entre Djerf Ouman el-Azir et Lemdou, et sur les bords de l'Oued Zegzaou, près d'Aram (Let.).

L'H. spacllina se distingue de lH. spaella par sa taille moindre, par son ouverture plus échancrée, non hémisphérique, mais semi-oblongue dans le sens transversal, par sa croissance spirale lente jusqu'au dernier tour, puis plus rapide; enfin, surtout, par sa spire composée de tours grêles, paraissant comme juxtaposée sur le dernier, par suite du grand développement relatif de celui-ci. Chez l'H. spaella, la croissance spirale est fort régulière, et il n'y a aucune disparité sensible de proportion entre les tours.

II. Mactaniea Bourguignat, sp. nov.

Testa punctiformi-perforala, carinata, tectiformi, plus minusve pyramidata, subtus valde compressa ac parum convexa, nitidula, opacula, costulata, albescente ac fere semper super spiram (rarissime ad inferiorem partem ultimi) zonulis fuscis continuis aut plus minusve interruplis et flammulis regulariter sparsis ornata; spira plus minusve producta, tectiformi-conica, ad summum acutiuscula (apex minulissimus corneus ac lævis); anfractibus 6-7 convexis, regulariter crescentibus, sutura impressa separatis; ultimo parum majore, valide carinato, supra convexo, ad insertionem breviter descendente, subtus compresso parum convexo; apertura subobliqua, lunata, transverse semioblonga; peristomate recto, acuto, intus albo-incrassatulo; alt. 6 , diam. 8 millim.

Cette belle espèce a été découverte d'abord à Oran, puis aux environs d'Alger.

En Tunisie, où elle paraît abondante, elle a été constatée au Djebel Mezemzem (Cap Bon), à Kelibia, à l'amplithéâtre d'El-Djem, à Henchir Goutin près de Metameur, à Bir el-Ahmar, sur les bords de l'Oned el-Fetour près de Matmala, à Fratis, à Zarzis, au Ras El-Djerf vis-à-vis de Djerba; enfin dans les détritus de l'Oued Sidi-Aïch, de l'Oued Zegzaou près d'Aram, ainsi que dans le lit de l'Oued entre Djerf Ouman el-Azir et Lemdou (Let.).

\section{\$ 77.}

L'Hélice que nous allons signaler appartient à un petit groupe d'espèces carénées à spire conique-tectiforme, dont les plus importantes sont l'H. Cumice (Calcara) de Sicile, l'H. Lampedusce (Bourg.) de l'île Lampedouse, l'H. Nivaricnsis (Shuttleworth) des îles Canaries, qui a été retrouvée acclimatée au pied des oliviers, entre Grasse et l'Esterel, dans les Alpes-Maritimes.

\section{Kelibiana Letourneux et Bourguignat, sp. nov.}

Testa punctiformi-subperforata (perforatio superficialis, punctulo minutissimo similis), tectiformi-conica, angulata, fragili, subpellucida, nilidula, subtiliter striatula, pallide lutescente cum zonulis 3 fuscis late interruptis quarum una ad peripheriam super angulum, alteræ inferiores; spira producta, conica, ad summum acutiuscula (apex corneus 
lævis ac minutissimus); anfractibus 6 convexiusculis, lente crescentibus, sulura impressa separalis; ultimo vix majore, angulato, supra subconvexo; apertura leviter obliqua, valde lunata, transverse suboblonga, externe angulala; peristomate recto, acuto, vix labiato; alt. 7 , diam. 8 millim.

Environs de Kelibia (Let.).

\section{\$ 78}

Trois espèces seulement du groupe de l'II. terrestris ont été rencontrées en Tunisie.

I. scitnla Jan et Cristoforis, Cat. rerum natural., Mantissa, p. 2, 1833.

El-Haouiria, dunes entre Nebeul et Menzel-Temim (Let.).

II. terrestris Chemnitz, Conch. cab. IX ( $a^{\circ}$ partie) 1786 , p. 47 , pl. CXXII, f. 1045 (Helix elegans de Gmelin, Draparnaud, etc.).

Espèce abondante aux environs de Tunis, sur les ruines d'Utique, de Carthage et d'Oudena (Bourg. 1867); alentours de Bizerte (Bellucci, 1875), de Porto-Farina, d'llammam-el-Lif (Let.).

Au Bardo, l'on trouve de magnifiques échantillons flammulés de très grande taille (Bourg.).

m. trochlea L. Pfeiffer, Symb. ad Hist. Hel. viv. III, p. 69, 18 16.

Environs de Kamart, ruines de Carthage au pied des oliviers; versant est dı Djebel Ahmar (Bourg. 1867).

L'II. trochlea a été constatée en Algérie, aux environs de Bône, de Bongie el d'Alger.

\section{S 79.}

Les espèces du groupe de l'II. acuta sont les dernières que nous ayons ì signaler de la Tunisie.

II. trochoides Poiret, Voy. Barbarie II, ${ }_{7} 79$, p. 29 .

Cetı Hélice est une espèce essentiellement africaine que nous ne connaissons que de la Tunisie et de l'Algéric.

l:Helix conica(1), quo l'on a jusqu'à présent confondue avec celle-ci, doil en être séparée, ainsi que l’a fait observer fort judicieusement notre ami de SaintSimon dans son Étude des IIelix du groupe de l'II. elegans ${ }^{(2)}$.

L'H. conica ${ }^{(3)}$, en effet, est plus turriculée, plus carénée, et la partic supérieure

(1) Draparnaud, Tabl. Moll. p. 69, 1801 et Hist. Moll. Fr. p. 79 , pl. V, f. 3-5, 1805.

(2) Toulouse, 188 s.

(3) Le type de l'H. conica se trouve sur le littoral du département de l'Hérault. C'est une espèce rópandur dans presque toutes les contrées côtières d'Espagne, de France, d'Italie et mème de Dalmatie. 
du dernier tour, au-dessus de la carène, est toujours gonflée, comme subanguleuse, au lieu d'être simplement convexe comme celle de l'H. trochoides.

La vraie $H$. trochoides de Poiret, qui est très abondante aux environs de Bône et de la Calle, ainsi que sur différents antres points de l'Algérie, a été constatée en Tunisie, aux alentours de Tunis, Carthage, Utique, Oudena (Bourg. 1867), au cap Bon (Bell. 1875), à la Galite (Gestro), ainsi qu'à l'île de Tabarque (Let.).

Cette espèce a encore été recueillie à Hammam-el-Lif, à Menzel-bon-Zelfa, à ElHaouiria, à Kelibia et dans les sables et les dunes de Nebeul, de Menzel-Temin, de Zouzag, de Counara et de Sidi-Daoud (Let.).

H. conoidea Draparnaud, Tabl. Moll. p. 68,1801 et Hist. Moll. p. 78 , pl. V, f. 7-8, 1805 .

De Sousse à El-Buita (Gestro 1876 ); sables à Hammam-el-Lif, à Mehedia, à Hammamet (Let.); au Djebel Zaghouan (Doûm.-Adans.).

On rencontre assez rarement sur les ruines de Carthage et à Hammamet une forme singulière qui mériterait d'être élevée au rang spécifique si elle était plus abondante, l'Helix Kerimi (Bourg.), dédiée au Persan Kerim, un des nombreux explorateurs de la Tunisie.

Cette forme diffère de la véritable $H$. conoidea par son test plus large à la base, par conséquent moins turriculé, par une perforation moins ouverte et par une ouverture plus oblique, légèrement plus ovalaire dans un sens incliné de gauche à droite.

\section{H. Bellneeiana Bourguignat, 1880 .}

Testa punctiformi-perforata, ad basin ampla, late conoidali, angulata (angulus fimbriatus), sat fragili, subpellucida, parum nitente, persubtiliter striatula, uniformiter albescente; spira producto-pyramidali ac obtusa (apex minutissimus); anfractibus $7-8$ convexiusculis, arcte spiratis, sutura bene impressa separatis; ultimo vix majore, angulato (angulus ad initium fimbriatus, ad aperturam obsoletus), supra convexiusculo, ad insertionem recto, subtus compresso, parum convexo; apertura leviter obliqua, perlunata, semiovata; peristonate reclo, acuto, fragiti, non incrassato; margine columellari tenui superne in triangulam formam reflexo; alt. et diam. æque $81 / 2$ millim.

Gette belle Hélice, bien que nous l'ayons placée entre les $H$. conoidea et barbara, ne peut être assimilée à ces deux espèces, dont elle se distingue notamment par sa taille aussi large que haute, non moins que par ses tours très serrés, dont le dernier est fortement comprimé en dessous.

L'H. Bellucciana, dédiée au savant professeur italien Bellucci, paraît rare; elle a été trouvée sur les bords du lac El-Bahira (Bertbier).

H. barbara Linnaeus, Syst. nat. (ed. X, 1758), p. 773 , et Bourguignat, Mal. Alg. I, 
1864 , p. 286 , pl. XXXII, f. 36-41 (Bulimus ventricosus, Draparnaud, 1801 el 1805 ;

Helix ventricosa, Ferussac, 1823; Helix bulimoides, Moquin-Tandon, 1855).

Sur les herbes, aux environs de Tumis, à la Mahamedia, à Oudena (Bourg. ${ }_{186}$ ); bords de la Medjerda (Gestro, 1876 ).

H. aeuta Müller, Verm. Hist.-II, 1774 , p. 100 , et Bourguignat, Mal. Alg., I, 1864 , p. 288 , pl. XXXII, f. $42-46$ (Bulimus acutus Bruguières ${ }_{1789}$, Draparnaud 1805 ).

Hélice littorale commune dans toutes les régions basses de la Tunisie, sur les lierbes, les arbustes, les murailles, les rochers. C'est, avec les $H$. pisana, pyramidala, etc., une des espèces les plus répandues non seulement dans cetle partie de l'Afrique, mais encore dans toutes les contrées circumınéditerranéennes.

\section{BULIMUS.}

Les espèces de ce genre découvertes en Tunisie, au nombre de dix, sont de six groupes diflérents.

Nous ne comprenons pas dans ce genre l'anciea Bulimus, connu sous le nom de decollatus, parce que nous le classons maintenant dans la fanille des Stenogyrida.

\section{\& 1.}

B. pupa Bruguières, Hisl. Vers, in Encycl. méth. I ( $2^{\mathrm{e}}$ partie, 1792), p. 349 (Helix pupa, Linu(Pus, Syst. nat. [ed. X, 1758 ], p. $77^{3}$ ).

Ce Bulime assez communément répandu dans toute la Tunisie, où il offre de nombreuses variations de taille et de grosseur, a été constalé au pied des oliviers, sur le versant est du Djebel Ahmar près de Tunis, à Kamart sous les pierres, sur les bords de la Medjerda près le pont de Fondouk (Bourg. 1867); à la Mahamedia (Kerim, 1873); ̀̀ Bizerte, à Cartlage el aux alentours de la grolte de Hanvaria (Bellucei, 1875 ); aux environs de Kerouan (Doumel-Adanson); à la Galite (Gestro 1876 , Issel 1878 ), elc.

Nous le connaissons encore des Djebels Reças et Zaghouan, du Djebel Bou-Kourneï près Hammam-el-Lif, dı Cap Bon, d'El-Aouina, de Sidi-Daoud, de Keli bia, d'Aïı-Cherichira, du Djebel Bou-Zelfar, d'Henchir Harrath, des collines de Takrouna dans l'Enfida, de Kessera, de Zougag, d'une monlagne de grès de l'Oued Medjerda près la fronlière algérienne, des crêtes d'El-Kel', des détritus rle l'Oued Sidi-Aich, elc. (Let.).

Près de Porto-Farina, on rencontre assez fréquemuent de très grands individus, qui se rapprochent un peu du $B$. Cretensis (Berthier et Let.).

\section{s 2.}

B. Poupillierianus Bourgnignat, Mal. Alg. II, 1864 , p. 343 (B. Numidicus [non Reeve] Bourg. Nal. Alg. II, 186 , p. 15 , pl.oII, f. 29-32).

Espèce pen commune. Montagnes de grès de l'Oned Merljerda près la frontière algérienne, environs de Porto-Farina, où l'ou rencoutre parfois d'assez grands échantillons (Let.). 
\$3.

B. Micelii (Buliminus) Kobelt in Nachrichtsbl. malak. Ges. 1885, p. 115.

Ce joli Bulime, un des plus singuliers du nord de l'Afrique, signalé sur les rochers près d'Hammam-el-Lif (Kobelt), a été recueilli par M. Dounmet-Adanson, sur les pierres humides et dans les anfractuosités du Djebel Bou-Kourneïn.

Ciette espèce n'appartient point au groupe du B. Milevianus, ainsi que le pense M. Kobelt.

\section{\$4.}

B. Letonrneuxi Bourguignat, Malac. Alg.' II, p. 9, pl. II, f. 5-8, 1864.

Espèce rare; aux alentours de la source du Djebel Zaghouan (Let.).

$\$ 5$.

B. punicus Letourneux et Bourguignat, sp. nov.

Testa fere omnino obtecte rimata, subfusiformi-elongata, in medio parum ventrosa, ad basin non ampla sed potius attenuata, sat nitidula (semper inquinata), fragili, cornea, in medianis anfractibus suturam versus leviter albescente, oblique ac sat sordide striatula; spira elongala, regulariter attenuata, ad summum obtusa (apex validus, obtusus ac lævis); anfractibus 7 convexiusculis, regulariter ac lente crescentibus, sutura impressa separatis; ultimo mediocri, parum ventroso, inferne attenuato; apertura perobliqua, angusta, elongato-suboblonga, superne angulata; peristomate recto, acuto; columella brevi, basin aperturæ non attingente ac inferne retrocedente; margine columellari superne in triangulam formam dilatato ac rimam fere omnino tegente; alt. 15 , diam. 5 1/2 millim.

Le type se trouve sur les crêtes des collines d'El-Kef (Let.).

On rencontre encore cette espèce çà et lì, mais toujours peu abondante, dans le Djebel Reças et dans le Djebel Zaghouan, notamment près des rochers de la source et aux environs de Guelaat-es-Snan (Let.).

B. Milevianus Raymond in Journ. conch. p. 81, pl. III, f. 4, 1853 et Bourguignat, Malac. Alg. II, p. 7, pl. II, f. 1-3, 1864 .

Gorges des grands rochers, près Ellez (Let.). Espèce rare.

Le type de ce beau Bulime a été découvert par M. le $\mathrm{D}^{\mathrm{r}}$ Louis Raymond au sommet des rochers qui dominent Milah, petit village à 38 kilomètres 0.-N.-0. de Constantine. Depuis, cette espèce a été retrouvée vivante, en compagnie du $B$. Berthieri, avec lequel il a toujours été confondu, sur les rochers du Mécid, du Djebel Thaya et aux environs de Melila entre Batna et Constantine.

Le $B$. Milevianus, espèce fort rare et peu conmue, est une coquille, notablement ventrue inférieurement, caractérisée par une spire allongée-subconique, surmontée d'un sommet gros, obtus, comme mamelonné; les tours sont peu convexes, sauf le dernier, bien renflé et d'une taille relativement développée; 
l'ouverture suballongée-oblongue, bien arquée dı côté extcrne, est nuédiocrement oblique; la columelle rectiligne descend presque jusqu'à la base, ainsi que la dilatatiou triangulaire du hord columellaire.

D'aprè̀s l'indication de ces caractères, caractères reconnus par nous sur les échantillons types de Milah, l'on peut se rendre compte maintenant des différences essentielles qui existent entre le $B$. Milevianus et le $B$. punicus.

Le B. punicus, en effet, à l'inverse du B. Milevianus, est une espèce allongéesubfusiforme, non ventrue inférieurement, mais au contraire atténuée; son dernier tour, de moyenne taille relative, est peu convexe; son ouverture plus oblique, plns étroite par suite du peu de convexité du dernier lour, est plus alİongée; sa columelle, très rétrocédenteà la base, est plus courte, ainsi que sa dilatation triangulaire, etc.

\section{B. Berthieri Bourguignat, sp. nov.}

Testa perforata (perforatio semitecta), elongato-subconoidea, nitida, fragili, cornea, in ultimo sæpe albescente, oblique striatula (striæ passim irregulares); spira elongata, subconoidea, ad summum obtusa (apex lævigatus, submamillatus); anfractibus $7-8$ convexis, sat arcle spiratis, sulura profunda separatis; ultimo mediocri, bene convexo, inferne rolundato; apertura perobliqua, lunata, subovata, superne angulata; peristomate recto, acuto; columella relative valde retrocedente, brevi, basin aperturalem non attingente; margine columellari in triangulam forman dilatalo; ilt. 15 , diam. 6 millim.

Le type de ce Bulime vit sur les rochers, aux environs de Constantine, où il a été découvert par M. H. Berthier; depuis, celte espèce a été constatée près d'AïnMelila, au nord du Chott Mzouri, entre Constantine et Batna (Pechand) et sur les rochers du Djebel Thaya (I.el.).

En Tunisie, le B. Berthieri a été retrouvé aux alentours de Guelaat-es-Suan et dans la gorge des grands rochers, près d'Ellez (Let.).

Celte espèce est surtout caractérisée par ses tours, bien convexes, à croissance si lente que le dernier est relativement à peine plus développé que l'avant-dernier. Chre\% le $B$. Milevianus, la croissance, bien que régulière, est plus rapide, le dernier toul est plus ample, les autres tours sont moins convexes, etc.; le B. Berlhieri diffère encore du $B$. Milevianus par sa perforation non entièrement recouverte, par sa suture plus profonde, par sa columelle plus rejetée en arrière, par son outverture plus oblitique, plus grande, etc.

On peut rapporter ì cette espèce lit variélé lancéolće du B. Milevianus, représentée ( pl. Il, fig. 4) dians le tome second de la Malacologie de l'Ilgérie (1864).

S 6.

B. Jeannoti Terver cat. Moll. nord de l'Afriq. p. 30, pl. IV, f. 10-11, 1839. (B.Terveri Dupotet, mss. in Forbes, Moll. of Algiers and Bougia, in Ann. nat. hist. or Mag. of zool. etc., p. 253,1838 et pl. XII, f. 1, 1839.)

Cette espèce, assez abondamment répandue en Kalyylie, notamment aux envi- 
rons de Bougie (localité type), dans les gorges de l'Oued Isser, sur les rochers d'Albalou Toudja, etc., ainsi qu'aux environs d'Alger et même de Tlemcen, a été retrouvée en Tunisie, au Zaghouan, dans le domaine de l'Enfida, à Kessera, enfin sur les crettes d'El-Kef et aux alentours du Kef El-Djerdja (Lel.).

B. Cirtanus Morelet, in Journ. conch. p. 349, 1857, et Bourguignat, Malac. Atg. II, 1864, p. 12, pl. II, f. $15-19$.

Djebel Reças (Bell. 1875); Hammam Zeriba dans l'Enfida (Let.); environs de Kerouan (Doûm.-Ad.); El-Hadjar Mikta, Guelaat-es-Snan, Foum Goubel, Onedel-Hammam et Kef El-Djerdja (Let.).

Sur les crêtes d'El-Kef, on rencontre une variété à forme plus grêle el à tours plus bombés.

En Algérie, cette espèce est répandue dans les trois provinces.

B. charieins Bourguignat, spec. noviss. Moll. $n^{\circ} 24,1876$.

Le type a été découvert sur les crêtes des collines aux Biban, dans le Djurdjura. Ce Bulime est peu commun.

M. H. Berthjer a retrouvé quelques individus de cette espèce dans le Djebel Reças.

\section{PUPA (1).}

Les espèces de ce genre, à l'exception du Pupa granum, espèce circumméditerranéenne, sont fort rares en Tunisie; trois seulement y ont été constatées.

\section{$\S 1$.}

P. punica Letourneux et Bourguignat, sp. nov.

Testa minuta, rimata (rima semitecta), oblonga, leviter fusiformi, superne inferneque attenuata, in medio ventriculosa, sat fragili, subdiaphana, corneo-castanea, oblique striatula; spira oblonga, obtuse attenuata(apex validus, obtusus quasi mamillatus); anfractibus 5 1/2 quasi contortis, rotundatis, regulariter crescentibus, sutura profunda separatis; ultimo mediocri, parum convexo, externe ad peristoma leviter planulato, inferne attenuato, superne ad insertionem recto; apertura obliqua, subovata, sexlamellata, scilicet: lamellæ duæ parietales quarum una minuta prope insertionem, altera mediana, profundior; lamellæ duæ columellares quarum inferior minutissima et lamellæ duæ palatales sal. remotæ ac marginem non attingentes; peristomate albescente, acuto, undique leviter patulescente; margine columellari in triangulam formam dilatato ac supra rimam expanso; marginibus valde approximatis; alt. 4, diam. 1 1/2 millim.

Sur les rochers humides, près de la source, au Djebel Zaghouan(Let.).

(i) Draparnaud, 1801 (pars). 
Cette espèce, de la série du Pupa avenacea, est distincte de tous les Pupas de celte série; elle na des rapports de ressemblance au point de vue de la taille et de la forme qu'arec le $P$. Cartennensis (Letourneux, 1884) de la montagne de Tenez, en Algérie; mais celui-ci possède une ouverture complètement édentulće.

1.. Barattei Letourneux et Buurguignal, sp. nov.

Testa minuta, anguste perforata, oblongo-subconoidea, inferne non altenuata sed potius rentrosa, fragili, subdiaphana, cormea, oblique striatula; spira elongata, regulariter attenuata, nihilominus ad summum obtusa (apex perobtusus, validus, superne quasi planus); anfractibus $51 / 2$ rotmndatis, regulariter crescentibus, sutura profunda separatis; ultimo mediocri, convexo, inferne rotundato, ad insertionem reclo; apertura obliqua, subovata, quadrilamellata, scilicet: lamellæ duæ parietales punctiformes quarum superior ad insertionem, inferior profunda ac mediana; lamella columellaris unica, supera, denticulum simulans; lamella palatalis aqualiter unica, valde obsoleta ; peristomate pallicliore fragili, undique patulo; margine columellari dilatato ac expanso; marginibus approximat is; alt. 4, diam. 2 milliun.

Dans les anfractuosités des rochers, an Djebel Bou-Kourneïn (Lel.).

Ce Prnpa, de la même série que le précédent, se distingue de celui-ci non senlement par les denticulations de son ouverture, mais encore par sa forme subconoìde, ventrue à la base et non atténuée, par ses tours non torses, dont le dernier, plus convexe, est bien arrondi à sa partie inférieure.

\section{$\$ 2$.}

P. granum Draparnaud, Tabl. Moll. p. 59, 180 t, et Hist. Moll. Fr., p. 63, pl. III, ก. $45-46,1805$.

Celte espèce circumméditerranéenne parait fort répandue en Tunisir. Elle a été constatée au Bardo le long des fossés des fortifications, à Kamart sous les pierres, ì la Mahamedia (Bourg. 1867), à Sidi-Aldul-Vached (Kerim, 1873), près d'Utique dans les allurions de la Medjerda (Gestro, 1876 ), aux environs d'Hammam Zeriba dans l'Enfida (Let.), etc.; enfin, dans les détritus de l'Oued Sidi-Ä̈ch (Let.), ainsi qu'à Gafsa, à Oum-el-Lascar près le Chott El-Djerid et dans le Djebel Altig (ValeryMayet).

\section{PUPILLA (1).}

P. bigranata Locard, Prodr. Nalac. Fr. p. $17^{\text {h }, ~}{ }_{1882}$ (Pupa bigranata, Rossmässler, Iconogr. X, 1838, p. 97, f. 645).

Celte petite coquille, répandue ẹi et lì dans presque toute l'Europe centrale et méridionale, a été retrouvée, en Tunisie, dans les alluvions de l'Oned SidiAïclı (Let.).

(1) Leach, 1820 . 


\section{VERTIGO ${ }^{(1)}$.}

v. discheilia Bourguignat, Paléont. Alg. p. 78 , pl. IV, f. $3-4,1862$ et Malac. Alg. II, p. 105 , pl. VI, f. 45-47, 1864.

Cette minuscule espèce, recueillie dans les alluvions de l'Harrach près Alger, ainsi que dans celles de l'Oued Tademit (pr. Oran) et du Safsaf, près Plilippeville, se rencontre également en Tunisie, dans les détritus de l'Oued Sidi-Aïch (Let.).

V. Latasteana Letourneux et Bourguignat, sp. nov.

Testa minutissima, vix rimata, curta, lumido-ventrosa, subovato-sphærica, fragili, diaphana, cornea, lævigata; spira curta, perobtusa; anfractibus 5 convexis (embryonales parvuli, mediani relative ampli ac turgidi, ultimus minimus, diminutus ac attenuatus), celeriter crescentibus, sutura mediocriter profunda separatis; ultimo parvulo, minus ampliato quam penultimo, externe compresso, scrobiculato et ante marginem labio anteperistomali eleganter signato, ac ad basin coarctato et circa rimam angulato, tandem superne ad insertionem ascendente; apertura subobliqua, minutissima, coarctata, subtrigonali ac quinquedentata, scilicet : dentes parietales duo elongati sat validi; columellaris unicus robustus; palatales duo lamelliformes, marginem externum attingentes quorum superior validior extus scrobiculum profundum formans; peristomate pallidiore sat crasso, undique patulo; columella valde brevi; margine externo in loco scrobiculi sinuato ac antice quasi in rostrellum provecto; margine columellari dilatato; marginibus remotis; alt. 2, diam. 1 millim.

Cette infiniment petite espèce, remarquable par l'étroitesse de son ouverture, par sa forme sphérique, ne peut être assimilée à aucuns des Vertigos vivants. Nous ne voyons que le V. Milne-Edwardsi ${ }^{(2)}$ de la colline de Sansan qui puisse lui ressembler sinon au point de vue des denticulations, du moins à ceux de la taille et de la forme des contours.

\section{ISTHMIA ${ }^{(3)}$.}

1. muscorum Locard, Prodr. Malac. Fr. p. 176,188 a (Pupa muscorum, Draparnaud, Tabl. Moll. p. 56 (excl. syn.) 1801 ; Pupa minutissima, Hartmann in Neue Alpina, p. 220 , pl. II, f. 5,1821 et Dupuy, Hist. Moll. Fr. p. 422 , pl. XX, f. 13 , 1850 ; Vertigo muscorum, Michaud, Complém. hist. Moll. p. 70,1831 ).

Alluvions de l'Oued Sidi-Aïch (Let.) et de la Medjerda près Ghardimaou (Pech.).

Cette petite coquille a été constatée en Algérie dans les alluvions de l'Harrach près Alger, de l'Oued Roumana près Bou-Saada et de la Boudjimah près Bône.

(1) Müller, 1774 (par's).

(2) Bourguignat, Hist. Malac. colline de Sansan, p. $9^{2}$, f. 148-151, 188 1.

(3) Gray, 1820. 
1. Doumeti Letourneux el Bourguignat, sp. nov.

Testa minutissima, peranguste perforala, curta, cylindrica, ad summum ventricosiore quam ad basin, fragili, subdiaphana, cornea, oblique bene striata; spira brevi, ad summum perobtusa in tholum rotundata; anfraclibus 5 convexis (embryonales minuti, pallidiores), sat lente crescentibus, sulura profunda separatis ; ultimo mediocri, convexo, externe prope marginem leviter planato, ad basin altenuato, superne ad insertionem recto; apertura perobliqua, inferne retrocedente, lunata, semiovata, edentula; peristomate incrassatulo, patulescente ac albescente; margine columellari valido, reflexo; marginibus remotis; alt. 1 1/2, diam. 3/4 millim.

Cette inicroscopique espèce a été trouvée dans les alluvions de l'Oued Sidi-Aïch (Let.).

\section{CHA USITID AE.}

\section{CLAUSILIA (1).}

Longtemps on a cru que ce genre n'avait pas de représentants dans le nord de l'ifrique.

La première espèce, la Clausilia virgata, recueillie en 1847 aux environs du tombeau de la Chrétienne, à l'onest d'llger, a été inentionnée par Kuster ${ }^{(2)}$, en 1848 , sous le nom de Cl. bidens var. virgrata.

La seconde, découverte en Tunisie, a été décrite par L. Pfeiffer ${ }^{(3)}$, en 1860 , sous l'appellation de $\mathrm{Cl}$. Tristami, et reproduite sous le même vocable, l'année stivante, par Kuster ${ }^{(3)}$.

Depuis, quelques autres espèces ont été signalées aussi bien au Maroc qu’en Algérie et en Tunisie.

Voici leur liste par ordre de date:

1864, Claus. virgata et Letourneuxi, alluvions du petit ravin de Chabet Beinan, près le cap Caxines, à 11 kilomètres ouest d'Alger (Bourg. Malac. Alg. II, p. $7^{3}$ et 75$)$;

1864, Claus. Maroccana, Bourg., détritus de l'Isly (Maroc). C'est sous le nom erroné de $\mathrm{Cl}$. plicata [uon $\mathrm{Cl}$. plicala Drap. 1805] que cette espèce a été constatée par M. Morelet (in Journ. conch. 1864, p. 155 );

1868, Clans. bidens, $\mathrm{Cl}$. virgata et $\mathrm{Cl}$. panica, ruines d'Utique et de Carthage (Bourg. Malac. Tunisie, p. 29 et $3 \mathbf{1}, 1868$ );

1870 , Clans. numidica, rochers de Takouch, près Bòne (Lelourneux, Exc. Malic. in Ann. malac. 1, 1870 , p. 308);

1876 , Claus. Perinnei, Djebel Thaya, entre Jemmapes et Guelma (Lot. in Bourg. Spec. noviss. Moll. $\mathrm{n}^{\circ} 34,187^{6}$ );

(1) Draparnaud, 1805 .

(2) Gatt. Claus. (2 édit. Chemnitz), p. 54; voir égaleınent Bourg. Mal. Alg. II, p. 71, 1864.

(3) In Proceed. zool. Soc. Loud. XXVIII, 1860, p. 140.

(ง) Gatt. Claus., p. 3o7, pl. XXXV, f. 1-3, 1861 . 
1879, Claus. polygyra, ruines de Carthage (Böttger in Suites à Rossmässler, f. 1793 );

1880, Claus. Belluccï, Djebel Reças, près des carrières de plomb (Issel, Groc. del Viol. Moll. p. 102 );

Enfin, 1884, Claus. Bavayana, falaises à 18 kilomètres à l'ouest de Bône (Hagenm. in Bull. Soc. Malac. Fr. I, 1884, p. 209 ).

En somme, onze espèces qui, en réalité, se réduisent aux neuf suivantes : Cl. virgata, Tristami, Letoumeuxi, Maroccana, bidens, punica, numidica, Perinnei et Bavayana, puisque la $C l$. polygyra est une Perinnei et la $C l$. Belluccii une forme major de la Cl. Tristami.

Outre ces neuf' espèces, nous en avons trois nouvelles à faire connaître : les Cl. Bonneti, philora et Cossoni.

Cl. Bonneti Letourneux, sp. nov.

Testa angustissime rimata, subfusiformi-elongata, ad summum mamillata, parun nitente, subopacula, subcastaneo-grisea, ad partem superiorem et prope aperturam pallidiore, undique (embryonalibus 4 lævigatis exceptis) striis validis, albidis, regularibus ac relative inter se (in ultimo magis) sat distantibus, eleganter costulata; spira elongata, regulariter attenuata, prope summum gracili et cylindrica ac ad apicem turgidula (apex obtusus); anfractibus 14 fere planulatis, lente crescentibus, sutura lineari albocinctaque separatis; ultimo externo convexo, ad basin mediocriter cristato; apertura leviter obliqua, ovata, intus lutescente, ad marginem albescente, superne sinulo patulissimo signata, plicata, scilicet; A. parietales duæ quarum superior marginalis, valida, intrans, cum plica spirali non conjuncta; inferior robusla, contorta; B. plica subcolumellaris in conspectu vix perspicua, basin columellæ attingens; C. plica patatalis unica, supera, immersa, externe apparens, lunellam transiens; D. lunella litteræ T. similis, extus parum conspicua; peristomate continuo, albido, crassulo, undique patulo ac sub sinulo leviter acuto; alt. 23 , dianı. 4 millim.

Anfractuosités des rochers au Djebel Bou-Kourneïn (Let.).

Cette espèce est la Clausilie tunisienne la plus fortement costulée.

Sur un ou deux échantillons, nous avons constaté une lamelle pariétale inférieure, bipartie à sa partie supérieure, dont l'une des arêtes, invisible de face, vient se confondre avec l'autre arête juste à sa partie moyenne.

Cl. Tristami L. Pfeiffer, in Proceed. zool. Soc. Lond. p. 140,1860 el Kuster, Gall. Claus. in $2^{\circ}$ édit. Chemnitz, p. 307 , pl. XXXV, f. 1-3, 1861, et Bourguignat, Malac. Alg. II, 1864, p. 71, pl. V, f. 4-6.

Espèce assez abondante au Djebel Reças, plus rare au Djebel Bou-Kourneüı (Let.). 
Il nous a été impossible, devant les nombreuses variations de taille et de forme, d'établir une ligne de démarcation eutre cette espèce et la $\mathrm{Cl}$. Belluccï (Issel, Groc. Viol. Moll. p. $102^{(1)}$ et in Ann. mus. civ. Genova, 1880, p. 278).

Cette Cl. Belluccii constitue une variété major de la Cl. Tristami.

Chez cette variélé, la coquille est parfois ventrue avec une extrémité grêle, cyliudriforne, et d'autres fois, régulièrement effilée. Les tours varient de 15 à 19 . L'ouverture offre également des variations notables; elle est ou ovalaire, ou bien subquadrangulaire, lor'sque le côté externe prend une extension plus ou moins prononcée, ou bien encore subtrigonale-oblongue, quand le bord columellaire descend rectilignement; daus ce dernier cas, l'ouverture offre comme une apparence de sinus auprès du pli subcolumellaire.

Cette variété ( $\mathrm{Cl}$. Belluccii) est très répandue dans le Djebel Reças et dans le Zaghouan, ainsi qu’à Hammam Zeriba dans l'Enfida (Let.). Ou la rencontre encore, nuais plus rarement, au Djebel Bou-Kourneïn (Doûmet-Ad.) et à Oued-elHammam, près 'Tunis (Let.).

Cl. philora Letourneux, sp. nov.

Testa rix peranguste rimata, sat gracili, subfusiformi, elongata, ad summum cylindrica, nitida, opacula, subrosaceo-cinerea, ad superiores lutescente, ad aperturam albescente, undique (embryonalibus 4 lavigatis exceplis) eleganter striata (striæ vel potius costulæ, minuta, albidæ, regulares ac inter se vix distantes quasi conjunctæ); spira elongata, altenuala, prope summum cylindrica ac gracili, nihilominus ad apicem obtusa ac relalive sat mamillata; anfractibus 13 convexiusculis, regulariter lenteque crescentibus, sutura impressula albofilosaque separatis; ultino convexo, ad basin plus minusve cristato; apertura parum obliqua, ovata, inlus rufo-lutea, ad marginem albidula, superne non angulata (sinulus perapertus), plicata, scilicet : $\Lambda$. parietales duæ quarum superior marginalis, mediocris, cum plica spirali non conjuncla; inferior validior, contorta ac descendens; B. plica subcolumellaris in conspectu non perspicua, modo obliyue conspicua; C. palatalis una, supera, valde immersa, externe apparens et lunellam transiens; D. lunella litteræ C. inversa similis, extus perspicua; peristomate albo, reclo, parum crassulo, undique patulescente, ad marginem externum subreflexo; alt. 22, diam. 3 1/2 millim.

Le tyjpe a été recueilli sur les crêtes d'El-Kef, où il paraît assez abondant. On rencontre encore cette espèce, unais plus rarement, aux environs de Kessera (Let.).

Les striations, chez cette Clausilie, sont du doublè plus petites que celles de la Cl. Tristami; elles sont, en outre, si régulières, si rapprochées les unes des autres (sauf vers l'ouverture où elles sont un peu plus écartées), qu'elles semblent ne

(I) Du tirage à part. 
faire qu'une, bien qu'elles soient toutes très distinctes et très reconnaissables. Nous ne voyons que la $\mathrm{Cl}$. oscitans (Charp.) de l'île Lampedouse, qui puisse lui ressembler comme mode de striation.

CI. Perinnei Letourneux in Bourguignat, sp. noviss. Moll. $n^{\circ} 34,{ }^{1} 87^{6}$ (Cl. polygy'a Böttger in Suites à Rossmässler, f. $179^{3}, 1879$ ).

Le type de cette belle espèce a élé trouvé sous lés mousses et les détrilus à l'entrée d'une caverne dı Djebel Thaya, ainsi qu'au Djebel Guerar, dans la province de Constantine.

En Tunisie, cette Clausilie parait abondante dans le Djebel Zaghouan (Let. et Doùm-Ad.).

II. Böttger signale sa Cl. polygyra des ruines de Carthage. Nous doutons forl que cette espèce, qui semble une forme. rupestre, vive sur les collines de cette localité.

De même que la $\mathrm{Cl}$. Tristami, la $\mathrm{Cl}$. Perinnei offre un assez grand nombie de variations de taille et de grosseur. La plus importante est celle que nous inscrivons sous le nom de :

Var. Zaghonanica Let. el Bourg.

Celte variété se distingue dı type par sa taille un peu plus grande; par son lest, à peine fusiforme, allant en s'effilant avec régularité et plus Jonguement cylindrique à sa parlie supérieure; par ses tours tout à fait plats, séparés par une suture si superficielle qu'elle serait pour ainsi dire invisible sans son filet blanelâtre carénant; par son dernier tour comprimé sur le côlé dorsal ; enfin notamment par son ouverture différente (denticulations à part); celle-ci, en effet, plus oblique, de forme subtrigonale, est sensiblement rétrocédente à la base; son côlé externe, plus rejeté en arrière, possède un bord plus réléchi et plus dilaté; son sinus supérieur est moins ouvert; enfin, son côté columellaire, au lieu d'être arqué, comme chez le type, est rectiligne, ce qui donne lieu à une apparence d'angulosité à la partie inférieure.

Cette variété semble assez répandue dans le Djebel Zaghouan (Let.).

s 2.

Cl. Cossoni Letourneux, sp. nov.

Testa anguste rimata, elongata, parum ventrosa, nitida, opacula, uniformiter castanea, ad suturam in medianis eleganter albo-papillifera, subtiliter striatula (striæ obsolèlæ; ad aperturam bene distincta ac leviter validiores); spira altenuata, ad summum truncata; anfractibus 9 (enbryonales absunt) convexiusculis, lente crescentibus, sutura inpressula, inter médianos papillifera, inter ultimos simplici aut leviter albo-filosa, separatis; ultimo convexo, inferne obluse cristato; apertura bene obliqua, ovata, intus castinea, ad marginem luteo-albescente, plicala, scilicet: A parietales duæ quarum superior marginalis, producta, valida, profunde intrans, cum plica spirali non conjuncta; inferior contoria, descendens; 
B plica'subcolumellaris minuta, conspicua; $C$ palatales duæ quarum una supera elongata; altera infera; brevis, valde immersa; D plica interparietalis denticulo similis, profunda, parietalis inferæ proxima; E lunella litteræ I similis, extus apparens; peristomate continuo, luteo-albescente, valido, crasso, undique expanso ac subreflexiusculo, sub sinulo aperturali magis incrassato; alt. 18 , diam. 4 millim.

Dans les anfractuosités des rochers, au Cap Bon (Let.).

Cette espèce appartient à la série des Cl. Bavayana (Hagenm.) et numidica (Let.) du massif de l'Edough, près de Bỏne.

C1. punica Bourguignat, Malac. Tunis, p. 3o, fig. $34-37,1868$.

Excessivement commnne sur les ruines d'Utique (Bourg. 1867).

Cl. virgata Cristofori et Jan, cat. rèr. natur. II, p. 5, 183 2 (Clansilia papillaris, var. virgata, Rossmässler, Iconogr. III, 1836 , p. 12, f. 170 ).

Ruine des anciens bains romains près de la source thermale à Utique (Bourg. 1867); rochers près de Porto-Farina; collines de Carthage et à El-Aouina (Let.).

C. bidens Turton, Moll. Brit. p. 73 , f. 56, $183_{1}$ (Turbo bidens Linnous, syst. nat. [ed. X] I, P. 767,1758 , et [ed. XII] p. $\left.1240,{ }_{7} 7_{7}\right)$.

Sous les pierres, à Carthage (Bourg. 1867), peu abondante.

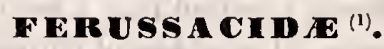

Autrefois, on regardait comme des Helicidæ toute la série européenne des coquilles achatiniformes que nons classons actuellement dans une famille particullière.

Les savants travaux anatomiques de MIM. Watson, Godwin-Austen, Nevill, etc. sont venus inontrer que les espèces achatiniformes (à l'exception de celles comprises parmi les Zuas et les Azecas de la section des Azecastrum) ${ }^{(2)}$ étaient pourvues d'un pore muqueux.

J.es genres que nous comprenons dans celle famille sont:

$1^{\circ}$ Ferussacia (Risso ${ }_{1826}$ [non Ferussacia Leufroy ${ }_{18.28} 8$, nec Ferussina Grateloup 1827]);

$2^{\circ}$, Ilypnophila (Bourg. 1858 in Rev. et mag. zool. in Amén. mal. II, p. 88, 1859), genre Cryptazeca de Folin, 1877 .

Le type spécifique de ce genre est l'ancien Azeca pupaformis ;

$3^{\circ}$ Calaxis (Bourg. in sched. 1882). Genre établi pour un petit groupe d'espèces syriennes caractérisées par une columelle pourvue inférieurement d'une forte

11: Bourg. Hist. Malac. Abyss., p. $120,1883$.

(2) Les Mollusques des genres Zua et Azeca (sec. Azecastrum) sont saus pore inuqueny. 
lamelle formant rampe et obstruant la base de l'ouverture. Les Calaxis; tels que les C. Hierosolymarum, Rothi, Saulcyi et Moussonianus, ont tous été décrits et figurés. (jl. XIX) dans la $4^{\circ}$ décade $(1864)$ des Mollusques nouveaux.

$4^{\circ}$ Hohenwartlia (Bourg. 1864 in Moll. nouv. [ [ $4^{\mathrm{e}}$ déc.], p; 119 ). Coupe sousgénérique établie autrefois pour les petites Ferussacies ayant l'apparence de Cæcilianelles;

$5^{\circ}$ Calestele (Benson $1864^{\prime}$ in Ann. and mag. nat. hist. ( $3^{\circ}$ série), ẊIII, p. 135). Francesia (Paladilhe in Ann. mus. civ. Genova III, p, 10, 1872). Ce genre comprend donze espèces répandues dans l'Inde, l'Arabie, l'Égypte et l'Espagne.

Il convient de placer encore dans cette fanille le genre Glessula (Martens), établi par une espèce abyssinienue ${ }^{(1)}$ et les genres Agraulina et Alsobia ${ }^{(2)}$, créés jadis comme coupes sous-génériques des Azecas ponr deux séries d'espèces des illes Madère et Clanaries.

M. Watson a depuis proposé pour les Agraulina le nouveau nom de Lowea ${ }^{(3)}$.

\section{FERUSSACIA (1).}

Ce genre, tel qu'on doit le comprendre actuellement, se compose des espèces des anciens groupes Fnlliculiana et Proceruliana ${ }^{(5)}$. On doit rejeter de cette coupe générique les anciennes formes publiées sous les noms de Fer. subcylindrica (Zua lubrica des auteurs); azorica, Maderensis, exigua, etc. pour les réunir dans un genre à part, celui des Zuas de Leach $(1820)$; entin les Hohenwarthia, ainsi que nous l'avons dit plus haut, parce qu'elles doivent constituer une coupe générique spéciale.

Les Ferussacia, au nombre de cinquante-sept espèces, peuvent se répartir en trois grandes séries: $1^{\circ}$ en espèces Folliculiennes, $2^{\circ}$ en Fausses Folliculiennes et $3^{\circ}$ en Procérulicnnes.

1 Les Folliculiennes, dont tous les malacologistes connaissent les caractères, puisqu'elles ont pour type la $F$. folliculus, sont répandues dans toutes les contrées occidentales dı bassin de la Méditerranée. Treize espèces : les $F$. folliculıs, regularis, gravida ${ }^{(6)}$, amblya, amauronia, Thayaca ${ }^{(7)}$, Vescoi, Forbesi, Gronoviana, proechia, aphelina, ruricola et abromia;

$2^{\circ}$ Les Fausses Folliculiennes, espèces ayant l'apparence des Folliculiennes, tout en possédant uı̣e ouverture lamellifère et un bord columellaire similaire à celui

(1) Voir-Bourg. Mal. Abyss, p. $120,1883$.

(2) Bourg. 'in Rev." et mag. zool. 1858 et Amén. mal. II (janvier 1859 ), p. 88.

(3) Watson, Some notes on the Maderian Moll. ident. by the Rev. R. T. Lowe as Achat. folliculus. 1875 .

(9) Risso, Hist. nat. Eur. mérid. IV, p. 80,1826, et Bourg. Amén. mal. I, p. 197, 1856 et Mal.

Alg. II, p. 23,1864 .

(3) Voir Bourg. Mal. Alg. II, 1864 et Moll. nouv. (4 déc. 1864).

(6) Florence, sp. nov. Belle espèce nouvelle du déparlement du Var.

(9) Bourgnignat, sp. nov. Espèce du Djebel Thaya, dans la province de Constantine. 
des Piocéruliemes; trois espèces, toutes'de la Tunisie, les $F$. splendeus, obesa et lamellata;

$3^{\circ}$ Les Procéruliennes, dont les caractères sont également bien connus, sont des Ferussacies spéciales au nord de l'Afrique (Maroc, Algérie, Tunisie), où on les rencontre presque partout; elles se divisent en plusieurs groupes :

A. En I'raies Procérulicnnes, caractérisées par des tours assez convexes, une sutıre prononcée, une ouverture édentulée, une columelle courte, droite, délicate el inférieuremeut acuminéc, enfin par un bord externe mince et une croissance spirale régulière, sauf chez la $F$. procerula, où cette croissance est un tant soit peu plıs rapide à partir de l'avant-deruier tour; trois espèces, les $F$. proeerula, littoralis et eulissa;

$B$. Eil Alexiformes, dont on ne connaît actuellement qu'une espèce, la $F$. Nympharum, qui a une certaine ressemblance avec l'Alexia myosotis;

C. En IIfrennülleriennes, remarquables par leur coquille oblongne assez allongéé, à tours à peine convexes, à suture superficiclle et à croissance spirale régulière, ainsi que par leur onverture. édentuléc on lamellifère, par leur axe colunellaire couri, relativenent robuste et par leur hord externe un pen épais; quatre especes, les F. Magenmülleri, cremiophila, montana et Oranensis;

D. En Puniques ou Carnéennes, qui se distinguent par un test relativement opaque, une ouverture fortement lamellifère, un bord externe épais, une forte callosité el par une croissance spirale assez régulière, sauf chez la $F$. lancllifeı $a$. Huit espèces qui sembleut spéciales à la Tunisic et à la province de Constantine ${ }^{(1)}$, les F. earnea, stenostoma, gibbosa, punica, polyodon, lanellifera, Maresi et Doumeti;

E. Eı Charopiennes, caractérisées par une coquille allongée, à croissance spirale r'igulière et relativement lente; cint espèces, les $F$. Dactylophila, Barattei, charopia, Lallemanti et Pechaudi ${ }^{(2)}$ :

$F$. En Grêles ou Graeilenticnnes, distinguées par une cợuille plus on moins allongée, à test toujours mince, fragile, et par une croissance spirale non réguliere, mais plus ou moins rapide, selon les espèces; cinq Ferussacies, les $F$. gracilenta, diodonta, subgracilenta, abia et Teveri;

$G$. En Letourneuriennes, remarquables par le brillant de leur coquille, par leur spire brusquement atténuée et leur croissancé spirale fort irrégulière. C'est dans ce groupe que se trouve le géant du genre, la magnifique $F$. Letourneuxi, du sommet du Djebel Guerioum, au sud de Constintine; dix espèces, les F. Lctonrneuxi, Aristidis ${ }^{(3)}$, Berthieri, microxia, celosia, sciaphila, Cirtana, cunychia, Mabilliana el debilis;

(1) Une d'elles a été acclimatéc dans l'ìle Pianosa, près des cótes de la Toscane.

(2) Bourguignat, sp. nov. Espece de la province de Constantine.

(3) Buurguignat, sp. nov. Espèee des Djebel Guerioum et Maadid, dans la provinee de Conslantine. 
II. En Belloiriennes, caractérisées par leur coquille ovuliforme; cinq espèces, les $F$ : Belloiri ${ }^{11}$, Cossoni, mumidica ${ }^{(2)}$, Atlasica ${ }^{(3)}$ et scaptobia.

Sur ces cinquanle-sepl espèces, les trente-deux suivantes ont été constatées en Tunisie:

\section{s 1.}

F. folliculns Bourguignat in Amén. malac. I, p, 197 (en nòte), 1856 et Chât. d'If', p. 23 , pl. II, f. 1-3, 1860 (Helix folliculus, Gronovius, Zoophyt. III, p. 296 , pl. XIX, f. 15-16, 1781 ; Achatina folliculus, Lamarck, 18 22 ).

Utique, Porto-Farina, Cartlıage et dans les alluvions de l'Oued Sidi-Aïch (Let.).

F. regularis Bourguignat, Malac. Chàt. d'If, p. 20, pl. II, f. 8-9, 1860.

Alluvions de l'Oued Sidi-Aïch (Let.).

F. Veseoi Bourguignat, in' Amén. malac. I; p. 203, 1856 et Malac. Ch. d'Ir, P. 23 , pl.1I, f. 1 0-13, 1860 (olim Glandina Vescoi, Bourguignat, 1856 et Achatina Vescoi, Pfeiffer, 1859).

Environs de Tunis (Bourg. 1867); la Mahamedia (Gestro); El-Aouina et alluvions de, l'Oued Sidi-Aich (Let.), ainsi qu'à Tozer (Kerim 1873 ).

F. Forhesi Bourguignat, in Amén. malac. I, p. 204,1856 (Achatina nitidissima [non Krynicki, 1833], Forbes 1838 ; Achatina Forbesi Pfeiffer, 1859).

Grêtes du Kef (Let.).

F. proechia Bourguignat, Nalac. Alg. II, p. 44, pl. III, f. $26-28,1864$ el in Moll. nouv. ( $4^{\circ}$ décade 1864$)$, p. 124.

Crêtes du Kef, environs de Galsa et alluvions de l'Oued Sidi-Aïch (Let.).

F. abromia Bourguignat, Malac. Alg. II, p. 45, pl. III, f. 29-31, 1864 ẹt in Moll. nouv. ( $4^{e}$ déc. 1864 ), p. 125.

Sous les pierres, près la source thermale d'Utique (Bourg. 1867); sous les détritus et les bois pourris au Djebel Reças (Let.).

\section{$\$ 2$.}

F. splendens Bourguignat, sp. nov.

Testa relative sat curta, nihilominus oblonga, subventrosa, nitida, pallide cornea, argutissime substriatula aut subpolita; spira mediocriter producta, ad summum sat abruple attenuata (apex obtusus); anfractibus 7 subconvexiusculis, subirregulariter (superiores lente, cæteri rapidius) crescentibus, sutura lineari pallidioreque separatis; ultimo mediocriter majore, dimidiam altitudinis nou aquante, convexiusculo; apertura obli-

(I) Letourneux, sp. nov. Espéce des environs de Guelma.

(2) Bourguignat, sp. nov. Espcee d'Aïl-Melila, dans la province de Constantine.

(3) Bourguignat, sp. nov. Espèce du petit Allas de Blidah. 
qua, ad basin retrocedente, sat lunata, irregulariter oblonga, superne angulata, externe subarcuata, intus in ventre penullimi lamellifera; columella valida, recte descendente, inferne acuta, callosa "ac superne quasi subdenticulata; peristomate recto, obluso, incrassatulo; margine externo superne arcuato ac provecto, ad basin retrocedente; marginibus

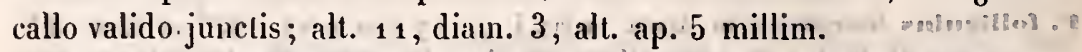

Alluvions de la Medjerda, près le pont de Fondouck, entre Tunis et Utique (Bourg. 1867).

Au point de vue de la forme, cetle éspece ressemble un peu à la $F$. aphielina.

F. obesa Letourneux et Bourgnignat, sp. nov.

Testa tumido-oblonga, obesilormi, nitidissima, pallide cornea, ad suturam albo-filosa, polita; spira mediocriter producta, ad summum șat $_{\text {a }}$ rapide attenuata (attenuatio regularis; apex validus, oblusus); anfractibus 6 vix convexiusculis, regulariler usque ad penultimum, 'dein rapidius (sutura penultimi relative sat descendens) crescentibus, sutura lineari et albo-filosa separatis; ultimo relative mediocri, tertiam partem altitudinis leviter superante, convexiusculo; apertura subobliqua, pyriformi, superne perangulata, lamellifera (lamella minuta, valde immersa, in plicam intus in ventre penultimi prolongata); columella brevi, parum crassa, recte in direclionem subdextrorsus obliquam descendente; peristomate recto, obtusato, albescente, quasi continuo (margines callo vulido juncti); margine externo suboblique recte descendente; alt. 12 , diam. 4, alt. ap. 4 1/2 millim.

Espèce rare. Comme forme, cettc Ferussacie rappelle la $F$. Gronoviana; elle vit dans le Djebel Reças (Lct.).

\section{F. Iamellata Bourguignat, sp. nov.}

Testa elongala, fragili, translucida, nitida, subalbescente, persubtiliter striatula, quasi polita; spira producta, ad summum obtuse attenuata (apex obtusus); anfractibus 6 vix convexiusculis (penultimus convexior), lente usque ad penultimum, dein velociter crescentibus, sutura impressula separatis; ultimo mediocri, tertiam parten altitudinis xquante, ad initium vix convexiusculo, ad aperturam convexiore; apertura verticali, sat lunata, irregulariter oblonga, externe arcuata, superne angulata, ii ventre penultimi valide lamellata; columella recta, acuminata, superne intus quasi subdénticulata; peristomate recto, acuto ; margine exterilo leviter arcuato; marginibus tennissimo callo junctis; alt. 10, diam.. 3 , alt. ap. 3 millim.

Cette espèce, dont le type a été conslaté aux cnvirons de Boghar, dans la province d'Alger, a été retrou vée sur les ruines de Carthage (Bcrthier); elle rappellc par sa forme la $F$. proechia. 


\section{§ 3 .}

F. procerula Bourguignat, in Amén. malac. I, p. 198 , pl. : XIX, 7-9, 1856 et Malac. Alg. II, p. 46, pl. IV, f. $37,7,1864$. (Glandina procerula Morelet; in Journ. conch. p. 357, pl. IX, f. 12,1851 ).

Espèce rare en Tunisie ${ }^{(1)}$, un seul échantillon recueilli dans le. Djebel Reças (Let.).

Dans l'Algérie, cette Ferussacie est abóndante aux alentours de la Callé, de Bỏne, d'Hammam-Meskhoutin, de Constantine et même d'Alger.

\section{F. littoralis Bourguignat, sp. nov.}

Testa elongata, nitida, subpellucida, pallide cornea, polita; spira elongata, sat regulariter attenuata (apex obtusus); anfractibus 7 convexis, regulariter crescentibus, sutura relative profunda separatis; ultimo tertiam partem altitudinis superante, convexo; apertura subobliqua, oblongopyriformi, superne acute angulata, intus profunde lamella minutissima", modo oblique conspicua, ornata ; columella relative b́revi, mediocri, acuminata, leviter curvata, superne intus denticulata; peristomate recto, subaculo, intus incrassatulo ac subalbescente; margine externó reguláritér arcuato; marginibus tenui callo junctis; alt. 13 , diam. 4 , alt. ap. 5 millim.

Ceite belle espèce, remarquable par la régularité de sa croissance spirale et par la convexité de ses tours, a été recueillie dans les détritus de l'Oued Sidi-Aïch (Let.).

Le type vit aux environs de là Calle, ainsi qu'aux alentours dé Bône (Let.).'

F. eulissa Letourneux in Bourguignat, Spec. noviss. Inoll. $\mathrm{n}^{\circ} .41,1876$.

Cette Ferussacie algérienne, constatée au Djebel-Thaya, ainsi qu'à HamınamMeskhoutin et dans le ravin du Rummel, près de Constantine, a été également trouvée en Tunisie, dans les détritus de 'l'Oned Sidi-Aich (Let.), ' où elle semble rare.

\section{S 4 .}

\section{F. Nympharum Letourneux et Bourguignat, sp. nov.}

'Testa oblongo-elongata, mediocriter nitida ac pellucida, saturate cornea aut subolivacea, ad peristoma luteola et ad summum pallidiore, polita; spira oblonga, regulariter attenuata (apex obtusiusculus); anfractibus 7 vix convexiusculis, regulariter, ac nihilominus sat velociter crescentibus, sutura lineari separatis; ultimo sat magno, dimidiam alti-" tudinis fere aquante, convexiusculo; ape rtura leviter obliqua; elongata,

(1) C'est a tort, crojons-nous, que N. le docteur Morlet (iii Arch. miss, sc. $3^{e}$ série, VII, 1881, p. 395 ) a signalé cetté espéce à Krriz, dans le sud de la Tunisic. 
superne acute angulata, inferne ad basin columellæ quasi angulosa, externe arcuala, intus subalbescente ac profunde in ventre penultimi lamellata (lamella minuta); columella valida, crassa, recte descendente, superne subdenticulata, inferne subtruncatula; peristomate recto, obtusiusculo, inlus incrassatulo; margine exlerno vix arcuatulo; marginibus tenui callo junctis; alt. 13 , diam. 4 , alt. ap. 6 millim.

Dans le temple des Nymphes, an Djebel Zaghouan (Let.), où cette espèce parait rare; endroits ombragés, au Djebel Bou-Kourneün, où elle est également peu commune (Doủm.-Ad.).

\section{\$ 5 .}

F. Hagenminlleri Bourguignat, sp. nov.

Testa elongata, nitida, subopacula, cornea aut aliquando corneoluteola, polita; spira elongato-oblonga ac regulariter attenuata (apex oblusiusculus); anfractibus 7 vix convexiusculis, regulariter crescentibus, sutura lineari pallidioreque separalis; ultino mediocri, convexiusculo, tertiain parten altitudinis superante; apertura verticali, lunata, irregulariter subovata, superne angulata, intus abescente ac in ventre penultimi minute lamellata; columella recta, valida, alba, acuminata, nihilominus inferne subtruncatula, intus quasi uni- aut bidenticulata; peristomate recto, acuto, intus albo-incrassatulo; margine externo regulariter arcuato; marginibus tenui callo junclis; alt. 13 , diam. 4, alt. âp. 5 millim.

\section{El-Aouina , Djebel Bou-Kourneïn et Guelaat-es-Snan (Let.).}

Au Djebel Reças (Let.), on rencontre une forme d'une taille un peu plus forte et d'une belle couleur cornée jaune orangrée.

Le type de cette Ferussacie, dédiée au savant docteur Hagagnmüller, a été découvert à Roknia, près Hammam-Meskhoutin, ainsi qu'an Djebel Thaya et dans les alluvions de la Seybonse, près de Bône. Nons connaissons encore cette espèce du ravin du Runumel, près Constantine, du Djebel Maadid, entre le Hodna et la Medjana, des environs de l'Aïn Guergour, dans une gorge du Bou-Selanı, à 40 kilomètres N.O. de Sétif, enfiı des alentours de Sairla, dans la province d'Oran.

F. eremiophila Bourguignat, in Ámén. malac. I, p. 199, pl: XIX, f. 20-23, 1856 et Valac. Algér. II, p. 48, pl. IV, f. 1-4, 1864.

Cette Ferussacie, si répandue dans la province de Constantine, existc en Tunisie aux environs de Porto-Farina (Berthier) et au Djebel Reças (Let.).

F. montana Bourguignat, sp. nov.

Testa oblongo-tumida, nitidissima, pellucida, cornea, polita; spira oblonga, regulariter attenuala (apex obtusiusculus); anfractibus 7 convexiusculis, regulariter usque ad, penultimum, dein rapide cres- 
centibus, sutura lineari, in penultimo valde descendeule, separatis; ultimo magno, dimidiam altitudinis fere æquante, relative sat convexiusculo, superne lente descendente; apertura fere verticali, oblongo-pyriformi, superne acute angulata, in ventre penultimi valide lamellata; columella brevi, albescente,' acuminata, superne intus subdenticulata; peristomate recto, acuto, incrassatulo ac albescente; margine externo regulariter arcuato; marginibus callo valido junctis; alt. 15 , diam. 5 , alt. ap. 7 millim.

Le type vit au Djebel Maadid, entre le Hodna et la Medjana, ainsi qu'au sommet du Mahmel $\left(2,306^{\mathrm{m}}\right)$, dans l'Aurès (Let.).

En Tunisie, cette Ferussacie a été retrouvée typique au Djebel Reças', et d'une taille un peu plus faible au Zaghouan, au Djebel Bou-Kournën, ainsi qu'à Moktael-Hadjar, à El-Aouina, à Guelaat-es-Snan (Let.), enfin aux ruines d'Utique (Bourg. 1867 ), où elle parait fort rare.

\section{\$6.}

F. carnea Bourguignat, Moll. Alp.-Marit., p. 52, pl. I, f. 23-25, 1861 et Malac. Algér. Il, p. 5o, pl. III, f. 32-35, 186 亿 (Pegea carnea Risso, Hisl. nat. Nice, IV, p. 88, fl. III, f. 30 (mala), 18 86 ).

Cette espèce est la Ferussacie la plus répandıe en Tunisie; on lá rencontre presque partout dans la région de Tunis, à Carthage, à Hammam-el-Lif, PortoFarina et Utique, où elle est des plus communes; elle a encore été constatée au Djebel Reças, au Djebel Bou-Kourneïn, au Zaghouan, près le temple des Nymphes, ainsi qu'à Kessera et à Oued-el-Hammam (Let.). Dans le domaine de l'Enfida, elle vit à Hammam Zeriba, à Dạr El-Aroui et sur les collines de Takrouna (Let.).

En Algéric, nous la connaissons d'Aïn-Melila au sud de Constantine, et, en Italie, de l'ile Pianosa, sur les côtes de la Toscane.

\section{F. stenostoma Bourguignat, sp. nov.}

Testa oblongo-subfusiformi, parum nitida, subopacula, in ultimo prope aperturam crasso-opaca; cornea, persubtililer striatula aut quasi polita ; spira oblonga, ad summum sat abrupte altenuala (apex minulus, nililoninus obtusiusculus); anfractibus 7 vix subconvexiusculis, regulariter lenteque usque ad penultimum, dein rapide crescentibus, sulura lineari separalis; ultimo majore, convexiusculo, inferne leviler allenualo; apertura verticali, anguste elongato-oblonga, superue inferneque angulata, intus albescente ac in ventre penultimi ralide lamellata; columella valida, recta, lamellosa, acuminala, nihilominus ad basin subtruncalula, intus superne subdenticulata; peristomate recto, oblusalo, intus incrassatulo ac albescenle; margine externo regulariter subarcuato; marginibus callo junctis; alt. 13 , dian. 4 , all. ap. 5 1/2 millim. 
Ruines d'Utique (Bourg. 1867); Djebel Zaghouan, près du temple tles Nymphes, où un seul exemplaire a été trouvé (Let:).

\section{F. sibbosa Bourguignat, sp. nov.}

Testa obeso-oblonga, sat tumida, sinistrorsus quam dextrorsus convexiore ut apud $F$. Gronovianam, nitida, subpellucida, cornea, polita; spira obesa, parum producta, sat velociter attenuata (apex obtusiusculus); anfractibus 7 convexiusculis (convexitas sinistra leviter validior quan dex(ra), sat lente usque ad penultimum, dein rapide crescentibus, sutura lineari albescenteque separatis; ultimo magno, dimidian altitudinis æquante, sat bene convexo; apertura verticali, irregulariter oblonga, intus albescente ac in ventre penullini valide lamellata; columella valida, brevi, alba, acuminata, intus superne sat valide denticulata; peristomate recto, acuto, iutus albo-incrassato; margine externo fere recte descendente, ad basin leviter retrocedente; marginibus callo valido junctis; alt. 11 , diam. 4, alt. ap. $51 / 2$ millim.

Environs de Bizẹte (Berthier) et Djebẹl Reças (Lẹ.); espèce rare.

\section{F. puniea Bourguignat, sp. nov.}

Testa cylindriforni-elongata, superne sat abrupte attenuata, inferue ad marginem externo-aperturalem sat valide convexa et relative dextrorsus prodeunte, nitidula, subopacula, ad aperturam et ad basin ultimi crassa, cornea, polita; spira elongata, cylindriformi, ad summum sat abrupte attenuata (apex exiguus, nihilominus obtusiusculus); anfractibus 7 vix convexiusculis, vel potius subplanulatis, sat rapide regulariterque crescentibus, sutura lineari separatis; ultimo mediocri, dextrorsum prope marginem externum bene convexo, inferne ad columellam valde opaco ac quasi suberistato; apertura subverticali, irregulariter oblonga, superne acula, intus albida ac in ventre penultimi valide lamellata; columella recta, valida, tum acuminala tum ad basin truncatula, iıtus superne denticulata; peristomate albesceute, recto, obtusato, inferne leviter subpatulescente, intus incrassato; margine externo fere recto, inferne retrocedente; marginibus callo valido junctis; alt. 14 , diani. 4 , alt. "1p. 5 millim.

Ruines d'Utique et de Carthage (Bourg. 1867), Djebel Reças et Djebel Zaghouan (Let.), Bjebel Bou-Kourneïn (Doûm.-Ad.), enfin çà et là, dans le domaine de l'Enfida (Let.).

En Algérie, la présence de cette espèce a été conslatée aux environs de Constantine.

F. polyodon Letourneux et Bourguignat, spec. nov.

Testa elongata, leviter sinistrorsus quam dextrorsus convexiore, nitida, 
opacula, in ultimo opaciore, pallide cornea , subtiliter: striolata, nihilominus polita; spira producta, regulariter attenuata (apex minutus, 'obtusiusculus); anfractibus 7 sat convexiusculis, regulariter crescentibus, sutura impressula, nihilominus inter ultimos sublineari, separatis; ultimo relative mediocri, quartam partem altitudinis leviter superante, convexiusculo, ad basin crasso; apertura verticali, irregulariler oblonga, tridenticulata; quorum unus lamelliformis in ventre penultimi'; secundus denliculiformis ad partem superam columellæ et tertius validus tuberculiformis in pariete externo ac cum lamella palatali oppositus; columella recta, robusta, crassa; peristomate 'recto, obtuso, incrassalo, quasi labialo; maroine externo in medio vix súbarcuatulo; marginibus valido callo junclis; alt. 13 , diam. 4 , alt. ap. 5 millim.

Djebed Reças (Let.).

\section{F. Maresi Bourguignat, sp. nov.}

Testa oblonga, sat curta, nitidissima, pellucida, cornea, polita; spira parum producta, suboblonga, sat abrupte truncata et ad summun gracili quasi subcylindriformi (apex minutus, obtusiusculus); anfractibus 7 convexiusculis (superiores 4 exigui ac arcle spirati, quintus major et cæteri relative maximi); sutura pallidiore linearique separatis; ultimo magno, dimidiam altitudinis æquante, convexiusculo; apertura verticali, oblonga, superne perangulata, ad basin marginis externi sat ampliata; intus albescente ac bilamellata, quarum una valida lamelliformis prope columellam et altera denticuliformis ad partem superam columellæ; columella brevi, alba, rëcta, acuminata; peristomate reclo, obtusiüsculo, inlus incrassato ac albescente; margine externo valde antrorsum arcuato; marginibus valido callo junctis; alt. 11 , diam. $3,1 / 4$, alt. ap. $51 / 2$ nillim.

Détritus de l'Oued Sidi-Aïch (Let.).

Le type de cette belle Ferussacie, dédiée au docteur Paul Marès, vit aux environs d'Aïn-Melila, au nord du Chott Mzouri, ainsi qu'aux alentours de Bône, et de Constantine.

\section{F. Doumeti Bourgitignat, sp. nov.}

Testa ventrosa," sat curla, oblongo-oviformi, nitida, subpellucida, cornea, argutissine striatula aut polita; spira parum producta, abruple attenuala el ad summum velociler acuminata (apex minutissimus); anfractibus 7 leviter convexiusculis (supcriores 4 exigui, arcte spirati [sicut in $F$. Maresi],"quintus major' et ventrosus, cæteri ampli oblongique), sutura lineari, in ultimo sápe albo-filosa, scparatis; ultimo magno, dimidiam altitudinis fere æquanle, convexiusculo; apertura verticali, oblonga, superne angulata, inlus albidula et lamellifera (lanella una valida in ventre penultimi); columella brevi, crassa, candida, recla, acuminata, 
intus superne valide denticulata (denticulus modo oblique conspicuus); peristomate recto, obtuso, intus albo-incrassato; margine externo vix arcuatulo; marginibus callo junctis; alt. 13 , diam. 5 , alt. ap. 6 millim.

Cette espèce, à laquelle nous attribuions le nom de M. Doukmet-Adanson, vit sur le Djebel Bou-Kournein, ainsi qu'à El-Aouina et à Carthage.

Au Djebel Bou-Kourneïn, où se trouve le type, on rencontre souvent une forne minor de cetle espèce.

\section{\$ 7.}

F. Dactylophila Issel, Croc. del Violante, Moll. p. $9^{8}$ et in Anu. mus. civ. Genova, p. 274,1880 (Ferussacia Isseli, Bourg. olim in sched.).

Le type de cette belle espèce a été découvert dans l'oasis de Gafsa (Kerim. $1873)$.

('ette espèce, qui semble assez abondante dans la Régence, a été conslatée, en outre, an Djebel Bon-Hedına, entre Sfax et Gafsa (Doùm.-Ad.), dans les détritus de l'Oned Sidi-Aich, à Mokta-el-Hadjar, à l'amphithéâtre d'El-Djem, an Djebel Zaghouan, sur les crêtes du Kef (Let.), enfin à Carthage (Pechaud).

En IIgérie, nous la connaissons d'El-Kantara, près Biskra, et de Boghari, près Boghar.

F. charopia Bourguignat, Malic. Ny. II, p. 54, pl. IV, f. 6-10, 1864.

lispèce assez abondante en Tunisie, où elle a été constatée sur les crêtes du Kef, à Mokta-el-Hadjar, à El-douina, à Thelepte près de Feriana, sur les rochers an bord de l'Oued Zegzaou, enfin dans les détritus de l'Oued Sisli-Aïch (Let.) et à Keriz (André).

Eu Algérie, ta F. charopia est abondante à Aün-Smeida et sur les Rochers de Sel, près Djelfa (localité type), sur le Kef-Ir'ghoud, au sud de Teniet-ei-Haad, à Sebain Aioni, sur le phateau du Sersou, aux environs de Daya (prov. d'Oran), enfin au Djebel Thaya, dans la province de Constantine.

\section{F. Barattei Letourneux et Bourguignat, sp.' nov.}

Testa oblonga, fragili, pellucida, nitida, cornea, polita; spira sat producta, iu oblongam formam attenuata (apex obtusus); anfractibus 7 vix subconvexiuscnlis, lente usque ad quintum, dein leviter rapidius, crescentibus, sutura lineari pallidioreque separatis; ultimo inajore, convexiusculo, dimidiam altitudinis fere æquante; apertura verticali, oblonga, superne perangulata, in conspectu edentula, sed oblique lantellifera (lamella stricta, valde immersa); columella brevi, gracili, acuminata, subrecla aut subcurvata, superne intus denliculata (denticulns modo oblique perspicuus); peristomale recto, acuto; margine externo antrorsum parum arcuato; marginibus tenuissimo callo junctis; alt. 11, diam. 3 , alt. ap. 5 millim.

Aïn-Cherichira, amphithéâtre d'El-Djem et Kef El-Djerdja (Let.). 
F. Lallemantl Bourguignat, sp. nov.

Testa gracili, elongata, leviter cylindriformi, fragillima, pertranslucida, fere albescente ac polita; spira elongata, regulariter attenuata, ad summum obtusiuscula; anfractibus 7 convexiusculis, regulariter crescentibus, sutura lineari, nihilominus leviter impressula, separatis; ultimo mediocri, parum convexiusculo, $1 / 3$ altitudinis æquante; apertura verticali, oblonga, in ventre penultimi minute lamellata; columella gracili, rectå, acuminata; peristomate recto, cultrato; margine externo fere recte descendente, ad basin nihilominus leviter retrocedente; marginibus diaphano callo, vix conspicuo, junclis; alt. 10 , diam. 3, alt. ap. 3 1/4 millim.

Le type de cellte espèce, dédiée à M. Ch. Lallemant, vit aux environs de Boghar.

En Tunisie, celte espèce a été retrouvée à Guelaat-es-Snan et à Foum-Goubel (Let.).

\section{S 8.}

F. abia Bourguiznat, Malac. $A l g$. II, p. 65 , pl: IV, f. $31-34,1864$.

Denx écliantillons bien typiques, recueillis dans les détritus de l'Oued Sidi-Aich (Let.), suffisent pour constater la présence en Tunisie de cette espèce si commune en Algérie.

\section{§.}

F. Berthieri Bourguignat, sp. nov.

Testa oblonga, ad summum valde attenuata, nitidissima, subpellucida, ad aperturam opaca ac sat crassa, polita, pallide cornea aut lactescente; spira mediocriter producta, fere subito altenuata ac ad summum graciliore et subacuminata (apex nihilominus obtusiusculus); anfractibus 7 (quorum superiores 4 exigui, convexiusculi et arcte spirati, quintus major ac convexior, tandem penultinus et ullimus relative maximi), irregulariter crescentibus et sutura lineari, inter quintum et penultimum rapide descendente, separatis; ultimo sat amplo, ad initium vix convexiusculo, ad aperturam convexiore; apertura verticali, pyriformi-elongata, superne perangulata, lamellifera (lamella una in ventre penultimi); columella recla, acuminata, intus superne sat ralide denticulata; peristomate obtuso, crasso, patulescente; margine externo parum arcuato; marginibus valido callo junctis; alt. 12 ; diam. 4 , alt. ap. 5 millim.

Anfracluosités de rochers sous l'humus, au nord de Porto-Farina (Berthier).

Sur tes crêtes du Kef (Let.), on rencontre une forme minor à test d'une teinte plus cornèe.

F. eelosia Bourguignat, Malac. Mlg. II, p. 57, pl. IV, f. 14-16, 1864 .

Celle belle Ferussacie, abondante aux environs de Bône, a été retrouvée en Tunisie aux aleutours de Bizerte (Berthier). 
F. ennychia Bourguignat, in Amén. malac. I, p. so^, pl. XtX, f. 10-12, 856 et Malac. Alg. II, p. 58, pl.. IV, f. 17-19, 1864,

Aïn-Draham, vis-à-vis l'ile de Tabarque (Let.).

Fn Algérie, cette espèce est connue de Böne, de Plilippeville et de Constantine.

\section{\$ 10 .}

F. Cossoni Letoumeux of B̧ourguignat, sp, nov.

Testa oroideo-oblonga ac relative tuınida, nitidissima, subpellucida, cornea, polita; spira obesa, ad summum regulariter acuminata, (apex niliilominus obtușiusculus); anfractibus 7 parum convexiusculis, regulariter usque ad quintum leviter convexiorem, dein rapidius crescentibus, sutura lineari, inter ultimos albescente, separatis; ultimo magno, dimidiam altiludinis æquante, mediocriter convexo ; apertura verticali, irregulariter oblonga, intus in ventre penultimi minute lamellifera; columella brevi, arcuata, inferne quasi subtruncatula, superne intus profunde denticulata (denticulus valde remolus, modo oblique perspicuus); peristomate reclo, albescente, incrassalulo; margine externo parum arcuato; marginibus crasso callo junclis; alt. 13 , diam. 5 , alt. ap. 6 1/2 millim.

Djebel Reças et rochers près de Porto-Farina (Let.).

Variété minor au Djebel Zaghouan (Let.).

\section{HOHENWARTHIA (1).}

Cette appellation générique, primitivement établie pour désigner une des coupes sous-génériques dı genre Ferussacia, tel qu'il était compris il y a quelques années, a ćté adoptée par la plupart des malacologistes.

Les Hohenwarthies sont de très petites coquilles vitracées ayant l'apparence de Cacilianelles; elles sont répandues dans toutes les contrées circunméditerranéennes. On en connaitt une cinquantaine d'espèces sur lesquelles six ont été constatées en Algérie (Hoh. mauritanica, eucharista, Paladilhi, Maresiuna, Bourguignatiana et thamnophila) et les trois suivantes en Tunisie.

H. tnnetana Letourneux et Bourguignat, sp. nov.

Testa oblongo-elongata, fragillima, vitracea, nitidissima, hyalina, perpolita; spira producta, ad summum obtusa (apex validus, rolundatus); anfractibus 6 convexiusculis, regulariter ac sat rapide crescentibus, sutura subimpressula separatis; ultimo majore, fere dimidiam altitudinis æquante, convexo ; apertura verticali, oblonga; columella recta, intus in longitudine lamellosa; peristomate recto, fragili; margine externo regulariter arcuato; marginibus tenui callo junctis; alt. 7 , diam. 2 , alt. ap: 3 millim.

Alluvions de l'Oued Sidi-Aich (Let.).

(1) Bourguignat. 1864. 
H. Peehaudi Bourgingnat, sp. nov.

Testa elongata, fragili, "vitracea, nitida, albescente, polita; spira producta, regulariter attenuata, ad summum obtusiuscula (apex exiguus); anfractibus $7-8$ usque ad ultimum vix subconvexiusculis, regulariter ac sat lente crescentibus, sutura lineari separatis; ultimo magno, dimidiam altitudinis fere æquante, sat relative convexiusculo; apertura verticali, irregulariter pyriformi-elongata, superne valde coarctata, intus in ventre penultimi minute lamellata; columella brevi, arcuata, inferne lamellosa ac quasi truncata; peristomate recto, fragili ; "margine externo leviter arcuato; marginibus callo junctis ; alt. 6 1/2, diam. 2 , alt. ap. 3 millim.

Alluvions de la Medjerda près de Ghardimaou (Pechaud).

\section{Hagenmülleri Bourguignat, sp. nov.}

Testa minuta, oblongo-elongata, fragili, diaphana, subalbescente, polita; spira mediocri, producta, ad summum obtusiuscula; anfractibus 6 vix subconvexiusculis, tente usque ad quartum, dein rapidius crescentibus, sutura lineari separatis; ultimo magno, dimidiam altitudinis æquante, convexiusculo; aperlura verticali, pyriformi, superne angustata: columeila brevi, valida, inferne subtruncatula; peristonate recto, fragili; margine externo antrorsum valde arcuato; narginibus sat valido callo junclis; alt. 4, diam. 1 1/2, alt. ap. 2 millim.

Alluvions dé la Medjerda, près de Ghardimaou (Hagenm.).

\section{STENOG IRIDAE.}

\section{RUMTNA ${ }^{(1)}$.}

I. decollata Risso, Hist. Eur. mérid. IV, $1826 ;$ p. -79 (Helix decollata Linnceus, Syst. nat. [ed. X, 1758 ] p. $77^{3}$ et [ed. XII, 1767 ] p. 1247 . Bulimus decollatus, Bruguières, Hist. nal. Vers, in Encycl. meth. I, p. $326,{ }_{1} 7_{9}$ ).

Espèce des plus répandues en Tunisie, ainsi que dans tout le nord de l'Afrique, où on la rencontre partout, particulièrement dans les région's basses êt 'vóisines des côtes.

\section{CAECIUI ANELUID $B$.}

\section{GFCILIANELLA' ${ }^{(2)}$.}

C. raphidia Bourguignat, in Amén. malac. I, p. 218 , pl. XVIII, f. 9-11 et Malac. Alg. II, 1864 , p. 115 , pl. VIII, f. $7-9$.

Très abondante dans les détritus de l'Oued Sidi-Aich (Let.), moins conmune dans les alluvions de la Medjerda, près de Ghardimaou (Pech.).

(2) Risso, 1826 .

(2. Bourguiguat, 1856 . 
En Algérie, celte belle espèce a été trouvée dans les alluvions de la Boudjina, près de Bờne, ainsi que dans celles du Safsaf, près de Philippeville, de l'Harrach, près Alger, de l'Oned Mazafron, près de Sidi-Ferruch, du Cheliff, près Orléansville, etc.; enfin, aux environs de Iostaganem.

c. nanodea Bourguignat, in Amén. malac. I, p. 221 , pI. XVIII, f. 12-14, 1856 et Malac. AIg. II, 1864 , p. 111 , pl. VIII, f. 4-6.

Alluvions de la Medjerda, près le pont de Fondouck (Berthier).

c. Letourneuxi Bourguignat, Nalac. AIg. II, 186 , p. 113 , pI. VIII, f. 1 n-12.

Un seul individu dans les détritus de la Medjerda, près Ghardinıaon (Pechaud). lin Algérie, cette Caccilianelle a été déconverte aux environs d'Algoer, dans les alluvions de l'Harrach et dans celles du ruisseau du Frais-Vallon.

\section{GLA I I N I I AE.}

GLANDINA ${ }^{(1)}$.

G1. dilatata Beck, ind. noll. p. 78,1837 et Bourg. Maluc. Mir. II, 1864, p. 118, pI. VIII, f. 1-3 (Achatina dilatata, Ziegler, mss. Helix rosea, Ferussac in Ilist. moll. pl. CXXXYl, f. 6-7).

Espèce peu commune. Montagne de grè̀ dans la partie haule de la vallée de la Medjerda (Let.).

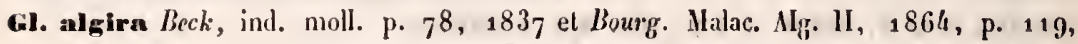
pl. VII, f. 1-12 (Bulimus alyirus Bruguières, Ilisl. nat. Vers, in encycl. meth. p. 364,1792$)$.

Eıvirons de Bizerte (Berthier). Un exemplaire dans les détritus de la Merjerda près Gharlimanu (Pechaud).

A U KICULID E.

\section{CARYCHIUM (2).}

C. minimum Mäller, Verm. Hist. II, p. 195, $177^{4}$.

Alluvions de la Medjerda, près Ghardimaou (Pechaud).

\section{LAIMODONTA ${ }^{(3)}$}

L. Firmini Bourguignat, 1877 (Auricnla Firmini, Pryraudeat, Moll. Corse, p. 105, pl. V, f. 10,1826 ; Nlexia Firmini, Bourg. Malac. AIg., II, p. 143, pI. VIII, f. 40-44, 1864; Pythia Firmini, Beck, 1837 ; Marinula Firmini, L. Pfeiffer, 1856 , $1876)$.

Embouchure de l'Oued Akarit (André); plage dans l'île Djerba, non loiı de Hount-Sonk (Let.).

(1) Schumacker, 1812 .

(2) Müller, $177^{4}$.

(3) H. et A. Adans, 1855. 


\section{ALEXIA (1).}

Les espèces tunisiennes de ce genre sont, à notre connaissance, au nombre de douze. Nous n'avons pas compris dans ce nombre l'Alexia bidentata, signalée de l'embouchure de l'Oued Akarit (André) par M. Morlet (in Arch. miss. sc., p. 395 , 1881), parce qu'il nous semble plus que douteux que la vraie $A$. bidentata, espèce océanienne, puisse se retrouver sur les côtes méridionales de la Tunisie.

\section{$\$ 1$.}

A. enhalia Bourguignat, 1868.

Testa elongata, vitracea, nitidissima, diaphano-luteola, subtiliter striatula aut lævigato-polita; spira producta, longe attenuata (apex acutus, minutissimus); anfractibus 7-8 convexiusculis, regulariter lenteque crescentibus, sutura lineari duplicataque separatis; ultimo magno, dimidiam altitudinis æquante, convexo, ad insertionem breviter deflexo; apertura subobliqua, elongato-pyriformi, intus in pariete unilamellata; columella brevi, recta, profunde unidentata (dens immersus, oblique modo perspicuus); peristomate recto, subobtusato, inferius subpatulescente; margine externo leviter subsinuoso; marginibus callo, super locum perforationis reflexo, junctis; alt. 8 , diam. 3 , alt. ap. 4 millim.

Cette espèce, dont le type a été découvert sur le littoral de Salces, dans les Pyrénées-Orientales, vit en 'Tunisie, à l'embouchure de l'Oued Gabès (Let.).

A. Micheli Bourguignat, Mal. Alg. Il, p. 140 , pl. VIII, f. $34-39,1864$ (Auricula Micheli, Mittre in Rev. zool. p. 66, 1841 ; Leuconia Micheli, H. et A. Adams, 1854).

Bords de l'Oued Akarit (André); lagune de l'Oued Cheiba au Cap Bon et environs de Porto-Farina (Let.).

Le professeur Issel (Croc. del Violante, Moll., p. 104, 1880) a signalé la variété triplicata (Bourg. Mal. Alg. II, p. 141, pl. VIII, f. 37 ) dans l'âle Galite et même de Tozer, au nord du Chott El-Djeria (Kerim, ${ }^{8} 7_{76}$ ).

A. myosotis Mörch, Cat. Yoldi, p. 38, 1852 et Bourg. Malac. Alg. II, p. 138, pl. VIII, f. 20-2 2, 1864 (Auricula myosotis, Draparnaud, Tabl. Moll. p. 53, 1801 et Hist. Moll. Fr. p. 56, pl. III, f. 16-1 7, 1805).

Littoral près Porto-Farina; lagune de l'Oued Cheiba au Cap Bon et plage de Gabès (Let.); bords du lac Bahirah entre Tunis et la Goulette (Pechaud).

\section{S2.}

A. algerica Bourguignat, Malac. Alg. II, p. 141, pl. VIII, f.. $23-30,1864$.

Espèce assez abondante sur le littoral et même dans quelques cours d'eau de l'intérieur des terres.

(i) Leach, 1818 . 
Oasis de Kebilli (Bellucci, $188_{7} 5$ ); Aïn-Oudref, Oued Akarit, Oued Melah et Keriz, au nord du Chott El-Djerid (André), lagune de l'Oued Cheiba au Cap Bon; embouchure de l'Oued Gabès, enfin ruisseau à El-Hamma, au sud de Gabès (Let.).

A. baleariea Dohrn et Heynemann, Moll. Balear. in Malak. Blätter, p. 110, 1862.

Cetle jolie Alexie espagnole semble assez répandue sur la plage de Gabès, ainsi qu'à l'embouchure de l'Oued Gabès; on la rencontre encore, mais plus rarement, à El-Hanma, au sud de Gabès, dans l'intérieur des terres (Let.).

A. Cossoni Letoumeux et Bourguignat, sp. nov.

Testa curta, ventrosa, ovoidea, nitida, translucida, pallide luteola, ad summum rubella et ad aperturam albo-marginata, subtiliter striatula; spira breviter acuminata (apex peracutus); anfractibus 7 convexiusculis, arcte spiratis, sutura impressula separatis; ultimo magno, dimidiam altitudinis superante, rotundato, ventroso, nihilominus inferne altenuato; apertura subobliqua, elongata sat angusta, intus quadridentata, scilicet: dentes duo in pariete aperturali quorum superior minimus ac sæpe deficiens; columellaris unus validus prominens ac contortus, tandem (modo in speciminibus grandæris) denticulus unus intus ad partem superam marginis exterui; columella brevi, robusta; peristomate recto, acuto, intus (apud specimina adultissima) albo-incrassato, inferne patulescente; margine externo recte descendente; marginibus callo diaphano, super locum perforationis sat late reflexo, junctis; alt. 7 , diam. 4 , alt. ap. 4 millim.

Cette Alexie, remarquable par sa forme écourtée, par sa coquille très ventrue, par sa spire brièvement acuminée, par sa lamelle columellaire contournée et faisant saillie sur la face de l'axe, etc., a été recueillie à l'embouchure de l'Oued Gabès, ainsi que dans la lagune de l'Oued Cheiba au Cap Bon (Jet.).

A. dubia $L$. Pfeiffer, Syn. Auricul. $\mathrm{n}^{\circ} 157,1854$, et Monogr. Auricul. viv., p. 150 , 1856 (Auricula dubia, Cantraine, in Bull. Soc. sc. Brux. II, p. 383, 1836).

Embouchure de lOned Gabès (Let.).

A. terrestris Letouneux et Bourguignat, sp. nov.

Testa parva, ventrosa, curta, utrinque attenuata, luteolo-albescente, ad summum rubella, fere lævigata; spira breviter conica (apex acutus); anfractibus 6 vix convexiusculis, arcte spiratis, sutura lineari separatis ; ultimo relative maximo, dimidiam altitudinis superante, turgido-ventroso, ad basin attenuato, ad insertionem breviter deflexo; apertura vix subobliqua, angustata, elongata, superne perangulata, inferne leviter coarctata, intus bilamellata (lamella una lamellifornis in pariete aperturali; altera columellaris contorta, valde prominens ac fere usque ad basin des- 
cendens); columella brevissima, recta; peristomate recto, acuto, inferne intus incrassato ac patente; margine externo antrorsum regulariter subarcuato ; marginibus callo, super locum perforationis late reflexo, junctis; alt. 5 , diam. 3 , alt. ap. 3 millim.

Cette petite espèce se rencontre dans l'intérieur des terres, sur le bord d'un ruisselet, à El-Hamma, au sud de Gabès (Let.).

\section{A. globulus Bourguignat, sp. nov.}

Testa parvula, curta, ventrosa, globulosa, nitida, diaphana, cornea, prope aperturam albescente, subtiliter striatula; spira valde curta, obesa nihilominus conica (apex prominens ac quasi mamillatus); anfractibus 6 convexiusculis (convexitas maxima ad suturam proxima), arcte spiratis usque ad penultimum, dein rapidius crescentibus, sutura impressula separatis; ultimo maximo, $2 / 3$ altitudinis æquante, superne turgido, inferne attenuato; apertura subobliqua, angusta, oblongo-elongata, trilamellata (parietales duæ quarum superior minuta ac denticuliformis, inferior lamelliformis; columellaris una contorta, sat immersa); columella brevi, alba; peristomate recto, albo, inferne patente ac intus albo-incrassato; margine externo antrorsum non arcuato sed recto; marginibus callo diaphano vix perspicuo junctis; alt. 6 , diam. $31 / 2$, alt. ap. 4 millim.

Petit canal d'irrigation dans l'intérieur de l'oasis de Gabès (Duveyrier).

\section{S 3 .}

A. Bivonse Bourguignat, Malac. Alg. II, p. 137, 1864 (Ovatella bidentata (1), Ant. Bivona, Nuov. Gener. p. 22, pl. II, fig. 10, 1832 ; Auricula myosotis, var. B, Philippi, Enum. Moll. Sic. I, p. 143, 1836; Auricula Bivonæ, Philippi [loc. sup. cit.] II, p. 118, 1844).

Plage de Gabès et rochers à fleur d'eau près de Porto-Farina (Let.).

\section{A. Letournenxi Bourguignat, sp. nov.}

Testa sat parvula, oblonga, mediocriter ventrosa, nitida, sat fragili et translucida, cornea cum flammulis rubellis ac fere lævigata; spira regulariter attenuata, sat producta (apex acutus, prominens); anfractibus 6-7 convexiusculis, regulariter crescentibus, sutura impressula separatis; ultimo magno, dimidiam altitudinis leviter superante, convexo; apertura obliqua, angustata, elongata, eleganter albo-multidentata, scilicet: dentes parietales 3 lamelliformes quorum superior minimus et inferior validior; columellaris unus, robustus, lamelliformis, contortus ac fere usque ad basin descendens; tandem palatales 5 dentiformes, intus in margine

(1) Non Montagu. 
externo ac extus apparentes; columella robusta, brevissima; peristomate recto, breviter patulescente; margine externo recto; marginibus tenui callo junctis; alt. $51 / 2$, diam. $21 / 2$, alt. ap. 3 millim.

Chez cette belle espèce, les cinq dents palatales se poursuivent dans l'intérieur et donnent lieu à trois ou quatre séries de denticulations analogues à celles qui caractérisent l'ouverture.

Cette Alexie, qui vit en abondance sur les rochers de Mandara, près d'Alexandrie, en Égypte, a été retrouvée sur le littoral de l'île Djerba (Let.).

\section{A. Pechaudi Bourguignat, sp. nov.}

Testa parvula, breviter fusiformi, utrinque attenuata, in medio relative lurgida, nitida, levigata, cornea ; spira conica (apex prominens et acutus); anfractibus 6 convexiusculis, arcte spiratis, sutura impressula separatis; ultimo magno, dimidiam altitudinis æquante, superne turgido, inferne attenuato; apertura subobliqua, angustato-elongata, albo-quinquedentata : dentes parietales 3 quorum superiores 2 minuti, inter se sat approximati ac ad partem superam notati, inferior pervalidus, lamelliformis prope columellam; columellaris unus robustus, contortus; tandem palatalis unicus dentiformis in margine externo; columella brevi, crassa; peristomate recto, albo-incrassatulo, inferne leviter patulescente; margine externo antrorsum vix arcuatulo; marginibus valido candidoque callo junctis; alt. 5 , diam. 2 1/2, alt. ap. $21 / 2$ millim.

Cette espèce, découverte dans la Macta, près d'Oran, par M. Pechaud, a été recueillie, en Tunisie, par ce malacologiste dans les alluvions de la Medjerda, près de son embouchure, dans les marais d'Utique.

\section{\$2. PULMOBRANCHIATA.}

\section{A NCY LID}

\section{ANCYLUS.}

A. strigatus Parreyss, in Bourguignat, in Journ. conch. IV, p. $19^{3}, 1853$ et in Spicit. malac. p. 166,1862 .

Espèce abondante dans une source, aı pied de la colline de Takrouna (domaine de l'Enfida), ainsi que dans les ruisseaux des forêts des Merasen et des Oulad-Ali (Let.), enfin dans l'Oued El-Hadjar, chez les Ouchteta (Roger Marès).

Cet Ancyle est une espèce sicilienne qui, dans ces derniers temps, a été constatée en Italie et en Espagne.

A. Peraudieri Bourguignat, in Spicil. malac. p. 197, 1862, et Malac. Alg. II, p. 204. pl. XIII, f. 6-9, 1864 .

Ruisseau de l'Aïn-Medeker, dans l'Enfida (Let.), où cette espèce algérienne est commune. 


\section{LIMN $A$ ID $A E$.}

\section{IIMINAEA.}

L. Vatonni Bourguignat, Malac. Tunis, p. 31, pl. I, f. 32-33, 1868.

Sources thermales du Djerid (Duveyrier). Le type a été recueilli dans l'oasis de Ghadamès (Vatonne).

L. truncatula Goupil, Moll. Sarthe, p. 64, pl. II, f. 1-3, 1843 (Buccinum truncatulum, Müller, Verm. Hist. II, p. 130, 1774; Limnæus truncatulus, Jeffreyss, 1830 ; Limnæus minutus, Draparnaud, 1801 et 1805 ; Limnæa minuta, Lamarck, 1822 ).

Cette espèce cosmopolite a été constatée en Tunisie dans un petit ruisseau, sur le rivage méridional de la Galite (Gestro, 1877), dans les alluvions de la Medjerda, près du pont de Fondouk (Bourg. 1867) et près de Ghardimaou (Pech.), ainsi que dans les ruisseaux du Djebel Zaghouan (Let.), des environs de Gafsa (Doûm.-Ad.), enfin dans l'Aïn-Zerig et les fossés de l'oasis de Gabès (Let.).

\section{PHYSA.}

Ph. Brondeli Bourguignat, in Amén. malac. I, p. 173, pl. XXI, f. 11-13, 1856 et Malac. Alg. II, p. 177 , pl. X, f. 43-44, 1864 .

Alluvions du lac Kelbia (Let.).

Le type de cette espèce vit aux environs de Bône.

Ph. truncata Ferussac, mss. in Bourg. in Amén. malac. I, p. 170, pl. XXI, f. 5-7, 1856 et Malac. Alg. II, p. 176 , pl. X, f. $47-49,1864$.

Lac Kelbia (Let.), Gafsa (Doûm.-Ad.).

Ph. Brocchii Bourguignat, in Amén. malac. I, p. 169,1856 et Malac. Alg. Il, p. 174 , pl. X, f. 45-46, 1864 (Isidora Brocchii, Ehrenberg, Symb. phys. Moll. 1831).

Alluvions de la Medjerda, au-dessous du pont de Fondouk (Bourg. 1867); environs de Gafsa (Doûm.-Ad.); bords du lac Kelbia (Let.), où cette espèce offre de grandes variations de taille.

Ph. Raymondiana Bourguignat, in Amén. malac. I, p. 172, pl. XXI, f. 8-1 o, 1856 et Malac. Alg. II, p. 173, pl. X, f. 41-42, 1864.

Lac Kelbia (Let.). Échantillons bien typiques.

Ph. contorta Michaud in Act. Soc. linn. Bordeaux, III, p. 268,1829 et Cat. test. viv. Alg. p. 12 , f. ${ }_{26-27}, 1833$ et Bourg. Malac. Alg. II, p. 171, pl. X, f. 38-40, 1864.

Également dans Je lac Kelbia (Let.). 


\section{PUA NOR BIDAE.}

\section{PLANORBIS ${ }^{(1)}$}

PI. marmoratus Michaud, Cat. test. viv. Alger, p. II, f. 28-30, 1833 (Planorbis complanatus, var. C. Bourg. Malac. Alg. II, p. 153 , pl. IX, f. $23-26,1864$ ).

Oned Gabès (Doûm.-Ad.).

PI. submarginatus Cristofori et Jan, Mant. $\mathrm{n}^{\circ}$ 9, 183 a (Planorbis complanatus var. B. Bourg. Malac. Alg. II, p. 152, pl. IX, f. 20-32, 1864 ).

Fossés et cours d'eau de l'oasis de Gabès (Let.).

PI. subangulatus Philippi, Moll. Sicilix, II, p. 119 , pl. XXI, f. 6, 1844 et Bourg. Malac. AIg. II, p. 153, pl. IX, f. 27-30, 1864.

Avec l'espèce précédente, dans l'oasis de Gabès (Let.), ainsi que dans l'Oued Melah (André).

Pl. agraulus Bourguignat, Malac. Alg. II, p. 159 , pl. X, f. 22-25, 1864 .

Bords du lae Kelbia (Let.).

PI. numldicus Bourguignat, Malac. Alg. II, p. 160 , pl. X, f. ${ }_{96-29}, 1864$.

Petits ruisseanx, chez les Ouchteta (Let.).

\section{GASTEROPODA OPERCULATA.}

\section{PULMONAGEA. \\ CYCIOSTOMIDAE. \\ CYCLOSTOMA ${ }^{(2)}$.}

C. suleatum Draparnaud, Hist. Moll., p. 33, pl. XIII, f. 1, 1805.

Sur les rocliers, aux environs de Porto-Farina (Let.), et de Tunis (Bourg., 1867 ).

\section{POMATIAS ${ }^{(3)}$.}

Les Pomatias aetuellement connus dans le nord de l'Afrique, en y comprenant les espèces tunisiennes que nous allons déerire, sont au nombre de dix-neuf.

(I) Guettard, 1756.

(2) Draparnaud, 1801.

(3) Studer, 1789 . 
Ces Pomatias peuvent se répartir en cinq groupes :

$1^{\circ}$ Pomatias oranicus (Bourg. 1875 ).

Cette espèce appartient à la série du Pomatias obscurus des Pyrénées;

$2^{\circ}$ Pomatias Belloiri (Let.) et Latasteanus (Let. et Bourg.);

$3^{\circ}$ Pomatias Letourneuxi (Bourg.), tunetanus (Let. et Bourg.), Henoni (Bourg.), euristoma (Bourg.), funereus (Bourg.), Rokniacus (Bourg.), cyclonixius (Bourg.), Rogeri (Let. et Bourg.) et euneus (Bourg.);

$4^{\circ}$ Pomatias monticola (Bourg.), Thayacus (Bourg.), Doumeti (Let. et Bourg.) et punicus (Let. et Bourg.);

Enfin, $5^{\circ}$ Pomatias Maresi (Let.), Pechaudi (Bourg.) et atlanticus (Let.).

Les espèces de ce dernier groupe paraissent spéciales aux montagnes de la Kabylie.

$\$ 1$.

\section{P. Belloiri Letourneux, sp. nov.}

Testa punctiformi-perforata (perforatio minutissima, sæpe semitecta), turrito-conica, nihilominus in medio leviter tumidula, fragili, subtranslucida, parum nitente, undique (embryonalibus lævigatis exceptis) confertim costulata (costæ regulares, sat productæ), albo-cærulescente, ad summum fuscula aut cornea et in ultimis biseriatim fusco-maculata; spira conica, ad summum obtusa (apex mamillatus, lævis ac nitidus); anfractibus $9^{-1} 0$ relative sat convexis, lente crescentibus, sutura bene impressa separatis; ultimo mediocri, rotundato, ad aperturam campanulatim vix ampliore; apertura verticali, sphærica; peristomate fragili, undique leviter patulescente, ad marginem columellarem vix auriculato; alt. 13 , diam. 5 , alt. ap. $31 / 2$ millim.

Celte espèce, découverte sur les rochers des Djebel Thaya et Guerar, dans la province de Constantine, a été retrouvée en Tunisie, non loin de la source du Djebel Zaghouan.

\section{P. Latasteanus Letourneux et Bourguignat, sp. nov.}

Testa punctiformi-perforata, turrito-conica, in medio tumidula, subopacula, minus fragili, leviter nitidula, confertim (embryonalibus exceptis) costulata, uniformiter pallide flavo-albescente; spira conica, ad summum obtuso-mamillata (apex amplus, lævis, nitidissimus); anfractibus 9 convexis, lente crescentibus, sutura profunda separatis; ultimo rotundato, inferne obscure subangulato, ad aperturam campanulatim dilatato ac superne ad insertionem breviter ascendente; apertura subverticali, rotundata, superne obsolete angulata, intus incrassata; peristomate simplici, intus crassulo, undique patulo, ad marginem columellarem auriculato; operculo (ignoto); alt. 10, diam. 4, alt. ap. 3 millim. 
Le $P$. Latasteanus se distingue du précédent par sa taille moindre, par son test plus solide, par sa coloration différente, sans macules, par ses tours plus convexes, par sa suture profonde, par son dernier tour très dilaté aux abords du péristome, par son ouverture arrondie avec un sentiment d'angulosité relativement prononcé à son bord externe supérieur, par son péristome intérieurement épaissi et largement épanoui en forme d'entonnoir.

Sur les rochers, au Djebel Reças (Let.).

\section{S 2.}

P. Letourneuxi Bourguignat, Moll. nouv. ( $7^{\circ}$ décade, 1866 ), p. 216 , pl. XXXIII, f. 22-23, et Saint-Simon, Desc. esp. nouv. Pomat. p. 17, 1869, et L. Pfeiffer, Mon. preamonop. (supplém. III), p. 199,1876 .

Primitivement découverte à Roknia, près Haminam-Mesklıoutin, où elle se trouve en grande abondance, cetle espèce a été fréquemment constatée en Tunisie, au Djebel Reças (Bell., 1875 et Let.), au Djebel Bou-Kourneïn (Doûm.-Ad.), ainsi qu'au Djebel Zaghouan, à Mokta-el-Hadjar, à Hammam-Zeriba, dans l'Enfida, et au Kef El-Hannech, chez les Oulad-Ali (Let.).

\section{P. tunetanus Letourneux et Bourguignat, sp. nov.}

Testa punctiformi-perforata, conica, opacula, nitidula, eleganter (embryonalibus exceptis) costulata, uniformiter pallide pruneo-cinerescente; spira regulariter conico-attenuata, ad summum leviter mamillata (apex lævis, pallide luteus, nitidissimus); anfractibus 9 vix convexiusculis, sutura mediocriter impressa separatis; ultimo rotundato, ad aperturam campanulatim amplo, superne ad insertionem breviter ascendente; apertura subverticali, superne obscure angulata, rotundata, nihilominus plerumque ad marginem externum leviter compressa, intus fuscula, ad marginem albo-nitida; peristomate simplici aut raro in speciminibus grandævis leviter duplici, candido, acuto, intus albolabiato, ad marginem externum campanulatim expanso, tandem ad insertionem et super marginem columellarem biauriculato; operculo (ignoto); alt. 10 , diam. 4, alt. ap. $31 / 4$ millim.

Ce Pomatias, remarquable par sa coloration, non moins que par son ouverture comprimée du còté externe, semble assez répandu sur les montagnes, au-dessus d'Hammam-el-Lif, au Zaghouan, sur les collines près de l'Oued El-Hammam, ainsi que dans le domaine de l'Enfida, à Takrouna et à Hammam-Zeriba (Let.).

P. Wenoni Bourguignat in Saint-Simon, Desc. esp. nouv. Pomat., p. 21 (sans description), 1869 .

Testa imperforata aut punctiformi-perforata, turrito-acuminata, opacula, non nitente, cinerescente aut pallide luteola, valide (embryonalibus exceptis) costulata; spira regulariter acuminata, ad summum obtusa (apex lævis, luteus, splendens); anfractibus 9 leviter convexiusculis, 
sutura sat bene impressa separatis; ultimo rotundato, ad aperturam campanulatim et præcipue ad basin ampliato; apertura subobliqua, perviorotundata, superne ad insertionem obscure subangulata, intus sæpe plus minusve castanea; peristomate simplici, non continuo (margines tenui callo juncti), acuto, expanso ac in campanulam undique dilatato et biauriculato (auricula superior ad insertionem labri fere omnino adnata ac relative ascendens; auricula inferior obsoleta; a penultimo spatio profunde canaliculato late separata): operculo (ignoto); alt. 12, diam. 5, alt. ap. 4 millim.

la large dilatation en entonnoir de l'ouverture, non moins que la profonde échancrure de l'axe columellaire à sa partie supérieure permettront toujours de distinguer aisément cette espèce des précédentes.

Ce Pomatias, d'abord découvert à El-Harrouch, entre Philippeville et Constantine, puis à Roknia, près Hammam-Meskhoutin, a été retrouvé en Tunisie, dans les anfractuosités des rochers, au Djebel Bou-Kourneïn (Doûm.-Ad.), ainsi qu'au Djebel Reças, aux environs d'Hammam-el-Lif, et aux alentours de Mokta-elHadjar (Let.).

P. euristoma Bourguignat, sp. nov.

Testa obtecte perforata, sat breviter conico-acuminata, opacula, non nitente, sæpe limo inquinata, uniformiter pallide albescente aut potius fuscula, valide (embryonalibus exceptis) costata (costæ robustæ, productæ, distantes); spira conica, ad summum obtuso-mamillata (apex lævis ac micans); anfractibus 9 convexiusculis, sutura sat profunda separatis ; ultimo rotundato, inferne obscure subangulato, ad aperturam ampliato; apertura verticali, exacte rotundata, nihilominus ad insertionem obscure subangulata; peristomate continuo, simplici aut in speciminibus grandævis duplici, valde incrassato, late plane expanso ac biauriculato (auricula superior omnino adnata; auricula inferior ampla, alata, convexitatem penultimi fere attingens); operculo (ignoto); alt. 10 , diam. 5, alt. ap. 4 millim.

Cette espèce, remarquable par ses fortes côtes, par sa belle oreillette columellaire ailée, par la grande expansion de son péristome, d'abord découverte à Roknia, puis à Hammam-Meskhoutin, a été retrouvée en Tunisie, au Kef El-Hanneck, chez les Oulad-Ali (Let.).

\section{P. cyclonixius Bourguignat, sp. nov.}

Testa punctiformi-perforata, conica, opacula, non nitente, sat tenuiter costulata, pallide fuscula ac obscure fasciata (fasciæ duæ [in ultimo tres] subcastaneæ ac interruptæ); spira acuminata, ad summum obtusa (apex lævis et splendens); anfractibus 9 convexiusculis, sutura sat profunda separatis; ultimo mediocri, rotundato, inferne obsolete subangulato ac 
infra leviter subplanulato; apertura vix obliqua, exacte sphærica; peristomate albescente, non continuo (margines callo juncti), mediocriter crasso, breviter reflexo ac ad marginem columellarem auriculato (auricula obsoleta, a convexitate penultimi valde remota); operculo (ignoto); alt. 10 , diam. 4, alt. ap. $21 / 2$ millim.

Cette belle espèce, dont le type provient de Roknia, dans la province de Constantine, a été recueillie dans le Djebel Zaghrouan (Let.), où elle parait fort rare.

\section{P. Rogeri Letourneux et Bourguignat, sp. nov.}

Testa subobtecte perforata, turrito-acuminata, relative elongata, subopacula, non nitente, cinerescente, eleganter costulata; spira producta, ad summum obtusa (apex lævis, luteolus ac nitidus); anfractibus 10 convexiusculis, sat arcte spiratis, sutura impressa separatis; ultimo rotundato, inferne obscure subangulato, ad aperturam campanulatim ampliato; apertura verticali, ad dextram valde projecta ac quasi excentrica, rotundata, superne angulata ; peristomate non continuo (margines callo juncti), incrassatulo, inferne late planeque expanso, ad insertionem antrorsum reflexiusculo, ad columellam valde auriculato (auricula ampla, superne abrupte desecta ac convexitati penultimi proxima); operculo (ignoto); alt. 11 1/2, diam. 5, alt. ap. 4 millim.

Cetle singulière espèce, caractérisée par son ouverture excentrique, vit au Kef El-Hanneck, chez les Oulad-Ali, où clle a été trouvée par M. Roger Marès, à qui elle est dédiée.

\section{P. euneus Bourguignat, sp. nov.}

Testa subpunctiformi-perforata, relative minuta, conica, parum nitente, eleganter costulata, cinerescente ac fusco-bifasciata (fasciæ interruptæ); spira breviter acuminata, ad summum obtusa (apex lavis, nitidus); anfractibus $\delta$ sat convexiusculis, sutura relative profunda separatis; ultimo rotundato, ad aperturam ampliato; apertura verticali, rotundata, superne obscure subangulata; peristomate non continuo (margines callo juncti), simplici, acuto, undique mediocriter expanso ac biauriculato (auricula superior subadnata; auricula inferior plus minusve ampla aut obsoleta, a convexitate penultimi sat remota); operculo (ignoto); alt. 8, diam. 4, alt. ap. 3 millim.

Le type de cette petite espèce vit sur les rochers des Djebel Guerar et Thaya, dans la province de Constantine.

En Tunisie, ce Pomatias a été constaté au Djebel Reças et sur les collines au dessus d'Hammam-el-Lif (Let.). 


\section{S 3.}

Les espèces de ce groupe sont caractérisées par une croissance plus lente et par un péristome épais, droit ou à peine évasé, possédant un double rebord émoussé, l'interne dépassant l'externe.

P. monticola Bourguignat, sp. nov.

Testa punctiformi-perforata, turrito-conica, opaca, non nitente, sordide fusca, eleganter costata (costæ productæ ac robustæ); spira sat elongata, acuminata, ad summum obtusa (apex lævis, micans ac luteolus); anfractibus 9 convexiusculis, lente crescentibus, sutura sat profunda separatis; ultimo exacte rotundato, ad aperturam vix ampliore; apertura verticali, fere sphærica, ad insertionem subangulata, intus albescente ac incrassatula; peristomate crasso, obtuso, duplici (internum continuum, eminens; externum ad columellam obsolete auriculatum); operculo (ignoto); alt. 10 , diam. $41 / 2$, alt. ap. $23 / 4$ millim.

Le bord péristomal de cette espèce ressemble d'une façon frappante à celui qui caractérise la Stalioa prototypica des marnes tertiaires de la Dalmatie.

Le type de ce Pomatias se trouve sur le Djebel Guerar, haute montagne près d'Hammam-Meskhoutin, dans la province de Constantine.

En Tunisie, cette même espèce a été constatée sur les sommets des Djebel Reças et Bou-Kourneïn (Let.).

\section{P. Donmeti Letourneux et Bourguignat, sp. nov.}

Testa punctiformi-perforata, turrito-conica, opaca, non nitente, pallide subcarneolo-albescente, tenuiter costulata (costulæ albidæ, parum validæ, in superioribus confertæ, in ultimis distantes, ad aperturam evanidæ); spira regulariter conico-attenuata, ad summum obtusa (apex validus, lævis, splendens et nitidus); anfractibus 9 convexiusculis, arcte spiratis, sutura impressula separatis; ultimo rotundato, inferne circa perforationem leviter subplanulato, ad aperturam mediocriter ampliato; apertura subobliqua, rotundata in directionem leviter declivem a sinistra ad dextram ac quasi subexcentrica, superne subangulata, intus incrassata ac albescente; peristomate crasso, obtuso, duplici (internum continuum; externum valde diminutum, inauriculatum); operculo (ignoto); alt. 12, diam. 5 , alt. ap. 4 millim.

Anfractuosités des rocbers, au Djebel Bou-Kourneïn (Let.).

\section{P. punicus Letourneux et Bourguignat, sp. nov.}

Testa punctiformi-perforata, relative minuta ac brevi, conoidali, opaca, corneo-fusca, valide (embryonalibus exceptis) costulata ; spirabreviter conica, ad summum obtusiuscula (apex lævis, corneus et nitidus); anfractibus 8 convexiusculis, arcte spiratis, sutura impressula separatis ; 
ultimo rotundato, ad aperturam valde ampliato; apertura verticali, rotundato-pyriformi, superne angulata, intus incrassata ac albescente; peristomate crasso, obtuso, duplici (internum continuum, prominens; externum sordidum ac ad insertionem labri et ad columellam leviter auriculatum); operculo (ignoto); alt. 9 , diam. 4 1/2, alt. ap. 3 millim.

Çà et là, au Djebel Reças (Let.).

\section{ACME ${ }^{(1)}$.}

A. Letourneuxi Bourguignat, Malac. Alg. II, p. 221, pI. XIII, f. $31-33,1864$.

Alluvions de l'Oued Akarit (André).

A. Benoiti Bourguignat, Malac. Alg. II, p. 218,1864 , et Paladilhe in Nouv. miscell. malac. ( $3^{\circ}$ fasc. 1868), p. $9^{3}$ (Pupula lineata, Calcara, 1844; Acme lineata, Benoit, 1857).

Cetle espèce sicilienne, constatée dans ces derniers temps aux environs de Bòne, vit également en Tunisie, où elle a été recueillie dans les alluvions de la Medjerda, au-dessous de Ghardimaou (Pech.).

\section{ASSIMIN1D}

\section{ASSIMIINLA (2).}

As. Littorina Paladilhe, Desc.quelq. nouv. esp. et Prodr. monogr. Assimin. d'Europe (ext. E. Ann. sc. nat. Paris, 1875 ), p. 10,1875 (Helix littorina, Delle Chiaje, Mem. Anim. s. vert. Napoli, III, p. 215 , pl. XLIX, f. $36-38,1828$ ).

Sur les pierres mouillées par l'eau de la mer, près de Porto-Farina (Let.). Abondante.

Cette espèce, répandue sur les côtes de la Sicile, de l'Italie, des Baléares, de Crète, etc., a été constatée même en Égypte, à Mandara, près Alexandrie, où elle vit en compagnie de l'Assiminia Letourneuxi (Bourg.).

\section{§. BRANCHIATA.}

\section{PA HUID I I I $A$.}

\section{BYTHINLA ${ }^{(3)}$.}

Les espèces de ce genre, dans nos possessions du nord de l'Afrique, sont au nombre de huit, savoir :

Bythinia tentaculata, Gray, Turt. Man. 2édit., p. 93, 1840 (Helix tentacu-

(1) Hartmann, 1821.

(ข) Leach, 1816 .

(ग) Gray, 1821 . 
lata, Limnoeus, 1758 ; Paludina impura, Brard, 1815), de Chabet-Beinan, près Alger;

B. numidica Bourguignat, Malac. Alg. II, p. 225, pl. XIII, f. 42-44, 1844 , des environs de Bône;

B. Bourlieri, Letourneux, sp. nov. 188o, d'Ouargla;

B. Orsinii, Charpentier, mss. (Paludina Orsinii; Kuster, Palud. p. 42, pl. IX, f. 1-2, 1852), du lac des Arbaouam, dans l'Edough et des Seba;

B. Hipponensis, Bourguignat, sp. nov. 1881, des environs de Bône;

B. Sebanica, Bourguignat, sp. nov. 1881 , des Seba;

B. Hagenmülleri, Bourguiggnat, sp. nov. 1881, des alentours de Bône,

Et B. punica, Hagenmüller, sp. nov. 1881, également des environs de Bône.

Ces deux dernières espèces sont les seules qui aient été jusqu'à présent rencontrées en Tunisie.

\section{B. Hagenmälleri Bourguignat, sp. nov.}

Testa parvula, vix rimata, pertumida, subopaca, fere lævigata, nitida ac cornea; apice valido et obtuso; anfractibus 4 perventrosis, exacte cylindricis, regulariter crescentibus, sutura valde profunda separatis; ultimo mediocriter majore, dimidiam altitudinis æquante, perventroso ac exacte rotundato; apertura fere verticali, ovata; peristomate continuo, recto, nigrescente; operculo lineolis concentricis prominentibus insigni; alt. $31 / 2$, diam. $21 / 2$, alt. ap. $13 / 4$ millim.

Cette espèce, du groupe de la $B$. Orsinii, a été recueillie dans un petit lac, au sud de Bizerte (Berthier).

Le type de cette Bythinie vit dans le lac de Faid El-Maïz, près de Bỏne, où il a élé découvert par le $\mathrm{D}^{\mathrm{r}}$ Hagenmüller.

\section{B. punica Hagenmüller, sp. nov. 1881 .}

Testa sat parvula, rimata, tumido-ovata, opacula, lævigata, parum nitida, cornea aut sæpe limo rubro inquinata; apice minuto; anfractibus 5 ventrosis, sat celeriter crescentibus, sutura mediocriter profunda separatis; ultimo rotundato, dimidiam altitudinis æquante; apertura verticali, ovata; peristomate continuo, recto, leviter subpatuło, atro aut albescente; operculo lineolis concentricis tenellis cincto; alt. 4 1/2, diam. 3; alt. ap. 2 1/4 millim.

Cette espèce, dont le type a été également découvert faux environs de Bône, a été recueillie dans les alluvions des lacs de Bizerte (Berthier).

Cetle Bythinie se distingue de la précédente par sa taille plus forte, par sa croissance spirale un peu plus rapide, par son sommet exigu, mais notamment par ses tours plus nombreux, moins renflés et séparés par une suture relativement peu profonde. Chez la $B$. Hagenmülleri, la suture est si enfoncée que les tours, qui sont bien renflés-cylindriques, paraissent comme détachés. 


\section{AMNTCOLA ${ }^{(1)}$.}

Les Amnicola, du nord de l'Afrique, peuvent se répartir dans les huil séries suivantes :

$1^{\circ}$ Les anormales ${ }^{(2)}$ (type $A$. singularis), parmi lesquelles il convient de comprendre les A. Oudrefica et paradoxa;

$2^{\circ}$ Les conlques (type $A$. Latasteana), représentées en Afrique par une seule espèce (le type) ressemblant comme forme à l'Helix Bellucciana, décrite à la page 103 ;

$3^{\circ}$ Les oblongoes (type A. cyrniacu), telles que les A. subscalaris, Bythinopsis, Jusi, Cossoni, saharica, Miloni, etc., auxquelles il faut joindre l'A. Monjoi, des Baléares, les $A$. lanceolata, Vindilica, de France, etc.;

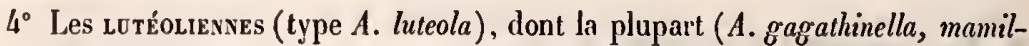
lata, Thessalonica, etc.) sont, à l'exception de l'A. luteola, étrangères à l'Afrique;

$5^{\circ}$ Les simlojexnes (type $A$. sinilis), dont deux seulement $(A$. similis et $M a-$ ceana) ont été constatées dans nos possessions du nord de l'Afrique;

$6^{\circ}$ Les ÉParsses (type A. Pomariensis), telles que les A. pycnocheilia, Pomariensis, desquelles il faut rapprocher les formes étrangères: A. Melitensis, compacta, etc.;

$7^{\circ}$ Les avitines (type $A$. anatina), qui forment un groupe fort nombreux ( $A$. Dupotetiana, Scrvainiana, Rouvieriana, acrambleia, Constantine, etc., d'Afrique, et A. Cocchii, subproducta, Sahara, Isterica, Penchinati, Salinesi, balearica, Emiliana, etc., d'Europe ou des iles méditerranéennes);

Enfin, $8^{\circ}$ les coortes-globuleuses (type $A$. globulina), parmi lesquelles nous comprenons les A. sterea, globulina, Doumeti, pycnolena, Letourneuxiana, perforata, Barattei, ragia, fontium, Gestroi, etc.

\section{1.}

\section{A. singularis Letourneux et Bourguignat, sp. nov.}

Testa punctiformi-perforata, subelongato-oblonga, fere opaca, sublævigata, atra, sine incola corneo-viridescente aut subsmaragdina; spira producta, ad summum sat breviter attenuata; apice exiguo; anfractibus 6 irregulariter crescentibus quorum superiores tres exigui, medianus gibbosus et cæteri ampli, inflati ac circa suturam profundam tumiduli; ultimo relative mediocri, $1 / 3$ altitudinis æquante, rotundato superne

(1) Haldemann, 1841.

(2) Coquille oblongue-allongée, à tours supérieurs très exigus, au tour médian prenant subitement un grand développement en taille et en grosseur, et au dernier tour exactement renflécylindrique. Suture toujours très profonde. 
prope suturam quasi subscalari; apertura leviter obliqua, ovata; peristomate continuo, recto, intus subincrassatulo ac subpatulo; operculo atro, pervelociter spirescente; alt. 6 , diam. 4, alt. ap. 2 , lat. ap. $13 / 4$ millim.

Cette espèce, remarquable par sa croissance spirale subscalariforme et par l'énorme développement en taille et en grosseur de ses derniers tours, vit dans l'Oued Gabès, près de Gabès, ainsi que dans les ruisseaux de l'oasis d'Oudref (Let.).

\section{A. Oudrefica Letourneux et Bourguignat, sp. nov.}

Testa rimato-perforata, subconoidea, inferne inflata, superne obtuse attenuata, subdiaphana, fere lævi, cornea et cum incola fusco-atra; spira subconico-attenuata, ad summum perobtusa, fere subito tholiformi; apice minutissimo; anfractibus 5, quorum superiores 2 exigui, tertius relative major et ad lævam partem perventrosus, cæteri inflati, exacte cylindrici ac rapide descendentes et quasi subcontorti; ultimo mediocri, $1 / 3$ altitudinis leviter superante, inflato-rotundato, circa suturam perprofundam quasi subcanaliculato; apertura subverticali, ovata; peristomate continuo, recto, intus leviter incrassatulo; operculo late spirescente, rubro-sanguineo, in centro atro; alt. $31 / 2$, diam. $23 / 4$, alt. ap. $11 / 4$, lat. ap. 1 millim.

Oasis d'Oudref (Let.). Espèce rare.

\section{A. paradoxa Bourguignat, sp. nov.}

Testa vix rimata, oblongo-elongata, subdiaphana, non nitente, subtiliter striatula aut sublævi, smaragdino-cornea; spira producta, ad summum obtuse rotundata; apice minutissimo; anfractibus 5 irregulariter crescentibus quorum embryonales exigui, tertius amplus, ventrosus et cæteri magni, contorti, descendentes, pertumidi ac exacte cylindrici; sutura lineari usque ad quartum, dein profunda usque ad aperturam; ultimo relative mediocri, $1 / 3$ altitudinis leviter superante, rotundato, tumido; apertura subobliqua, subrotundata; peristomate continuo, recto, acuto, intus incrassatulo; operculo (ignoto); alt. 4, diam. 2 , alt. et lat. ap. æqualiter 1 1/2 millim.

Oasis d'Oudref (Let.). Espèce peu abondante.

\section{S 2.}

A. Latasteana Letourneux et Bourguignat, sp. nov.

Testa punctiformi-rimata, breviter conica, inferne ventrosa, diaphana vix nitidula, argute striatula, corneo-lacticina et sæpius limo viridescente inquinata; spira curta, conica, nihilominus ad summum obtusiuscula; anfractibus 5 convexis, regulariter usque ad ultimum, dein perve- 
lociter crescentibus, sutura parum impressa usque ad ultimum, deinde profunda, separatis; ultimo maximo, amplo, dimidiam altitudinis æquante, rotundato, superne circa suturam perprofundam planulato ac prope insertionem breviter descendente; apertura obliqua, ovata; peristomate continuo, recto, acuto, fragili; operculo (ignoto); alt. 4, diam. 3 , alt. ap. 2 , lat. ap. 1 1/2. millim.

Oasis d'Oudref (Let.). Rare.

\section{S 3.}

A. subsealaris Bourguignat, sp. nov. 188 2.

Testa rimata, ventrosa, elongato-acuminata, solida, opaca, parum nitida, subtiliter striatula, corneo-nigrescente; spira producta, acuminata, ad summum acuta; apice minutissimo; anfractibus 7 convexis, circa suturam profundam tumidulis quasi scalariformibus, regulariter crescentibus; ultimo majore, dimidiam altitudinis non æquante, convexo, superne prope suturam bene tumido; apertura subobliqua, ovata; peristomate continuo, obtuso, incrassato, ad basin leviter subpatulescente; operculo (ignoto); alt. 7 , diam. $31 / 2$, alt. ap. 3 , lat. ap. 2 millim.

Oued Gabès, à Gabès (Let.).

Le type a été découvert en Algérie, à Mokta-el-Oued, entre Djelfa et Boghar.

\section{A. Bythinopsis Bourguignat, sp. nov.}

Testa punctiformi-perforala, ventroso-oblonga, opacula, nitidula, sublevi, cornea; spira sat producta, regulariter acuminata; apice acuto, minutissimo; anfractibus 7 convexis, regulariter crescentibus ac sutura sat profunda separatis; ultimo dimidiam altitudinis non æquante, rentroso, ad aperturam non ampliore sed potius subcoarctato; apertura subobliqua, in directionem leviter obliquam a dextra ad sinistram ovata; peristomate continuo, recto, crasso; operculo atro, spirescente; alt. 5 1/2, diam. $31 / 2$, alt. ap. 2 1/2, lat. ap. 2 millim.

Cette espèce, qui ressemble beaucoup comme forme à la Bythinia Lusitanica de Castro, vit dans les eaux des environs de Gabès (Berthier).

\section{A. cyrniaca Mabille in Arch. malac. (fasc. iv, fév. 1869), p. 55.}

Cette Amnicole, découverte en Corse dans les environs de Bastia, a été recueillie par M. Doûmet-Adanson dans les cours d'eau du Bou-Hedma, entre Sfax et Gafsa.

A. waharica Bourguignat, sp. nov. 1879 et in Jus, Moll. viv. puils artés. de l'Oued Rir', p. 1 (sans descriplion), 1883.

Testa vix rimata, ventrosa, superne acuminata, subdiaphana, nitida, argute striatula, pallide corneo-lacticina; spira oblonga, sat producta, ad 
summum acuta; apice minutissimo; anfractibus 6 convexis, sat celeriter crescentibus, sutura impressa separatis; ultimo magno, dimidiam altitudinis æquante, rotundato, superne ad insertionem descendente; apertura sat obliqua, subrotundata; peristomate continuo, recto, intus incrassato ac leviter subpatulescente; operculo spirescente, aurantiaco, in centro atro; alt. 4 1/2-5, diam. 3 , alt. ap. $21 / 2$, lat. ap. 2 millim.

Cette espèce, constatée dans l'Oued Gabès, à Gabès (Let.), est très abondante en Algérie, notamment dans les eaux des puits artésiens de Sidi-Reched, de SidiSliman, de M'raier, etc., entre Biskra et Tugourt. Nous la connaissons également de la fontaine chaude de Biskra et de la source thermale du ruisseau des Zaatcha, dans le M'zab occidental.

L'A. Saharica existe aussi en Sicile, où elle a été trouvée dans l'Oreto, près de Palerme.

\section{$\$ 4$.}

A. Iuteola Bourguignat, Malac. Alg. II, p. 239, pl. XIV, f. 36-38, 1864 (Paludina luteola, Kuster, Palud. p. 44, pl. IX, f. 8-9, 1852).

Espèce abondante à Aïn Bou-Driès, à Gabès, à El-Kis, près Feriana, à Aïn esSnam (Let.), à Tozer (Doûm.-Ad.).

L'A. luteola est très répandue en Algérie, où elle a été constatée notamment aux environs d'Oran, à Aïn Sfa, au sud de Toukria, à Sebaïn-Aioun, dans le Sersou, à Djelfa, à Smendou, entre Philippeville et Constantine, ainsi que dans la fontaine de la plage de Bône.

\section{S5.}

A. Maceana Paladilhe, in Nouv. miscell. malac. (4* fase. 1869), p. 103, pl. V, f. $6-7$.

Le type de cette Amnicole existe, en Espagne, à Casa Autunez, près Barcelone; en Tunisie, cette espèce a été retrouvée aux environs de Gafsa (Doûm.-Ad.).

A. similis Bourguignat, Malac. Alg. II, p. 257 , pl. XIV, f. 28-30, 1864 (Cyclostoma simile, Draparnaud, Hist. Moll. Fr. p. 34, pl. I, f. 15, 1805 ; Paludina similis, Michaud, 1831; Bithinia similis, Dupuy, 1849).

Ruisseaux des jardins de Gafsa (Let. et Doûm.-Ad).

M. le professeur Issel (Ann. mus. civ. Genova, XV, p. 281, 1880) signale cetle espèce de Kebilli et de Mtuja [Metouïa] (Bell. 1875), ainsi que des alluvions de la Medjerda (Gestro, 1877).

M. le commandant Morlet, de son côlé, mentionne (Arch. miss. sc. $3^{\circ}$ série, VII, 1881, p. 395) une Amnicole, sous le nom de similis, des ruisseaux d'El-Hamma de Gabès, de Keriz, de l'Oued Akarit et de l'Oued Melah (André).

LiA. similis est une espèce fort répandue dans les contrées du sud de la France (le type se trouve aux environs de Cette), ainsi qu'en Espagne, aux Baléares et en Algérie. 
$\$ 6$.

A. pyenoeheilia Bourguignat, Malac. AIg. II, p. 241 , pl. XIV, f. 46-48, 1864.

El-Hamma des Beni-Zid, oasis de Gabès et d'Oudref, ainsi qu'à Mansoura, dans le Nefzaoua (Let.).

Une forme major à test un peu plus épais existe aux environs de Tozer (Let.).

L'A. pycnocheilia est une espèce algérienne répandue çà et là dans les eaux du sud des provinees d'Alger et de Constantine(1).

A. Pomariensis Letoumeur, Excurs, malac. Kabylie, in Ann. Malac. I, p. $\mathbf{3}_{17}, 1870$.

Le type de cette Amnicole se trouve aux cascades du Sefsef, près de Tlemcen, en Algérie.

Celte espèce est excessivement abondante dans l'Oued Gabès et dans l'Aïn Zerig, près de Gabès (Let. et Doûm.-Ad.), ainsi qu'aux alentours de Ketenna (Let.).

\section{s 7.}

1. Dupotetiana Bourguignat, Malac. Alg. II, p. 244 , pl. XIV, f. 31-35, 1864 (Paludina Dupotetiana, Forbes, Moll. Alg. in Ann. nat. hist. or Mag. zool. p. 234 , pl. XII, f. 3,1838$)$.

Amnicole commune dans la vase des ruisselets de la source d'Aïn Temoguet, près de Djado, dans l'Ouadi Arhlân (Duveyrier). Ruisseaux du Bou-Hedma, entre Sfax et Gafsa (Doûm.-Ad.), de l'Aïn Bou-Driès et de l'Aïn du marabout de Sidi Abd-er-Ralıman el-Garsi, ainsi que ceux de l' lïn Medeker dans l'Enfida (Let.).

Celte espèce a été encore constatée par M. le professeur Issel dans les alluvions de la Medjerda (Gestro, 1877) et par M. le commandant Morlet, dans les eaux d'El-Hamma de Gabès, de l'Ain Oudref, de l'Oued Melah et de la sonreo thermale de Keriz (André).

L'A. Dupotetiana est une coquille très répandue en Algérie. Elle existe également en Sicile, à Madonie, d'où nous l'avons reçue sous le nom d'A. sicula (Adami).

S 8.

A. sterea Bourguiguat, sp. nov. 1868.

Tesla oblique rimata, ventroso-tumida, globulosa, sat crassula, opaca, striatula aut levigata, pallide corneo-viridescente aut potius plerumque limo atro inquinata; spira brevi, tumido-rotundata, globulo parvulo simili ac inter penultimum et ultimum, propler ultimi ad aperturan coarctationem, quasi leviter strangulata; apice obtuso; anfractibus $4-5$ convexis, rapide crescentibus, sutura lineari usque ad penultimi dimidiam, dein profunda separatis; penultimo amplo, tumido-perventroso; ultimo me-

(1) Cette espèce a encore été constatée à Vendrell, près de Barcelone, en Catalogne. 
diocri, dimidian altitudinis æquante, rotundato, ad aperturam non ampliore sed potius quasi leviter coarctato; apertura verticali, fere sphæricȧ; peristomate continuo, reclo, intus incrassatulo; operculo spirescente, atro; alt. 3 , diam. 2 , alt. ap. 1 1/2, lat. ap. 1 i/4 millim.

Celte espèce, découverte en $186_{7}$ à Aïn Sfa, source entre Toukria et SebaïnAioun, dans le Sersou, a été retrouvée en Tunisie, dans les eaux de Limaguès; dans le Nefzaoua (Let.).

\section{A. globalina Letourneux et Bourguiguat, sp. nov.}

Testa-vix rimata, globosa, subdiapliana, nitida, subtiliter striatula, corneo-rubella; spira curta, obtusa; anfractibus 4 convexis, celeriter crescentibus, sutura impressa, inter penultimum et ultimum profunda, separatis; ultimo majore, dimidiam altitudinis leviter superante, rotundato; apertura oblicqua, subrolundato-ovata; peristomate continuo, recto, acuto; operculo spirescente, atro; alt. 3 , diam. 2 , alt. ap. $13 / 4$, lat. ap. 1 1/4 millim.

Tala, El-Kis, près de Feriana, ainsi que dans le bassin du temple du Djebel Zaghouan (Let.).

\section{A. Doumeti Letournenx et Bourguighat, sp. nov.}

Testa obtecte rimata, curta, globoso-perventrosa quasi sphærica, diaphana, nitidissima, argutissime striatula, corneo-rubella; spira brevissima, rotundato-perobtusa; anfractibus 4 convexis, rapide crescentibus, sutura profunda, quasi canaliculata, separatis; ultimo maximo, fere totam altitudinem æquante, rotundato; apertura subobliqua, valde aperta, ovata; peristomate continuo, recto, acuto; margine columellari robusto, super rimam reflexo; operculo (ignoto); alt. 2 1/2, diam. $21 / 4$, alt, ap. 2 , lat. ap. $13 / 4$ millim.

Cours d'eau, près de Tala (Let.).

A. pyenolena Bourguignat, Malac. Alg. II, p. 242 , pl. XIV, f. 42-45, 1864 .

Cette espèce algérienne a été conslatée à Nefta, à la source thermale de Keriz (André), aux environs de Kérouan, dans le bassin du temple du Djebel Zaghouan, enfin dans les ruisseaux, près de Ketenna et de Limaguès (Let.). .

A. Letourncuxiana Bourguignat, Malac. Alg. II, p. 248 , pl. XIV, f. 58-60, 1864.

Cette Amnicole a été signalée par M. le professeur Issel (Ann. mus. civ. Genova, $\mathrm{XV}, \mathbf{1} 881$, p. 281), dans les cours d'eau de Kebilli (Bell. 1875 ).

\section{A. Barrattei Letourneux et Bourguignat, sp. nov.}

Testa minuta, vix rimata, ovata, crassula, opaca, non nitente, lævi, semper limo viridulo induta; spira brevi, obtuse attenuata; anfractibus 
4 convexiusculis, rapide crescentibus, sutura lineari usque ad ultimum, dein profunda, separatis; ultimo majore, dimidiam altitudinis leviter superanie, convexo, superne prope insertionem soluto et leviter descendente; apertura subobliqua, ovata; peristomate continuo, recto, acuto, incrassatulo ac ad basin subpatulo; operculo atro; alt. 2 1/2, diam. 2 , alt. ap. 1 1/2, lat. ap. 1 millin.

Espèce très abondante dans les ruisseaux du Nefzaona et notamment aux environs de Nefta (Let.).

A. perforata Bourguignat, Malac. Alg. II, p. $\mathbf{4} 46$, pl. XIV, f. $49-57,1864$.

Cette Amnicole d'Algérie a été retrouvée au Djebel Zaghouan, dans le bassin du temple (Let.).

\section{A. ragia Letourneux et Bourguignat, sp. nov.}

Testa minutissima, non rimata, ovato-globosa, crassula, opacula, parum nitida, lævi, cornea; spira brevi, globosa, nihilominus ad summum sat acutiuscula; anfractibus 4 convexis, rapide crescentibus, sutura impressula separatis; ultimo magno, dimidiam altitudinis æquante, rotundato, ad aperturam soluto et ad insertionem abrupte deflexo; apertura obliqua, sphærica; peristomate continuo, crasso, obtuso ac leviter patulo; operculo fusco; alt. 2 , diam. 1 1/2, alt. et lal. ap. æqualiter 1 millim.

Environs de Keriz, dans le Djérid (Let.).

\section{BYTHINELLA ${ }^{(1)}$.}

Les Bythinelles de l'Algérie, en y comprenant celles de la Tunisie, sont au nombre de quatorze.

Ces espèces appartiennent à quatre groupes distincts :

$1^{\circ}$ A celui de la B. viridis,

Bythinella desertorum Bourg. 1862, Algérie;

$2^{\circ}$ A celui de la B. Lisropsis,

B. limnopsis Let. et Bourg., sp. nov. Tunisie;

$3^{\circ}$ A celui de la B. sruroricana,

B. mauritanica Bourg. $187^{2}$, sp. nov., Algérie et Tunisie;

B. microcochlia Let. et Bourg. sp. nov. Tunisie;

$4^{\circ}$ A celui des Fradenfeldia,

B. sordida Bourg. 1862 (Paludina sordida Küster, 1852), Algérie;

B. elachista Bourg. 1862, Algérie;

(1) Moquin:Tandon, 1851 . 
B. Challameliana Bourg. 186h, Algérie;

B. Tlemcenensis Bourg. 1877, sp. nov. Algérie;

B. punica Let. et Bourg. sp. nov. Tunisie;

B. Berthieri Bourg. 1877 , sp. nov. Algérie;

B. Reboudi Bourg. 1866, Algérie;

B. nana Issel, 1880 (Paludina nana Terver, 1839), Algérie et Tunisie;

B. seminium Bourg. 1862 (Palıdina seminium Morelet 1851), Algérie,

Et B. microsoma Bourg. 1877 , sp. nov. Algérie.

Jusqu'à ce jour, on n'a pu découvrir des Maresia dans la Régence.

S 1.

B. limnopsis Letourneux et Bourguignat, sp. nov.

Testa rimata, oblonga, limnæiformi ac Limncece truncatule simili, opacula, nitida, lævi, castanea; spira sat producta, subacuminata; anfractibus 5 convexis, rapide crescentibus, sutura sat profunda separatis; ultimo majore, dimidiam altitudinis non æquante, convexo; apertura subobliqua, oblonga; peristomate continuo, recto, incrassatulo ac patulo præsertim inferne; margine columellari validiore, recto ac reflexo; operculo atro-castaneo; alt. $21 / 2$, diam. 1 1/2, alt. ap. $11 / 4$, lat. ap. $3 / 4$ millim.

Cette belle espèce, qui ressemble tout à fait en petit à la Limncea truncatula, vit dans le bassin du temple du Djebel Zaghouan (Let.), ainsi que dans quelques sources aux environs de Kérouan (Doûm.-Ad.).

\section{s 2.}

B. mauritanica Bourguignat, sp. nov.

Testa leviter rimata, producto-oblonga, ad penultimum sat ventrosa, opacula, non nitente, lævi, cornea, sæpe limo inquinata ; spira subelongata, regulariter attenuata, ad summum obtusiuscula; anfractibus 5 rotundatis, sat regulariter crescentibus, sutura profunda separatis; ultimo rotundato, $2 / 5$ altitudinis æquante; apertura subobliqua, ovato-rotundata; peristomale continuo, recto, acuto; operculo atro; alt. 5 , diam. $21 / 4$, alt. ap. 2 , lat. ap. 1 1/2 millim.

Cette Bythinelle, de la source du Hamma, près Alger, et des ruisseaux des jardins de Mostaghanem, a été retrouvée bien typique, dans le bassin du temple du Djebel Zaghouan (Let.).

B. mierocochlia Letourneux et Bourguignat, sp. nov.

Testa minima, rimata, subconica, inferne ventrosa, subdiaphana, non nitente, lævi, cornea ac sæpius limo atro induta; spira breviter attenuata; anfractibus 4 rotundatis, sat celeriter crescentibus, sutura in. 
pressa separatis; ultimo majore, dimidiam altitudinis non aquante, rotundato; apertura obliqua, ovata; peristomate non continuo, recto, acuto, fragili; margine columellari superne dilatato ac super rimam reflexo; operculo atro; alt. $13 / 4$, diam. $3 / 4$, alt. ap. $3 / 4$, lat: ap. $1 / 2$ millint.

Cette microscopique espèce est excessivement abondante dans l'Oued Melalı, près Oudref (Let.); on la rencontre encore, mais plus rarement, sur les herbes des eaux saumâtres, près de la Goulette (Pechaud).

\section{$\$ 3$.}

B. punica Letourneux et Bourgruignal, sp. nov.

Testa rimata, oblonga, subdiaphana, nitida, lævi, castanea; spira oroidea; anfraclibus 4 convexis, irregulariter crescentibus (embryonales 2 exigui, cateri relative ampli et convexiores), sutura inter supremos lincari, inter ultimos impressa, separalis; ultimo majore, dimidiam altitudinis aquante, convexo; apertma subobliqua, ovata; peristomate continuo, recto, acuto, ad marrinem columellarem late reflexo; operculo (ignoto); alt. 2 , diam. 1 1/2, alt. ap. 1, lat. ap. $1 / 2$ millim.

Sources, dans le domaine de l'Enfirla (Let.).

E. nana Issel, Moll. croc. Violante in Ann. mus. civ. Genova, XV, 1880, p. 280 (Paludina nana, Terver, Moll. nord de l'd frique, p. 37, pl. IV, f. 20-21, 1839).

Cette Bytlinelle minuscule, si répaudue en Algórie, a été constatée en Tunisie dans les alluvions de la Mledjerda (Gestro, 1877), dans le bassin du temple du Djebel Zaghouan, ainsi que dans le domaine de l'Enfida, à Ain Medeker et dans la gorge de Zaktoun à la source de l'Oued (Let.).

\section{PALUDESTRINA ${ }^{(1)}$.}

Sous ce nom générique, nous comprenons toutes les petites Paludinidées de forme allongée à spire plus ou moins acumince, qui ont été recueillies dans les eaux salées, douces ou saumâtres, parce qu'il nous a été impossible d'établir une ligne de démarcntion entre les espèces d'eau douce (Littoridina, olim Hydrobia) et celles des eaux salées on muriatisées (Paludestrina). Nous les avons donc toutes placées sous l'appellation de Paludestrina, qui date de $183_{9}$, puisque celle d'Hydrobia, bien quantérieure $(1821)$, est inadmissible pour cause de double emploi.

P. Peraudieri Bourguignat (Hydrobia Peraudieri, Bourg. in Spicil. Malac. p. 108 , 1862 , et Paléont. Alg. p. 94, pl. V, f. 12-15, 1862, et Malac. Alg. II, p. 227 , pl. XIV, f. 5-8, 1864).

Lac Kelbya, environs de Tozer, ruisseaux près Debabcha, dans le Nefzaoua (Let.).

(1) Ale. d'Orbigny, 1839 . 
On rencontre dans les ruisselets de l'oasis d'Oudref de magnifiques échantillons.

Le type de la P. Peraudieri vit dans le gouffe de la fontaine chaude de Biskra.

P. arenaria Bourguignat (Hydrobia arenaria Bourg. in Spicil. malac. p. 111, 1862, et Malac. Alg. II, p. 231 , pl. XIV, f. 14-16, 1864).

Nous ne signalons cette espèce que sur l'autorité de M. Je commandant Morlet, qui l'indique (in Arch. miss. sc. $3^{\mathrm{e}}$ série, VIII, 1881, p. $39_{9} 5$ ) d'El-Hamna de Gabès, de Keriz, de Debabcha et de l'Oued Melah (André). Quant à nous, nous ne connaissons pas cette Paludestrine de la Tunisie, dont le type, comme on le sait, se trouve dans les fossés de Mégarin, au nord de Tuggurt.

P. Chospitali Mabille, Cat. Paludestrines de France, p. 2, 1877 .

Cette espèce, que l'on rencontre çà et là sur les côtes océaniennes de France, d'Espagne et de Portugal, vit également dans le lac Bahirah, près de Tunis (Pechaud).

\section{P. eucyphogyra Bourguignat, Spec. noviss. $\mathrm{n}^{\circ} 86,1876$.}

Cette belle Paludestrine, qui n'était connue que des côtes de France, a été retrouvée sur les bor'ds du lac Kelbya (Let.).

P. acerosa Bourguignat (Hydrobia acerosa Bourg. in Spicil. malac. p. 109, 1862, et Malac. Alg. Il, p. 228, pl. XIV, f. 9-10, 1864).

Ruisseau d'El-Hamma de Gabès (André), rives du lac Bahirah, près de Tunis, environs de Monastir, cours d'eau du Nefzaoua, non loin de Kebilli (Let.).

\section{P. meca Letourneux et Bourguignat, sp. nov.}

Testa vix rimata, elongalo-turriculata, subdiaphana, nilida, lævi aut argulissime striatula, vitracea ad aperturam infumata; spira producta, regulariter attenuata, ad summum obtusiuscula; anfractibus 7 convexis (quorum supremi 3 exigui arctique, cæeteri convexiores ac rapidius crescentes), sutura impressa separatis; ultimo mediocri, $1 / 3$ altitudinis æquante, rotundato; apertura verticali, subovato-rotundata; peristomate non continuo, recto, acuto, nigrescente; margine columellari super rimulam reflexo; operculo atro; alt. 6 , diam. 2, alt. ap. 2, lat. ap. 1 1/4 millim.

Oued Gabès, à Gabès et ruisseaux de l'oasis d'Oudref (Let.).

On rencontre, dans le Nefzaoua, à Limaguès et à El-Goléa (Let.) une variété d'une taille un peu moins allongée.

P. Cossoni Letourieux et Bourguignat, sp. nov.

Testa non rimata, elongata, cylindrica usque ad ultimum et dein ventroso-inflata, subopacula, parum nitente, subtiliter striatula, unifor- 
miter cornea; spira producta, ad summum altenuala, in medio cylindrica; anfractibus 8 convexis (quorum supremi 3 exigui, mediani cylindrici et ultimus fere subito relative amplissimus ac tumidissimus), sutura ad summum impressula, dein magis magisque usque ad aperturam impressa, separatis; ultimo relative magno prasertim in amplitudine, $1 / 3$ altitudinis æequante, rolundato; apertura verticali, ovata; peristomate recto, acuto, non continuo (margines callo juncti); margine externo antrorsum leviter arcuato; operculo corneo; alt. 5, diam. 2, alt. ap. 1 1/2, lat. ap. 1 millim.

Cette singutière Paludestrine vit autour de Keriz (Let.).

\section{P. mecyna Letournethx et Bourguignat, sp. nov.}

Testa non rimata, elongata, subopacula, sat translucida, nitida, subtiliter striatnla, obscure cornea aut corneo-subviridula; spira producta, regulariter attenuata, plerumque truncata aut decorticata; anfractibus 8 conrexiusculis, lente crescentibus, sutura lineari, nihilominus impressula separatis; ultimo mediocri, $1 / 2$ altitudinis non æquante, convexo; apertura verticali, ovata; peristomate recto, acuto, fere continuo; margine externo antrorsum fere recto; operculo luteo; alt. 5 1/2, diam. 2 , alt. ap. 2 , lat. ap. 1 1/4 millim.

Dans le Nefzaoua, aux environs de Kebilli (Let).

\section{P. oxitata Letourneux et Bourguignal, sp. nov.}

Testa non rimata, conico-elongata, inferne inflata, translucida, nitida, subtiliter striatula, opalina; spira prodıcta, regulariter acuminata, ad sunnmun acutata; anfraclibus 8 convexiusculis, lente usque ad ultimum crescentibus, sutura sublineari separatis; ultimo ad initium amplo, inflato, ad aperturam leviter diminuto, convexo; apertura verticali, oblonga, superne sat angulata; peristomate non continuo, recto, fragili, ad marginem columellarem late reflexo; operculo (ignoto); alt. 5, diam. 2 1/4, ailt. ap. 2 , lal. ap. 1 millim.

Cette espèce algérienne, connue de la fontaine thermale du ruisseau des Zaatcha, dans le M'zab occidental, vit, en Tunisie, dans les ruisseaux d'arrosement des environs de Kebilli (Let.).

P. procerula Paladilhe, in Nouv. miscell. malac. p. 131 , pl. V, f. $24-25$ ( $4^{\circ}$ fasc. 1869), et in Rev. et Mag. zool. p. 322, 1869 , et in Ann. Malac. I, 1870, p. 239 , et Mabille, Cat. Paludestrines de France, p. 7, 1877 .

Cetle espèce, des côles de la France, de l'Espagne et de l'Algérie, a été recueillie en Tunisie dans les Jagunes de l'Oued Chebia et de Monaslir (Let.).

P. acuta Paladilhe, in Ann. Malac. J, 1870, p. 238 , et Mabille, Cat. Daludest. Fr. 
p. 8, 1877 (Cyclostoma acutum, Drap. Moll. Fr. p. 40, pl. I, f. 23, 1805 ; Paludina acuta, Michaud, complém. p. 100, 1831).

Lagune au sud de Monastir (Let.); lac Bahirah, près de Tunis, où elle est abondante (Pechaud).

Cette Paludestrine est fort répandue sur les côtes méditerranéennes de France et d'Espagne.

P. Duveyxieri Bourguignat, 1877 (Hydrobia Duveyrieri, Bourg. Moll. Sahara in 'Touareg du nord, p. 17, pl. XXVII, f. 18-20, 1864, et Malac. Tunis, p. 33, f. $38-40,1868$ ).

Environs de Keriz, où se trouve fe type (Duveyrier), Kebilli et Mtuja [Metoüa] (Bell. 1875 ); Oued Akarit (André), ruisseaux de l'oasis de Gabès, notamment à Ras El-Oued, enfin dans le Nefzaoua, à Aïn Tanorga, Mansoura et Debabcha (Let.).

P. Coutagnei Bourguignat, sp. nov. 1881 , in Coutagne, Ét. Faune malac. bassin du Rhône, p. 26, 1881 .

Cette espèce, de l'étang de Berre, près de Marseille, a été retrouvée à l'ìle de Tabarka et dans l'Oued Serrag (Let.).

P. gracillima Bourguignat, Spec. noviss. $\mathrm{n}^{0} 9^{3}, 1876$, et Mabille, Cat. Paludest. Fr. p. 8,1877 .

Marais entre Utique et Porto-Farina (Let. et Pechaud).

Le type de cette espèce se rencontre dans les lagunes des côtes du Roussillon.

P. vitracea Letourneux et Bourguignat, sp. nov.

Testa vix rimata, breviter conico-pyramidali, inferne tumido-ventrosa, diaphana, fragili, nitida, lævi et vitraceo-albescente; spira rapide acuminata; apice minimo; anfractibus 7 rotundatis, lente crescentibus, sutura profunda separatis; ultimo magno dimidiam altitudinis fere æequante, inflato ac bene rotundato; apertura subobliqua, ovata; peristomate non continuo, recto, acuto; margine externo recte leviter retrocedente ; marginibus valde approximatis; operculo diaphano; alt. 4, diam. 2, alt. ap. $13 / 4$, lat. ap. 1 millim.

Oued Serrag (Let.).

P. pachygaster Paladilhe, Nouv. miscell. malac. p. 132 , pl. V, f. $26-27$ ( $4^{\circ}$ fasc.), 1869 .

Cette espèce sicilienne a été constatée dans une lagune, au sud de Monastir (Let.).

P. viridescens Issel, Moll. croc. violante in Ann. mus. civ, Genova, XV, p. 280,1880

(Paludina viridescens, Calcara, Moll. Siciliæ, p. 27, pl. IlI, f. 17, 1845 ; Leachia viridescens, Monterosato, nomencl. gen. e spec. conch. medit. p. 70, 1884).

Bords du lac Bahirah (Gestro et Pechaud). 
P. arenarum Bourguignat, Spec. noviss. $n^{\bullet} 97,1876$, et Mabille, Cat. Paludest. Fl. p. 9,1877 .

Celte espèce française vil en Tunisie, entre Klibia el Zougag (Let.).

P. Minoriciensis Bourguignat (Peringia Minoriciensis, Paladilhe, Desc. nouv. Esp. Moll. p. 14, f. 27-39, 1875 ).

Canal salé près Menzel-Temin; lagune près l'Oued Lebna, entre Nebeul el Klibia (Let.).

Le type vit aux Baléares.

P. leneumiera Bourguignat, Spec. noviss. $\mathrm{n}^{\circ} 99,1876$, et Mabille, Cal. Paludest. Fr. p. 9,1877 .

Celle espèce française, des côtes du Roussillon, se reucontre assez fréquemment dans l'Oued Serrag et dans l'Oued Melah, près Oudref (L̨el.).

\section{PERINGIA (1).}

Les espèces de ce grenre sont des Mollusques qui vivent indifféremment, ainsi 'fue les Paludestrines, dans les eaux marines, snumâtres ou même faiblement nurialisées. C'est nolamment sur le bord des còles, à l'embouchure des fleuves ou dans les marais salants, qu'on les rencontre presque toujours. En Tunisie, fail digne de remarque, les Péringies n’ont encore été constatées que dans des cours d'ean ou des ruisseaux d'arrosement de l'intérieur des terres, dans le Nefzaoua, au sud du grand Choll salé El-Fedjedj.

La présence de Péringies dans lintérieur des terres a déjà élé qualre fois signalée :

1. I Saint-Amour, dans le Jura (Per. Gallica, Paladille, in Ann. sc. nat. 1874);

$2^{\circ}$ A Bex, dans le Valais (Per. Helvetica, Bourg. spec. 11oviss., p. 58, 1876);

$3^{\circ}$ Dans une mare, à a kilomètres de Remnes (Per. Lelourneuxi, Bourg. spec. noviss. p. 59,1876 );

$4^{\circ}$ Dans les alluvions de l'Ëbre, près Saragosse (Per. Hispanica, Servain, Moll. Lspagne, p. 152,1880 ).

II. Jules Mabille, en 1877 , a donné, dans la Revue el Magasin de zoologie, une Étude sur les Péringies de nos possessions du nord de l'A frique. Nous renvoyons à cet excellent travail pour la connaissance de ces espèces.

P. puniea Letourneux el Bourguignat, sp. nov.

Testa vix rimata, oblongo-elongata aut elongato-subconoidea, opaca aut opacula rel aliquando subdiaphana, non nitente, argule striatula, raro subvitraceo-albescente, sed sæpius compacto-cretacea el sæpissime limo viridulo induta; spira producta, regulariter acuminata; anfractibus

(1) Paladilhe, 1874 . 
7 convexiusculis, lente crescentibus, sutura sublineari, inter penultimum et ultimum impressa, separatis; ultimo $2 / 5$ altitudinis æquante, convexo ; apertura ad basin antrorsum provecta, exacte ovata; peristomate continuo, recto, obtusiusculo, intus crassiusculo et subpatulescente; margine externo antrorsum regulariter arcuato; operculo (ignoto); alt. 5, diam. $21 / 2$, alt. ap. 2 , lat. ap. 1 1/2 millim.

Cours d'eau aux alentours de Kebilli, dans le Nefzaoua (Let.). Espèce très abondante.

P. paradoxa Letourneux et Bourguignat, sp. nov.

Testa aperte rinata, irregulariter oblonga, leviter streptaxiformi, sinistrorsum quam ad dextram convexiore, opaca, non nitente, semper limo crelaceo-riridulo induta; spira producta, acuminata, nihilominus ad summum obtusiuscula; anfractibus $7-8$ ( superiores convexiusculi; ultimi convexiores et ad sinistram valde ventrosi), irregulariter (supremi arcti; penultimus et ultimus velociter augentes) crescentibus, sutura subimpressa usque ad aperturam separatis; ultimo relative mediocri, $2 / 5$ altitudinis non æquante, rotundato et superne prope insertionem descendente; apertura verticali, ovata ac ad dextram valde versa; peristomate continuo, recto, intus albo-incrassato ac patulo; margoine externo antrorsum vix arcuatulo; operculo nigrescente; alt. 5 1/4, diam. 3 , alt. ap. 2 , lat. ap. 1 1/2 millim.

Cette Péringie, remarquable par son ouverture très portée du côté droit et par son test streptaxiforme plus bombé du côté gauche que du côté droit, vit dans quelques ruisselets près Kebilli, où elle semble peu commune (Let.).

P. tumida Bourguignat, Spec. noviss. p. 66, 1876 .

Cette espèce, qui n'était connue que de l'embouchure de la Macta, près d'Oran, a été retrouvée parfaitement typique dans le Nefzaoua, aux environs de Kebilli (Let.).

\section{P. solitaria Letourneux et Bourguignat, sp. nov.}

Testa rimata, relative curta, ventroso-conica, opacula, non nitente, argutissime striatula, semper limo viridulo-cretaceo induta; spira conica, mediocriter producta, ad summum acula; anfractibus 6 convexiusculis, regulariter usque ad penultimum, dein velociter crescentibus, sutura impressa separatis; ultimo sat majore, dimidiam altitudinis fere æquante, convexo-rotundato; apertura verticali aut ad basin antrorsum leviter provecta, ovata; peristomate continuo, recto, incrassatulo ac subpatulescente; margine externo antrorsum subarcuato; operculo (ignoto); alt. 4 1/4, diam. 2 1/2, alt. ap. 2 , lat. ap. 1 1/2 millim.

Ivec la précédente, aux environs de Kebilli (Let.). 
P. ndmirabilis Lelourneux et Bourguignat, sp. nov.

Testa subrimata, sat brevi, ventroso-ovata, opaca, non nitente, limo viridi semper induta; spira breviter attenuata ac ad summum peracute prolongata; apice minutissimo, cylindrico, acutato ac quasi super spiram superposito; anfractibus 7 (superiores exigui, arcti; ultimi tumidi, rapide crescentes), sutura impressula separatis; ultimo majore, convexo, dimidiam altitudinis fere æquante, superne prope insertionem leviter descendente; apertura verticali, ovata; peristomate continuo, recto, alboincrassato; margine externo superne sinuoso, dein in medio antrorsum leviter arcuato; operculo atro; alt. 4 1/2, diam. 2 1/2, alt. ap. 2 , lat. ap. 1 1/2 millim.

Cette Péringie, si reconnaissable à sa forme écourlée, ventrue, atténuée en une pointe acérée qui a l'air d'être juxtaposée sur la spire, a été rencontrée également dans les cours d'eau du Nefzaoua, autour de Kebilli (Let.).

\section{IMELA N I AE.}

\section{MELANIA (').}

M. Iuberculata Bourguignal, Cat. rais. Moll. orient. p. 65,1853 et Malac. Alg. Il, p. 251, pl. XV, f. 1-11, 1864 (Nerita tuberculata, Müller, Verm. hist. II, p. 191, 177 . Melania fasciolata des auteurs).

Cette espèce cosmopolite est des plus abondantes dans toutes les eaux des régions basses de la Tunisie, notamment dans celles des oasis; aussi jugeons-nous superflu d'indiquer les nombrenses localités où elle a été recueillie.

\section{MELANOPSIS ${ }^{(2)}$.}

\section{S 1.}

M. olivula Lelourneux et Bourguignal, sp. nov.

Testa oliveformi, superne obtuso-attenuata, crassa, castanea, vel sordide limo inquinata aut plerumque erosa, sublævigata, ad aperturam striatula; spira curta, obtusa; anfractibus 4 arcte spiratis, sutura impressa separatis; ultimo inaximo, fere totam altitudinein efformante, convexo, nililominus ad tertiam partem superam leviter subconcaviusculo, ad basin prope columellam cristato (crista prominens); apertura verticali, pyriformi, superne in rimulam longe angulato-prolongata, ad partem inferam in directionem subdescendentem externe leviter expansa, intus castaneo-micante; columella brevi, arcuata, inferne abrupte truncata (sinus truncaturæ profundus); peristomate acuto, simplici; margine ex-

(1) Lamarck, 1799 .

(1) Ferussac, 1807 . 
terno antrorsum vix arcuatulo et leviter sinuoso; marginibus callo ad insertionem crasso ac tuberculoso junctis; alt. 12, diam. 5, alt. ap. 9 millim.

Cette espèce, remarquable par la brièveté de sa spire, par l'énorme développement de son dernier tour, non moins que par sa forme olivoïde, a été découverte à Nefta, dans les canaux d'arrosement (Let.) et à El-Hammam, près Tozer (Doûm.-Ad.).

M. Doumeti Letourneux et Bourguignat, sp. nov.

Testa curta, oblongo-ovoidea, sat ventrosa, opaca, crassula, atra, subtiliter striatula aut in ultimo passim obsolete subcostulata; spira brevi, obtusa (apex semper erosus); anfractibus 4 convexiusculis, sutura lineari separatis; ultimo relative maximo, fere totam altitudinem efformante, convexo, ad basin leviter cristato; apertura subverticali, oblongo-pyriformi, superne longe angulato-prolongata, ad partem inferiorem leviter dilatata aut aliquando subattenuata, intus nitidissima ac atro-violacea; columella brevi, recta aut subarcuata, ad basin abrupte truncata (sinus truncaturæ profundus); peristomate cultrato; margine externo antrorsum regulariter arcuatulo; marginibus callo, ad insertionem pervalido ac tuberculum simulante, junctis; alt. 11, diam. 6, alt. ap. 9 millim.

Cette Mélanopside, dédiée à M. Doûmet-Adanson, est abondante dans les eaux de Gafsa; on la rencontre encore, mais plus rarement, dans celles de Tozer et de Nefta (Let.).

M. sahariea Bourguignat, 1882 et in Locard, Malac. lac Tibériade, p. 72, 1883 et Bourg. Hist. Mélan. syst. europ. p. 77, 1884 (Melanopsis maroccana, var. saharica, Bourg. Malac. Alg. II, p. 260 , pl. XII, f. 9, 10, 13, 13 et 14 [excl. fig. 11], 1864).

Cette petite espèce, répandue dans les eaux des oasis, au sud de Biskra, a été rencontrée en Tunisie, dans les canaux d'arrosement, aux environs de Mansoura, dans le Nefzaoua (Let.). Les échantillons de cette localité sont d'une taille un peu supérieure à celle des échantillons des eaux algériennes.

M. praemorsa (1) Bourguignat, Hist. Mélan. syst. europ. p. 78,1884 (Buccinum præmorsum, Linnceus, Syst. nat. [x ed. 1758 ], p. 740 , et Buccinum prærosum, Linnoeus, Syst. nat. [xil ed. 1766 ] $\mathrm{n}^{\circ} 1203$. Melanopsis prærosa, Hanley, Ipsa Linn. conch. p. 255 , pl. II, f. 5, 1855).

Le type de cette espèce, qui ne nous était connue que de trois localités: Séville, Lorca (Espagne) et Saïda (Algérie), a été retrouvé, en Tunisie, à Nefta (Let.), où il est fort rare.

Non Melanopsis præmorsa ou prærosa de tous les auteurs. 
\$2.

M. laevigat:a Lamarck, Anim. s. vert. VI ( $\left.2^{\circ} \mathrm{p}^{\text {tie }}\right)$, p. 168 (excl. syn.) 1829 , et Bourg. Hist. Mélan. syst. europ. p. 83, 1884.

Cette Mélanopside, répandue dans un grand nombre de cours d'eau du bassin méditerranéen, notanıment dans les eaux des régions orientales, vit à Mansoura dans le Nefzaoua, ainsi que dans l'Oued Gabès (Let.). Les échantillons de cet Oued appartiennent à la variété minor, signalée (p. 85) dans l'Histoire des Mélaniens du système européen.

M. episema Bourguignat, Hist. Mélan. syst. europ. p. 88, 1884.

Canaux d'arrosement de Mansoura (Let.), de Gabès et de Tozer (Doûm.-Ad.). Variété minor aux alentours de Gafsa (Let.).

M. Latastei Letommeur et Bourguignat, sp. nov.

Testa obtuse oblonga, sat curta, opaca, crassula, striatula, atra aul plerumque erosa; spira breviter atlenuata (apex sat acutiusculus); anfractibus \& convexiusculis, regulariter crescentibus, sutura fere lineari separatis; ultimo maximo, $2 / 3$ altitudinis aquante, leviter convexiusculo aut potius planulato, inferne convexiore ac relative turgidulo, superne ad insertionem descendente; apertura verticali, ovata, superne in rimulan longe angulato-prolongata, basi ad parten externo-inferiorem dilatata ac columellam relative valde transeunte, intus atro-micante; columella brevi, arcuatula, ad basin abrupte truncata (sinus truncaturx profundus); peristomate recto, acuto; margiuc externo antrorsum parun arcuatulo; marginibus callo, ad insertionem tuberculum validum forınante, junctis; alt. 12 , diam. 6 , alt. ap. 8 millim.

Abondante dans les caux des environs de Nefta (Let.). Moins fréquente dans l'Oued Gabès (Let.) et aux alentours de Tozer (Doun.-Ad.).

I. maahiea Bourguignat, Hist. Mélan. syst. europ. p. 89, 1884 .

Cette espèce, commune dans les oasis du sud de la province de Constantine, a été retrouvée typique aux environs de Tozer (Let.).

A Keriz (Doûm.-Ad.) et à El-Goléa, dans le Nefzaoua (Let.), on rencontre assez abondamment une forme minor.

M. minutula Bourguignat, Hist. Mélan. syst. europ. p. 92, 1884 .

Cette petite Mélanopside asiatique, qui, dı reste, avait été déjà constatée au puits artésien de Tamerna-Kedima, dans le Ziban (Algérie), vit en Tunisie dans les ruisseaux du Djebel Bou-Herlma (Doûm.-Ad.).

\section{S 3 .}

M. coupha Bourguignat, Hist. Mélan. syst. europ. p. 94, 1884.

Eaux chandes, au nord du Chott El-Djerid (Dnveyrier). 
$\$ 4$.

M. maroceana Bourguignat, Hist. Mélan. syst. europ. p. 99, 1884 (Buccina maroccana, Chemnitz, Conch. cab. XI, p. 285 [pars] et pl. CCX, f. 2078 et 2079 seulement, 1795 ; Melanopsis maroccana [pars], Bourg. Malar. Alg. II, p. 267 , pl. XV, f. 12,13 et 14 seulement, 1864).

Cette espèce, si répandue en Algérie, vit en Tunisie, aux environs de Gabès, et dans les eaux du Djebel Bou-Hedma (Doûm.-Ad.), oì l'on rencontre également une variété minor.

\section{\$5.}

M. Seignettei Bourguignat, Hist. Mélan. syst. europ. p. 103, 1884 (mel. Maresi [non Bourg.], Kabelt, Iconogr. f. 1884, 1880).

Environs de Tozer (Doûm.-Ad.), où l'on trouve également nne très pelite variété dont les échantillons n'ont pas plus de 10 millimètres de haut sur $41 / 2$ de. diamètre.

$\$ 6$.

M. belonidaea Bourguignat, Hist. Mélan. syst. enrop.p. 110; 1884.

Eanx thermales, au nord du Chott El-Djerid (Duveyrier); environs de Nefla (Let.).

M. Cossoni Bourguignat, Hisl. Mélan. sysl. europ. p. 111, 1884.

Canaux d'ärrosement au nord du Chott El-Djerid (Duveyrier); Nefta (Let.).

A El-Hammam, près Tozer (Doûm.-Ad.), on rencontre une variété costulée.

M. Dufouri [pars] Ferussac (1), Monogr. Mélan. in Ann. Soc. hist. nat. Paris, I, ${ }_{1}{ }_{2}{ }_{2} 3$, p. 153; Graëlls, Moll. Esp. p. 17, f. 20-22, 1846, Servain, Moll. Esp. p. 153, 1880 , et Bourg. Hist. Mélan. syst. europ. p. $112,1884$.

Quelques échantillons de cette espèce espagnole ont été recueillis aux environs de Tozer (Doûm.-Ad.).

\section{$\$ 7$.}

M. tunetana Morlet, Coq. rec. dans les Chotls par M. André, in Arch. miss. sc. Minist. Inst. publ. ( $3^{e}$ série), VII, 1881 , p. $39^{6}$, pl. VI, f. 3 senlement et in Journ. conch. 188 i, p. 346 , pl. XII, f. 3.

Cette Mélanopside, que nous plaçons en ce moment au rang spécifique, bien que nous l'ayons en 1884 considérée comme une variété de la Mel. Letourneuxi ${ }^{(2)}$, est une forme très répandue dans le sud de la Tunisie, forme qui, malgré ses nombreuses variations de taille et d'ornémentation, conserve néanmoins un cachet caractéristique tout spécial.

Deux séries de formes existent en Tunisie : une costulée, une lisse, et dans chacune de ces séries deux variétés : une minor et une mimima.

(1) Cette synonymie n'est citée seulement que pour le nom.

(2) Bourp. Hist. Nélan. syst. curop. p. 116, 1884 . 
La forme costulée vit aux environs de Keriz, où elle a été recueillie par MIM. André, Duveyrier et Doủmet-Adanson, et ses variétés minor et minima à Tozer.

La forme lisse, ainsi que ses variétés de taille, se rencontre dans les mêmes localités.

s 8.

M. vespertina Bourguignat, Hist. Mélan. syst. europ. p. 194, 1884 .

Cette belle espèce, des frontières marocaines, a été retrouvée dans le Nefzaoua, à Mansoura et à El-Goléa (Let.).

\section{Duveyrierl Bourguignat, sp. nov.}

Testa ovato-ventrosa, crassula, opaca, nitida, atro-castanea ac eleganter striatula; spira breviter attenuata, obtusa (apex sæpissime erosus); anfractibus 5 subconvexiusculis, sat rapide crescentibus, sutura lineari separatis; ultimo permaximo, dimidiam altitudinis superante, convexo, ad aperturam leviter subconcaviusculo, ad insertionem rapide descendente; apertura subobliqua, pyriformi, leviter excentrica, superne acute angulata, inferne in directionem leviter declivem a sinistra ad dextram dilatata, inlus nitida ac vinoso-castanea; columella brevi, arcuatula ac subcontorta, abrupte truncata (sinus truncaturæ profundus); peristomate recto, acuto; margine externo vix subsinuoso ac leviter retrocedente; marginibus callo valido, ad insertionem tuberculoso, junctis; alt. 13 , diam. 7 , alt. ap. 8 millim.

El-Hammam, près Tozer (Dureyrier).

\section{9.}

M. Sevillensis Grateloup, Mém. esp. coq. nouv. (ext. Soc. linn. Bordeaux, 1840, p. 47, pl. IV, f. 10 seulement), et Bourg. Hist. Mélan. syst. europ. p. 152, 1884 .

Cette espèce, qui n'était connue jusqu’à présent que des environs de Séville, a été rencontrée bien caractérisée dans quelques canaux d'irrigation de l'oasis de Tozer (Let.).

M. pleuroplagia Bourguignat, Hist. Mélan. syst. curop. p. 155, 1884.

Cette Mélanopside espagnole, qui n'était également connue que de deux cours d'eau entre Séville et Cordoue, vit avec la précédente dans les eaux de l'oasis de Tozer.

Cette espèce est remarquable par ses grosses côtes espacées, très saillantes, lout à fait obliques de droite à gauche el par son ouverture ayant un certain air excentrique par suite de la courbure de l'axe colımellaire, donl la base est portée à droite, et par suite encore de la grande ventrosité du dernier tour, ventrosité qui rend le côté externe apertural très convexe arrondi. 
M. Maresi Bourguignat, Paléont. Alg. p. 105, pl. VI, f. 1-4, 1862 et Malac. Alg. II, p. 265 , pl. XYI, f. 21-24, 1864 .

Environs de Gabès (Doûm.-Ad.), ruisseau de Keriz (Duveyrier).

C'est un échantillon jeune de cetle localité qui a été figuré (pl. VI, f. 4, in Arch. miss. sc. Minist. Instr. publ.) comme une $M$. tunetana par le commandant Morlet.

\section{NERTTIDE. \\ THEODOXIA (1).}

Th. numidica Bourguignat, 1877 (Neritina numidica, Recluz, Not. Nerita et Neritina in Journ. conch. I, p. 149,1850 et Bourg. Malac. Alg. II, p. 269 , pl. XVI, f. $25-28,1864$ ).

Espèce répandue çà et là dans les sources, les canaux d'irrigation de l'oasis de Gabès, notamment dans l'Aïn Zerig et dans l'Oued Gabès, près le pont de Djara (Let.).

Th. Iuviatilis Locard, Prodr. malac. France, p. $251,{ }_{1882}$ (Nerita fluviatilis, Linnæus, Syst. nat. [éd. x, ${ }_{1758}$ ], p. 777. Neritina fluviatilis, Lamarck, Anim. s. vert. VI $\left[2^{\circ}\right.$ part.], p. 188,1822 . Theodoxus fluviatilis, Issel, Moll. prov. Pise, p. 33,1866$)$.

Le type à coquille mouchetée et zébrée est peu commun, mais c'est surtout la var. nigra (Bourg. Malac. Alg. II, p. 268) que l'on rencontre en grande abondance dans l'Oued Gabès, ainsi que dans presque toutes les eaux de l'oasis de Gabès (Let.).

Le commandant Morlet (Coq. rec. pendant l'explor. des Chotts, in Arch. miss. sc. [ $3^{\mathrm{e}}$ série, VII, 1881], p. 397) signale cette espèce de l'Ain Oudref et de l'Oued Melah (André).

Th. Baetica Locard, Prodr. malac. France, p. 252, 1882 (Neritina Bætica, Lamarck, Anim. s. vert. VI [ $2^{\circ}$ part. $]$, p. 188,1822 ).

Oasis de Gabès, notamment dans l'Aïn Zerig (Let.).

\section{SMARAGDIA (2).}

Sm. viridis Issel, Malac. mar. Rosso, p. 213, 1869 (Nerita viridis, Linnceus, Syst. nat. [éd. xill, 1789], p.1254; .Neritina viridis, Lamarck, 1823; Gaillardotia viridis, Bourg. 1877 et Locard 1882).

Lagunes entre Utique et Porto-Farina, ainsi, que dans les alluvions, près de l'embouchure de la Medjerda (Pechaud et Berthier).

(I) Denys de Montfort, 1810 :

(2) Issel, 1869 .

Malacologie. 


\section{MOLLUSGA AGEPHALA.}

\section{LA ME LLIBRA NCHIATA.}

\section{SPILAEIDAE.}

\section{PISIDIUM.}

P. pusillum Jenyns, Mon. Cycl. Pisid. in Trans. Camb. phil. Soc. IV, p. 302, pl. XX, f. 4-6, 1832 (Tellina pusilla, Gmelin, Syst. nat. I [v1 pars], p. 3231, 1789).

Dans l'Aïn Bizeronin, chez les Sidi-Ali-ben-Amor (Let.).

\section{UNIONI I I A.}

UNIO.

La Régence de Tunis est très pauvre en Mollusques Acépliales. Longtemps on a cru qu'il n'y avait dans ce pays ni Unio ni Anodonta. Les dernières explorations nous permettent néanmoins de signaler 5 espèces d'Unio; quant aux Anodonta, nous pensons quil doit en exister dans les petits lacs du nord, notamment dans ceux au sud de Bizerte. lacs qui malheureusement n'ont pu encore être explorés.

\section{U. Delevieleusso Hagenmüller, spec. nov.}

Concha inæquilaterali, irregulariter subrotundato-oblonga, mediocriter inflata, crassa, ponderosa, parum nitente, valide præsertim ad peripheriam concen/rice striata, atro-castanea aut subolivacea; intus albo-margaritacea; valvis crassis, ponderosis, vix antice posticeque hiantibus, relative mediocriter convexis (convexitas [maximum ad 11 millim. a linea verticali, ad 25 a natibus, ad 55 a rostro, ad $44 \mathrm{ab}$ anteriore margine, ad 32 ab angulo et ad 35 a verticali] super regionem anticam attenuata, in sulco dorsali convexior, nihilominus sat normalis); supra a natibus ad rostrum in orbem bene arcuatum descendente; antice exacte amplo-rotundata; infra leviter convexa, in medio subsinuosa; postice (regio postica duplum anterioris leviter superans) in rostrum inferum retusumque ac quasi leviter subrecurvatum attenuata; umbonibus rotundatis, subprominentibus, potius subcompressis (nates decorticatæ); sulco dorsali arcuatulo, obsoleto; cardine crasso, robusto, arcuato; dente cardinali robusto, producto-trigonali, ad summum crenulato; lamella laterali arcuata, obtuse parum producta et relative valde elongata; lunella exigua, virguliformi; - long. max. 97 ; alt. max. et alt. vertic. æque 54 ; crassit. max. 35 ; bas. apico-rost. 77 ; intervall. a natibus ad angulum 47 , ab angulo ad rostrum 37 et a rostro ad verticalem ac a basi vert. ad angulum æqualiter 57 ; reg. antica 32 , postica 66 millim. 
Cette belle et grande espèce, que le $D^{r}$ Hagenmüller a dédiée à sa mère (née Délevieleuse), a été recueillie par ce malacologiste sur les bords de la Medjerda, non loin de la station de Béja.

\section{U. Doumeti Bourguignat, sp. nov.}

Concha inæquilaterali, transverse oblonga, fere æqualiter antice ac postice rotundata, mediocriter convexa, sat crassa et ponderosa, bene concentrice striata, subolivaceo-castanea, intus candido-margaritacea; valvis crassulis et ponderosis, antice posticeque anguste hiantibus, mediocriter convexis (convexitas [maxim. ad 16 millim. a linea verticali, ad 25 a natibus, ad 43 a rostro, ad 42 ab ant. margine, ad 27 ab angulo et ad 29 a linea verticali] fere regularis); supra a natibus ad angulum, externe vix perspicuum, subrecte arcuatula, dein usque ad rostrum rotundata; antice rotundata, nihilominus parum ampla; infra subconvexa, in medio rectiuscula; postice (regio postica duplum anterioris superans) in rostrum rotundatum, in directionem transversalem, late attenuata; umbonibus compressis, obtuse rotundatis (nates acutæ, leviter desectæ et eleganter striatæ); sulco dorsali male definito, nihilominus superne zonula saturatiore delimitato; cardine arcuato; dente cardinali compresso, alte triangulari ; lamella laterali producta, valde elongata ac ad extremitatem suam abrupte truncata; lunella triangulari; - long. max. 83 ; alt. max. et alt. vert. æque 42 , crassit. max. 27 ; bas. apico-rost. 66 ; intervall. a natibus ad angulum 43 , ab angulo ad rostrum 29 , a rostro ad verticalem 55 , et a basi verl. ad angulum 52 ; reg. antica 26 , postica 59 millim.

La Medjerda, près de Tebourba (Doûm.-Ad.).

\section{Zenaticus, Letourneux, sp. nov.}

Concha inæquilaterali, subrotundalo-ovata, sat compressa, crassula, nitente, eleganter striatula (striæ ad peripheriam foliaceæ), antice luteo-nigrescente, in medio luteola, postice viridi cum zonulis radiantibus, ad umbones tandem pallide cinerescente; intus splendide albomargaritacea; valvis mediocriter crassis, vix hiantibus, parum convexis (convexitas [maxim. ad 27 millim. a linea verticali, ad 19 a natibus, ad 37 a rostro, ad 33 ab ant. margine, ad 24 æque ab angulo et verticali] regulariter ad peripheriam attenuata, nihilominus super umbones quasi compressa); supra arcuatula usque ad angulum, dein rotundata usque ad rostrum; antice late rotundata; infra vix arcuatula aut fere rectiuscula; postice (regio postica mediocris, duplum anterioris non attingens) in rostrum subinferum rotundatumque attenuata; umbonibus compressis, leviter rugosis; sulco dorsali male definito; dente cardinali compresso, alte 
triangulari, ad summum serratulo; lamella laterali subarcuata, producta, stricta, cultrata ac fimbriata; lunella elongato-virguliformi; - long. max. 70 ; alt. max. et alt. vert. æque 40 ; crass. max. 23 ; bas. apicorost. 59 ; intervall. a natibus ad angulum 33 , ab angulo ad rostrum 25 , a rostro ad rerticalem 41 et a basi vertic. ad angulum 43 ; reg. antica 25 , postica 45 millim.

Cet Unio; abondant en Algérie, dans l'Oued Zenati, près Medjez-Ahmar et dans la Seybouse, près Guelma, vit également dans la Nedjerda, où il a été recueilli entre Medjez-el-Bab et Tebourba (Pech.).

U. Durieul Deshayes, Moll. all. Alg. pl. CIX, f. 5-6 et Bourg. Malac. Alg. Il, 1864, 'p. 288 , pl. XIX, f. 1-4 (Unio Sitifiensis Morelet, in Journ. conch. p. 360, 1851).

Celle espèce, répandue daus la province de Constantine, a été constatée dans l'Oued Zergua et la Medjerda, près Tebourba (Hagenm.).

Dans l'Oued Milliana, petite rivière à l'est de Tunis, sur la route d'Hammanel-Lif, se trouve en assez grande abondance une forme minor de cette espèce (Hagenm., Marès).

\section{U. Rouirel Bourguignat, sp. nov.}

Concha parum inæquilaterali (nates sat medianæ), breviter oblonga, supra infraque subparallela, antice posticeque æqualiter rotundata, parum crassula ac ponderosa, nitida, argute striata, pallide castanea, ad regionem anteriorem saturatiore et ad umbones decorticala; intus candida; valvis biliantibus (hiantia anterior aperta, hiantia posterior angusta), parum convexis (convexitas [maxim. ad 9 mill. a linea verticali, ad 16 a natibus, ad 24 a rostro, ad 29 a marg. anteriore, ad 16 ab angulo et ad 19 a verticali] ad peripheriam regulariter attenuata); supra infraque vix subarcuatula; antice late rotundata; postice (regio postica sesquilongitudinem anterioris leviter superans) xque late rotundala et in rostrum perobtusum attenuata; umbonibus compressis, parum prominentibus (nates eleganter nodoso-sulcatæ); sulco dorsali superne zonula saturatiore delimitato; dente cardinali compresso, obtuse triangulari ac ad summum subliliter fimbrialo; lamella laterali producta, compressa, ad extremitatem suam abrupte terminata; ligamento brevi, valido, prominente; lunella elongato-triangulari; - long. max. 58 ; alt. max. et alt. vert. æque 29 ; crassit. max. 18 ; bas. apico-rost. 40 ; intervall. a natibus ad angulum 26 , ab angulo ad rostrum 18 , a rostro ad verticalem $3 a$ et a basi verticalis ad angulum 32 ; reg. antica 20 , postica 32 millim.

Cette espèce, dédiée à M. le médecin militaire Rouire, qui en a fait la découverte, habite avec la précédente, dans l'Oued-Milliana. 


\section{RÊSUMÉ.}

Le premier Mollusque spécial à la Tunisie date de 1850 ; quelques années après, 13 à 14 autres furent découverts par un pasteur anglican et le célèbre voyageur Henri Duveyrier.

14 à 15 espèces existaient donc en 1868 , lorsque parut l'Histoire malacologique de la Régence de Tunis (in- $4^{\circ}$ av. 1 pl.) publiée par M. J.-R. Bourguignat, ouvrage qui porta ce nombre à 61 .

Depuis cette époque, les différentes explorations des savants ou des voyageurs (Bellucci, Kérim, Gestro, Issel, G. Doria, Berthier, André, Pechaud; etc.) ont augmenté ce nombre à peine d'une vingtaine.

La faune malacologique formait donc, avant l'arrivée des membres de la Mission, un ensemble de 75 à 80 espèces.

Actuellement cette faune atteint le chiffre énorme de 473 espèces, compris dans 37 genres.

Sur ces 473 espèces, nous en présentons 256 comme nouvelles.

Ces especes sont ainsi réparties :

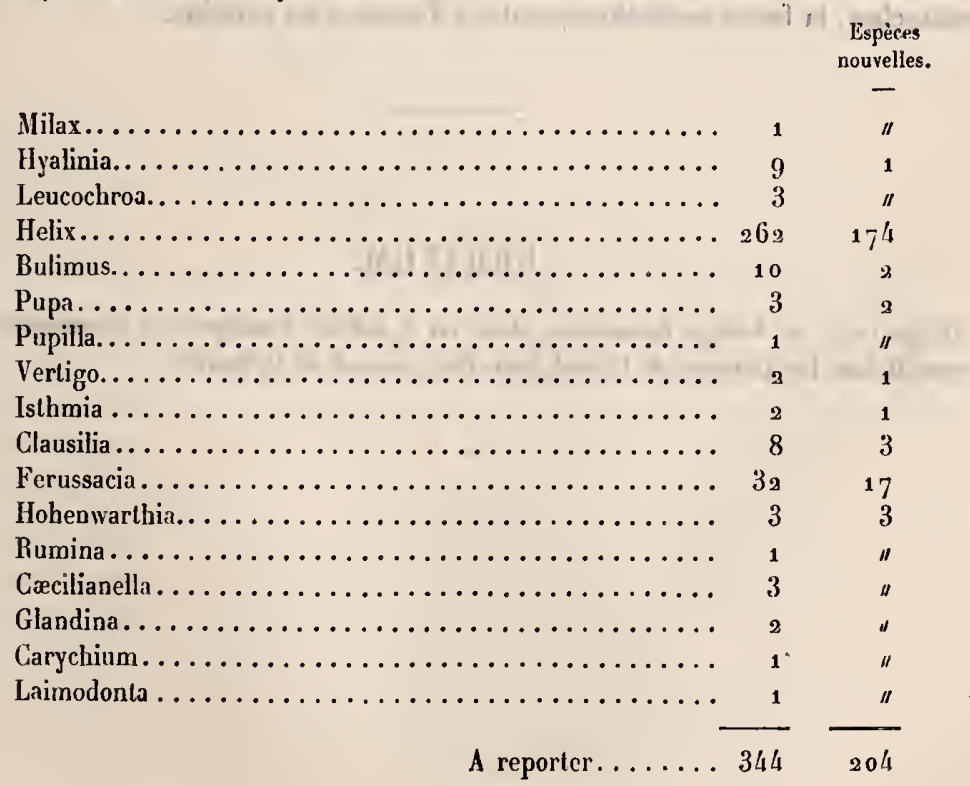




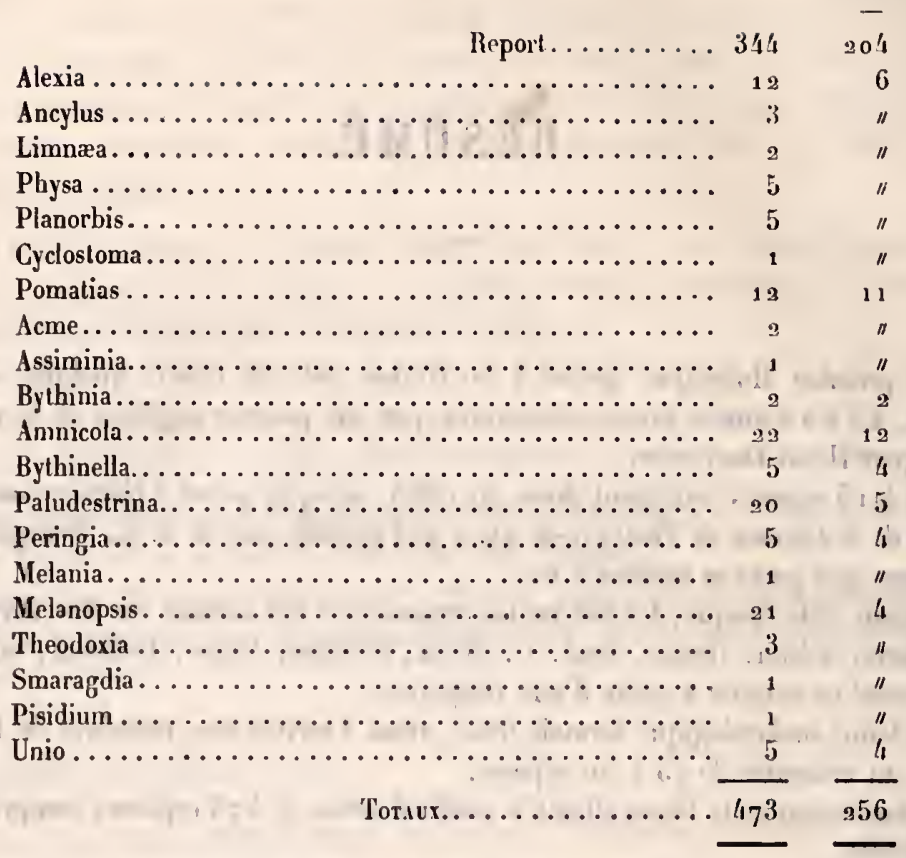

On voit donc, par ce tableau, combien, grâce à nos investigations et à nos recherches, la faune malacologique de la Tunisie s'est enrichie.

\section{ERRATUM.}

Page 109: le Vertigo Latasteana, dont on a oublié d'indiquer la provenance, a été recueilli dans les alluvions de l'Oued Sidi-Aïch, au sud de la Tunisie. 

